\title{
Level Densities and Radiative Strength Functions in ${ }^{56} \mathrm{Fe}$ and ${ }^{57} \mathrm{Fe}$
}

\author{
E. Tavukcu
}

(Ph. D Thesis)

December 10, 2002

U.S. Department of Energy

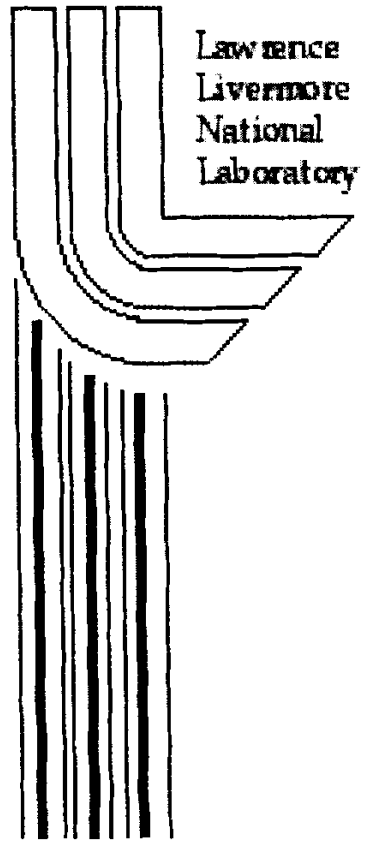




\section{DISCLAIMER}

This document was prepared as an account of work sponsored by an agency of the United States Government. Neither the United States Government nor the University of California nor any of their employees, makes any warranty, express or implied, or assumes any legal liability or responsibility for the accuracy, completeness, or usefulness of any information, apparatus, product, or process disclosed, or represents that its use would not infringe privately owned rights. Reference herein to any specific commercial product, process, or service by trade name, trademark, manufacturer, or otherwise, does not necessarily constitute or imply its endorsement, recommendation, or favoring by the United States Government or the University of California. The views and opinions of authors expressed herein do not necessarily state or reflect those of the United States Government or the University of California, and shall not be used for advertising or product endorsement purposes.

This work was performed under the auspices of the U. S. Department of Energy by the University of California, Lawrence Livermore National Laboratory under Contract No. W-7405-Eng-48.

This report has been reproduced directly from the best available copy.

Available electronically at http://www.doe.gov/bridge

Available for a processing fee to U.S. Department of Energy and its contractors in paper from

U.S. Department of Energy

Office of Scientific and Technical Information P.O. Box 62

Oak Ridge, TN 37831-0062

Telephone: (865) 576-8401

Facsimile: (865) 576-5728

E-mail: reports@adonis.osti.gov

Available for the sale to the public from

U.S. Department of Commerce

National Technical Information Service 5285 Port Royal Road

Springfield, VA 22161

Telephone: (800) 553-6847

Facsimile: (703) 605-6900

E-mail: orders@ntis.fedworld.gov

Online ordering: http://www.ntis.gov/ordering.htm

OR

Lawrence Livermore National Laboratory

Technical Information Department's Digital Library

http://www.llnl.gov/tid/Library.html 
LEVEL DENSITIES AND

RADIATIVE STRENGTH FUNCTIONS IN ${ }^{56} \mathrm{FE}$ AND ${ }^{57} \mathrm{FE}$

by

EMEL TAVUKCU

A dissertation submitted to the Graduate Faculty of

North Carolina State University

in partial fulfillment of the

requirements for the Degree of

Doctor of Philosophy

Department of Physics

Raleigh, North Carolina

2002

APPROVED BY:

Gary E. Mitchell,

John F. Shriner, Jr.

Chair of Advisory Committee

D. Ronald Tilley

Diane Markoff 


\begin{abstract}
TAVUKCU, EMEL. Level Densities and Radiative Strength Functions in ${ }^{56} \mathrm{Fe}$ and ${ }^{57} \mathrm{Fe}$. (Under the direction of Prof. Gary E. Mitchell.)

Understanding nuclear level densities and radiative strength functions is important for pure and applied nuclear physics. Recently, the Oslo Cyclotron Group has developed an experimental method to extract level densities and radiative strength functions simultaneously from the primary $\gamma$ rays after a light-ion reaction. A primary $\gamma$-ray spectrum represents the $\gamma$-decay probability distribution. The Oslo method is based on the AxelBrink hypothesis, according to which the primary $\gamma$-ray spectrum is proportional to the product of the level density at the final energy and the radiative strength function. The level density and the radiative strength function are fit to the experimental primary $\gamma$-ray spectra, and then normalized to known data. The method works well for heavy nuclei. The present measurements extend the Oslo method to the lighter mass nuclei ${ }^{56} \mathrm{Fe}$ and ${ }^{57} \mathrm{Fe}$. The experimental level densities in ${ }^{56} \mathrm{Fe}$ and ${ }^{57} \mathrm{Fe}$ reveal step structure. This step structure is a signature for nucleon pair breaking. The predicted pairing gap parameter is in good agreement with the step corresponding to the first pair breaking. Thermodynamic quantities for ${ }^{56} \mathrm{Fe}$ and ${ }^{57} \mathrm{Fe}$ are derived within the microcanonical and canonical ensembles using the experimental level densities. Energy-temperature relations are considered using caloric curves and probability density functions. The differences between the thermodynamics of small and large systems are emphasized. The experimental heat capacities are compared with the recent theoretical calculations obtained in the Shell Model Monte Carlo method. Radiative strength functions in ${ }^{56} \mathrm{Fe}$ and ${ }^{57} \mathrm{Fe}$ have surprisingly high values at low $\gamma$-ray energies. This behavior has not been observed for heavy nuclei, but has been observed in other light- and medium-mass nuclei. The origin of this low $\gamma$-ray energy effect remains unknown.
\end{abstract} of California, Lawrence Livermore National Laboratory under Contract No. W-7405-Eng-48. 


\section{Biography}

Emel Tavukçu

Personal:

Born 18 April 1972, Antalya, Turkey

Education:

B.S. in Physics, Ege University, 1993

M.S. in Physics, Western Michigan University, 1997

Academic Positions:

Teaching Assistant, Osmangazi University, 1995-1995

Fellowship from Higher Education Council of Turkey (YÖK), 1995-1999

Research Assistant, North Carolina State University, 1999-2002 


\section{Acknowledgments}

I am deeply thankful to my thesis advisor Gary Mitchell for being an excellent supervisor, for his support and encouragement, and for giving me the opportunity to work at several laboratories nationwide and abroad. Although I regret working away from him, I feel fortunate to work with other great physicists in this field. I am grateful to John Becker and Lee Bernstein for their involvement in my research, for their support and warm hospitality during my stay at Livermore.

I am deeply thankful to three people from whom I have acquired deep knowledge of nuclear physics: I thank Paul Garrett for his infinite patience and his ability to teach with simple pictures. I have learned much from him. I thank Walid Younes for always finding time to answer my questions at the cost of delaying his own work, and for many fruitful discussions. I shall regret not having him next door to consult. I thank Andreas Schiller for helping me with the data analysis, for many illuminating discussions, and for his careful reading of this manuscript. I truly appreciate his contribution to this work.

For the experiment performed at the Oslo Cyclotron Laboratory I am grateful to Eivind Olsen and Jon Wikne for providing excellent experimental conditions, to Magne Guttormsen, Finn Ingebretsen, Elin Melby, Stein Ødegård, John Rekstad, Sayed Rezazadeh, and Sunniva Siem for helping with taking shifts. Their exceptional hospitality is greatly appreciated. I especially thank Magne Guttormsen for explaining several aspects of the data sorting codes and for stimulating discussions. I wish to thank Alexander Voinov of Dubna for his friendly collaboration.

Thanks to the colleagues in N Division at the Lawrence Livermore National Laboratory for a friendly working atmosphere. I thank John Shriner, Ronald Tilley, Diane Markoff, and David Gilmartin for kindly agreeing to serve on my thesis committee. I thank Undraa Agvaanluvsan and Mine Ilk for their friendship and trust.

Finally, I thank my dear parents, Rüstem and Fevziye Tavukçu, my sisters Serap, Sibel, and Nalân, and my brother Mehmet for their great love, support, and high expectations. 


\section{CONTENTS}

LIST OF TABLES $\ldots \ldots \ldots \ldots \ldots \ldots \ldots \ldots \ldots \ldots$ vii

LIST OF FIGURES $\ldots \ldots \ldots \ldots \ldots \ldots \ldots \ldots \ldots$ viii

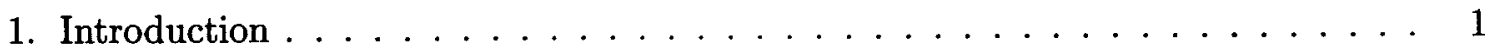

2. Level Densities, Thermodynamics, Radiative Strength Functions _... . . . 4

2.1 Level Densities . . . . . . . . . . . . . . . . . 4

2.2 Thermodynamics, Statistical Mechanics $\ldots \ldots \ldots \ldots \ldots \ldots \ldots$

2.2 .1 Microcanonical Ensemble $\ldots \ldots \ldots \ldots \ldots \ldots$

2.2 .2 Canonical Ensemble $\ldots \ldots \ldots \ldots \ldots \ldots \ldots$

2.3 Radiative Strength Functions . . . . . . . . . . . . . 14

2.3.1 E1 Strength Function $\ldots \ldots \ldots \ldots \ldots \ldots \ldots \ldots$

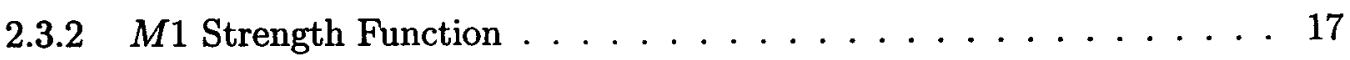

3. Method to Extract Level Density and Radiative Strength Function Simultaneously 19

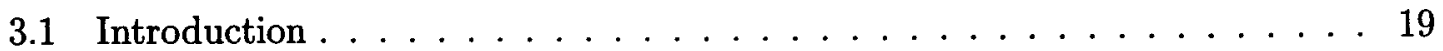

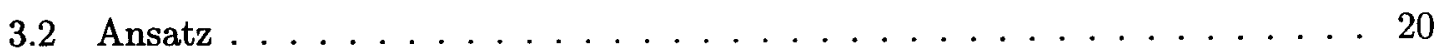

3.2.1 Axel-Brink Hypothesis . . . . . . . . . . . . . . 20

3.2.2 Normalization of Eq. 3.2 at each $E_{x} \ldots \ldots \ldots \ldots \ldots$

3.2.3 Other Solutions of the Primary $\gamma$-ray Matrix . . . . . . . 24

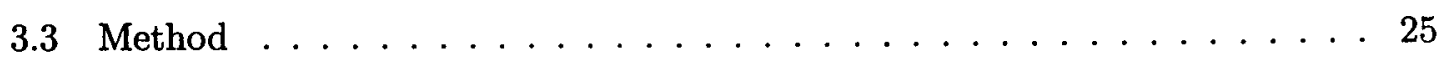

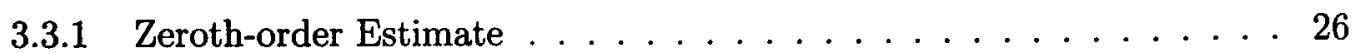

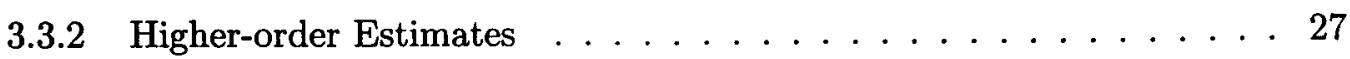

3.4 Convergence Properties $\ldots \ldots \ldots \ldots \ldots \ldots \ldots$

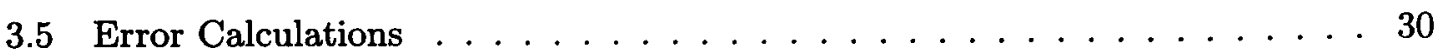

3.6 Normalization of the Level Density to Experimental Data . . . . . . . . 32 
3.7 Testing the Method on Theoretical Spectra . . . . . . . . . . . . 34

3.8 Application of the Method to ${ }^{56} \mathrm{Fe}$ and ${ }^{57} \mathrm{Fe}$ Spectra . . . . . . . . . 36

4. Experimental Details . . . . . . . . . . . . . . . . . 39

4.1 Introduction . . . . . . . . . . . . . . . . 39

4.2 CACTUS Multidetector Array . . . . . . . . . . . . . 40

4.2 .1 Silicon Particle Detectors . . . . . . . . . . . . . . 42

4.2 .2 Gamma-ray Detectors . . . . . . . . . . . . . 4 45

4.3 Electronics . . . . . . . . . . . . . . . . . 47

5. Data Analysis . . . . . . . . . . . . . . . . . . . 52

5.1 The ${ }^{57} \mathrm{Fe}\left({ }^{3} \mathrm{He}, \alpha \gamma\right){ }^{56} \mathrm{Fe}$ Reaction Mechanism . . . . . . . . . . . . 52

5.2 Particle-Spectra Analysis . . . . . . . . . . . . . . 54

5.3 Data Reduction . . . . . . . . . . . . . . . . 56

5.4 Gating on $\alpha$ Particles $\ldots \ldots \ldots \ldots \ldots 7$

5.5 Particle $-\gamma$-ray Coincidence Timing . . . . . . . . . . 61

$5.6 \mathrm{NaI}(\mathrm{Tl})$-spectra Analysis . . . . . . . . . . . . . . . . . . 64

5.7 Particle $-\gamma$-ray Matrix . . . . . . . . . . . . . 66

5.8 Unfolding the Total $\gamma$-ray Spectra . . . . . . . . . . . . 68

5.8.1 Response Function . . . . . . . . . . . . . . . . 70

5.8.2 The Folding Iteration Method . . . . . . . . . . . . . . . 74

5.8.3 The Compton Subtraction Method . . . . . . . . . . . 76

5.9 Extracting Primary $\gamma$-ray Spectra . . . . . . . . . . . . 78

6. Experimental Results on ${ }^{56} \mathrm{Fe}$ and ${ }^{57} \mathrm{Fe} \ldots \ldots \ldots$

6.1 Level Densities . . . . . . . . . . . . . . . . 86

6.2 Radiative Strength Functions . . . . . . . . . . . . . . 92

6.3 Level Densities and $\gamma$-ray Strength Functions for Rare-Earth Nuclei . . . 100

6.4 Thermodynamic Properties . . . . . . . . . . . . . . . 103

6.4.1 Caloric Curves in Microcanonical and Canonical Ensembles . . . . . 104

6.4.2 Probability Density Function . . . . . . . . . . . . . . . 109

6.4 .3 Heat Capacity . . . . . . . . . . . . . . . . . 110

7. Summary and Outlook . . . . . . . . . . . . . . . . 115

7.1 Summary and Conclusions . . . . . . . . . . . . . . . 115

7.2 Outlook .............................. 118

A. Transformation Functions $\ldots \ldots \ldots \ldots \ldots \ldots$ 


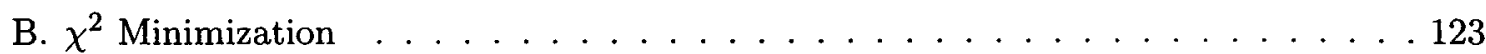

C. Area Correction for the Primary $\gamma$-ray Spectrum . . . . . . . . . . . 130

D. Caloric Curves Derived from Probability Density Function . . . . . . . . . . 132

REFERENCES . . . . . . . . . . . . . . . . . . . 140 


\section{LIST OF TABLES}

3.1 Percentage $p$ as a function of the number of iterations. . . . . . . . 30

4.1 Impurities in the ${ }^{57} \mathrm{Fe}$ target. . . . . . . . . . . . . . . 39

4.2 Beam types and their maximum energies produced at the OCL. . . . . . . 40

4.3 Position of the $\mathrm{NaI}(\mathrm{Tl})$ detectors. . . . . . . . . . . . . . . . 41

4.4 Position of the $60 \%$ HPGe detector. . . . . . . . . . . . . . . 42

4.5 Thicknesses and leakage currents for the end detectors. . . . . . . . . . . . 43

4.6 Flags or hit patterns for the TPUs. . . . . . . . . . . . . . 51

4.7 Event matrix generated by an offline program. . . . . . . . . . . . . . 51

5.1 Intensities of the $\mathrm{NaI}(\mathrm{Tl})$ response functions. . . . . . . . . . . 72

$6.1 \quad$ Pairing gap parameters. . . . . . . . . . . . . . 91 


\section{LIST OF FIGURES}

$3.1 \quad$ Normalized experimental primary $\gamma$-ray matrix for ${ }^{56} \mathrm{Fe} . \ldots \ldots 21$

3.2 Statistical decay in the continuum energy region. . . . . . . . . . . . 22

3.3 Schematic illustration of primary $\gamma$-ray matrix. . . . . . . . . . 23

3.4 Schematic illustration of performing the sum in Eqs. 3.16 and 3.17. . . . . . 29

3.5 Test of the extraction method. . . . . . . . . . . . . . 35

$3.6 \quad$ Experimental and calculated primary $\gamma$-ray spectra in ${ }^{56} \mathrm{Fe} . \ldots \ldots 37$

3.7 Experimental and calculated primary $\gamma$-ray spectra in ${ }^{57} \mathrm{Fe} . \ldots . \ldots 38$

4.1 Schematic drawing of the CACTUS multidetector array. . . . . . . . . . 41

4.2 Schematic drawing of a Si particle detector. . . . . . . . . . . . 43

4.3 Schematic drawing of a Ge detector. . . . . . . . . . . . 45

4.4 Schematic drawing of a $\mathrm{NaI}(\mathrm{Tl})$ detector. . . . . . . . . . . . 46

4.5 Diagram of electronics in the experimental room. . . . . . . . . . 48

4.6 Diagram of electronics in the control room. . . . . . . . . . . . . . . 50

5.1 Particle spectra for the front and end particle detectors. . . . . . . . . 55

5.2 Total particle energy spectrum. . . . . . . . . . . . . . 57

$5.3 \triangle \mathrm{E}$ vs. E spectrum obtained from telescope $7 \ldots \ldots \ldots \ldots$

5.4 Range curve for $\alpha$ particles in silicon. . . . . . . . . . . . . 60

5.5 Thickness spectrum of particle telescope $7 \ldots \ldots \ldots 1$

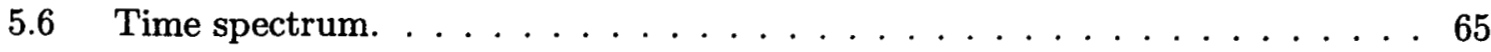


5.7 Energy calibration for the $\mathrm{NaI}(\mathrm{Tl})$-detectors. . . . . . . . . . . 66

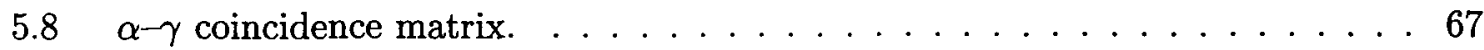

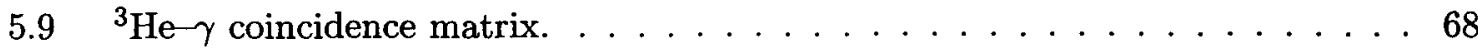

5.10 Particle spectra gated on $\gamma$ rays. . . . . . . . . . . . . 69

5.11 Interpolation of Compton background. . . . . . . . . . 73

5.12 Schematic representation of the subtraction method . . . . . . . . . 79

$5.13 \gamma$ rays depopulating the level $\mathrm{E}_{i} \ldots \ldots \ldots \ldots \ldots$

5.14 Determination of the weighting function. . . . . . . . . 82

5.15 Raw, unfolded, and primary $\gamma$-ray spectra. . . . . . . . . 85

6.1 Normalization of the ${ }^{57} \mathrm{Fe}$ level density. . . . . . . . . . . . 88

6.2 Normalization of the ${ }^{56} \mathrm{Fe}$ level density. . . . . . . . . . . . . . . . . 90

6.3 Level densities for ${ }^{56} \mathrm{Fe}$ and ${ }^{57} \mathrm{Fe} \ldots \ldots \ldots \ldots \ldots$

$6.4 \gamma$-ray transmission coefficient for ${ }^{56} \mathrm{Fe} \ldots \ldots \ldots \ldots$

$6.5 \gamma$-ray transmission coefficient for ${ }^{57} \mathrm{Fe} \ldots \ldots \ldots \ldots$

6.6 Radiative strength function for ${ }^{56} \mathrm{Fe} \ldots \ldots \ldots 7$

6.7 Radiative strength function for ${ }^{57} \mathrm{Fe} . \ldots \ldots \ldots$

6.8 Level densities for rare-earth nuclei. . . . . . . . . . . . . . . . . 101

$6.9 \gamma$-ray strength functions for rare-earth nuclei. . . . . . . . . . . 103

6.10 Experimental caloric curves for ${ }^{56} \mathrm{Fe} \ldots \ldots \ldots$

6.11 Experimental caloric curves for ${ }^{57} \mathrm{Fe} \ldots \ldots \ldots$

6.12 Canonical caloric curves for ${ }^{56,57} \mathrm{Fe} \ldots \ldots \ldots$. . . . . . . . . . . . . .

6.13 Probability density function for ${ }^{56} \mathrm{Fe} \ldots \ldots \ldots \ldots$

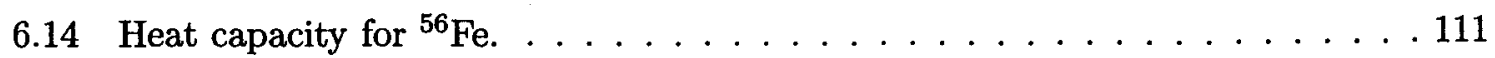

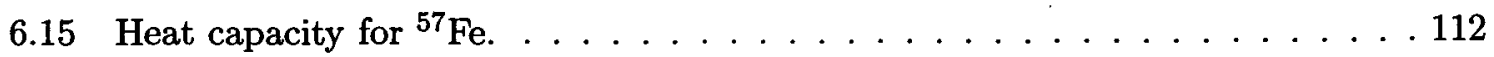

6.16 Sensitivity of heat capacity to the slope of level density. . . . . . . . . . 114

D.1 Probability density function for small systems. . . . . . . . . . 135

D.2 Probability density function for large systems. . . . . . . . . . 136 
D.3 Effect of entropy fluctuations on the caloric curves. . . . . . . . . . . 137

D.4 Probability density function $P(E, T)$ for ${ }^{56} \mathrm{Fe} \ldots \ldots \ldots \ldots$ 


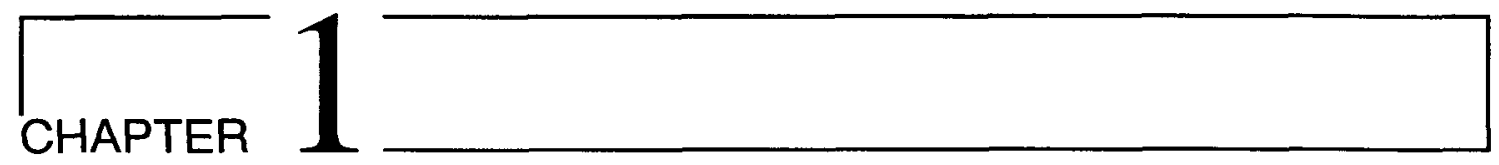

\section{Introduction}

Nuclei are complex quantum mechanical systems. A complete description of nuclear structure is contained in the nuclear wave function. However, it is essentially impossible to derive nuclear wave functions from first principles. This is partly because the knowledge of the strong nuclear interaction is still limited. However, even if the strong nuclear force were completely known, it would still be desirable to develop simplified models containing essential nuclear features. The nuclear shell model and its extension to deformed nuclei, the Nilsson model, are two models used to explain the single-particle behavior of nuclei as well as some "collective" effects. Other models have also been developed to account for collective nuclear behavior.

One important nuclear property is the nuclear level density. Level densities are crucial for the study of nuclear reactions. Applications include astrophysics, the study of nuclei far from the stability line, and stockpile stewardship. The first theoretical attempt to describe nuclear level densities was by Bethe in 1936. In his fundamental work, Bethe described the nucleus as a gas of noninteracting fermions moving freely in equally-spaced single-particle orbits. The level density was determined by calculating the entropy from Fermi statistics. Bethe's formula was later modified in order to account for other nuclear features, such as shell effects and residual interactions. Recently the level density problem has been treated with a variety of modern methods. The shell model Monte Carlo (SMMC) method seems promising in calculating level densities while explicitly predicting parity and 
angular momentum dependence of level densities.

Pairing correlations in odd- $\mathrm{A}$ and in particular in even-even nuclei also affect the level density at low excitation energies. At low temperatures nucleons are paired off in time-reversed orbits. An important consequence of this pairing effect is the energy gap between the ground state and the lowest two-quasiparticle excitation in even-even nuclei. This effect manifests itself in the level density as a rapid increase in the number of levels above this energy gap.

Nuclear level densities are also important to infer nuclear thermodynamic properties. Thermodynamic quantities have been used to investigate phase transition phenomena in nuclei. Heat capacities have been calculated theoretically for several iron isotopes within the SMMC model, and shown to reveal a signature for a pairing "phase transition". An $S$-shaped structure in the heat capacity curve derived from experimental level densities has been interpreted as a transition from a phase with strong pairing correlations to a phase with weaker pairing correlations.

The radiative strength function (RSF) is another important nuclear property. Most experimental information on RSFs has been obtained from photonuclear reactions. Direct observation of giant dipole resonances in photonuclear reactions led to resonance models for the RSF. Within this model the electric field of the photon acts only on protons, and the neutrons and protons move in opposite directions while keeping the center of mass at rest. However, extensive experimental information on RSFs, particularly at low $\gamma$-ray energies, are necessary in order to verify and/or improve these models.

Recently, a method to extract both the level density and the radiative strength function has been developed by the Oslo Cyclotron group. This Oslo method employs the experimental primary $\gamma$-ray spectra from all excitation energies as a starting point, and is based on the Axel-Brink hypothesis. The Oslo method has been shown to work well in the rare-earth nuclei.

Extending the Oslo method to lower mass regions was the primary motivation of this dissertation. Astrophysical interest and the recent theoretical prediction of pairing phase transitions in the iron isotopes led us to study the ${ }^{56} \mathrm{Fe}$ and ${ }^{57} \mathrm{Fe}$ isotopes. 
This thesis consists of seven chapters and four appendices. Historical background on level densities and radiative strength functions, and an introduction to thermodynamic quantities and statistical ensembles are given in the following chapter. In Chapter 3 the method to extract the level density and radiative strength function simultaneously is described, error calculations, and normalization procedures are discussed, and the application of the method to simulated theoretical data and to the present experimental data is presented. Chapter 4 includes experimental details. In the next chapter the analysis of the experimental data is described, including unfolding the total $\gamma$-ray spectra and extracting primary $\gamma$-ray spectra for all excitation-energy bins. Chapter 6 contains experimental results on level densities and radiative strength functions on ${ }^{56} \mathrm{Fe}$ and ${ }^{57} \mathrm{Fe}$. Thermodynamic properties derived from the level densities are presented, and compared with corresponding theoretical calculations. This is followed by a short overview on earlier results on rare-earth nuclei using the Oslo method. Chapter 7 summarizes the conclusions of this work, and provides suggestions for future research. Mathematical details are given in the appendices. In Appendix A transformation functions, which give all solutions of the primary $\gamma$-ray matrix, are derived. Appendix B contains mathematical derivations of the least $\chi^{2}$ method, which provide the formulae to calculate new level density and $\gamma$-ray transmission coefficient estimates in the iterative procedure by minimizing the reduced $\chi^{2}$. Appendix $C$ describes an area correction method, which reduces possible errors in obtaining the primary $\gamma$-ray spectra. In Appendix D caloric curves are derived from the probability density function for simple large and small systems. Some difficulties in applying thermodynamic methods to small systems are emphasized. 
CHAPTER 2

\section{Level Densities, Thermodynamics,}

\section{Radiative Strength Functions}

\subsection{Level Densities}

Besides their fundamental importance in nuclear structure, nuclear level densities are the basic input parameters in nuclear reaction theory. Calculations of neutron- and protoncapture reaction rates in nuclear astrophysics require knowledge of the level densities. There are many other applications of level densities, including accelerator-driven transmutation of nuclear waste and radiochemical analysis.

Experimental level densities are usually obtained from direct counting at low excitation energies $\left(E_{x} \leq 5 \mathrm{MeV}\right)$ [1], from study of neutron and proton resonance data [2], from charged-particle evaporation spectra at intermediate energies ( $5 \leq E_{x} \leq 15 \mathrm{MeV}$ ) $[3,4]$, and from Ericson fluctuation analysis at high excitation energies $\left(E_{x} \geq 15 \mathrm{MeV}\right)$ $[3]$.

Most of these experimental data have been interpreted within the framework of Bethe's pioneering Fermi-gas model [5] which gives the energy-dependent level density $\rho(E)$ as 


$$
\rho(E)=\frac{\exp (2 \sqrt{a E})}{12 \sqrt{2} a^{1 / 4} E^{5 / 4} \sigma}
$$

where $\sigma$ is the spin cutoff parameter, $a$ is the level density parameter given by $a=$ $\frac{\pi^{2}}{6}\left(g_{p}+g_{n}\right)$, and $g_{p}$ and $g_{n}$ are the single-particle level density parameters for protons and neutrons, respectively, which is expected to be proportional to the nuclear mass $A$. In this model, particles are considered to move independently; and single-particle states are assumed to be equally spaced. As a result of these assumptions, the nuclear partition function is written as a product of the individual partition functions of constituent nucleons. Then the level density is obtained by the inverse Laplace transform of the partition function. The Bethe formula predicts an exponential increase in the number of levels with excitation energy and atomic mass. This is qualitatively consistent with the experimental data. However, due to the independent-particle and equidistant-level assumptions, this formula does not take into account, e.g., odd-even and collective effects. Therefore, various phenomenological modifications of this simple formula have been proposed in order to account for these effects. These new approaches introduced some free parameters in order to fit the existing experimental data while approximately keeping the functional form of the energy dependence. In the shifted Fermi-gas model, the odd-even effects are included, i.e., the effective excitation energy is reduced by the conventional pairing energy for the odd-mass and even-even nuclei, resulting in a lower level density for the same excitation energy. The standard form is

$$
\rho(U, J)=f(J) \frac{1}{12 \sigma \sqrt{2}} \frac{\exp 2 \sqrt{a U}}{a^{1 / 4} U^{5 / 4}}
$$

where $U$ is the excitation energy shifted by the pairing energy, and $f(J)$ gives the spin distribution of the levels 


$$
f(J)=\frac{(2 J+1)}{2 \sigma^{2}} \exp \left(\frac{-(J+1 / 2)^{2}}{2 \sigma^{2}}\right)
$$

The so-called spin cutoff parameter $\sigma$ is approximately equal to the mean value of the spin distribution at a given energy, and expressed as

$$
\sigma^{2}=0.0888 \sqrt{a U} A^{2 / 3}
$$

Gilbert and Cameron proposed a composite level-density formula which combines a constant temperature formula (CTF)

$$
\rho(E)=\frac{1}{T} \exp \left[\left(E-E_{0}\right) / T\right]
$$

for low energies, and the shifted Fermi-gas model at higher excitation energies [6]. The free parameters introduced by this model are fitted to experimental data at low and high excitations in order to give better absolute values of the nuclear level densities. Another approach, the back-shifted Fermi-gas model (BSFG), was later proposed in order to account for collective and shell effects. In this model, the level density parameter $a$ and the energy shift are considered as free parameters, which allows a reasonable fit to the experimental level densities over a wider range of excitation energies. Later, other phenomenological methods were developed to describe the thermal-damping of shell effects, i.e., the energy dependence of the parameter $a$ (e.g., $[7,8,9]$ ).

All of these approaches are semiempirical and based on experimental data. Reasonable agreement with the experimental data was obtained by introducing new parameters to the original Bethe formula. However, the dependence of these parameters on mass number, excitation energy, and shell effects makes their determination difficult.

Alternatively, numerical statistical calculations have been performed using the usual 
independent-particle model, and including BCS theory in order to account for pairing effects $[10,11]$. A combinatorial method was also introduced which calculates the level density numerically by counting nuclear excited configurations [12, 13]. However, this method requires long computational time, particularly for high excitation energies and for large shell model spaces. The residual interactions were taken into account in spectral distribution method $[14,15]$. This approach is based on expanding the basis states in terms of the Hamiltonian eigenfunctions; then the corresponding intensity as a function of the Hamiltonian eigenvalues is a spectral distribution. The averaged distribution of the Hamiltonian eigenvalues is calculated by studying the energy moments of this distribution. This method is limited to a low-order expansion, which does not give accurate results for large shell model spaces. As an alternative to the traditional combinatorial method, a Monte Carlo method was proposed by Cerf [16] in order to avoid the exhaustive counting of the excited levels.

Recently, a variety of methods have been developed, which include relativistic mean field theory [17], finite temperature random phase approximation [18], finite temperature Hartree-Fock-Bogoliubov method [19], continuous binomial function method [20], and shell model Monte Carlo method (SMMC) [21, 22, 23].

\subsection{Thermodynamics, Statistical Mechanics}

Phenomenological thermodynamics and statistical mechanics have been effective tools to study macroscopic systems in equilibrium. In thermodynamics, the system is described from a macroscopic point of view by introducing appropriate quantities such as volume, pressure, and temperature. However, since there are a large number of constituent particles in a macroscopic system, in general it is difficult to derive thermodynamic properties of any given system from its corresponding microscopic description (the methods of quantum mechanics or classical mechanics). On the other hand, a large number of particles makes it easy to treat the problem through statistical methods. Thus statistical mechanics aims to describe a specific system at the microscopic level from the laws of molecular dynamics, and to derive or predict the laws of thermodynamics from microscopic 
considerations.

In thermodynamics a macroscopic system is described by equations of state, which give a functional relationship among thermodynamic variables for the system in equilibrium. Although microscopic changes do not appear explicitly at the macroscopic level, their effects are incorporated in a macroscopic quantity entropy $S$

$$
d Q=T d S
$$

where $T$ is the temperature of the system, and $d Q$ is the heat absorbed by the system. The concept of equilibrium is linked closely to the idea of temperature. The definition of equilibrium is the equipartition of the available energy among all degrees of freedom. Therefore, temperature is one of the variables that characterizes systems in equilibrium. A system in equilibrium must satisfy either the entropy-maximum principle (for a given energy) or the energy-minimum principle (for a given entropy). These principles can be rewritten in terms of thermodynamic potentials so that intensive variables, such as temperature, can be treated as independent variables. For example, consider a system of fixed volume in contact with a heat reservoir at a constant temperature $T$. In thermal equilibrium the temperature of the system is equal to the temperature of the surroundings, i.e., $(\partial E / \partial S)_{V, N}=T$ (see Eq. 2.6). Alternatively a potential, the so-called Helmholtz potential or Helmholtz free energy, can be defined such that

$$
F=E-T S \text {, }
$$

from which the equilibrium condition reduces to minimizing the Helmholtz potential: $(\partial F / \partial S)_{V, N}=0$. Using the first law of thermodynamics 


$$
\begin{aligned}
d E & =d Q+d W \\
& =T d S-P d V
\end{aligned}
$$

one obtains

$$
d F=-S d T-P d V
$$

The entropy in the first term of this equation tends to increase disorder in the system, while the second term tends to keep the system in order. The Helmholtz potential is more convenient to use in many practical situations. Thermodynamic functions such as pressure $P$ and entropy $S$ can then be obtained from $F(T, V, N)$ in Eq. 2.9

$$
\begin{aligned}
& P=-(\partial F / \partial V)_{T, N} \\
& S=-(\partial F / \partial T)_{V, N}
\end{aligned}
$$

Another thermodynamic quantity is the heat capacity $C_{V}$, which is a measure of the response of the system to changes in its temperature

$$
C_{V}=\left(\frac{d Q}{d T}\right)_{V, N}
$$

at constant volume and particle number $N$. We can also express heat capacity in terms of the Helmholtz potential using Eqs. 2.6 and 2.10

$$
C_{V}=-T\left(\frac{\partial^{2} F}{\partial T^{2}}\right)_{V, N}
$$


These relations for thermodynamic quantities are used to make the connection between statistical and thermodynamic descriptions of systems in equilibrium.

In order to describe a system from microscopic considerations, the basic rule is the postulate of equal apriori probabilities: if an isolated system is in equilibrium, the system is equally likely to be in any of the accessible states. If $\Omega(E, N)$ represents the number of states of the system with fixed volume $V$ and particle number $N$ that lie in the vicinity $(\Delta E)$ of energy $E$, then the probability $P_{i}$ of the system to be in a specific microstate $i$ will be $1 / \Omega$. The term 'probability' is, in general, used to indicate recurrence of an event when measurements are repeated a large number of times. In order to use this interpretation, the concept 'ensemble' is introduced in statistical mechanics. An ensemble is a large number of identical systems whose macrostates are the same, and whose microstates are distributed with equal apriori probability.

There are three commonly-used ensembles in statistical mechanics: Microcanonical, canonical, and grandcanonical ensembles. All three ensembles give the same result in describing systems in the thermodynamic limit. Here, microcanonical and canonical ensembles are relevant, and will be discussed at some length.

\subsubsection{Microcanonical Ensemble}

A microcanonical ensemble consists of isolated physical systems with fixed particle number $N$, and with energy $E$ within a small uncertainty $\Delta E \ll E$. The microstates of the systems are distributed according to the postulate of equal apriori probability. The number of microstates $\Omega(E, N)$ is proportional to the density of states such that

$$
\Omega(E, N)=\rho(E, N) \times \Delta E
$$

It is also convenient to introduce the microcanonical partition function, the entropy of the system, which makes the connection between the microcanonical ensemble and thermodynamics, by 


$$
\begin{aligned}
S(E, N) & =k_{B} \ln \Omega(E, N) \\
& =k_{B} \ln \rho(E, N)+k_{B} \ln \Delta E
\end{aligned}
$$

where $k_{B}$ is Boltzmann's constant. All of the thermodynamics of a system can be obtained from Eq. 2.14. For example, temperature in the microcanonical ensemble is defined as

$$
\frac{1}{T}=\frac{\partial S(E, N)}{\partial E}
$$

This equation gives the relation between the temperature and energy of the system, and is called the caloric curve in the microcanonical ensemble.

\subsubsection{Canonical Ensemble}

When a physical system is in thermal equilibrium with a heat reservoir at constant temperature $T$ and can exchange energy (but not particles) with the reservoir, the ensemble of such systems is called a canonical ensemble. Analogous to the entropy in the microcanonical ensemble, the partition function is the starting point to obtain the thermodynamics of a system in the canonical ensemble. Suppose that the physical system $A$ and the reservoir $R$ together constitute an isolated composite system. The probability $P_{i}$ of finding the system $A$ in one particular microstate $i$ of energy $E_{i}$ can be found using the postulate of equal apriori probability since the composite system is isolated.

If $E_{0}$ represents the total energy of the composite system $A+R$, then the number of states accessible to $A+R$ is proportional to the number of states $\Omega_{i}\left(E_{0}-E_{i}\right)$ accessible to the reservoir. Therefore,

$$
P_{i}=\text { constant } \times \Omega_{i}\left(E_{0}-E_{i}\right)
$$


where the proportionality constant is determined by normalization. Since $A \ll R$ and $E_{i} \ll E_{0}, \ln \Omega_{i}\left(E_{0}-E_{i}\right)$ can be expanded into a Taylor series

$$
\ln \Omega_{i}\left(E_{0}-E_{i}\right)=\ln \Omega_{i}\left(E_{0}\right)-\left(\frac{\partial\left(\ln \Omega_{i}\right)}{\partial E}\right)_{E=E_{0}} E_{i}+\ldots
$$

Then $\beta$, defined as

$$
\beta=\left(\frac{\partial \ln \Omega_{i}}{\partial E}\right)_{E=E_{0}}
$$

is a characteristic of the reservoir. Using Eqs. 2.14 and $2.15, \beta^{-1}=k_{B} T$ where $T$ is the temperature $T$ of the reservoir. Since $A$ is in thermal equilibrium with the reservoir, $T$ is also the temperature of the system $A$. Then the number of accessible states to the composite system $A+R$ is approximated by truncating the Taylor expansion at the second term (first order term)

$$
\Omega_{i}\left(E_{0}-E_{i}\right) \cong \Omega_{i}\left(E_{0}\right) e^{-\beta E_{i}}
$$

Thus the probability $P_{i}$ is proportional to $e^{-\beta E_{i}}$, or after normalizing the probabilities for all possible states $i$ to one, i.e., $\sum_{i} P_{i}=1$, one obtains

$$
P_{i}=e^{-\beta E_{i}} / Z
$$

where

$$
Z=\sum_{i} e^{-\beta E_{i}}
$$


is called the partition function, which is the sum of the exponential factor $e^{-\beta E_{i}}$ over all states $i$. The partition function can also be defined as the volume in phase space occupied by the canonical ensemble. If the states form a continuum, then the summation in Eq. 2.21 becomes an integration.

Alternatively, the partition function can be written as

$$
Z=\sum_{E} \Omega(E) e^{-\beta E}
$$

where one can first sum over all the $\Omega(E)$ states in the energy range between $E$ and $E+\Delta E$ and then perform the summation over all such possible energy ranges. Note that the probability that a system has energy $E$ is $P(E) \propto \Omega(E) e^{-\beta E}$, while the probability that a system is in a state $i$ of energy $E_{i}$ is $P_{i} \propto e^{-\beta E_{i}}$. Since $\Omega(E)$ is an increasing function of $E$, and $e^{-\beta E}$ is an exponentially decreasing function of $E$, the product $\Omega(E) e^{-\beta E}$ has a maximum. The energy of the maximum $\tilde{E}$ is then the most probable energy of the system. If the maximum at $\tilde{E}$ is very sharp, then the partition function can be approximated by $Z \approx \Omega(\tilde{E}) e^{-\beta \tilde{E}}$, and

$$
\ln Z \approx \ln \Omega(\tilde{E})-\beta \tilde{E} .
$$

If we assume that $\tilde{E}=\langle E\rangle$, then $k_{B} T \ln Z=T\langle S\rangle-\langle E\rangle$, or

$$
F=\langle E\rangle-T\langle S\rangle=-k_{B} T \ln Z
$$

For a system in the canonical ensemble, one obtains the Helmholtz free energy from the partition function, which is obtained by summing over all possible states. On the other hand, for a system in the microcanonical ensemble, one derives the entropy by counting the number of accessible states. In thermodynamics, one uses the principles of minimization 
of Helmholtz free energy and maximization of entropy, respectively, in these two different cases.

The caloric curve in the canonical ensemble is given by

$$
E=T^{2} \frac{\partial \ln Z}{\partial T}
$$

The caloric curves for the canonical and microcanonical ensembles give similar results in the thermodynamic limit. However, they may differ considerably for small systems such as a nucleus. The caloric curves for small systems are discussed in detail in Appendix D.

\subsection{Radiative Strength Functions}

The radiative strength function (RSF) is considered a measure of the average electromagnetic nuclear properties. The RSF is important for understanding both nuclear structure and nuclear reactions. The transmission coefficients $T_{X L}$ for $\gamma$-ray transitions with energy $E_{\gamma}$ and multipolarity $X L$, which enter in reaction model calculations, are proportional to the corresponding strength functions $f_{X L}$

$$
T_{X L}\left(E_{\gamma}\right)=2 \pi E_{\gamma}^{2 L+1} f_{X L}\left(E_{\gamma}\right)
$$

The RSF was first estimated by Weisskopf in 1951 [24]. This estimate was based on an independent-particle model, and a single proton was assumed to be responsible for the transition. This energy-independent model is also known as the single-particle model.

Blatt and Weisskopf [25] also showed that the square of the $\gamma$-ray transition matrix element connecting compound states is proportional to the level spacing of the initial states with equal spin and parity. This led to a new description of the RSF for a transition of multipolarity $X L$ from an initial state $i$ to a final state $f$ in terms of the average partial radiative width $\left\langle\Gamma_{\gamma i f}\right\rangle$, level spacing $D_{i}$ of the initial states, and transition energy $E_{\gamma}$ 


$$
f_{X L}=\frac{\left\langle\Gamma_{\gamma i f}\right\rangle}{E_{\gamma}^{2 L+1} D_{i}} .
$$

The RSF of a nucleus is the sum over the strength functions $f_{X L}$ of all possible multipolarities $L$ and electric and magnetic type $X$. In general, all multipolarities and multipole types contribute, but the $\gamma$-ray emission probability with electric character is a factor of $10^{2}$ higher than the one with magnetic character. Furthermore, the photon emission with a multipolarity $L+1$ is less probable than the photon emission with a multipolarity $L$ by a factor of $10^{-6}$. Thus the $E 1$ and $M 1$ strengths contribute most to the RSF.

Most experimental information on RSF has been obtained from the study of photoabsorption cross sections $[26,27]$. Other methods involve investigation of primary $\gamma$ rays of different multipolarities [28] and two-step $\gamma$-ray cascades following thermal neutron capture $[29,30,31]$. However, these experimental methods provide radiative strengths mostly for high-energy transitions. It is difficult to measure the RSF between highly excited states because of the high level density. Therefore there is a serious lack of experimental information on the low-energy radiative decay strength.

\subsubsection{E1 Strength Function}

The simplest model for the $E 1$ photon strength is the energy-independent singleparticle model [24], and is given by (in units of cgs)

$$
f_{E 1}^{S P}=\frac{3}{4}\left(\frac{1}{\hbar c}\right)^{3} \frac{e^{2} R^{2}}{D_{s}}=C \frac{A^{2 / 3}}{D_{s}}
$$

where $e$ is the effective charge of a particle in a system of particles (for a neutron $e=-Z / A$ times the proton charge [32]), $R$ is the nuclear radius, $D_{s}$ is the spacing of the singleparticle states with $l=0$, and $C=6.8 \times 10^{-8} \mathrm{MeV}^{-2}$. A large amount of experimental data showed that the single-particle model overestimates the experimental $E 1$ strength 
function [33].

A more realistic approach makes use of the giant electric dipole resonance (GEDR). Giant dipole resonances are directly observed in photonuclear reactions. The principle of detailed balance $[33,34]$ for the $(n, \gamma)$ and $(\gamma, n)$ reactions, together with the assumption of the spin independence of the photon strength function, leads to the Lorentzian GEDR radiative strength function

$$
f_{E 1}\left(E_{\gamma}\right)=\frac{1}{3 \pi^{2} \hbar^{2} c^{2}} \frac{\sigma_{E 1} E_{\gamma} \Gamma_{E 1}^{2}}{\left(E_{\gamma}^{2}-E_{E 1}^{2}\right)^{2}+E_{\gamma}^{2} \Gamma_{E 1}^{2}}
$$

where $\sigma_{E 1}, \Gamma_{E 1}$, and $E_{E 1}$ are the standard giant electric dipole resonance parameters. This equation is valid for spherical nuclei. For deformed nuclei, the experimental data can be represented using a radiative strength function given by the sum of two Lorentzians.

Giant dipole resonances are most simply understood as the oscillations of neutrons against protons in the nucleus. The supposed independence of this collective motion from the intrinsic nuclear excitations leads to the important consequence (Brink's hypothesis [34]) that the giant resonances built on the ground state are similar in shape and size to those built on any excited state. A consequence of the Brink hypothesis is that the radiative strength function depends only on the energy of the transition, and is not dependent on the excitation energy.

Measurement of the primary $E 1$ radiation indicates that the GEDR model describes the experimental data much better than the single-particle model. Nevertheless, this model seems to be inadequate at energies close to the neutron binding energy [35].

It was also assumed that the tail of the Lorentzian describing the GEDR determines the strength function at lower energies. The only experimental data on the $E 1$ strength function between compound states with $\gamma$-ray energies below $2 \mathrm{MeV}$ have been obtained from the $(n, \gamma \alpha)$ reaction [36]. These data have shown that extrapolation of the GEDR fails to describe the experimental values of the $E 1$ strength function, and gives a finite value of the $E 1$ strength in the limit $E_{\gamma} \rightarrow 0$. As a result, more detailed models for the $E 1$ 
strength function were proposed by Zaretskij and Sirotkin [37], Kadmenskiü, Markushev, and Furman (KMF) [38], and Sirotkin [39]. These models rely on the theory of Fermi liquids and assume an energy and temperature dependent damping width of the GEDR

$$
\Gamma_{E 1}\left(E_{\gamma}, T\right)=\frac{\Gamma_{E 1}}{E_{E 1}^{2}}\left(E_{\gamma}^{2}+4 \pi^{2} T^{2}\right)
$$

where the nuclear temperature for thermal neutron capture is evaluated from the relation

$$
T=\sqrt{\left(B_{n}-E_{\gamma}-\Delta\right) / a}
$$

with $\Delta$ the pairing energy (equal to zero for odd-odd nuclei), and $a$ the Fermi-gas level density parameter. The strength function is given by

$$
f_{E 1}\left(E_{\gamma}\right)=\frac{1}{3 \pi^{2} \hbar^{2} c^{2}} \frac{0.7 \sigma_{E 1} \Gamma_{E 1}^{2}\left(E_{\gamma}^{2}+4 \pi^{2} T^{2}\right)}{E_{E 1}\left(E_{\gamma}^{2}-E_{E 1}^{2}\right)^{2}}
$$

The KMF model is frequently used in the description of experimental data, but needs further experimental verification.

\subsubsection{M1 Strength Function}

As in the case of the $E 1$ strength function, the single-particle model was the first model developed for the $M 1$ strength. Later, the $M 1$ strength function was also thought to be related to a corresponding giant resonance (see, e.g., Ref. [40]), which can be interpreted as the collective motion of spin-up nucleons against spin-down nucleons. This model is called the giant $M 1$ dipole resonance (GMDR) or spin-flip resonance $[40,41]$. The resonance parameters are usually taken from the global parameterization [42], which are $E_{\gamma}=41 A^{-1 / 3} \mathrm{MeV}, \Gamma_{M 1}=4 \mathrm{MeV}$, and the value of $\sigma_{M 1}$ is usually adjusted to fit experimental data at the neutron binding energy. The analysis of the primary $\gamma$ rays from 
$(n, \gamma)$ reactions [28] indicates that for $M 1$ radiation the GMDR model fits the experimental data better than the single-particle model.

The other magnetic dipole mode is the weakly collective isovector $M 1$ orbital resonance or the scissors mode (SM) $[43,44]$, and was first discovered in the heavy deformed nucleus ${ }^{156} \mathrm{Gd}$ in an $\left(e, e^{\prime}\right)$ experiment [45]. In contrast to certain electric excitations, magnetic excitations are weakly collective excitations.

An anomalous bump found in the $\gamma$-ray spectra of $(n, \gamma)$ and $(d, p \gamma)$ reactions [46, $47,48,49,50]$ has drawn special attention recently. This bump, also called a pygmy resonance, was also observed in the rare-earth nuclei in $\left({ }^{3} \mathrm{He}, \alpha\right)$ reactions $[51,52,53]$. Ongoing interpretations involve two possible characterizations of the pygmy resonance: An enhancement of the $E 1$ strength function or $M 1$ character connected to orbital $M 1$ strength - the scissors mode. A recent $(n, 2 \gamma)$ experiment is expected to determine the character of the pygmy resonance in ${ }^{172} \mathrm{Yb}$. 
$\prod_{\text {CHAPTER }} 3$

\section{Method to Extract Level Density and}

\section{Radiative Strength Function}

\section{Simultaneously}

\subsection{Introduction}

The method to extract simultaneously the level density and the radiative strength function (or $\gamma$-ray strength function) relies on the primary $\gamma$-ray spectra [54]. A primary $\gamma$ ray is the first $\gamma$ ray emitted from a level which is populated in a reaction. Details of the so-called subtraction method to obtain the experimental primary $\gamma$-ray matrix $P\left(E_{i}, E_{\gamma}\right)$ are given in Chapter 5; at this stage we will just assume that we are provided with this matrix. The $P\left(E_{i}, E_{\gamma}\right)$ matrix is a two-dimensional matrix, one axis is the excitation energy $E_{i}$ and the other axis is the primary $\gamma$-ray energy $E_{\gamma}$. Due to low statistics, the excitationenergy axis in this matrix is divided into $238-\mathrm{keV}$ wide bins. As a result, the extracted level density represents the average density of levels per 238-keV energy. By projecting an excitation energy $E_{i}$ bin onto the $E_{\gamma}$ axis, a primary $\gamma$-ray spectrum is obtained for that excitation-energy bin. The energy distribution of the primary $\gamma$ rays provides information on both the level density and the radiative strength function.

If the primary $\gamma$-ray spectrum is normalized to unity at each excitation energy, it 
represents the decay probability. Thus, the sum over the probabilities of emitting $\gamma$ rays from a minimum energy $E_{\gamma}^{\text {min }}$ to a maximum energy $E_{i}$ from a specific excitation energy $E_{i}$ bin is set equal to one

$$
\sum_{E_{\gamma}=E_{\gamma}^{\min }}^{E_{i}} P\left(E_{i}, E_{\gamma}\right)=1
$$

Figure 3.1 displays the normalized experimental $P\left(E_{i}, E_{\gamma}\right)$ matrix. Each pixel represents one matrix element, which gives the probability of emitting a $\gamma$ ray at the corresponding excitation energy. The matrix elements below $\mathrm{E}_{x}=4 \mathrm{MeV}$ of excitation energy are eliminated from the matrix since in this region the reaction is more likely to be direct than compound. Reactions other than compound nuclear violate the the main assumption of the subtraction method. The matrix elements below $\mathrm{E}_{\gamma}=1.5 \mathrm{MeV}$ are also excluded due to (i) electronic issues and (ii) problems associated with the subtraction method in obtaining the primary $\gamma$-ray spectra for low-energy $\gamma$ rays. For example, the yrast $\gamma$ rays are usually not properly subtracted, and any change in the spin population distribution between high and low excitations is not accounted for in the subtraction method.

\subsection{Ansatz}

\subsubsection{Axel-Brink Hypothesis}

The experimental normalized $P\left(E_{i}, E_{\gamma}\right)$ matrix is factorized according to the Axel-Brink hypothesis $[34,33]$, which states that the decay probability of a $\gamma$ ray from an excitation energy $E_{i}$ to a final energy $E_{f}$ is proportional to the product of the $\gamma$-ray transmission coefficient $T$ and the level density $\rho$ at the final level energy

$$
P\left(E_{i}, E_{\gamma}\right) \propto T\left(E_{\gamma}\right) \rho\left(E_{i}-E_{\gamma}\right)
$$

The final energy $E_{f}$ is given by the initial excitation energy minus the emitted $\gamma$-ray energy, as shown in Fig. 3.2. According to this hypothesis, the $P\left(E_{i}, E_{\gamma}\right)$ matrix is proportional 


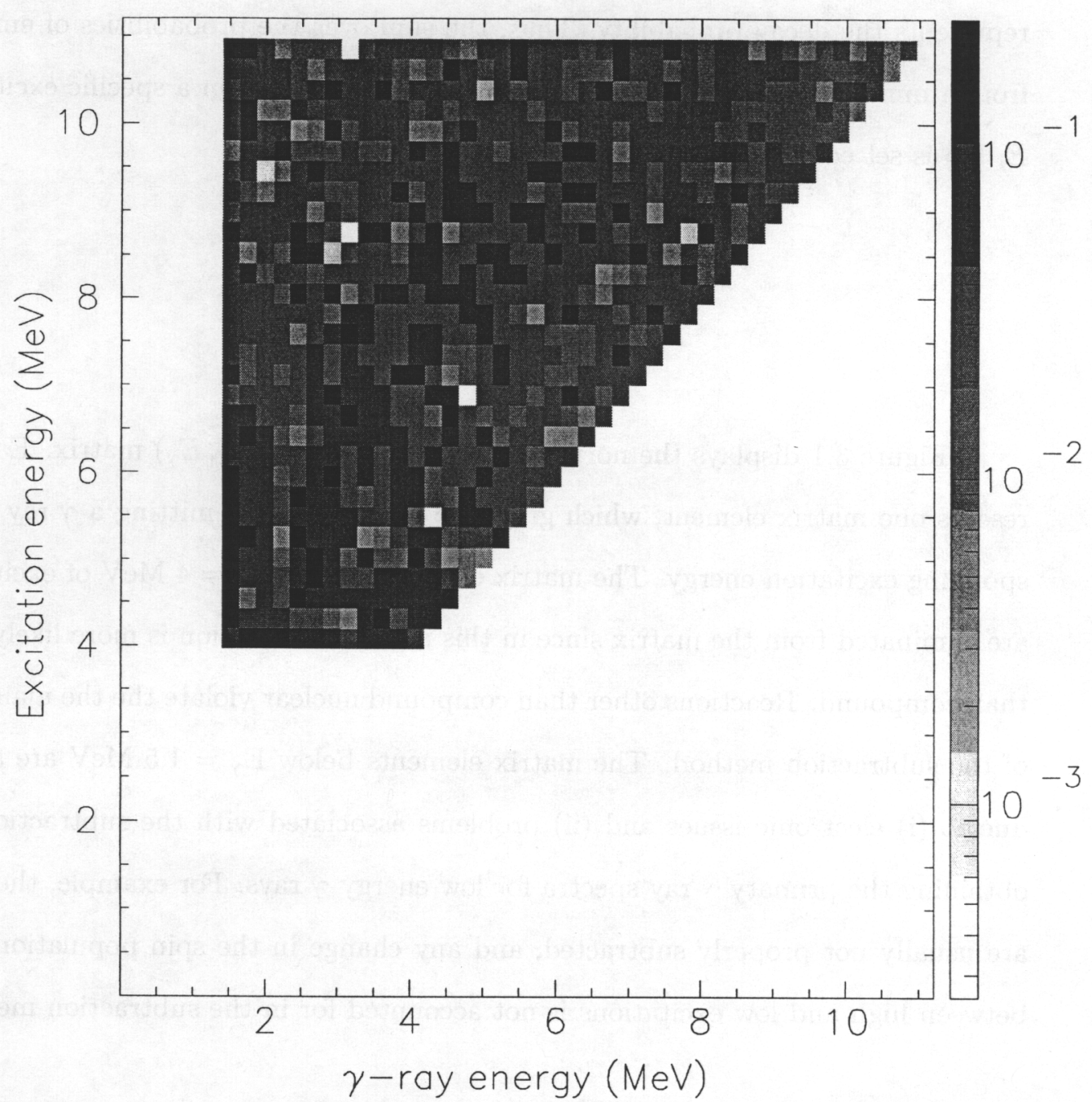

Figure 3.1: Normalized experimental primary $\gamma$-ray matrix for ${ }^{56} \mathrm{Fe}$.

to two functions of one variable: The $\gamma$-ray transmission coefficient is a function only of the $\gamma$-ray energy $E_{\gamma}$, and the level density is a function only of the final excitation energy. The function $T\left(E_{\gamma}\right)$ is given by the sum over all radiative strengths $f_{X L}\left(E_{\gamma}\right)$ weighted with $\gamma$-ray energies with multipolarities $X L$,

$$
T\left(E_{\gamma}\right)=\sum_{X L} 2 \pi E_{\gamma}^{2 L+1} f_{X L}\left(E_{\gamma}\right)
$$

A schematic representation of the $P\left(E_{i}, E_{\gamma}\right)$ matrix, shown in Fig. 3.3, helps to 


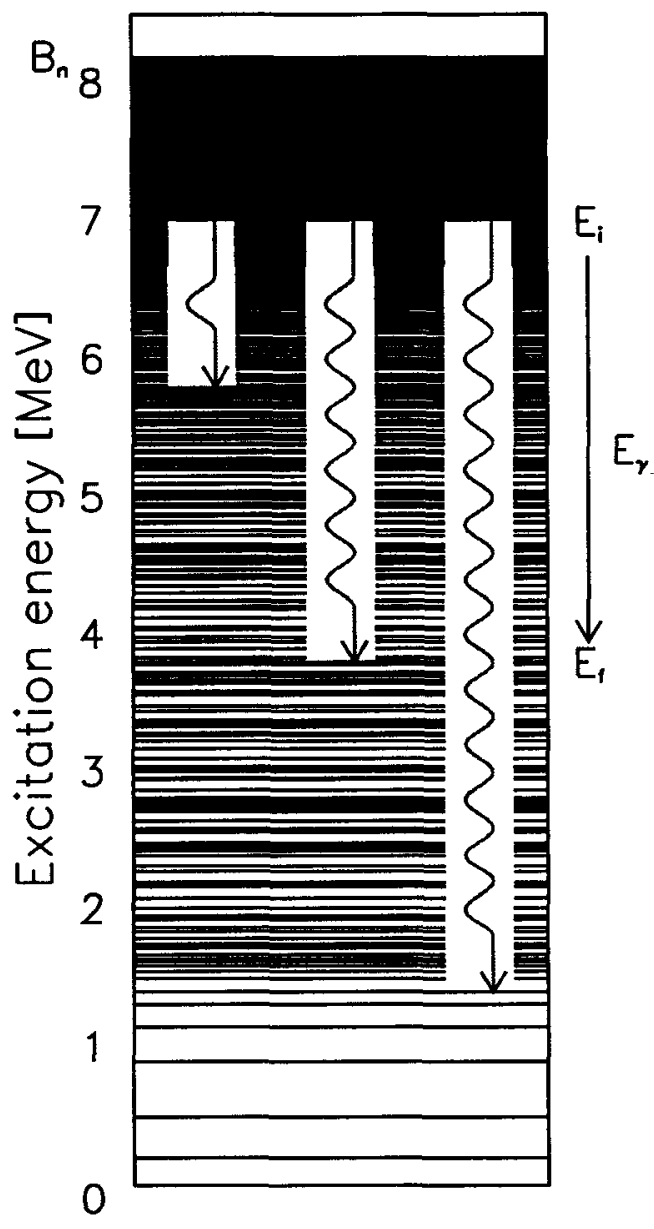

Figure 3.2: Statistical decay in the continuum energy region. The final energy is given by $E_{f}=E_{i}-E_{\gamma}$.

illustrate the application of Eq. 3.2 on the $P\left(E_{i}, E_{\gamma}\right)$ matrix elements. Each black dot represents one matrix element. Also notice that the maximum energy of a $\gamma$ ray emitted from a level is equal to the corresponding level energy. If Eq. 3.2 is written for each matrix element, then the matrix elements along the vertical lines in Fig. 3.3 depend on the function $T$ at the same $\gamma$-ray energy and the matrix elements along $45^{\circ}$ depend on the function $\rho$ at the same final excitation energy. Thus the experimental $P\left(E_{i}, E_{\gamma}\right)$ matrix elements are correlated with each other through the functions $\rho$ and $T$. 


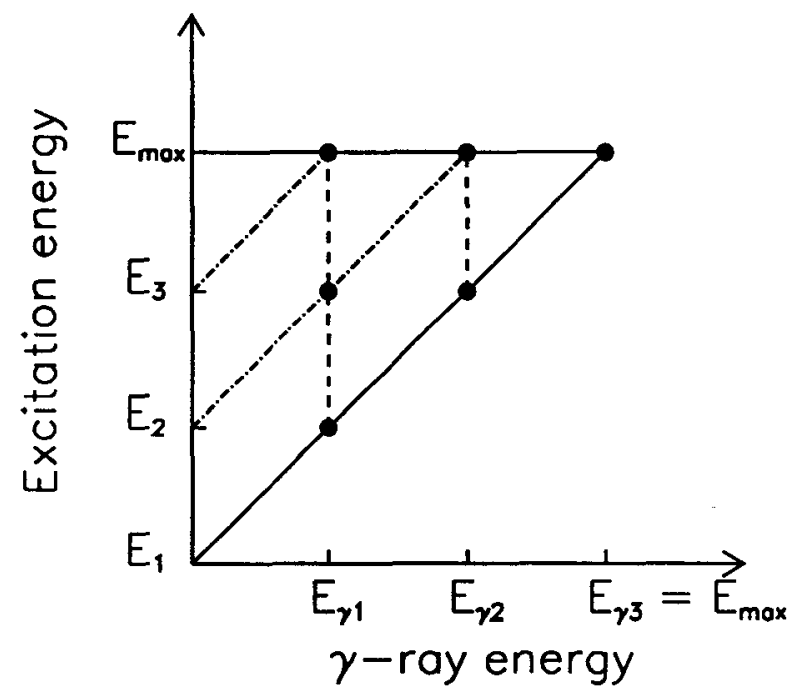

Figure 3.3: Schematic illustration of primary $\gamma$-ray matrix.

\subsubsection{Normalization of Eq. 3.2 at each $E_{x}$}

In order to fulfill Eq. 3.1, the right-hand side of Eq. 3.2 must be normalized to one at each excitation energy $E_{i}$. This gives

$$
P\left(E_{i}, E_{\gamma}\right)=\frac{T\left(E_{\gamma}\right) \rho\left(E_{i}-E_{\gamma}\right)}{\sum_{E_{\gamma}^{\prime}=E_{\gamma}^{\min }}^{E_{i}} T\left(E_{\gamma}^{\prime}\right) \rho\left(E_{i}-E_{\gamma}^{\prime}\right)}
$$

The two assumptions given by Eqs. 3.2 and 3.4 are the two physical inputs in this method, from which we would like to determine $\rho$ and $T$.

Unfortunately, neither $T$ nor $\rho$ is known a priori. Due to the functional form of Eq. 3.4, the number of solutions is infinite. We derive T's at all $\gamma$-ray energies and $\rho$ 's at all excitation energies by applying a least $\chi^{2}$ fit to the primary $\gamma$-ray data. These derived $T$ 's and $\rho$ 's together represent one solution that describes the primary $\gamma$-ray matrix. The solution obtained from this method is not necessarily the most physical solution. Therefore, we normalize the solution to known experimental data in order find the most probable solution. This normalization procedure is based on finding all other solutions from one arbitrary solution. Next, we describe how to find the other solutions, the least 
$\chi^{2}$ method, and the normalization procedure [54].

\subsubsection{Other Solutions of the Primary $\gamma$-ray Matrix}

Let $\tilde{\rho}$ and $\tilde{T}$ be another solution of the $P\left(E_{i}, E_{\gamma}\right)$ matrix, chosen as

$$
\begin{aligned}
\tilde{\rho}\left(E_{i}-E_{\gamma}\right) & =\rho\left(E_{i}-E_{\gamma}\right) g\left(E_{i}-E_{\gamma}\right) \\
\tilde{T}\left(E_{\gamma}\right) & =T\left(E_{\gamma}\right) f\left(E_{\gamma}\right)
\end{aligned}
$$

where the $\rho$ and $T$ together are one arbitrary solution obtained from the $\chi^{2}$ minimization, and $g\left(E_{i}-E_{\gamma}\right)$ and $f\left(E_{\gamma}\right)$ are the transformation functions to be determined. Then, the solution $\tilde{\rho}$ and $\tilde{T}$ must satisfy Eq. 3.4 such that

$$
P\left(E_{i}, E_{\gamma}\right)=\frac{\tilde{T}\left(E_{\gamma}\right) \tilde{\rho}\left(E_{i}-E_{\gamma}\right)}{\sum_{E_{\gamma}^{\prime}=E_{\gamma}^{\text {min }}}^{E_{i}} \tilde{T}\left(E_{\gamma}^{\prime}\right) \tilde{\rho}\left(E_{i}-E_{\gamma}^{\prime}\right)}
$$

Thus

$$
\frac{T\left(E_{\gamma}\right) \rho\left(E_{i}-E_{\gamma}\right)}{\sum_{E_{\gamma}^{\prime}=E_{\gamma}^{\min }}^{E_{i}} T\left(E_{\gamma}^{\prime}\right) \rho\left(E_{i}-E_{\gamma}^{\prime}\right)}=\frac{\tilde{T}\left(E_{\gamma}\right) \tilde{\rho}\left(E_{i}-E_{\gamma}\right)}{\sum_{E_{\gamma}^{\prime}=E_{\gamma}^{\min }}^{E^{\prime}} \tilde{T}\left(E_{\gamma}^{\prime}\right) \tilde{\rho}\left(E_{i}-E_{\gamma}^{\prime}\right)}
$$

By using this equation the transformation functions $g$ and $f$ are derived in Appendix A. Other solutions of Eq. 3.4 are then determined from the transformation equations given by

$$
\begin{aligned}
\tilde{\rho}\left(E_{i}-E_{\gamma}\right) & =\rho\left(E_{i}-E_{\gamma}\right) A \exp \left(\alpha\left(E_{i}-E_{\gamma}\right)\right) \\
\tilde{T}\left(E_{\gamma}\right) & =T\left(E_{\gamma}\right) B \exp \left(\alpha E_{\gamma}\right),
\end{aligned}
$$

where $A, B$, and $\alpha$ are free parameters. The transformation equations provide all other 
solutions from one arbitrary solution by adjusting the free parameters $A, B$, and $\alpha$. In other words, all solutions that describe the $P\left(E_{i}, E_{\gamma}\right)$ matrix with equal $\chi^{2}$ are related to each other by an exponential function, and thus by knowing one of the solutions one can compose all of the other solutions by choosing appropriate parameters $A, B$, and $\alpha$. Thus the method involves finding one solution and comparing it to known experimental data by employing Eq. 3.8 .

\subsection{Method}

One solution to Eq. 3.4 can be found by applying a least $\chi^{2}$ method. The reduced $\chi^{2}$ is defined as

$$
\chi^{2}=\frac{1}{N_{\text {free }}} \sum_{E_{i}=E_{i}^{\min }}^{E_{i}^{\max }} \sum_{E_{\gamma}=E_{\gamma}^{\min }}^{E_{i}}\left(\frac{P\left(E_{i}, E_{\gamma}\right)-P_{\text {exp }}\left(E_{i}, E_{\gamma}\right)}{\triangle P_{\text {exp }}\left(E_{i}, E_{\gamma}\right)}\right)^{2}
$$

where $N_{\text {free }}$ is the number of degrees of freedom, and is found by

$$
N_{\text {free }}=\operatorname{ch}(P)-\operatorname{ch}(\rho)-\operatorname{ch}(T) .
$$

where $c h$ indicates the number of data points in the respective spectra. $\operatorname{ch}(P)$ is simply the area of the primary $\gamma$-ray matrix, shown in Fig. 3.1, and $\operatorname{ch}(\rho)$ and $\operatorname{ch}(T)$ are the number of excitation-energy bins. Then the reduced $\chi^{2}$ is minimized with respect to $T$ at every $\gamma$-ray energy, and with respect to $\rho$ at every excitation energy according to

$$
\begin{gathered}
\frac{\partial}{\partial T\left(E_{\gamma}\right)} \chi^{2}=0, \\
\frac{\partial}{\partial \rho\left(E_{i}-E_{\gamma}\right)} \chi^{2}=0 .
\end{gathered}
$$


If we denote the number of excitation-energy bins by $n$, which is also equal to the number of $E_{\gamma}$ bins, there are a total of $2 n$ equations, $n$ equations from 3.11 and $n$ equations from 3.12. However, only $2 n-3$ of these equations are independent. Thus, there are $2 n-3$ equations, but $2 n$ unknowns. Since these equations are not a linear system of equations, they cannot be solved analytically. One must solve them numerically by introducing three arbitrary values for $\rho$ and/or $T$. Thus by the choice of these three values arbitrarily each time we obtain a different solution. As proven with the transformation equations, again we see that the correct solution can be obtained only if we introduce three variables correctly.

The set of equations obtained from the $\chi^{2}$ minimization is very tedious to solve. Therefore, an iteration procedure is implemented in the program called RHOSIGCHI. The program starts with an arbitrary solution, and in each iteration calculates a new $\rho$ and $T$ using values from the previous iteration and the $\chi^{2}$. The iteration continues until the $\chi^{2}$ is minimized.

\subsubsection{Zeroth-order Estimate}

Since the choice of one solution is completely arbitrary in the transformation equations, we can chose a solution which is convenient as a starting point. Thus $\rho^{(0)}=1$ is chosen as a zeroth-order estimate. Then, the zeroth-order estimate for $T^{(0)}\left(E_{\gamma}\right)$ is calculated from Eq. 3.4

$$
P\left(E_{i}, E_{\gamma}\right)=\frac{T^{(0)}\left(E_{\gamma}\right)}{\sum_{E_{\gamma}=E_{\gamma}^{\min }}^{E_{i}} T^{(0)}\left(E_{\gamma}\right)}
$$

Summing over all of the excitation energies gives

$$
\sum_{E_{i}=\max \left(E_{i}^{\min }, E_{\gamma}\right)}^{E_{i}^{\max }} P\left(E_{i}, E_{\gamma}\right)=T^{(0)}\left(E_{\gamma}\right) \sum_{E_{i}=\max \left(E_{i}^{\min }, E_{\gamma}\right)}^{E_{i}^{\max }} \frac{1}{\sum_{E_{\gamma}=E_{\gamma}^{\min }}^{E_{i}} T^{(0)}\left(E_{\gamma}\right)}
$$

The sum on the right-hand side can be set to unity for convenience, then one obtains 


$$
T^{(0)}\left(E_{\gamma}\right)=\sum_{E_{i}=\max \left(E_{i}^{\min }, E_{\gamma}\right)}^{E_{i}^{\max }} P\left(E_{i}, E_{\gamma}\right)
$$

\subsubsection{Higher-order Estimates.}

The derivation of the higher order estimates from the $\chi^{2}$ minimization (see Eqs. 3.11 and 3.12) is tedious and is given in Appendix B. They are

$$
\begin{gathered}
T\left(E_{\gamma}\right)=\frac{\sum_{E_{i}=\max \left(E_{i}^{\text {min }}, E_{\gamma}\right)}^{E_{\text {max }}} \rho\left(E_{i}-E_{\gamma}\right) \varphi\left(E_{i}, E_{\gamma}\right)}{\sum_{E_{i}=\max \left(E_{i}^{\min }, E_{\gamma}\right)}^{E^{\max }} \rho^{2}\left(E_{i}-E_{\gamma}\right) \psi\left(E_{i}, E_{\gamma}\right)} \\
\rho\left(E_{f}\right)=\frac{\sum_{E_{i}=\max \left(E_{i}^{\min }, E_{f}+E_{\gamma}^{\min }\right)}^{E_{\max }^{\max }} T\left(E_{i}-E_{f}\right) \varphi\left(E_{i}, E_{i}-E_{f}\right)}{\sum_{E_{i}=\max \left(E_{i}^{\min }, E_{f}+E_{\gamma}^{\min }\right)}^{E^{\max }} T^{2}\left(E_{i}-E_{f}\right) \psi\left(E_{i}, E_{i}-E_{f}\right)}
\end{gathered}
$$

where

$$
\begin{gathered}
\varphi\left(E_{i}, E_{\gamma}\right)=\frac{a\left(E_{i}\right)}{\left[s\left(E_{i}\right)\right]^{3}}-\frac{b\left(E_{i}\right)}{\left[s\left(E_{i}\right)\right]^{2}}+\frac{P_{e x p}\left(E_{i}, E_{\gamma}\right)}{s\left(E_{i}\right)\left[\triangle P_{e x p}\left(E_{i}, E_{\gamma}\right)\right]^{2}} \\
\psi\left(E_{i}, E_{\gamma}\right)=\frac{1}{\left[s\left(E_{i}\right) \triangle P_{\exp }\left(E_{i}, E_{\gamma}\right)\right]^{2}}
\end{gathered}
$$

and 


$$
\begin{aligned}
& a\left(E_{i}\right)=\sum_{E_{\gamma}=E_{\gamma}^{\min }}^{E_{i}}\left[\frac{T\left(E_{\gamma}\right) \rho\left(E_{i}-E_{\gamma}\right)}{\triangle P_{\exp }\left(E_{i}, E_{\gamma}\right)}\right]^{2} \\
& b\left(E_{i}\right)=\sum_{E_{\gamma}=E_{\gamma}^{\text {min }}}^{E_{i}} \frac{T\left(E_{\gamma}\right) \rho\left(E_{i}-E_{\gamma}\right) P_{\text {exp }}\left(E_{i}, E_{\gamma}\right)}{\left[\triangle P_{\text {exp }}\left(E_{i}, E_{\gamma}\right)\right]^{2}} \\
& s\left(E_{i}\right)=\sum_{E_{\gamma}=E_{\gamma}^{\min }}^{E_{i}} T\left(E_{\gamma}\right) \rho\left(E_{i}-E_{\gamma}\right) .
\end{aligned}
$$

The iteration process starts with the zeroth-order estimates $\rho^{(0)}$ and $T^{(0)}$. These estimates are kept the same for the level density at all of the excitation energies and for the $\gamma$-ray transmission coefficient at all of the $\gamma$-ray energies. Within one iteration, first $a\left(E_{i}\right), b\left(E_{i}\right)$, and $s\left(E_{i}\right)$ are calculated using the estimates for $\rho$ and $T$ from the previous iteration. Using these functions, the matrices $\varphi\left(E_{i}, E_{\gamma}\right)$ and $\psi\left(E_{i}, E_{\gamma}\right)$ are calculated. Then new estimates for $\rho$ and $T$ are calculated using Eqs. 3.16 and 3.17. Figure 3.4 shows how the summations in Eqs. 3.16 and 3.17 are performed. The shaded areas are usually excluded from the matrix. In this figure, the region of $E_{\gamma}<1 \mathrm{MeV}$ and $E_{i}<4 \mathrm{MeV}$ is excluded. If the $\gamma$-ray energy is between 1 and $4 \mathrm{MeV}$, the sum in calculating the $F\left(E_{\gamma}\right)$ starts from $E_{i}=4 \mathrm{MeV}$ (upper left panel of Fig. 3.4). If $E_{\gamma}>4 \mathrm{MeV}$, the sum starts from $E_{i}=E_{\gamma}$ (upper right panel). Similarly, if the final excitation energy in calculating the level density $\rho\left(E_{f}\right)$ falls between $3 \mathrm{MeV}$ and $B_{n}-1 \mathrm{MeV}$, the sum starts from $E_{f}+E_{\gamma}^{\text {min }}$ (lower left panel). If $E_{f}<3 \mathrm{MeV}$, the sum starts from $E_{i}^{m i n}=4 \mathrm{MeV}$.

\subsection{Convergence Properties}

The $\chi^{2}$ for most data points converges within 10 iterations, where the maximum number of iterations is set to 50 . However, some data points, usually those with high $E_{\gamma}$ and $E_{i}$ energies which have fewer counts, may oscillate between two values without converging. In order to improve the convergence properties the maximum variation for the functions $\rho$ and $T$ is restricted within one iteration to a certain percentage $p$. If the variation lies outside 

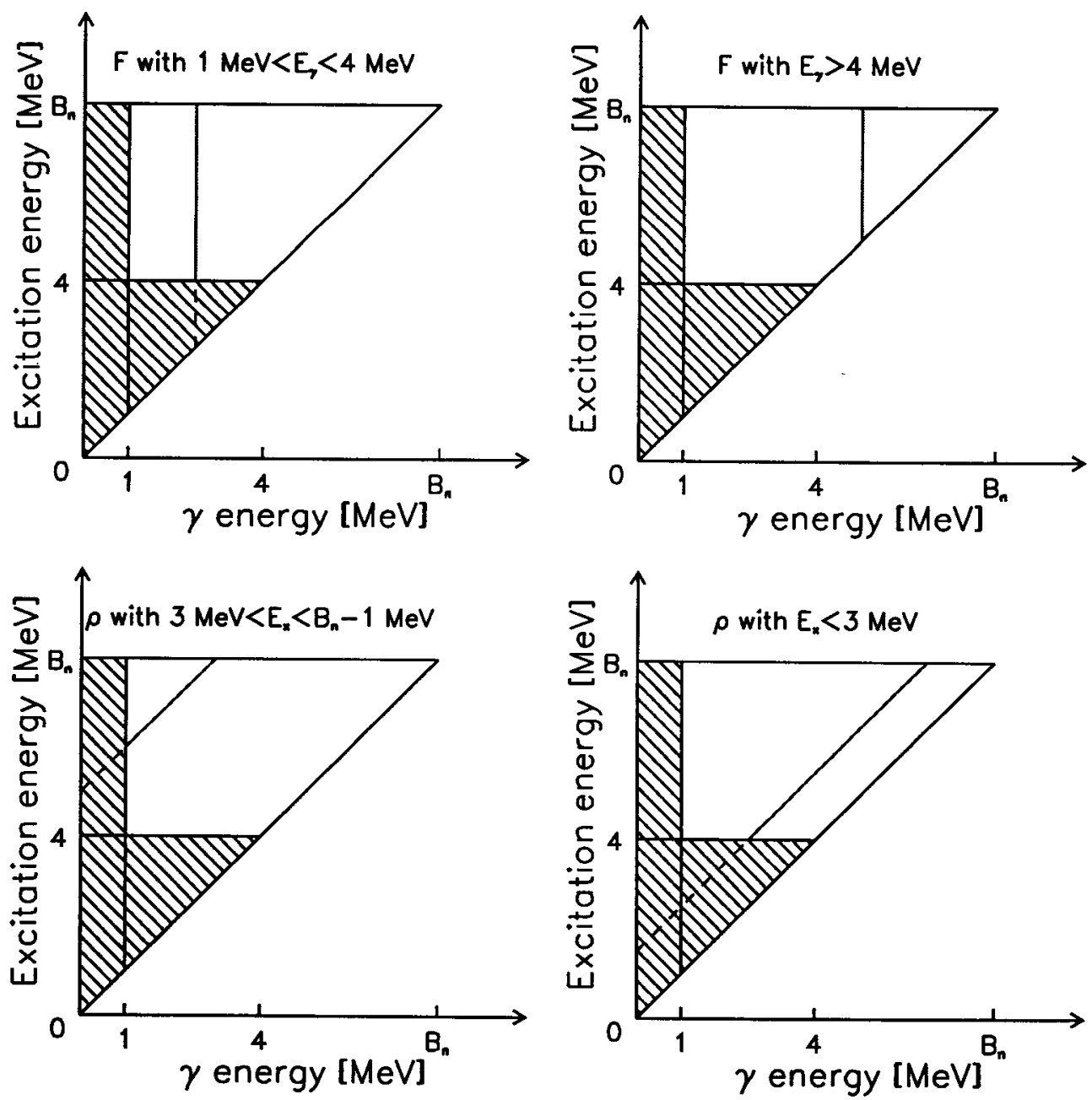

Figure 3.4: Schematic illustration of performing the sum in Eqs. 3.16 and 3.17.

$$
\frac{\text { old }}{1+p / 100} \leq \text { new } \leq \text { old } \times(1+p / 100)
$$

then the value is set to the closest boundary. A small $p$ would increase the convergence properties, but also the execution time. A large $p$ would decrease the execution time, but affect the accuracy of the solution. Therefore, the percentage $p$ is implemented such that it becomes smaller as a function of the number of the iterations, as listed in Table 3.1. 
The value of $p$ can be changed since the reliability of the method does not depend on the choice of the value of $p$.

Table 3.1: Percentage $p$ as a function of the number of iterations. $p$ decreases from $20 \%$ to $1 \%$. The maximum variation that any point can have within 50 iterations is approximately a factor of 11.

\begin{tabular}{|c|c|c|c|}
\hline Iteration & $p$ & $\begin{array}{l}\text { Number of } \\
\text { iterations }\end{array}$ & Max. variation \\
\hline $1-5$ & 20 & 5 & $1.2^{5} \approx 2.49$ \\
\hline $6-12$ & 10 & 7 & $1.1^{7} \approx 1.95$ \\
\hline $13-21$ & 5 & 9 & $1.05^{9} \approx 1.55$ \\
\hline $22-30$ & 2.5 & 9 & $1.025^{9} \approx 1.25$ \\
\hline \multirow[t]{2}{*}{$31-50$} & 1 & 20 & $1.01^{20} \approx 1.22$ \\
\hline & & $\sum=50$ & $\Pi \approx 11.46$ \\
\hline
\end{tabular}

\subsection{Error Calculations}

Error estimation for the functions $\rho$ and $T$ is very difficult, and requires first finding the error in the primary $\gamma$-ray matrix $P\left(E_{i}, E_{\gamma}\right)$. This matrix is obtained from the raw data after the unfolding and subtraction procedures. The error propagation through these methods is very tedious and has never been performed. Therefore, the error in the $P\left(E_{i}, E_{\gamma}\right)$ matrix is calculated by a different procedure:

$$
\Delta P=2 \sqrt{\left(M_{1}+M_{2}\right) P}
$$

where $M_{1}$ is the multiplicity of the first- and higher-order $\gamma$ rays, and $M_{2}$ is the multiplicity of the second- and the higher-order $\gamma$ rays at one excitation energy. These multiplicities are roughly given by

$$
\begin{aligned}
& M_{1}=\max \left(1, M\left(E_{i}\right)\right) \\
& M_{2}=\max \left(0, M\left(E_{i}\right)-1\right)
\end{aligned}
$$


The multiplicity $M\left(E_{i}\right)$ is obtained by a fit to the experimental data in Ref. [55]

$$
M\left(E_{i}\right)=0.42+4.67 \times 10^{-4} E_{i}-1.29 \times 10^{-8} E_{i}^{2},
$$

$E_{i}$ is given in keV. The motivation behind Eq. 3.22 is as follows: The number of counts in the primary $\gamma$-ray spectrum is of the order of $M_{1} P$, and in the second- and higher-order $\gamma-$ ray spectrum is of the order of $M_{2} P$. The errors in these spectra are approximately $\sqrt{M_{1} P}$ and $\sqrt{M_{2} P}$, respectively. Since the primary $\gamma$-ray spectra are obtained by subtraction of these two spectra, the error in the primary $\gamma$-ray spectrum is calculated from the error propagation assuming that these errors are independent, which gives $\sqrt{\left(M_{1}+M_{2}\right) P}$. The factor of two is included in Eq. 3.22 due to the uncertainty in the unfolding procedure. This factor is roughly calculated from the ratio of the solid angle covered by the CACTUS detector array (15\%) to its photopeak efficiency (7\%). In addition, two minor corrections must be considered.

The errors for low-energy $\gamma$ rays need to be increased since the spin effect is considered to be larger for low $\gamma$-ray energies. This is performed as follows: For each excitation energy bin, the channel with the maximum number of counts, denoted by $x_{m}$, is identified (this channel usually corresponds to a $\gamma$-ray energy between 2 and $3 \mathrm{MeV}$ ). This channel also has the highest error $\sigma_{m}$. Then the errors for the channels below $x_{m}$ is replaced by

$$
\sigma=\sigma_{m} \times\left(1+\frac{x_{m}-x}{x_{m}}\right)
$$

This formula has been tested on several primary $\gamma$-ray spectra, and gives reasonable systematic errors.

The second correction is due to the unfolding procedure. The ratio of the photopeak efficiency to the solid angle covered by the $\mathrm{NaI}(\mathrm{Tl})$ detectors decreases for high $\gamma$-ray energies; thus the counts at these energies are multiplied by significant factors in the unfolding procedure. Since some channels at high energies might have very few or zero 
counts, the difference in counts between two neighboring channels may be very large. Since the estimated errors are proportional to the square root of the number of counts, these errors do not reflect their statistical significance. Therefore, if the error drops more than a factor of two between two neighboring bins, the error in the higher-energy bin is set to $50 \%$ of the error of the previous bin. This procedure affects only a small percentage of channels.

The error propagation for the extraction method is also tedious. Therefore, a simulation technique is applied. First, a statistical fluctuation is added into the primary $\gamma$-ray matrix: For every matrix element a random number between zero and one is chosen. Then a new number of counts for every matrix element is calculated as

$$
r=\int_{-\infty}^{b} \exp \left(-\frac{(x-a)^{2}}{2 \sigma^{2}}\right) d x
$$

where $a$ is the number of counts and $\sigma$ is the error in the matrix element. Then $a$ is replaced by the new number number of counts $b$ that includes the statistical fluctuations. The functions $\rho$ and $T$ are extracted using the new matrix weighted by statistical fluctuations. These functions are calculated 100 times in order to obtain good statistics. The errors in the functions $\rho$ and $T$ are then calculated by

$$
\begin{aligned}
& \Delta \rho=\frac{1}{\sqrt{100}} \sqrt{\sum_{i=1}^{100}\left[\rho_{i}\left(E_{f}\right)-\rho\left(E_{f}\right)\right]^{2}} \\
& \Delta T=\frac{1}{\sqrt{100}} \sqrt{\sum_{i=1}^{100}\left[T_{i}\left(E_{\gamma}\right)-T\left(E_{\gamma}\right)\right]^{2}} .
\end{aligned}
$$

\subsection{Normalization of the Level Density to Experimental Data}

The level density for each excitation energy obtained above is only one solution: there are infinitely many equally good solutions, which have the same $\chi^{2}$. The average slope 
and magnitude of the solution obtained from the $\chi^{2}$ minimization depends on the initial choices of the functions $\rho$ and $T$. One can find the most physical solution by comparing this solution to known data. For the normalization procedure we need two anchor points in order to fix both the slope and the magnitude of the solution.

We compare the inferred level density to the known discrete levels per excitationenergy bin at low excitation energies and to the level density determined from the known neutron-resonance data at the neutron binding energy $B_{n}$. For the level density at $B_{n}$ we use the Fermi-gas model [6]

$$
\rho(U, J)=\frac{\sqrt{\pi}}{12} \frac{\exp (2 \sqrt{a U})}{a^{1 / 4} U^{5 / 4}} \frac{(2 J+1) \exp \left(-(J+1 / 2)^{2} / 2 \sigma^{2}\right)}{2 \sqrt{2 \pi} \sigma^{3}}
$$

for both parities and for a given spin $J$, and for all spins and parities

$$
\rho(U)=\frac{\sqrt{\pi}}{12} \frac{\exp (2 \sqrt{a U})}{a^{1 / 4} U^{5 / 4}} \frac{1}{\sqrt{2 \pi} \sigma}
$$

where $\sigma$ is the spin cut-off parameter and $a$ is the level density parameter. The neutronresonance spacing can be written as

$$
\frac{1}{D}=\frac{1}{2}\left(\rho\left(U_{n}, J=I+1 / 2\right)+\rho\left(U_{n}, J=I-1 / 2\right)\right)
$$

where $I$ is the spin of the target nucleus in a neutron resonance experiment, $U_{n}$ is defined as the binding energy $B_{n}$ minus the pairing energy, $U_{n}=B_{n}-P$, with the assumption that both parities contribute equally to the level density at $B_{n}$. Combining Eqs. $3.29-$ 3.31 yields the level density at $B_{n}$

$$
\rho\left(U_{n}\right)=\frac{2 \sigma^{2}}{D} \frac{1}{(I+1) \exp \left(-(I+1)^{2} / 2 \sigma^{2}\right)+I \exp \left(-I^{2} / 2 \sigma^{2}\right)}
$$


where $\sigma^{2}$ is calculated using Ref. [6]

$$
\sigma^{2}=0.0888 \sqrt{a U_{n}} A^{2 / 3}
$$

and $A$ is the mass number of the nucleus. It is assumed that $\sigma^{2}$ has an error of about $\approx$ $10 \%$ due to shell effects [6].

Unfortunately, the level density cannot be compared directly to the level density at $B_{n}$, since we extract the level density up to $B_{n}-1.5 \mathrm{MeV}$ due to the omission of the $\gamma$ rays below $1.5 \mathrm{MeV}$. Therefore the experimental level density is extrapolated to $B_{n}$ using the Fermi-gas level density, by combining Eqs. 3.30 and 3.33,

$$
\rho(U)=\frac{1}{12 \sqrt{0.1776} A^{1 / 3}} \frac{\exp (2 \sqrt{a U})}{a^{1 / 2} U^{3 / 2}}
$$

This is performed by adjusting the parameters $A$ and $\alpha$ of the transformation given by Eq. 3.8 such that the data fit the Fermi-gas level density in an excitation energy interval between $1.5 \mathrm{MeV}$ and $2.5 \mathrm{MeV}$ below the neutron binding energy. The level density is also adjusted to fit the known discrete levels at low excitation. More details will be given in Chapter 6.

\subsection{Testing the Method on Theoretical Spectra}

The method has been tested on theoretically calculated primary $\gamma$-ray matrices $P_{t h}\left(E_{i}, E_{\gamma}\right)$. The theoretical $P_{t h}\left(E_{i}, E_{\gamma}\right)$ is constructed by multiplying a level density expression for $\rho$ by a $\gamma$-ray transmission coefficient expression for $T$. The back-shifted Fermi-gas formula is chosen for the level density

$$
\rho(U)=C U^{-3 / 2} \exp (2 \sqrt{a U})
$$



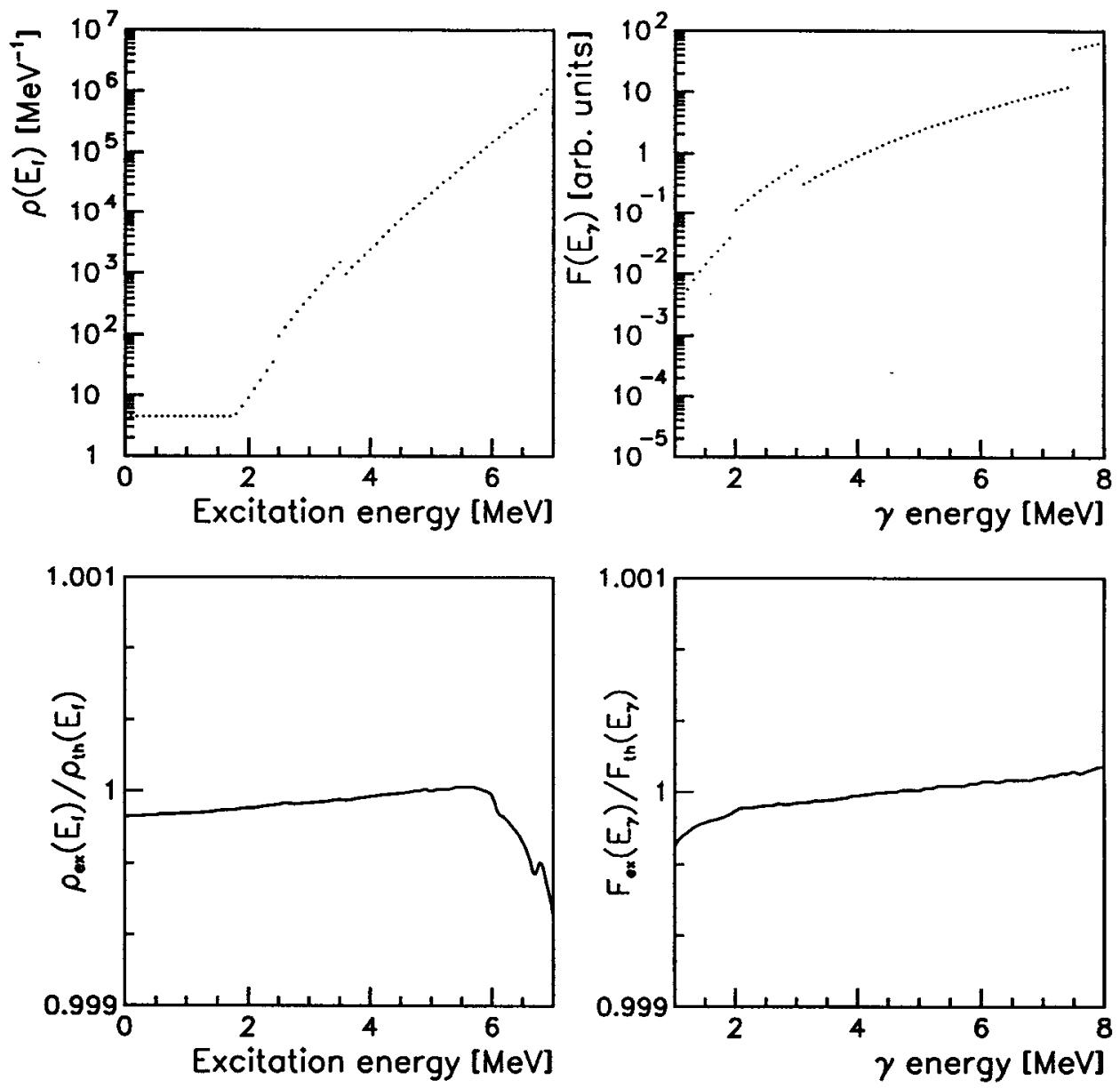

Figure 3.5: Theoretical level density (top, left) and radiative strength function (top, right) used to calculate the primary $\gamma$-ray spectra. The constant ratio of the extracted and theoretical values (lower panels) proves the reliability of the method.

where $U$ is the final excitation energy minus the pairing energy. Below the minimum at $U=9 / 4 a$, a constant level density was used. The $\gamma$-ray transmission coefficient was chosen as

$$
T\left(E_{\gamma}\right)=C E_{\gamma}^{4.2}
$$


In addition, a fine structure was imposed on both functions $\rho$ and $T$ by scaling several $\sim 1-\mathrm{MeV}$ broad intervals with factors around $1.5-5$. These functions are shown in the upper half in Fig. 3.5. By applying the $\chi^{2}$ minimization, the functions $\rho$ and $T$ were extracted from the theoretical $P_{t h}\left(E_{i}, E_{\gamma}\right)$ matrix using the excitation energy interval of $E_{i}=4-8 \mathrm{MeV}$ and excluding all $\gamma$ rays below $E_{\gamma}=1 \mathrm{MeV}$. Then the extracted $\rho$ and $T$ were adjusted using the transformation given in Eq. 3.8. The ratio of the extracted and theoretical functions is shown in the lower panels of Fig. 3.5. The deviation from the input functions was found to be smaller than one per thousand in the energy range of both functions (see Fig. 3.5), which indicates the reliability of the method.

\subsection{Application of the Method to ${ }^{56} \mathrm{Fe}$ and ${ }^{57} \mathrm{Fe}$ Spectra}

The primary $\gamma$-ray spectra for ${ }^{56} \mathrm{Fe}$ and ${ }^{57} \mathrm{Fe}$ are obtained from the $\left({ }^{3} \mathrm{He}, \alpha\right)$ and $\left({ }^{3} \mathrm{He}^{3} \mathrm{He}^{\prime}\right)$ reactions, respectively. The details of the experiment are given in the next chapter. In Fig. 3.6 and 3.7, the normalized experimental primary $\gamma$-ray spectra for ${ }^{56} \mathrm{Fe}$ and ${ }^{57} \mathrm{Fe}$, respectively, are shown with data points at ten different excitation-energy bins. The lines are the calculated primary $\gamma$-ray spectra, obtained by multiplying the extracted $\rho$ 's and $T$ 's according to Eq. 3.4. The experimental and calculated spectra agree very well, which justifies the assumption that the primary $\gamma$-ray spectrum can be factorized according to the Axel-Brink hypothesis. 


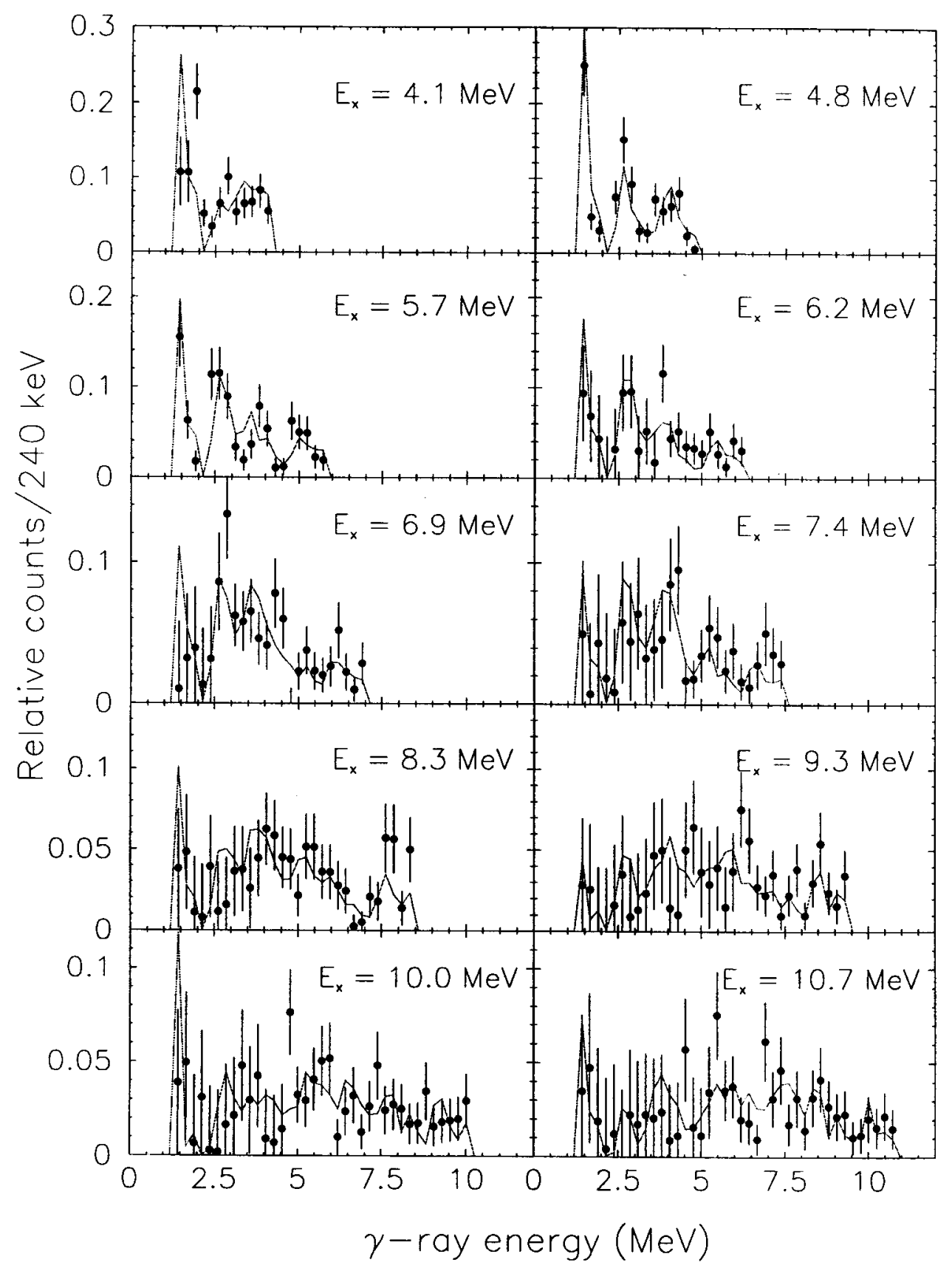

Figure 3.6: Experimental (data points) and calculated (solid line) primary $\gamma$-ray spectra for different excitation energies in ${ }^{56} \mathbf{F e}$. 


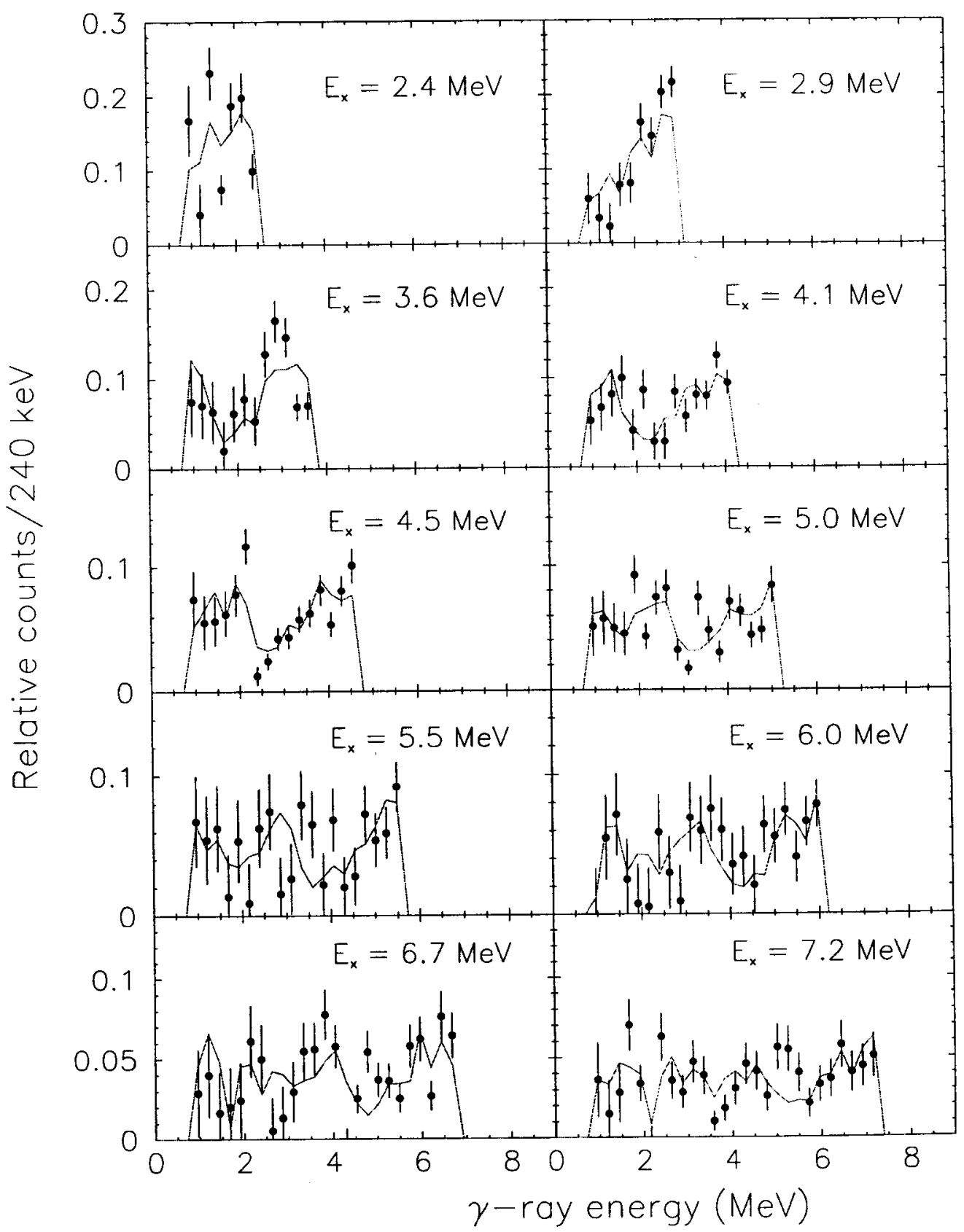

Figure 3.7: Same as Fig. 3.6 in ${ }^{57}$ Fe. 
$\prod_{\text {CHAPTER }}$

\section{Experimental Details}

\subsection{Introduction}

The experiment was performed at the Oslo Cyclotron Laboratory at the University of Oslo in December of 2000. A 45- $\mathrm{MeV}^{3} \mathrm{He}$ beam was delivered from a Scanditronix model MC35 cyclotron on a self-supporting ${ }^{57} \mathrm{Fe}$ target with a thickness of $3.38 \mathrm{mg} / \mathrm{cm}^{2}$. The target was isotopically enriched to $94.7 \%$ with impurities as listed in Table 4.1 .

Table 4.1: Impurities in the ${ }^{57} \mathrm{Fe}$ target.

\begin{tabular}{cc}
\hline \hline Isotope & Abundance (\%) \\
\hline${ }^{58} \mathrm{Fe}$ & 2.1 \\
${ }^{56} \mathrm{Fe}$ & 3.2 \\
\hline \hline
\end{tabular}

In order to prevent pile up in the detectors, the beam current in the MC35 cyclotron was limited to a few nanoamperes. The MC35 cyclotron can accelerate protons, deuterons, ${ }^{3} \mathrm{He}$, and $\alpha$ particles. The maximum energies of these ions are listed in Table 4.2. The experiment was performed for a week. The reaction $\gamma$ rays and particles were measured by the CACTUS multidetector array. The reactions ${ }^{57} \mathrm{Fe}\left({ }^{3} \mathrm{He}, \alpha\right){ }^{56} \mathrm{Fe}$ and $\left.{ }^{57} \mathrm{Fe}\left({ }^{3} \mathrm{He},{ }^{3} \mathrm{He}\right)^{\prime}\right)^{57} \mathrm{Fe}$ are the focus for the present analysis. The experimental equipment is described in detail in the following sections. 
Table 4.2: Beam types and their maximum energies produced at the Oslo Cyclotron Laboratory.

\begin{tabular}{llc}
\hline \hline Type of beam & & Max. energy $(\mathrm{MeV})$ \\
\hline Protons & ${ }^{1} \mathrm{H}^{+}$ & 35 \\
Deuteron & ${ }^{2} \mathrm{H}^{+}$ & 18 \\
Helium-3 & ${ }^{3} \mathrm{He}^{++}$ & 47 \\
$\alpha$ & ${ }^{4} \mathrm{He}^{++}$ & 35 \\
\hline
\end{tabular}

\subsection{CACTUS Multidetector Array}

The CACTUS detector array consists of $285^{\prime \prime} \times 5^{\prime \prime} \mathrm{NaI}(\mathrm{Tl})$ and three HPGe detectors for measurements of the $\gamma$ rays, and eight silicon detectors for charged-particle detection. The $\mathrm{NaI}(\mathrm{Tl})$ detectors in the array are mounted in a spherical frame with a radius of 250 $\mathrm{mm}$. The distance between the target position and the detectors is $220 \mathrm{~mm}$. The detector configuration is illustrated schematically in Fig. 4.1. The position angles of the $\mathrm{NaI}(\mathrm{Tl})$ detectors are listed in Table 4.3. $\theta$ is defined as the angle for a detector with respect to the beam direction, and the angle $\phi$ increases in the clockwise direction in the plane. Only one HPGe detector was used for the present experiment. The HPGe detector, which is not shown in Fig. 4.1, is also implemented in the detector frame in the position listed in Table 4.4.

The total solid angle for the $\mathrm{NaI}(\mathrm{Tl})$ detectors is calculated to be $17.7 \%$ of $4 \pi$ from $\Omega=\frac{N A}{4 \pi R^{2}}$, where $N=28$ is the number of detectors, $A=\pi a^{2}$ is the collimated front area of one detector with a radius of $a=35 \mathrm{~mm}$, and $R=220 \mathrm{~mm}$ is the distance of the detectors from the target.

At the end of the beam tube, a vacuum chamber is placed inside the detector frame. Eight Si particle telescopes are positioned in the vacuum chamber approximately $5 \mathrm{~cm}$ from the target on a plastic ring with an angle of $45^{\circ}$ in the forward direction with respect to the beam axis. The Faraday cup is located at a distance of $1.5 \mathrm{~m}$ outside the detector frame to stop the incident beam.

Particles and $\gamma$ rays produced in the reactions are measured in both particle- $\gamma$ 


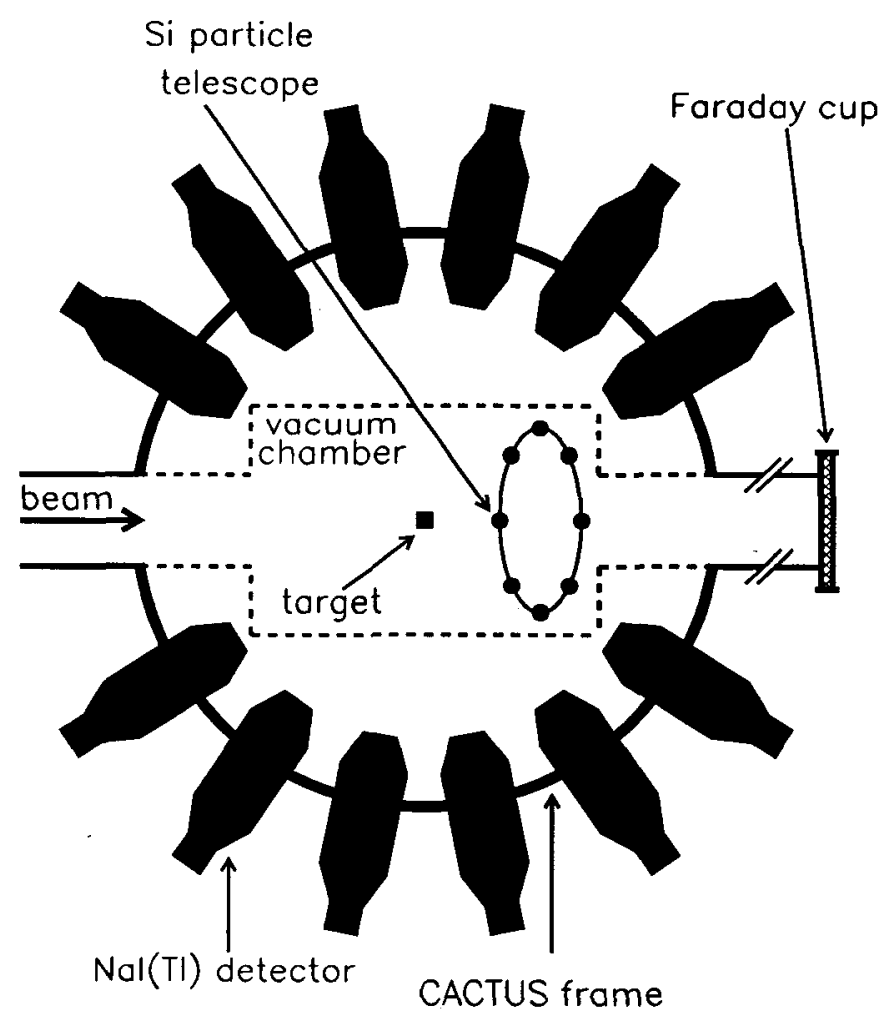

Figure 4.1: Schematic drawing of the CACTUS multidetector array ([56].

Table 4.3: Position of the NaI(Tl) detectors.

\begin{tabular}{ccc|ccc}
\hline \hline Det. \# & $\theta(\mathrm{deg})$ & $\phi(\mathrm{deg})$ & Det. \# & $\theta(\mathrm{deg})$ & $\phi(\mathrm{deg})$ \\
\hline 1 & 37 & 36 & 15 & 101 & 0 \\
2 & 37 & 324 & 16 & 101 & 288 \\
3 & 63 & 216 & 17 & 101 & 216 \\
4 & 37 & 180 & 18 & 101 & 144 \\
5 & 63 & 144 & 19 & 117 & 108 \\
6 & 63 & 72 & 20 & 117 & 36 \\
7 & 79 & 36 & 21 & 117 & 324 \\
8 & 63 & 0 & 22 & 117 & 252 \\
9 & 79 & 324 & 23 & 143 & 216 \\
10 & 63 & 288 & 24 & 117 & 180 \\
11 & 79 & 252 & 25 & 143 & 144 \\
12 & 79 & 180 & 26 & 143 & 72 \\
13 & 79 & 108 & 27 & 143 & 0 \\
14 & 101 & 72 & 28 & 143 & 288 \\
\hline \hline
\end{tabular}


Table 4.4: Position of the 60\% HPGe detector.

\begin{tabular}{cc}
\hline \hline$\theta(\mathrm{deg})$ & $\phi(\mathrm{deg})$ \\
\hline 37 & 108 \\
\hline
\end{tabular}

coincidence and particle singles mode by the CACTUS multi-detector array.

\subsubsection{Silicon Particle Detectors}

Semiconductor detectors, such as silicon and germanium, are essentially solid-state versions of gas-filled ionization chambers. The incident radiation loses energy by an ionization process in which electron-hole pairs are produced, as opposed to electron-ion pairs in the gas-filled chamber. This process occurs in the following way: An electron is raised from the valence band, across the band gap, to the conduction band. If an electric field is present from an external bias, the electron is pulled to the cathode, thus producing a negative charge pulse. The electron that is in the conduction band leaves a hole in the valence band. When the hole is filled by another electron, a new hole is created, which then is filled by another electron, and this process creates a positive charge pulse in the opposite direction of the moving electron. The ideal resolution (FWHM) of a detector is expressed by the formula [57]

$$
\mathrm{FWHM}=2.355 \sqrt{F E \epsilon}
$$

where $E$ is the energy deposited in the detector, $F$ is the so-called Fano factor, which implies deviation from Poisson statistics, and $\epsilon$ is the energy to create a pulse carrier. The average energy to create an electron-hole pair in silicon is $3.81 \mathrm{eV}$, which is 10 times smaller than that required for gas ionization. Furthermore, the Fano factor for semiconductor detectors is less than that for gas or scintillator detectors. Correspondingly the energy resolution is excellent.

As shown in Fig. 4.2, each particle telescope consists of one $\mathrm{Si}$ front and one $\mathrm{Si}(\mathrm{Li})$ 


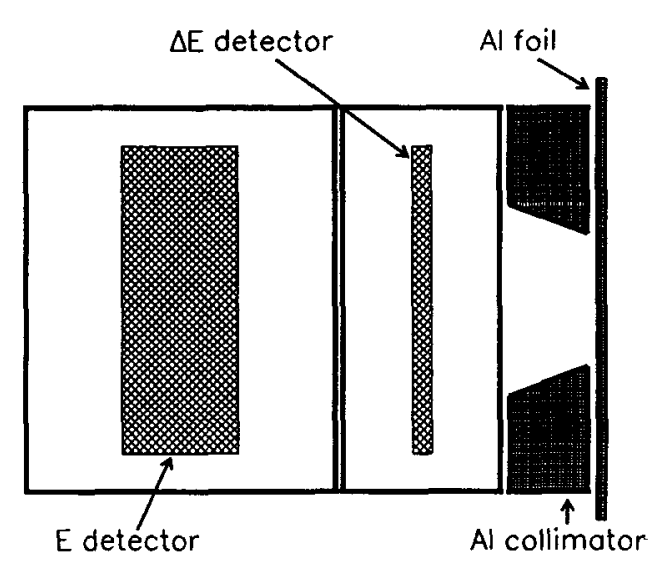

Figure 4.2: Schematic drawing of a Si particle detector.

end detector, respectively called $\triangle \mathrm{E}$ and $\mathrm{E}$. The thicknesses of the $\triangle \mathrm{E}$ and $\mathrm{E}$ detectors are chosen such that particles of interest with maximum energy will be stopped in the end detector. An $\alpha$ particle with $15-\mathrm{MeV}$ energy or more can pass through an $\triangle \mathrm{E}$ detector with a thickness of $150 \mu \mathrm{m}$. The E detectors can stop $\alpha$ particles up to $80 \mathrm{MeV}$. The end detectors have a thickness of $3000 \mu \mathrm{m}$. The thickness of the front detectors is about 145 $\mu \mathrm{m}$, and varies from one detector to another, as listed in Table 4.5. The leakage current $I_{\text {leakage }}$ for each detector is also given in Table 4.5. The leakage current in semiconductor detectors is the current that is created by any source other than ionizing radiation and appears as noise in the detector output pulse. Due to a very high leakage current level, the detector \# 1 was not used for data analysis.

Table 4.5: The thicknesses and leakage currents for the end detectors.

\begin{tabular}{ccc}
\hline Det. \# & $\Delta \mathrm{E}$ thickness $(\mu \mathrm{m})$ & $\mathrm{I}_{\text {leakage }}(\mu \mathrm{A})$ \\
\hline 1 & 144 & $>10$ \\
2 & 150 & 10 \\
3 & 142 & 3 \\
4 & 140 & 3 \\
5 & 137 & 8 \\
6 & 143 & 3 \\
7 & 143 & 7 \\
8 & 140 & 2 \\
\hline
\end{tabular}


As shown in Fig. 4.2, the reaction particles are collimated by a 3-mm thick aluminum collimator with a 3.5-mm radius. When the beam passes through the target a large number of low-energy electrons (so-called $\delta$ electrons) are freed. Because these electrons produce unwanted signals, the front detectors have a $19-\mu \mathrm{m}$ thick $\mathrm{Al}$ foil to stop the $\delta$ electrons. The energy resolution of the silicon detectors is about $400 \mathrm{keV}$ at $40 \mathrm{MeV}$.

In order to distinguish between different types of outgoing particles measured by the silicon detectors, particularly between ${ }^{3} \mathrm{He}$ and $\alpha$ particles, the $\triangle \mathrm{E}-\mathrm{E}$ particledetector technique is employed. This technique uses the fact that the energy loss of a charged particle per unit length in a medium is a function of the charge and the mass of the penetrating particle. The Bethe-Bloch formula is a commonly-used expression for quantum-mechanical calculations of the energy loss

$$
-\frac{d E}{d x}=2 \pi N_{a} r_{e}^{2} m_{e} c^{2} \rho \frac{Z}{A} \frac{z^{2}}{\beta^{2}}\left[\ln \left(\frac{2 m_{e} \gamma^{2} v^{2} W_{\max }}{I^{2}}\right)-2 \beta^{2}\right]
$$

where

$$
\begin{aligned}
& \mathrm{N}_{a}: \text { Avogadro's number }=\quad \mathrm{z} \quad: \text { atomic number of incident } \\
& 6.022 \times 10^{23} \mathrm{~mole}^{-1} \quad \text { particle } \\
& \mathrm{r}_{e}: \text { classical electron } \quad \beta \quad: \quad v / c \text { of the incident particle } \\
& \text { radius }=2.817 \times 10^{-13} \mathrm{~cm} \quad \gamma \quad: 1 / \sqrt{1-\beta^{2}} \\
& \mathrm{~m}_{e}: \text { electron mass } \quad \mathrm{I} \quad \text { : mean excitation potential } \\
& \rho \quad \text { : density of absorbing material } \mathrm{W}_{\max }: \text { maximum energy transfer } \\
& \mathrm{Z} \text { : atomic number of absorbing in a single collision } \\
& \text { material } \\
& \text { A : atomic weight of absorbing } \\
& \text { material }
\end{aligned}
$$

According to Eq. 4.2 the rate of energy loss increases as the charged particle slows down. The penetration depth or range as a function of particle kinetic energy differs for each charged particle due to their different charge and mass numbers. A given particle 
loses a different fraction of its energy in the front and end detectors depending on its energy. Therefore the $\triangle \mathrm{E}$ vs. $\mathrm{E}$ plot shows different patterns for each type of charged particle. Additional discussion on the $\triangle \mathrm{E}-\mathrm{E}$ technique is given in connection with the particle-detector analysis in Chapter 5.

\subsubsection{Gamma-ray Detectors}

In nuclear physics experiments, several types of $\gamma$-ray detectors are used, including highpurity germanium detectors (HPGe), and thallium-activated sodium iodide detectors $(\mathrm{NaI}(\mathrm{Tl}))$. The HPGe detectors are semiconductor detectors, briefly described in the previous section, and have excellent energy resolution. The average energy required to create an electron-hole pair in germanium detectors is only $2.96 \mathrm{eV}$. Thus the amount of ionization produced by incident radiation in the Ge detectors is very large compared to other $\gamma$-ray detectors. Furthermore, the small Fano factor results in increased energy resolution. However, Ge detectors cost much more than do $\mathrm{NaI}(\mathrm{Tl})$ detectors. In addition, all semiconductor detectors, except silicon, require cooling at low temperatures to be operated. As shown in Fig. 4.3, a cryogenic system is attached to a germanium detector. Therefore, the CACTUS multi-detector array is dominated by NaI(Tl) detectors. Only one HPGe detector ( $60 \%$ efficient relative to a $3 "$ x 3 " $\mathrm{NaI}(\mathrm{Tl})$ detector) was used in the present experiment in order to identify the reaction $\gamma$ rays, and therefore the reaction channel.

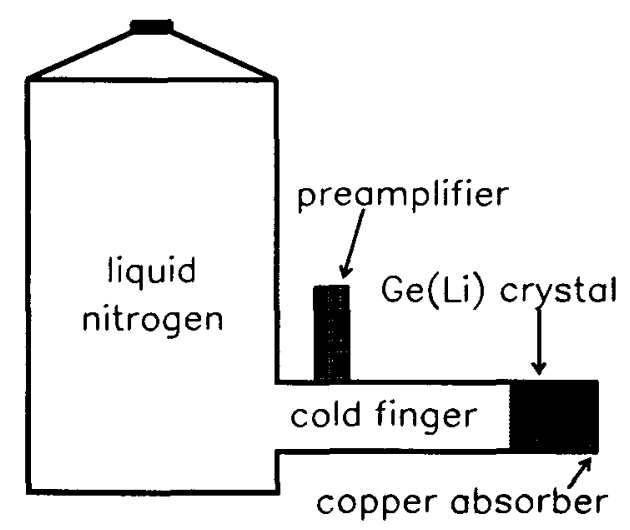

Figure 4.3: Schematic drawing of a Ge detector. 
The $\mathrm{NaI}(\mathrm{Tl})$ detectors are scintillation devices, and are used with photomultiplier (PM) tubes, as shown in Fig. 4.4. When the incident radiation enters the detector, electrons are raised to the conduction band. Eventually the excited electron loses its energy by emitting visible or near-visible light when it recombines with the hole left in the valence band. To increase the probability of the light emission and to reduce self absorption of the light, small amounts of thallium impurities are introduced to the crystal. The impurities provide states in the energy gap, and light emission takes place between these states. When the light emitted by the crystal strikes the photosensitive surface of the PM tube, it releases one or more photoelectrons. These electrons are then multiplied, accelerated, and formed into an output pulse in the PM tube. The shape of the pulse carries time information about the collection of the incident $\gamma$ ray, while the magnitude of the pulse is proportional to the energy of the radiation deposited in the $\mathrm{NaI}(\mathrm{Tl})$ crystal. Large volume $\mathrm{NaI}(\mathrm{Tl})$ detectors provide a very high detection efficiency. However, several factors in the process of formation of the electronic pulse in the PM tube, such as nonradiative recombination and fluctuations in the amplification of the photoelectrons, result in poor energy resolution in the $\mathrm{NaI}(\mathrm{Tl})$ detectors.

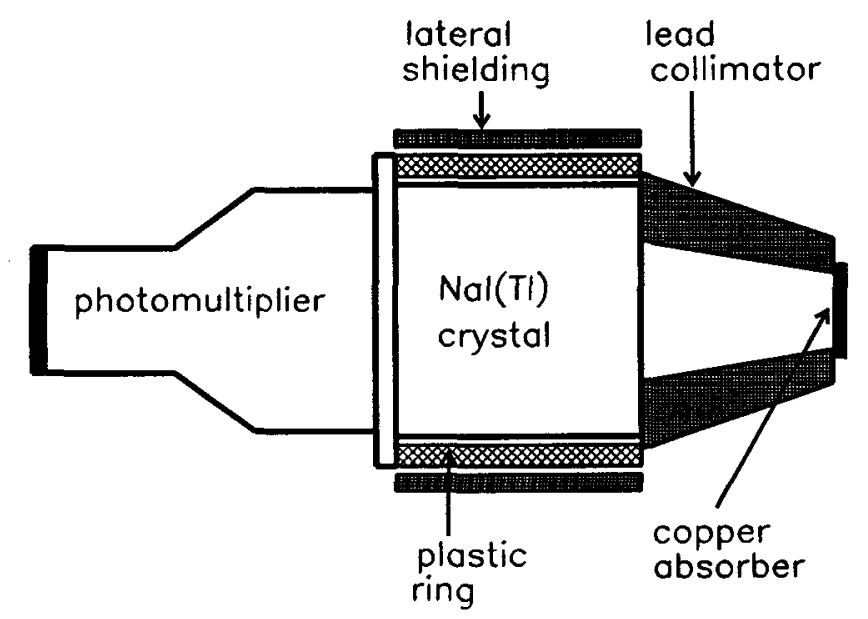

Figure 4.4: Schematic drawing of a NaI(Tl) detector.

The $\gamma$ rays produced during the reaction processes are collimated with lead collimators. In order to absorb the $X$ rays produced by the interaction of the $\gamma$ rays with lead, a 2-mm thick copper absorber is placed in front of the collimator. The NaI(Tl) detectors are 
surrounded by a plastic ring for neutron shielding. One disadvantage of a multidetector array is the crosstalk between the detectors. This occurs when a $\gamma$ ray interacts with one of the detectors and then scatters into another detector. In this case, a $\gamma$ ray is measured by at least two detectors within the detector response time. A 3-mm thick lateral shielding is used to reduce the crosstalk between the detectors.

\subsection{Electronics}

The electronics at the Oslo Cyclotron Laboratory are located in two places, one in the experimental room and the other in the control room. Figure 4.5 shows the electronics diagram in the experimental room where the time signals from the particle and Ge detectors are obtained and transfered to the setup in the control room.

The pulses from the particle and Ge detectors are first preamplified and then sent to timing filter amplifiers (TFA) to produce time signals. In the case of the end detectors, the detector output signals are first summed by a linear fan (Lin OR) in a group of four, designated by $\mathrm{E}_{A}$ and $\mathrm{E}_{B}$ in Fig. 4.5, and then sent to the TFAs since there are not enough modules to process each end detector signal separately. The TFA outputs are processed by constant fraction discriminators (CFD) to generate timing logic signals. The CFD signals of the front detectors are connected to a multiplicity logic unit $(\mathrm{N}=1)$. This unit gives a signal only if one front detector is hit. The output signal is then tested with a pile-up rejection module (PUR) in order to detect the pile-up events, i.e., the PUR module simply takes the logic signal from the multiplicity unit, and stretches it for $\approx 1$ $\mu \mathrm{s}$; if another pulse arrives within the time interval, it gives an inverted logic signal as an indication of a pile-up event. Then the pile-up signal is sent to a VME trigger pattern unit (TPU) where the event is cancelled to prevent further processing. The number of pile-up events was also monitored with a scaler during the experiment.

A summed attenuated analog output of the particle front detector CFD is amplified by a TFA for a threshold setting $(\mathrm{Z}>1$ ). Because fast electrons and $\mathrm{H}$ isotopes deposit only small amounts of energy in the front detector, they are ruled out with this threshold setting at an early stage of the data acquisition. A delayed output from the $N=1$ unit is 


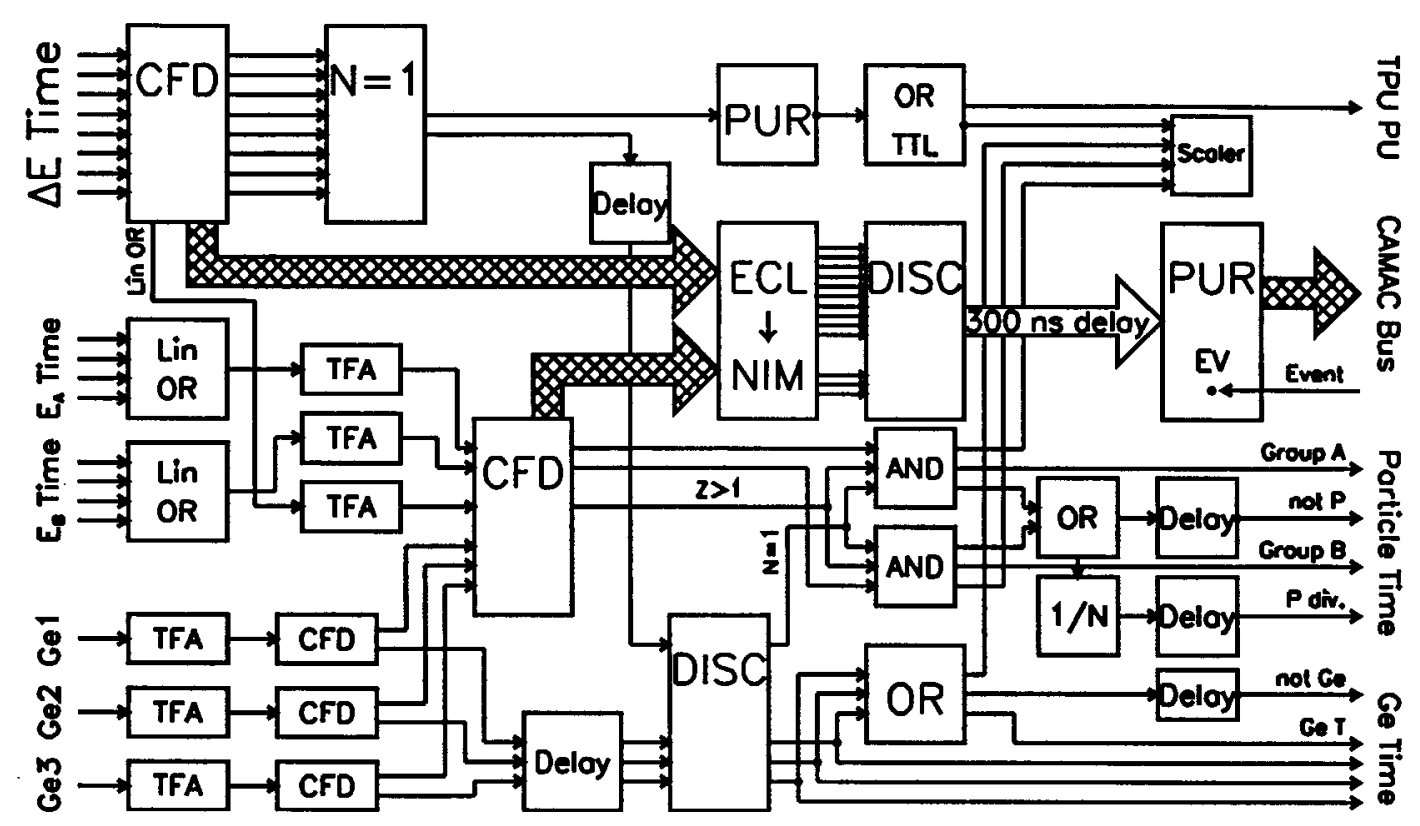

Figure 4.5: Diagram of electronics in the experimental room. The figure is taken from Ref. [58].

sent to a coincidence unit (AND) in order to produce the coincidence between the front and end detectors.

The end detector CFD logic signal, a delayed front-detector $(\mathrm{N}=1)$ unit signal, and the $\mathrm{Z}>1$ CFD signal are connected to a coincidence unit (AND) to test whether all three signals are in coincidence. In other words, the coincidence unit requires three conditions: i) only one front detector is hit $(\mathrm{N}=1)$, ii) the particle front detector CFD signal is higher than a threshold setting $(Z>1)$, iii) at least one particle end detector is hit. If all three conditions are satisfied then the coincidence unit gives a signal. Unfortunately, there is no requirement that the particle front and end detectors of the same telescope are hit, and that only one particle end detector is hit. Because the particle multiplicity and the beam current are relatively low, this is not considered a serious problem. A scaler serves as a counter for several unit signals, such as the coincidence rate of the particle detectors and the summed rate of the Ge detectors. Since the elastically-scattered ${ }^{3} \mathrm{He}$ particles have a 
high rate, every other event is rejected with a $(1 / \mathrm{N}$ with $\mathrm{N}=2)$ module unless a $\gamma$-ray detector is hit (shown as $\mathrm{P}$ div. output in Fig. 4.5).

The electronics setup in the control room is shown in Fig. 4.6. Pulses from the $\mathrm{NaI}(\mathrm{Tl})$ detectors are processed by CFDs. One branch from the CFD is first sent to a veto discriminator, where particle- $\gamma$ coincidences are tested. If there is a coincidence, a logic signal is used to start a time-to-digital converter (TDC). Another delayed branch from the $\mathrm{NaI}(\mathrm{Tl}) \mathrm{CFD}$ is used to stop the TDC. The time interval between start and stop signals in the TDC is then digitized to obtain the time information about the event. The gate generators (GG) connected to the discriminator create a gate for the ADC where the energy of the radiation is recorded from the amplifier output pulses that arrive for an analog-to-digital conversion. The other logic units connected to the discriminator are used i) to find which particle-detector group is in coincidence with the $\gamma$ ray, and subsequently gate on the particle detector $\mathrm{ADC}$, ii) to start the Ge detector TAC (the ADC connected to the TAC together works as a TDC unit), iii) to set flags in the VME trigger pattern units. The control of the NIM-ADCs by VME-ADC controllers, and the control of the CAMAC crate by a CAMAC-branch driver are not shown in Fig. 4.6. The VME crate accommodates connections to the SUN-Sparc station via a BIT3 computer, where tape storage on EXABYTE tapes can be performed.

The flag or the hit pattern at each TPU is displayed in Table 4.6. Each flag simply tells if the corresponding detector has the data information. Having the flags in hand, an event matrix is generated by an offline program, as shown in Table 4.7. The particle energy and $\gamma$-ray energy deposited in the germanium detector, and its time information are registered in the TPU block 1 . The $\gamma$-ray energy deposited in the $\mathrm{NaI}(\mathrm{Tl})$ detectors and corresponding time information are recorded in TPU block 2 and TPU block 3. The virtual TPU 4 is not a real module, but is created by the offline program in order to show in which detectors the pile-up events occurred. The event matrix generated by the offline program is used during the data sorting. First the bit test of the trigger pattern unit is checked, and if it is TRUE then the available data information is extracted from the event matrix in Table 4.7. 


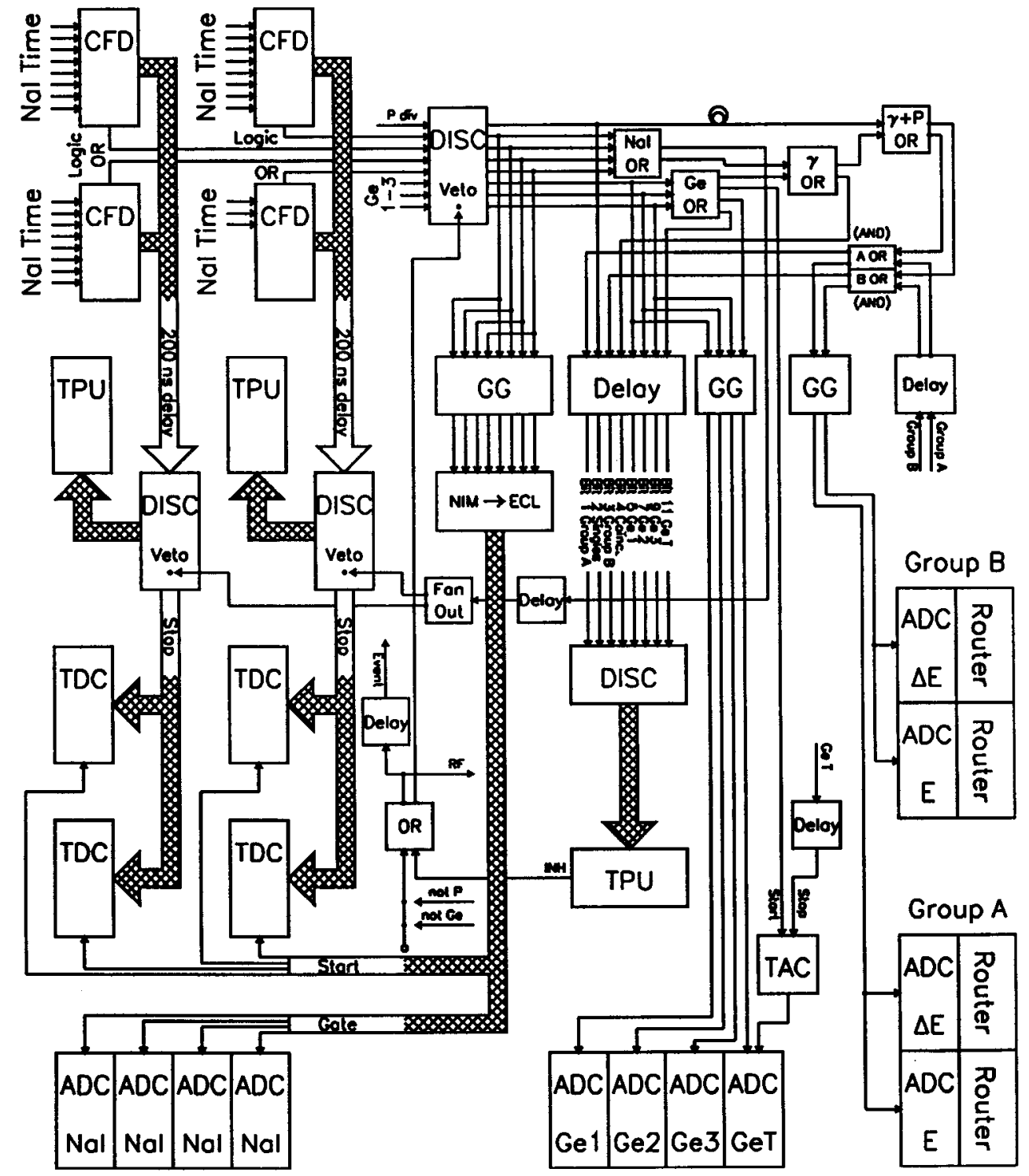

Figure 4.6: Diagram of electronics in the control room. The figure is taken from Ref. [58]. 
Table 4.6: Flags or hit patterns for the TPUs.

\begin{tabular}{|c|c|c|c|c|}
\hline $\begin{array}{l}\text { Bit } \\
\text { number }\end{array}$ & TPU 1 & TPU 2 & TPU 3 & $\begin{array}{l}\text { virtual TPU } 4 \\
\text { (pile up) }\end{array}$ \\
\hline 0 & group A & NaI 1 & NaI 17 & $\triangle \mathrm{E} 1$ \\
\hline 1 & singles & $\mathrm{NaI} 2$ & NaI 18 & $\triangle \mathrm{E} 2$ \\
\hline 2 & group B & NaI 3 & NaI 19 & $\triangle \mathrm{E} 3$ \\
\hline 3 & coincidence & $\mathrm{NaI} 4$ & NaI 20 & $\triangle \mathrm{E} 4$ \\
\hline 4 & Ge 1 & NaI 5 & NaI 21 & $\triangle \mathrm{E} 5$ \\
\hline 5 & & NaI 6 & NaI 22 & $\triangle \mathrm{E} 6$ \\
\hline 6 & Ge 2 & NaI 7 & NaI 23 & $\triangle \mathrm{E} 7$ \\
\hline 7 & & $\mathrm{NaI} 8$ & NaI 24 & $\triangle \mathrm{E} 8$ \\
\hline 8 & Ge 3 & NaI 9 & $\mathrm{NaI} 25$ & Ge 1 \\
\hline 9 & & NaI 10 & NaI 26 & Ge 2 \\
\hline 10 & Ge time & NaI 11 & NaI 27 & Ge 3 \\
\hline 11 & & $\mathrm{NaI} 12$ & NaI 28 & \\
\hline 12 & & $\mathrm{NaI} 13$ & NaI 29 & \\
\hline 13 & & NaI 14 & $\mathrm{NaI} 30$ & \\
\hline 14 & & NaI 15 & NaI 31 & $E(A)$ \\
\hline 15 & & NaI 16 & $\mathrm{NaI} 32$ & $E(B)$ \\
\hline
\end{tabular}

Table 4.7: Event matrix generated by an offline program.

\begin{tabular}{|c|c|c|c|c|c|c|c|c|c|c|c|}
\hline \multirow{2}{*}{$\begin{array}{l}\text { Data } \\
\text { word \# }\end{array}$} & \multicolumn{3}{|c|}{ TPU block 1} & \multicolumn{3}{|c|}{ TPU block 2} & \multicolumn{3}{|c|}{ TPU block 3} & \multicolumn{2}{|c|}{ TPU 4} \\
\hline & 0 & 1 & 2 & 3 & 4 & 5 & 6 & 7 & 8 & 9 & 10 \\
\hline 0 & $0 / 1$ & $\triangle \mathrm{E}(\mathrm{A})$ & $\mathrm{E}(\mathrm{A})$ & $0 / 1$ & NaI1 & time & $0 / 1$ & NaI17 & time & $0 / 1$ & \\
\hline 1 & flags & & & flags & NaI2 & time & flags & NaI18 & time & & flags \\
\hline 2 & & $\triangle \mathrm{E}(\mathrm{B})$ & $E(B)$ & & $\mathrm{NaI} 3$ & time & & NaI19 & time & & \\
\hline 3 & & & & & $\mathrm{NaI} 4$ & time & & NaI20 & time & & \\
\hline 4 & & Ge 1 & & & $\mathrm{NaI} 5$ & time & & NaI21 & time & & \\
\hline 5 & & & & & NaI6 & time & & NaI22 & time & & \\
\hline 6 & & Ge 2 & & & NaI7 & time & & NaI23 & time & & \\
\hline 7 & & & & & NaI8 & time & & $\mathrm{NaI} 24$ & time & & \\
\hline 8 & & Ge 3 & & & $\mathrm{NaI9}$ & time & & $\mathrm{NaI} 25$ & time & & \\
\hline 9 & & & & & NaI10 & time & & $\mathrm{NaI} 26$ & time & & \\
\hline 10 & & Ge time & & & NaI11 & time & & NaI27 & time & & \\
\hline 11 & & & & & $\mathrm{NaI12}$ & time & & $\mathrm{NaI} 28$ & time & & \\
\hline 12 & & & & & NaI13 & time & & NaI29 & time & & \\
\hline 13 & & & & & $\mathrm{NaI14}$ & time & & NaI30 & time & & \\
\hline 14 & & & & & NaI15 & time & & NaI31 & time & & \\
\hline 15 & & & & & NaI16 & time & & NaI32 & time & & \\
\hline
\end{tabular}




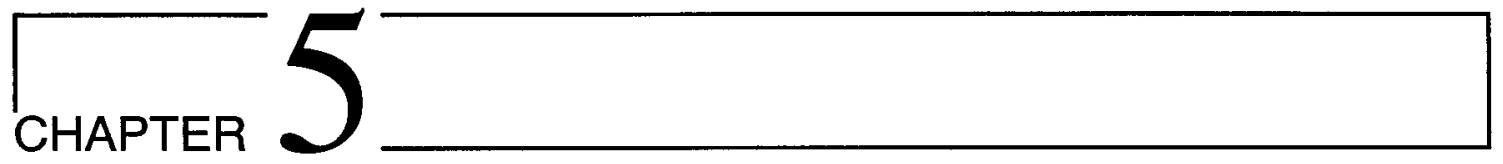

\section{Data Analysis}

The data analysis involves several steps, and each step requires different information extracted from the raw data, such as the particle energy, $\gamma$-ray energy, particle- $\gamma$ ray coincidence matrix, etc. The recorded data have a specific pattern. Several sorting codes written in FORTRAN and C programming languages are used to translate the format of the data written on the tape into a format that the MAMA matrix manipulation program [59], which will be used throughout the analysis, can read. The ultimate goal of the analysis procedure is to obtain a primary $\gamma$-ray spectrum for each excitation energy window in the residual nucleus which is populated in the reaction processes, i.e., an $\mathrm{E}_{x}$ vs. $\mathrm{E}_{\gamma}$ matrix. This matrix will then be used to extract the level densities and $\gamma$-ray strength functions in ${ }^{56} \mathrm{Fe}$ and ${ }^{57} \mathrm{Fe}$. The details of the analysis, including the procedure for unfolding the continuum $\gamma$-ray spectrum and for extracting the primary $\gamma$-ray spectrum, are given in the following sections in this chapter. Some details relating to the mechanism of the ${ }^{57} \mathrm{Fe}\left({ }^{3} \mathrm{He}, \alpha\right){ }^{56} \mathrm{Fe}$ reaction will be useful in the discussion of the analysis.

\subsection{The ${ }^{57} \mathrm{Fe}\left({ }^{3} \mathrm{He}, \alpha \gamma\right){ }^{56} \mathrm{Fe}$ Reaction Mechanism}

Bombardment of the ${ }^{57} \mathrm{Fe}$ target with a $45-\mathrm{MeV}{ }^{3} \mathrm{He}$ beam opens a number of reaction channels, such as ${ }^{57} \mathrm{Fe}\left({ }^{3} \mathrm{He},{ }^{3} \mathrm{He}^{\prime} \gamma\right),{ }^{57} \mathrm{Fe}\left({ }^{3} \mathrm{He}, \alpha \gamma\right)$, and ${ }^{57} \mathrm{Fe}\left({ }^{3} \mathrm{He}, x n \alpha \gamma\right)$, where $x$ is the multiplicity of the neutron emission. Of all of the $\alpha$-out channels the $\left({ }^{3} \mathrm{He}, \alpha \gamma\right)$ reaction is of interest for this discussion. The $\left({ }^{3} \mathrm{He}, \alpha \gamma\right)$ reaction is a direct neutron transfer reaction 
which transforms the target nucleus to the isotope with one less neutron in its ground or an excited state. Once the outgoing $\alpha$-particle energy is measured, one can determine the excitation energy of the residual nucleus ${ }^{56} \mathrm{Fe}$ from the reaction kinematics.

The reaction $Q$ value is defined as the initial mass energy minus final mass energy; thus for the ${ }^{57} \mathrm{Fe}\left({ }^{3} \mathrm{He}, \alpha\right){ }^{56} \mathrm{Fe}$ reaction

$$
\begin{aligned}
Q & =\left(m_{3^{H e}}+m_{57} \mathrm{Fe}-m_{\alpha}-m_{56} \mathrm{Fe}\right. \\
& ) c^{2} \\
& =(3.016029+56.935399-4.002603-55.934942) u c^{2} \\
& =0.013883 u c^{2} \\
& =12.931931 \mathrm{MeV}
\end{aligned}
$$

where $1 u c^{2}=931.494013 \mathrm{MeV}$. The positive $Q$ value indicates that energy is released as kinetic energy or as excitation energy of the final products.

The spin and parity of the incident particle and the target nucleus are $s=1 / 2^{+}$and $I=1 / 2^{-}$, respectively. The total angular momentum $J$ is a combination of three angular momenta: the orbital angular momentum $l$, the spin $s$ of the incident particle, and the spin $I$ of the target (all in units of $\hbar$ ). The channel spin is defined as $S=s+I$. In this case, $S$ can be 0 or 1 . If the incident particle is in its $l$ th partial wave, the total angular momentum $J$ assumes the values $|l-S|, \ldots,|l+S|$. Given that the kinetic energy of the incident particle is $45 \mathrm{MeV}$, the orbital angular momentum can be found approximately from the classical relation of kinetic energy to angular momentum

$$
E=\frac{l(l+1) \hbar^{2}}{2 m a^{2}}
$$

where $a$ is the impact parameter, taken as $a=1.2 A^{1 / 3} \mathrm{fm}$ with $A=3$, and $m$ is the mass of the incident particle. One finds 


$$
l(l+1) \approx 13
$$

which implies $l$ to be 3 or 4 . If $S=0$, then $J=3$ for $l=3$, and $J=4$ for $l=4$. If $S=1$, we find $J=2,3,4$ for $l=3$, while $J=3,4,5$ for $l=4$. If the outgoing $\alpha$ particle does not carry away much of the angular momentum, the residual nucleus is left with most of the angular momentum. The current configuration of the particle telescopes does not allow angular distribution measurements of the $\alpha$ particles since the telescopes are fixed at $45^{\circ}$ forward angle with respect to the beam direction. From the above considerations we can estimate that the $\left({ }^{3} \mathrm{He}, \alpha \gamma\right)$ reaction populates low-spin states with about $2-8 \hbar$ in ${ }^{56} \mathrm{Fe}$.

\subsection{Particle-Spectra Analysis}

As explained in Chapter 4, each silicon particle telescope consists of one front $(\triangle E)$ and one end $(\mathrm{E})$ detector. The raw data are sorted to obtain the spectra of the front and end particle detectors, as shown in Fig. 5.1.

According to the Bethe-Bloch formula (see Eq. 4.2) slow particles deposit a large amount of their energies in the front telescope, while high-energy particles pass through the front telescope with little energy loss, and then deposit the rest of their energy in the end detector. Therefore, the lower channels in the $\triangle E$ spectrum and the higher channels in the $\mathrm{E}$ spectrum correspond to particles with high energy.

The prominent peak in both the $\triangle \mathrm{E}$ and $\mathrm{E}$ spectra in Fig. 5.1 corresponds to elastically-scattered ${ }^{3} \mathrm{He}$ particles. The counts below the elastic peak in the $\triangle \mathrm{E}$ spectrum are due to the $\mathrm{H}$ isotopes, i.e., $\mathrm{p},{ }^{2} \mathrm{H}$, and ${ }^{3} \mathrm{H}$. The counts above the elastic peak are due to $\alpha$ particles from the $\left({ }^{3} \mathrm{He}, x n \alpha\right)$ reaction channels, and ${ }^{3} \mathrm{He}$ particles from the inelastic scattering channel. Similarly, lower channels below the elastic peak in the E spectrum are due to the inelastically scattered ${ }^{3} \mathrm{He}$ particles, the $\mathrm{H}$ isotopes, and slow $\alpha$ particles from the $\left({ }^{3} \mathrm{He}, x n \alpha\right)$ reactions. Because the ${ }^{57} \mathrm{Fe}\left({ }^{3} \mathrm{He}, \alpha\right)$ reaction has a positive $Q$ value, the $\alpha$ particles from this reaction appear above the elastic peak. The peak structures in this 

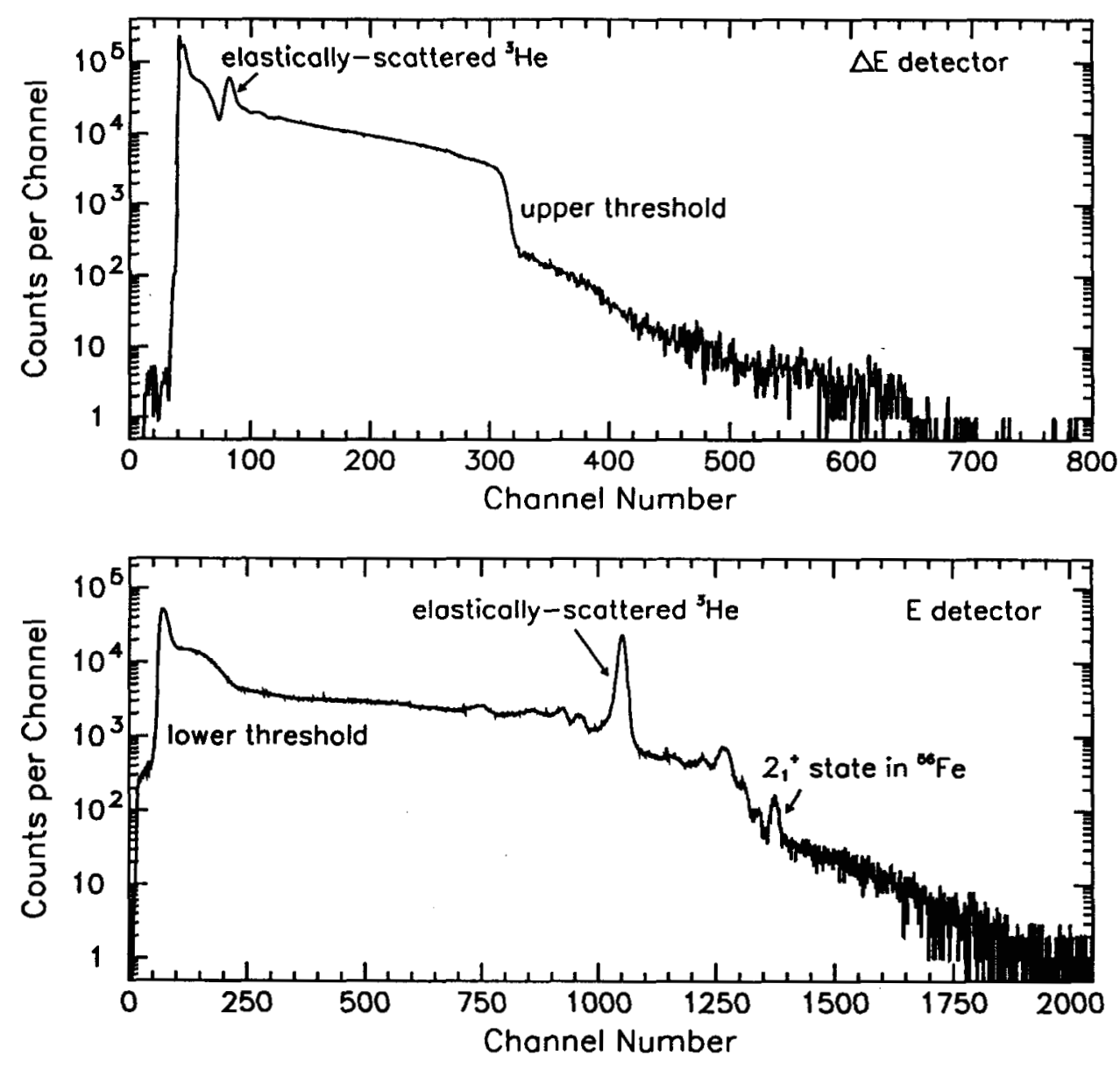

Figure 5.1: Particle spectra obtained from the front and end particle detector 3 .

region are the discrete levels in ${ }^{56} \mathrm{Fe}$. The counts above the channel of the ground state are due to detector pile-up events. These events occur when two particles are detected in the same detector; then the energy is the sum of the two energies, resulting in a higher channel.

In order to identify the ${ }^{56} \mathrm{Fe}$ discrete levels in the $\mathrm{E}$ spectrum, this spectrum is compared with the particle spectrum from Ref. [60], which was obtained from a similar ${ }^{57} \mathrm{Fe}\left({ }^{3} \mathrm{He}, \alpha\right)$ experiment with a $14.3-\mathrm{MeV}$ incident beam and $33^{\circ}$ scattering angle. The $2_{1}^{+}$ state in ${ }^{56} \mathrm{Fe}$ is identified by this comparison and is shown in Fig. 5.1. With two peaks 
identified, the elastic and the $2_{1}^{+}$-state peak, one can easily determine the gain and shift for the end detectors and calibrate the E spectrum.

First one needs to determine the energy deposited in the E detector for these peaks. Particles lose energy due to straggling in the target. This energy is calculated for the ${ }^{3} \mathrm{He}$ and $\alpha$ particles. Then the energy loss in the Al foil is determined from the BetheBloch formula (see Eq. 4.2). Next, the energy loss in the front detectors for the elastic ${ }^{3} \mathrm{He}$ particles and for the $\alpha$ particles, which leave the residual ${ }^{56} \mathrm{Fe}$ in the first-excited state, were determined using the Bethe-Bloch formula. This calculation gives the energies of these ejectiles that were deposited in the end detectors. The end-detector spectrum is calibrated using these energies and the centroid channels for the ${ }^{3} \mathrm{He}$ and $\alpha$ particles. Similarly, we need at least two calibration points to calibrate the front detectors. Since the events in the upper end of the $\triangle E$ spectrum are essentially the same events as those in the lower threshold of the $\mathrm{E}$ spectrum, one can use the upper threshold of the $\triangle \mathrm{E}$ spectrum as a calibration point. Its energy is determined by applying the E-spectrum calibration to the energy of the lower threshold. Then the energy loss for $\alpha$ particles in the front telescope is calculated from the Bethe-Bloch formula. Finally, the gain and shift for the $\triangle \mathrm{E}$ detector are found using the energy loss for elastically-scattered ${ }^{3} \mathrm{He}$ particles and the threshold events.

\subsection{Data Reduction}

Using the gain and shift obtained for the particle detectors as an input, the small drifts in the calibration of the end detectors are calculated with a program called REDUC [61]. The REDUC code accumulates data of 20 records, determines the position of the elasticallyscattered ${ }^{3} \mathrm{He}$ peak in the end detector spectrum, calculates a correction factor for the calibration of the end-detector spectra (assuming that the ADC shifts of these spectra are constant), and writes it onto a new tape. Furthermore, the code REDUC rejects the events that are associated with proton- or deuteron-out channels by setting a broad gate in their thickness spectra, which will be described in the next section. Therefore, the particle spectra are aligned and are mostly free of protons and deuterons on the new tape. 


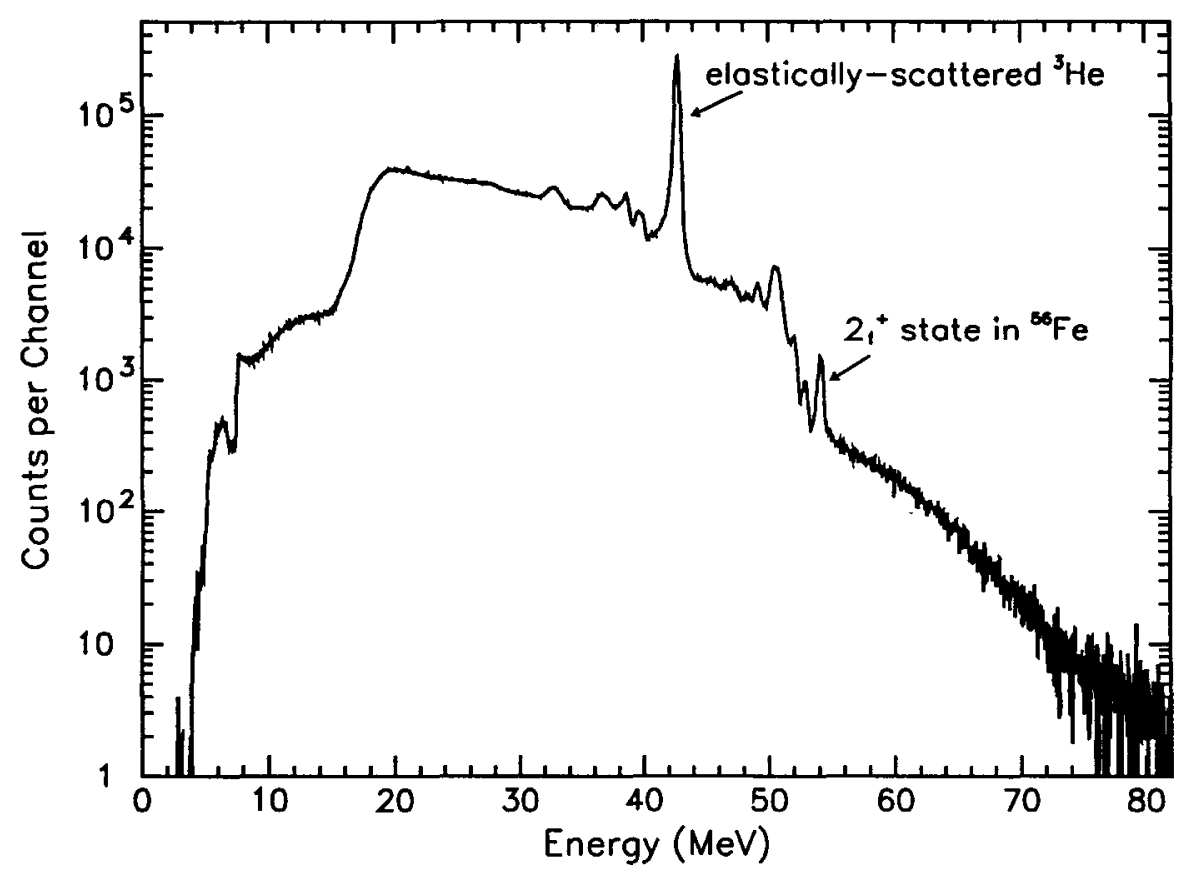

Figure 5.2: Total particle energy spectrum.

The particle spectrum on the new tape has a resolution of $40 \mathrm{keV}$ per channel.

Once the particle front and end spectra are all aligned, one obtains the total energy of a measured particle by adding the energy deposited in the front and end detectors. Figure 5.2 is obtained by summing the front and end energies from all of the telescopes.

\subsection{Gating on $\alpha$ Particles}

The reduced data are sorted into a two-dimensional matrix with the $\triangle \mathrm{E}$ energy along one axis and the $E$ energy along the other axis. The characteristics of a $\triangle E$ vs. E spectrum is that for each type of particle there is a unique banana-shaped curve. In Fig. 5.3, an example of a $\triangle \mathrm{E}-\mathrm{E}$ spectrum is shown; one can readily distinguish between the ${ }^{3} \mathrm{He}$ and $\alpha$ particles. The $\alpha$ banana includes $\alpha$ particles from reaction channels that eject an $\alpha$ particle, i.e., $\left({ }^{3} \mathrm{He}, \alpha\right)$ and $\left({ }^{3} \mathrm{He}, x n \alpha\right)$, where $x$ is the multiplicity of the emitted neutrons. By projecting the matrix onto the $\triangle \mathrm{E}(\mathrm{E})$ axis, one obtains a front-detector (end-detector) spectrum. By projecting the matrix along the opposite diagonal with $\mathrm{E}+\Delta \mathrm{E}=\mathrm{E}_{\text {tot }}$, one 
obtains the total energy spectrum. The $2_{1}^{+}$-state peak from the single $\alpha$-out channel and the elastic ${ }^{3} \mathrm{He}$ peak can be found at the right end of the respective banana curves. The tail leading away from the elastic peak is too asymmetric to be explained by straggling in the front detector. A more likely explanation is that a small amount of the ejectiles are channeling through the front detector due to its polycrystalline structure. The $\mathrm{H}$ isotopes are not completely eliminated from the spectrum in the process of reducing the data, as seen below the ${ }^{3} \mathrm{He}$ banana in Fig. 5.3.

In order to gate on a specific particle the $\triangle \mathrm{E}-\mathrm{E}$ technique introduced in Chapter 4 will be used. First we need to construct a range curve. A range curve simply describes how deep a certain particle with a given kinetic energy can penetrate a certain material. In general, for a charged particle this is dominated by energy loss due to ionization. Earlier experiments with the CACTUS multidetector array showed that the range curve can be described accurately with the following parameterization [58]:

$$
R(E)=a E^{(b+c \log E)}
$$

where $a, b$, and $c$ are free parameters, and $E$ is the kinetic energy of the charged particle. From the definition of the range curve, it follows that the total range for a given particle minus the range for that particle in the end detector equals the thickness of the front detector:

$$
R(\triangle E+E)-R(E)=d_{\text {front }},
$$

where $\triangle E$ and $E$ are the energies deposited in the front and end detector, respectively. We would like to obtain the parameters $a, b$, and $c$. First, the energies deposited in the E and $\triangle E$ detectors for several events are obtained from the $\triangle \mathrm{E}-\mathrm{E}$ matrix (Fig. 5.3). The points marked along the $\alpha$-banana curve are selected for this purpose. Then Eq. 5.5 is solved for the marked $\triangle \mathrm{E}$ and $\mathrm{E}$ energies by fitting the parameters of Eq. 5.4. The fit 


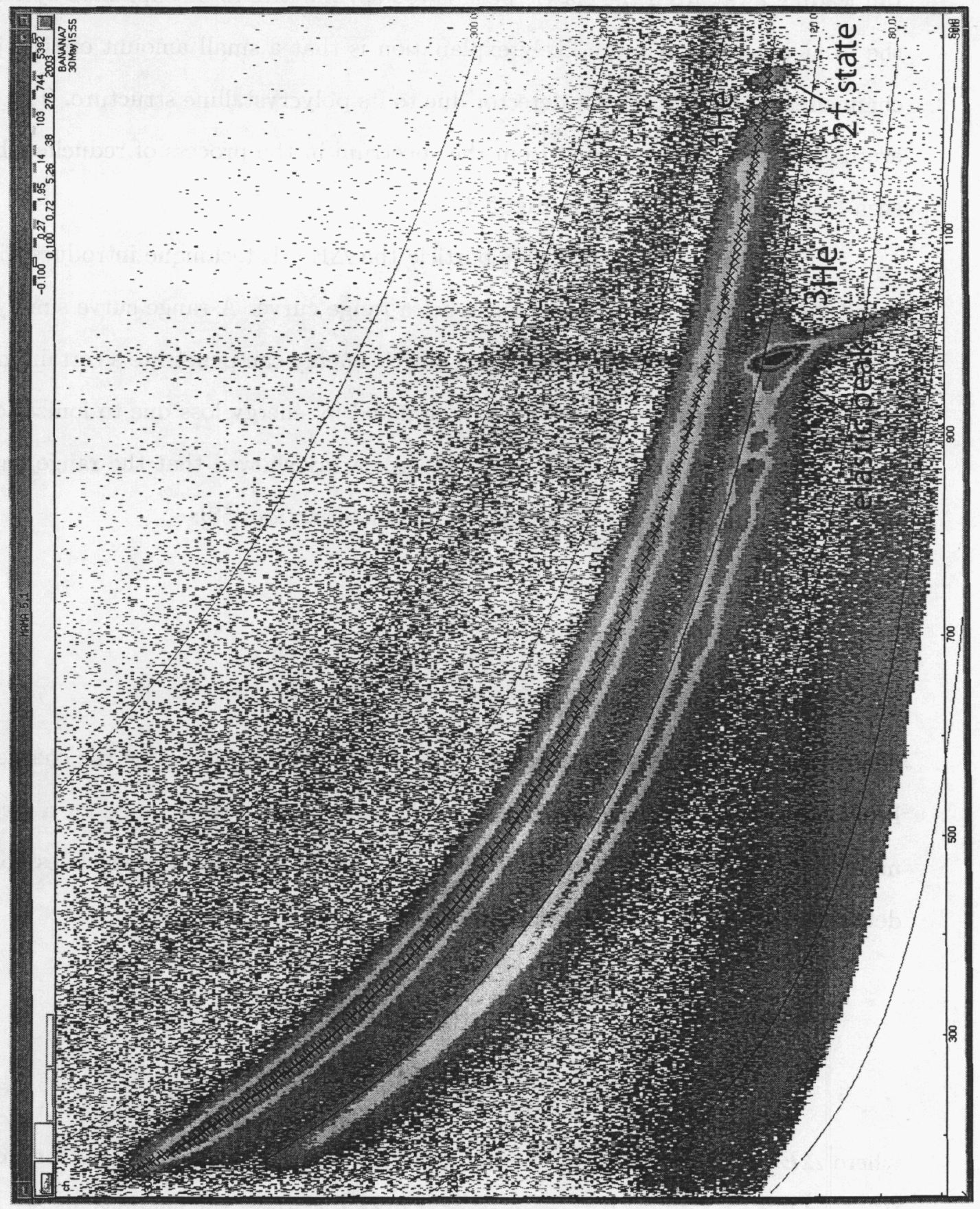

Figure 5.3: $\triangle \mathrm{E}$ vs. E spectrum obtained from telescope 7. 


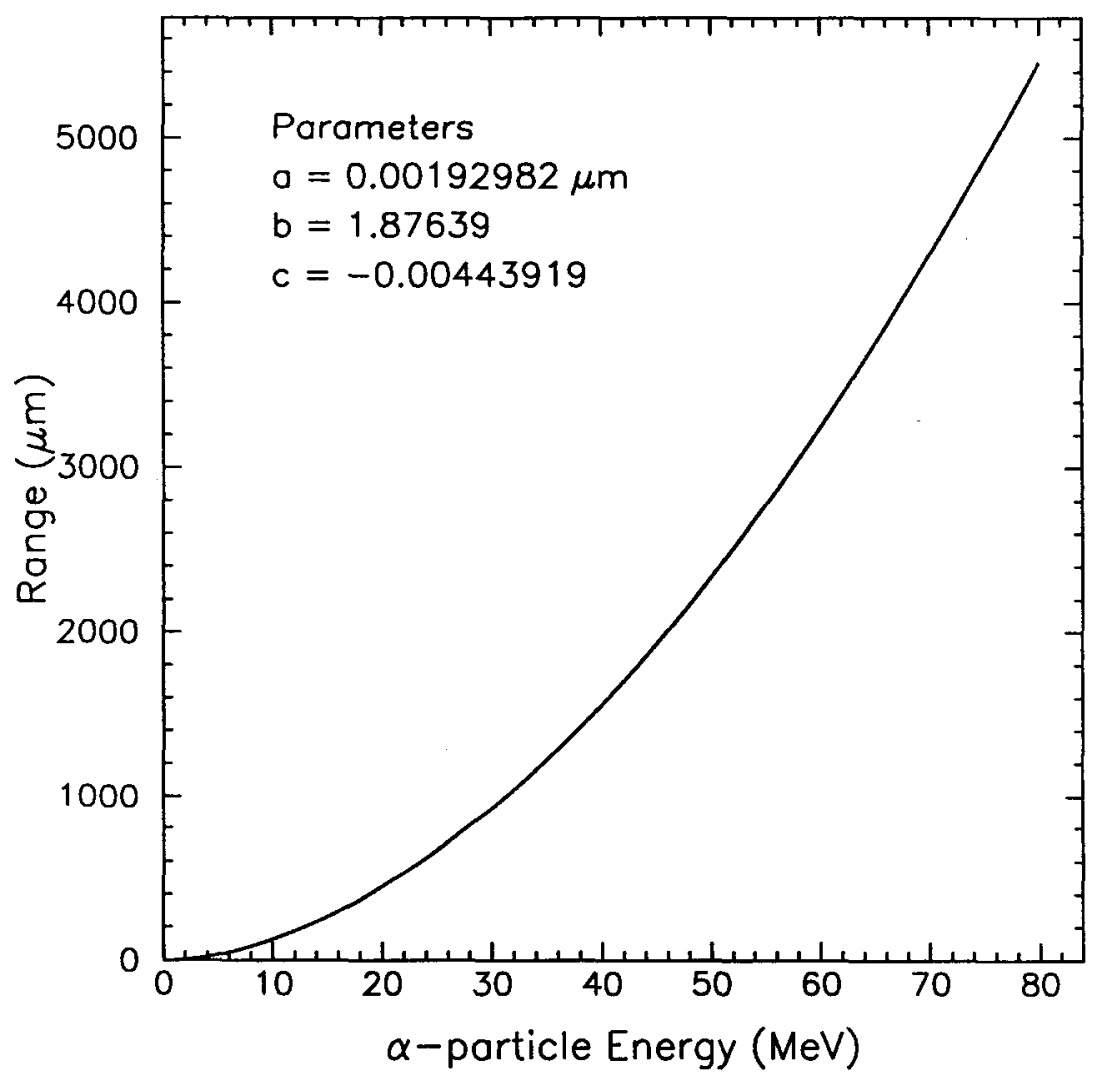

Figure 5.4: Range curve for $\alpha$ particles in silicon.

displayed in Fig. 5.3 as a solid line describes the $\alpha$ banana very well. The other parallel lines correspond to the fits for different thicknesses of the front detector. The resulting range curve for $\alpha$ particles in silicon is plotted in Fig. 5.4.

Once the parameters $a, b$, and $c$ are determined, the range curve is employed in the next data sorting, and by inserting the particle energies into Eq. 5.5, $d_{\text {front }}$ is found for every particle event. The new spectrum obtained this way is referred to as the thickness spectrum. The thickness spectrum is essentially the projection of the data in the $\triangle \mathrm{E}-\mathrm{E}$ matrix along the solid lines.

Figure 5.5 shows the thickness spectrum obtained for telescope 7. A thickness spectrum is obtained for each particle telescope. The peaks for the ${ }^{3} \mathrm{He}$ and $\alpha$ particles are well separated. The peak centroid for the $\alpha$ particles agrees with the thickness of 


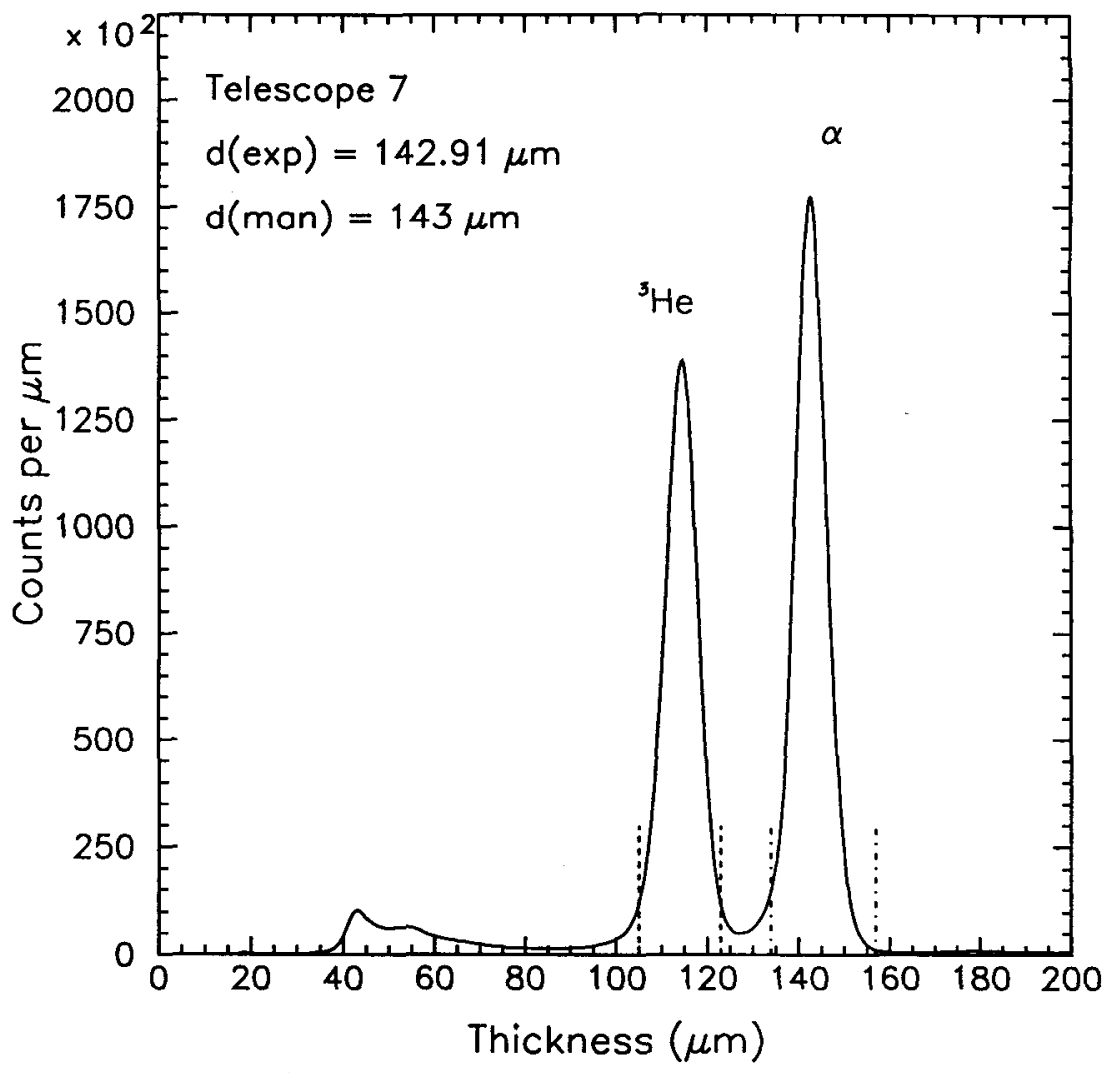

Figure 5.5: Thickness spectrum of particle telescope 7.

the $\triangle \mathrm{E}$ detectors from the specification sheets delivered by the manufacturers. Finally, one can set a gate for the ${ }^{3} \mathrm{He}$ and $\alpha$ particles in the thickness spectrum, as shown by the dashed lines in Fig. 5.5. These gates will be used in subsequent data sort routines to extract the $\alpha-\gamma$ and ${ }^{3} \mathrm{He}-\gamma$ coincidences.

\subsection{Particle $-\gamma$-ray Coincidence Timing}

In the present experiment timing information is needed to determine the particle- $\gamma$ coincidences, i.e., zero timing interval. The best timing information is obtained when the charge signal is collected very rapidly in the detector, and a large number of carriers (ion pairs, electron-hole pairs) are generated by the incident radiation. The time information is registered by a time-to-digital converter (a TAC and an ADC connected together) that 
records the time interval between the input start and stop pulses arriving at the TDC. The distribution of the digitized time intervals between the start and stop pulses is called a time spectrum.

The data are sorted to obtain the timing of the particle-detector signals with respect to the $\gamma$-ray detector signals by gating on the $\alpha$ particles. Given that there are seven particle detectors and $28 \mathrm{NaI}(\mathrm{Tl})$ detectors, one obtains $196 \mathrm{Si}-\mathrm{NaI}(\mathrm{Tl})$ time spectra. The time spectra must be aligned and summed together in order to set a gate in the time spectrum for the particle $-\gamma$-ray coincidences. The time spectra are aligned using a PAW macro [62], assuming two different offsets in the time spectra, one from the particle telescopes, and the other from the $\mathrm{NaI}(\mathrm{Tl})$ detectors. First we determine the centroid positions of the time spectra. These centroids includes the contributions mentioned above, and can be written explicitly in a matrix form

$$
T=\left(\begin{array}{ccccc}
s_{1}+n_{1}+\triangle_{11} & s_{1}+n_{2}+\triangle_{12} & s_{1}+n_{3}+\triangle_{13} & \ldots & s_{1}+n_{28}+\triangle_{128} \\
s_{2}+n_{1}+\triangle_{21} & s_{2}+n_{2}+\triangle_{22} & s_{2}+n_{3}+\triangle_{23} & \ldots & s_{2}+n_{28}+\triangle_{228} \\
\vdots & \vdots & \vdots & & \vdots \\
s_{7}+n_{1}+\triangle_{71} & s_{7}+n_{2}+\triangle_{72} & s_{7}+n_{3}+\triangle_{73} & \ldots & s_{7}+n_{28}+\triangle_{728}
\end{array}\right)
$$

where $s$ and $n$ are the contributions from the $\mathrm{Si}(\mathrm{Li})$ and $\mathrm{NaI}(\mathrm{Tl})$ detectors, respectively, and $\triangle_{i j}$ are the fluctuations from these components. Alternatively, one could write

$$
T_{i j}=s_{i}+n_{j}+\triangle_{i j}
$$

The total sum in each matrix element is already known. We would like to find the individual components $s_{i}$ and $n_{j}$. This can be done in the following steps: 
1. $\bar{S}_{i}=s_{i}+\bar{n}+\bar{\triangle}_{i}$

each row is added, and divided by the number of $\mathrm{NaI}(\mathrm{Tl})$ detectors

2. $\quad \bar{N}_{j}=\bar{s}+n_{j}+\bar{\triangle}_{j}$ each column is added, and divided by the number of particle detectors

3. $\frac{\sum_{i=1}^{7} \bar{S}_{i}}{7}=\frac{\sum_{j=1}^{28} \bar{N}_{j}}{28}=\bar{s}+\bar{n} \quad \bar{\triangle}_{i}$ and $\bar{\triangle}_{j}$ are assumed to be zero due to averaging

4. $\bar{s}+\bar{n}+\mathrm{x}=300$ the centroid channel for the aligned spectrum will be at channel 300

5. $\mathrm{x}=300-\bar{n}$ $\bar{s}$ is set to zero, $\mathrm{x}$ is the shift due to drift in the time spectrum

In the above procedure, the fluctuations in the $s_{i}$ and $n_{j}$ components are averaged. Therefore, it is safe to assume that they are zero. We cannot find the $\bar{s}$ and $\bar{n}$ separately. Therefore, we set $\bar{s}$ to zero, and calculate the individual $s_{i}$ and $n_{j}$ from the first and second steps. The new aligned time spectrum is displayed in Fig. 5.6. The time spectrum is fitted by the function [58]

$$
y=\frac{a}{b+\left|x_{0}-x\right|^{\nu \pm \mu}} \quad \text { where }\left\{\begin{array}{l}
+ \text { if } x_{0} \geq x \\
- \text { if } x_{0}<x
\end{array}\right.
$$

where $x_{0}$ is the channel of the centroid, and $a, b, \nu$, and $\mu$ are parameters to be determined. The maximum of the function is $y_{\max }=a / b$ at $x_{0}=x$. If the channels on two sides of the centroid at the half maximum are $x_{1}$ and $x_{2}$, one can find the FWHM by calculating $\left(x_{0}-x_{1}\right)+\left(x_{2}-x_{0}\right)$, i.e.,

$$
\begin{aligned}
& \frac{y_{\max }}{2}=\frac{a}{b+\left(x_{0}-x_{1}\right)^{\nu+\mu}} \\
& \frac{y_{\max }}{2}=\frac{a}{b+\left(x_{2}-x_{0}\right)^{\nu-\mu}},
\end{aligned}
$$


by using $\mathrm{y}_{\max }=a / b$ in Eq 5.7 one obtains

$$
\begin{aligned}
& x_{0}-x_{1}=b^{\frac{1}{\nu+\mu}} \\
& x_{2}-x_{0}=b^{\frac{1}{\nu-\mu}} .
\end{aligned}
$$

Then the FWHM is

$$
F W H M=b^{\frac{1}{\nu+\mu}}+b^{\frac{1}{\nu-\mu}} .
$$

The fit is performed between channels 200 and 440 assuming a linear background. The FWHM is found to be $\approx 40$ channels. The gain of the time spectrum is $0.5 \mathrm{~ns} /$ channel.

The background subtracted area of the prominent peak in Fig. 5.6 gives the true + chance coincidences. The area of the peak to the left of the prominent peak gives the chance coincidences with a previous beam pulse. The gates 1 and 2 shown with dashed lines in Fig. 5.6 will be used to obtain the particle- $\gamma$ coincidences. For example, if an event falls into the gate 2 , it will be added; if it falls into the gate 1 , the event will be subtracted, and everything else is rejected.

\subsection{NaI(Tl)-spectra Analysis}

The $\mathrm{NaI}(\mathrm{Tl})$ detectors are calibrated using one in-beam and two background $\gamma$ rays. Unfortunately, these $\gamma$ rays cover a small energy range up to $2614 \mathrm{keV}$ in the $\mathrm{E}_{\gamma}$ spectrum, while the maximum energy is around $10 \mathrm{MeV}$. The lowest $\gamma$-ray energy is $847 \mathrm{keV}$, which is the in-beam $2^{+} \rightarrow 0^{+}$transition in ${ }^{56} \mathrm{Fe}$. A background run performed before the experiment provides two calibration energies, one at $1461 \mathrm{keV}$ (in the ${ }^{40} \mathrm{Ar}$ nucleus), and the other at $2614 \mathrm{keV}$ (in the ${ }^{208} \mathrm{~Pb}$ nucleus). The former results from naturally-occurring

${ }^{40} \mathrm{~K}$ decaying into the first excited state in ${ }^{40} \mathrm{Ar}$ after capturing an electron. The latter is a result of the radioactive thorium-232 series reaching the stable ${ }^{208} \mathrm{~Pb}$ nucleus.

The centroid channels corresponding to the three $\gamma$-ray energies are fitted linearly 


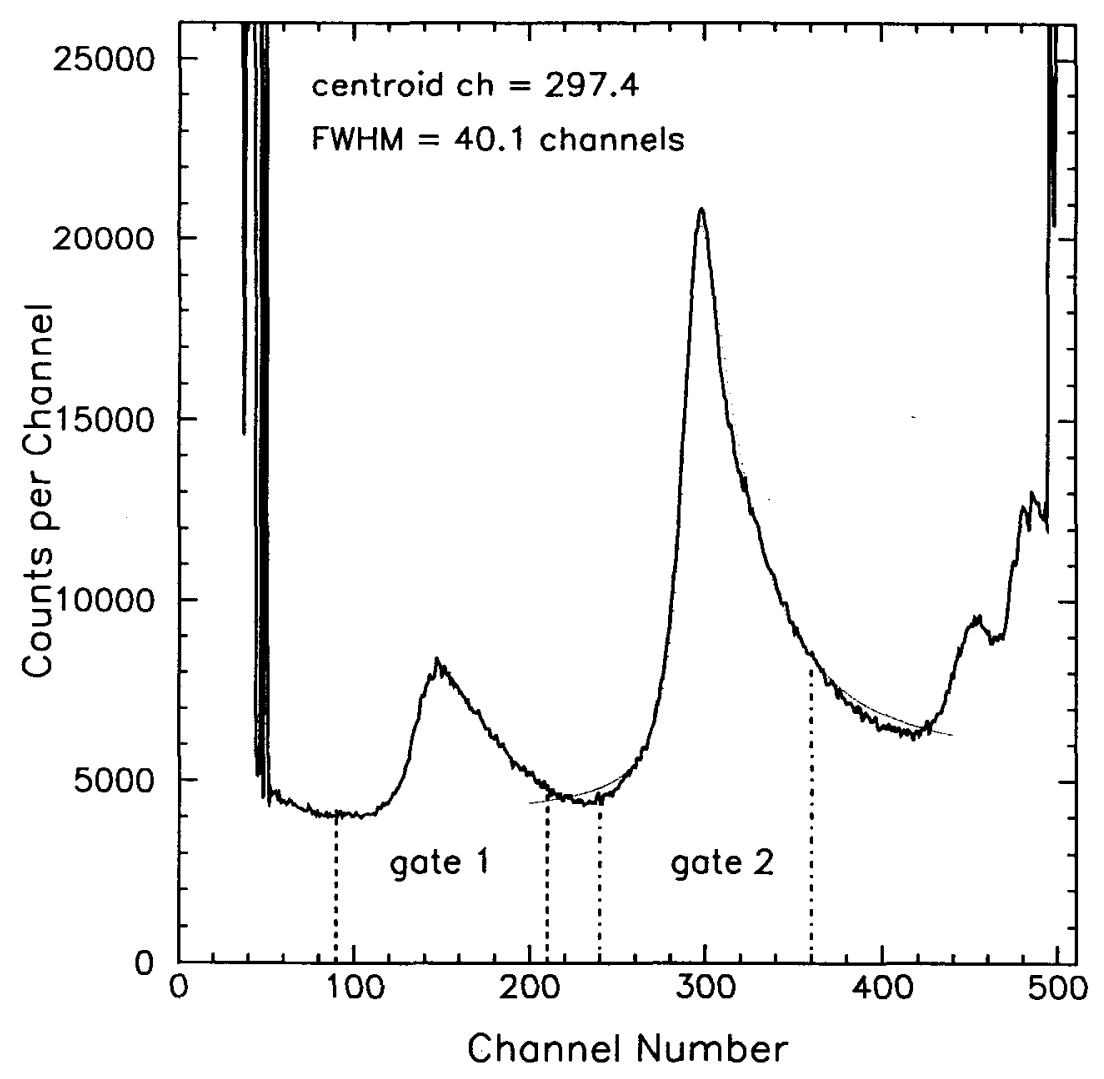

Figure 5.6: Time spectrum of the particle detectors with respect to the $\mathrm{NaI}(\mathrm{TI})$ detectors.

for each $\mathrm{NaI}(\mathrm{Tl})$ detector. Figure 5.7 shows the residuals of the linear fit, obtained by subtracting the experimental channels from the channels calculated from the linear fit. The parameters of the linear fit are also displayed in Fig. 5.7. For a good calibration all of the residual data points should be close to zero. For the majority of the detectors the residual data points lie between zero and four channels in Fig. 5.7. The difference between the experimental and the calculated channels reaches about ten channels for a few detectors. Since the detector gain is $10 \mathrm{keV}$ per channel, ten channels correspond to a $100 \mathrm{keV}$ difference. A further approach to refine this calibration would be fitting the residuals by a nonlinear function. In our case, however, this procedure is dangerous because we do not have calibration points to define the behavior of the nonlinear function 

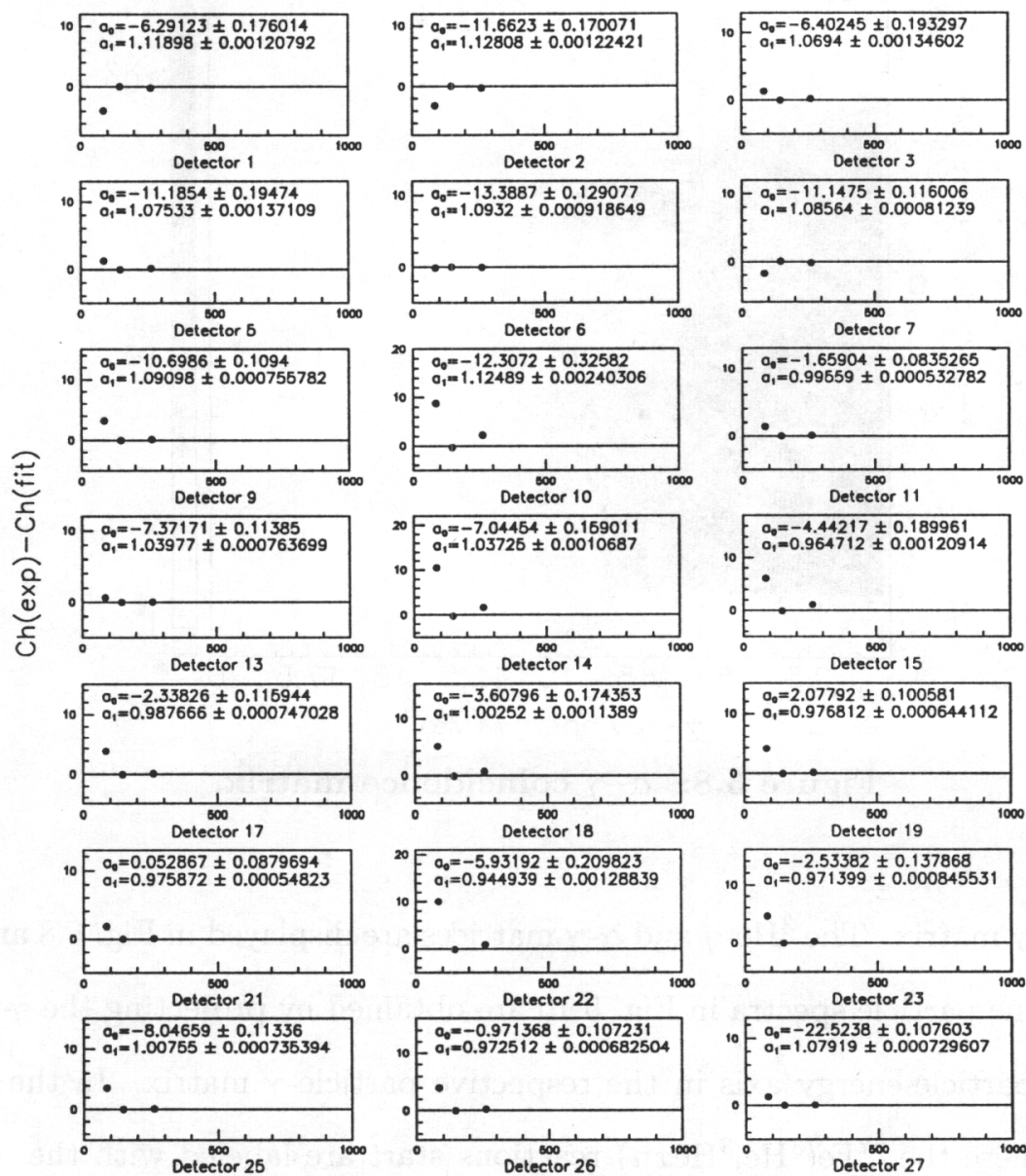

Channel(new $)=E(k e V) /(10 \mathrm{keV} / \mathrm{ch})$
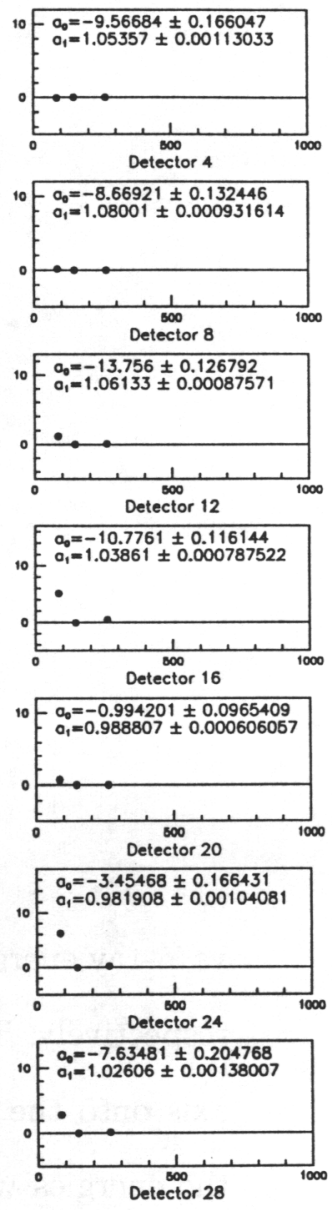

Figure 5.7: Energy calibration for the NaI(Tl)-detectors.

at high $\gamma$-ray energies. Therefore, a nonlinear fit was not performed.

\subsection{Particle - $\gamma$-ray Matrix}

Thus far the data from the particle detectors and the $\gamma$-ray detectors have been analyzed. The appropriate gates, one on the thickness spectra to gate on a specific particle ( $\alpha$ or ${ }^{3} \mathrm{He}$ ) and the other on the time spectra to gate on the particle $-\gamma$-ray coincidences, are constructed. These gates are used to sort the data into a two-dimensional particle energy 


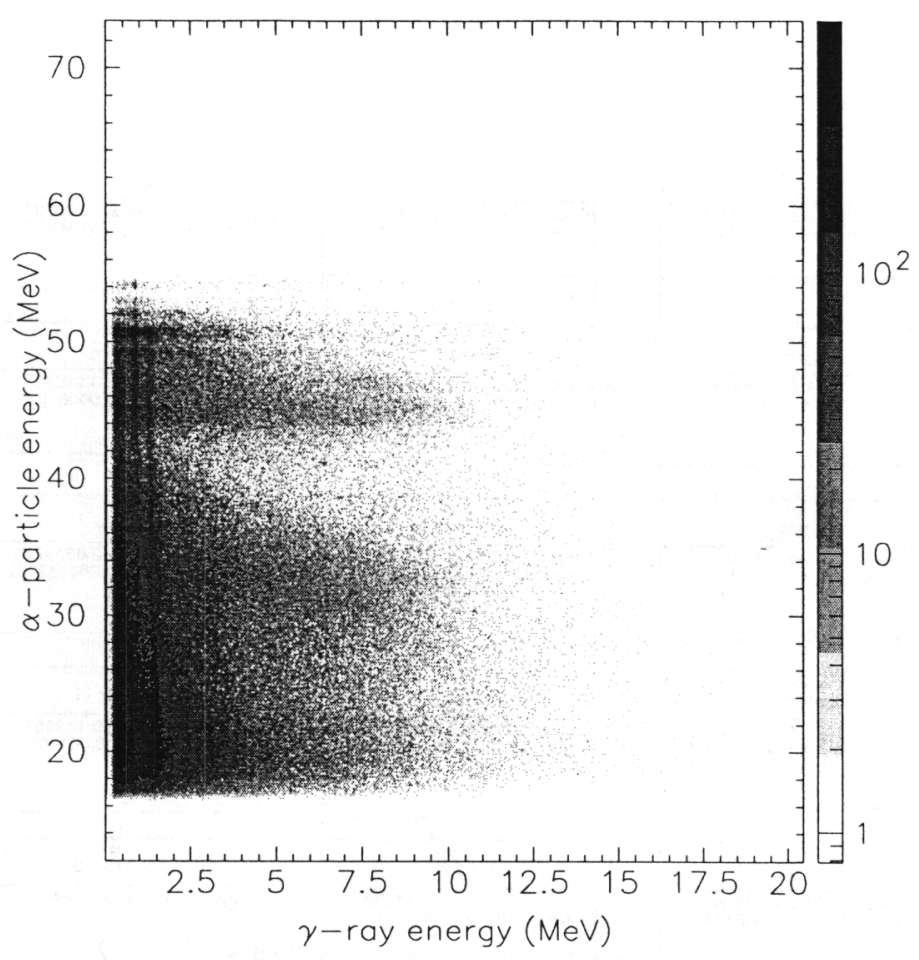

Figure 5.8: $\alpha-\gamma$ coincidence matrix.

vs. $\gamma$-ray energy matrix. The ${ }^{3} \mathrm{He}-\gamma$ and $\alpha-\gamma$ matrices are displayed in Fig. 5.8 and Fig. 5.9, respectively. The particle spectra in Fig. 5.10 are obtained by projecting the $\gamma$-ray energy axis onto the particle-energy axis in the respective particle- $\gamma$ matrix. In the top panel, the energies where the ${ }^{57} \mathrm{Fe}\left({ }^{3} \mathrm{He},{ }^{3} \mathrm{He} x n\right)$ reactions start are labeled with the multiplicity of the neutron emission: e.g., $\mathrm{B}_{2 n}$ corresponds to the ${ }^{57} \mathrm{Fe}\left({ }^{3} \mathrm{He}, \alpha 2 n\right){ }^{54} \mathrm{Fe}$ channel. The most energetic $\alpha$ particles are those from the $\left({ }^{3} \mathrm{He}, \alpha\right)$ reaction channel. When neutrons are emitted from the compound nucleus along with the $\alpha$ particles, the energy is shared between the neutrons and the $\alpha$ particles; therefore, those $\alpha$ particles appear in the lowenergy region in Fig. 5.10. Similarly, the energies where the ${ }^{57} \mathrm{Fe}\left({ }^{3} \mathrm{He},{ }^{3} \mathrm{He} x n\right)$ reactions start to dominate are labeled in the lower panel in Fig. 5.10. The maximum ${ }^{3} \mathrm{He}$-particle energy is around $43 \mathrm{MeV}$, a few $\mathrm{MeV}$ less than the initial $45 \mathrm{MeV}$ energy, due to the energy loss in the target and the $\mathrm{Al}$ foil in front of the detectors. The difference in the maximum energies for the ${ }^{3} \mathrm{He}$ and $\alpha$ particles in the two spectra results from the positive $Q$ value of the $\left({ }^{3} \mathrm{He}, \alpha\right)$ reaction. 


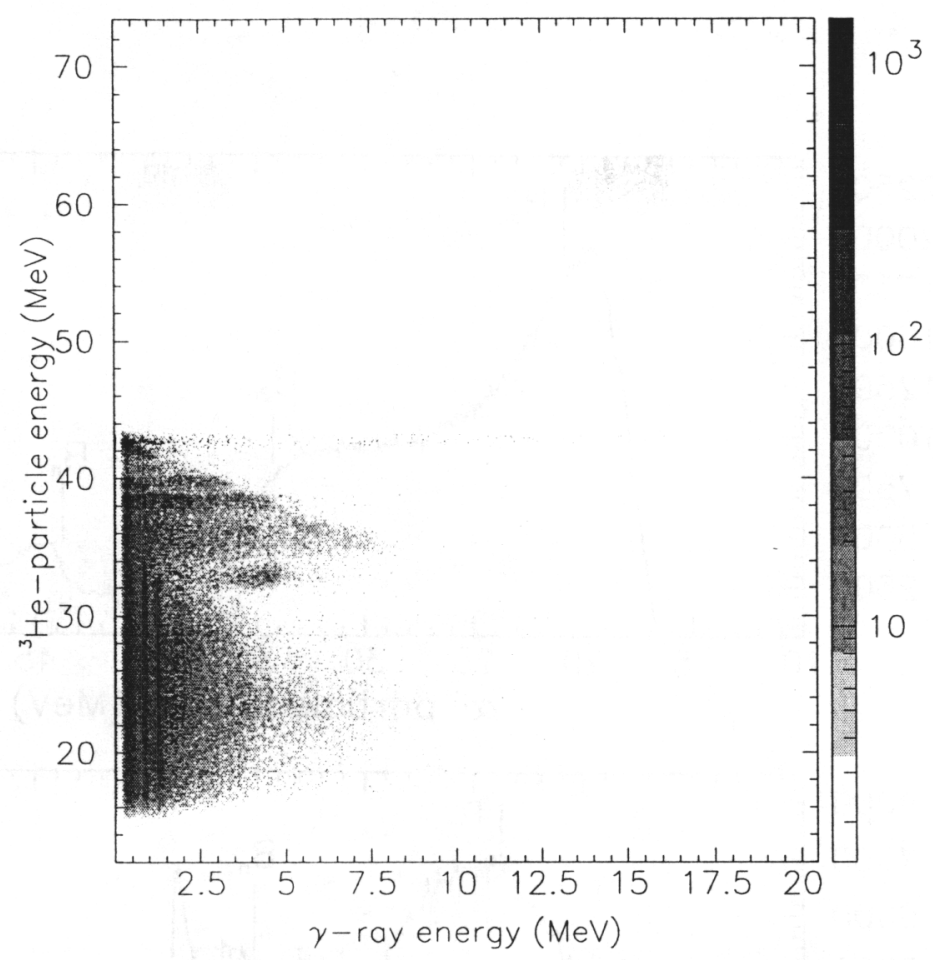

Figure 5.9: ${ }^{3} \mathrm{He}-\gamma$ coincidence matrix.

For the following analysis procedure, the particle energy is transformed into the excitation energy of the product nucleus. Note that the particle detectors were already calibrated using the reaction kinematics; thus that calibration is employed for the energy transformation. Since we are interested in the $\left({ }^{3} \mathrm{He}, \alpha\right)$ and $\left({ }^{3} \mathrm{He}^{3}{ }^{3} \mathrm{He}^{\prime}\right)$ reaction channels, we take a partition in the corresponding particle vs. $\gamma$-ray energy matrix only for those reaction channels.

\subsection{Unfolding the Total $\gamma$-ray Spectra}

When an incident $\gamma$ ray hits a detector, it interacts with the detector material in three ways: Compton scattering, photoelectric absorption, and pair production. The $\gamma$ ray can Compton scatter several times; and after each scattering, it loses some energy and a free electron is produced. At low $\gamma$-ray energies photoelectric absorption becomes dominant and the photon vanishes. If the original $\gamma$ ray loses all of its energy in the detector, the deposited energy is equal to the original $\gamma$-ray energy, and the respective peak in the energy 

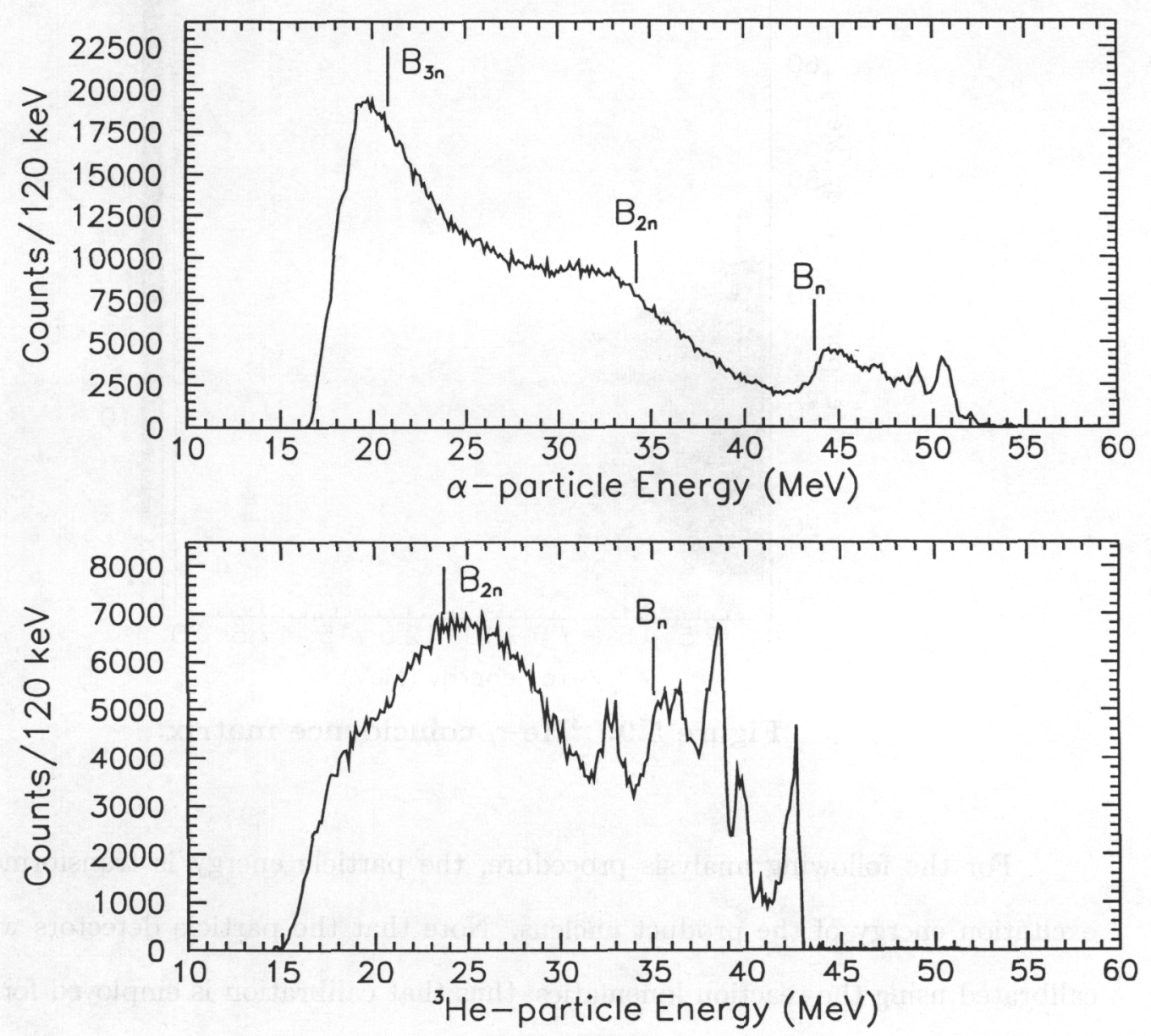

Figure 5.10: Particle spectra gated on $\gamma$ rays.

spectrum is called the full-energy peak or photopeak. Unfortunately, Compton scattered photons may escape from the detector due to finite-detector size. These events appear in the lower energy side of the full-energy peak as a continuum called the Compton continuum. The incident radiation might also lose energy by producing an electron-positron pair. Then the pair production is followed by positron annihilation, Compton scattering, and photoelectric absorption; again complete energy loss occurs. If one of the annihilation photons leaves the detector, the $\gamma$ ray deposits its full energy minus $511 \mathrm{keV}$. If both annihilation photons leave the detector, it results in energy deposition of the full energy minus $1022 \mathrm{keV}$. The corresponding peaks in the energy spectrum are called single-escape 
and double-escape peaks, respectively. The detector response function has contributions from all of these processes. In addition, the $\approx 200-\mathrm{keV}$ backscattering and 511-keV annihilation peaks, which originate in the material surrounding the detector, appear in the detector response function. The response function may depend on several variables, such as operating conditions of the detector and detector geometry, and it is very important to subtract the incomplete-energy contributions from the total $\gamma$-ray spectrum in order to obtain the full-energy events.

Thus it is necessary to know the detector response function for each $\gamma$-ray energy. For $\gamma$ rays with no response function, one needs an appropriate interpolation procedure to find response functions for a large range of $\gamma$-ray energies. In the next section we give details of interpolating the response matrix for the CACTUS multidetector array [63]. Then the $\gamma$-ray spectra will be unfolded using the response matrix [63].

\subsubsection{Response Function}

The response functions are obtained for the 28 collimated and shielded $5 "$ x $5 " \mathrm{NaI}(\mathrm{Tl})$ detectors in the CACTUS multidetector array. The measurements are performed with ten monoenergetic $\gamma$ rays: $122,245,344,662,1173,1333,1836,4439,6130,15110 \mathrm{keV}$. The response functions for other $\gamma$-ray energies are obtained by an interpolation between the known response functions. The interpolation of the Compton background and the prominent peaks (single escape, double escape, annihilation, and back scattering peaks) are performed separately. This separation both simplifies the interpolation procedure, and allows one to construct a new response matrix when the experimental energy resolution is different from the resolution of the observed response matrix. Thus the Compton background (c) is obtained by subtracting the full-energy (f), single-escape (s), doubleescape (d), and annihilation (a) peaks from the observed spectra. Then the probabilities that an event belongs to one of the five components is normalized to one, i.e.,

$$
p_{f}+p_{c}+p_{s}+p_{d}+p_{a}=1
$$


These probabilities, the full-width at half maximum (FWHM), and the total $\gamma$-ray efficiencies are listed in Table 5.1.

The interpolation between the peak structures $(f, s, d, a)$ is performed simply by placing a Gaussian distribution at the interpolated peak position with the proper intensity and energy resolution. The interpolation of the Compton background is more complicated, and relies on the fact that Compton scattering is a slowly-varying function of the incident $\gamma$-ray energy. The energy transferred to an electron or the energy deposited in the detector, in a Compton scattering event is given by

$$
E=E_{\gamma}-\frac{E_{\gamma}}{1+\frac{E_{\gamma}}{m_{0} c^{2}}(1-\cos \theta)}
$$

where $m_{0} c^{2}$ is the rest mass energy of the electron and $\theta$ is the angle of the scattered $\gamma$ ray from the original direction. The Compton backgrounds for different incident $\gamma$-ray energies are shown in Fig. 5.11. The spectra $C_{1}$ and $C_{2}$ in Fig. 5.11 are the measured and normalized Compton spectra, and the spectrum $\mathrm{C}$ is to be obtained by interpolation. The Compton background becomes more compressed with decreasing $\gamma$-ray energy, thus, $\Delta \theta$ decreases. The interpolation is performed between channels that correspond to the same scattering angle $\theta$, with the assumption that only one Compton scattering occurs and that the scattered $\gamma$ ray escapes from the detector. This assumption is reasonable because a $\gamma$ ray scattered more than once is more likely to deposit its complete energy in the detector. The area covered by $\Delta \theta$ in the Compton spectra can be written

$$
\begin{aligned}
A_{1}\left(E_{\gamma_{1}}\right) & =c_{1}\left(E_{1}\right)\left(\frac{d E}{d \theta}\right)_{E_{\gamma_{1}}} \\
A\left(E_{\gamma}\right) & =c(E)\left(\frac{d E}{d \theta}\right)_{E_{\gamma}} \\
A_{2}\left(E_{\gamma_{2}}\right) & =c_{2}\left(E_{2}\right)\left(\frac{d E}{d \theta}\right)_{E_{\gamma_{2}}} .
\end{aligned}
$$

$E_{\gamma}, E_{\gamma_{1}}$, and $E_{\gamma_{2}}$ are the full energies for the spectra $\mathrm{C}, \mathrm{C}_{1}$, and $\mathrm{C}_{2}$, respectively; $E$, 
Table 5.1: The FWHMs, $\gamma$-ray efficiencies, and intensities deduced for a number of the $\mathrm{NaI}(\mathrm{Tl})$ response functions [63].

\begin{tabular}{ccllllll}
\hline \hline $\mathrm{E}_{\gamma}(\mathrm{keV})$ & ${\mathrm{FWHM}(\mathrm{keV})^{a}}^{a} \epsilon^{b}$ & $\mathrm{p}_{f}$ & $\mathrm{p}_{c}$ & $\mathrm{p}_{s}$ & $\mathrm{p}_{d}$ & $\mathrm{p}_{a}$ \\
\hline 400 & 39.8 & 1.000 & 0.5668 & 0.4332 & 0.0000 & 0.0000 & 0.0000 \\
800 & 56.1 & 1.000 & 0.4778 & 0.5222 & 0.0000 & 0.0000 & 0.0000 \\
1200 & 74.3 & 0.988 & 0.3988 & 0.6012 & 0.0000 & 0.0000 & 0.0000 \\
1600 & 90.4 & 0.952 & 0.3503 & 0.6425 & 0.0046 & 0.0000 & 0.0027 \\
2000 & 100.8 & 0.880 & 0.3205 & 0.6623 & 0.0115 & 0.0003 & 0.0054 \\
2400 & 114.0 & 0.871 & 0.3030 & 0.6718 & 0.0180 & 0.0012 & 0.0060 \\
2800 & 125.0 & 0.861 & 0.2851 & 0.6815 & 0.0248 & 0.0021 & 0.0065 \\
3200 & 133.6 & 0.852 & 0.2666 & 0.6916 & 0.0318 & 0.0030 & 0.0070 \\
3600 & 140.0 & 0.843 & 0.2476 & 0.7019 & 0.0390 & 0.0039 & 0.0076 \\
4000 & 144.0 & 0.833 & 0.2280 & 0.7126 & 0.0464 & 0.0048 & 0.0082 \\
4400 & 155.7 & 0.824 & 0.2077 & 0.7236 & 0.0541 & 0.0058 & 0.0088 \\
4800 & 166.9 & 0.815 & 0.2004 & 0.7299 & 0.0543 & 0.0065 & 0.0088 \\
5200 & 177.7 & 0.810 & 0.1944 & 0.7359 & 0.0537 & 0.0072 & 0.0087 \\
5600 & 187.9 & 0.810 & 0.1883 & 0.7419 & 0.0532 & 0.0079 & 0.0087 \\
6000 & 197.7 & 0.810 & 0.1822 & 0.7480 & 0.0526 & 0.0086 & 0.0086 \\
6400 & 206.9 & 0.810 & 0.1759 & 0.7543 & 0.0520 & 0.0093 & 0.0086 \\
6800 & 215.7 & 0.810 & 0.1695 & 0.7606 & 0.0513 & 0.0101 & 0.0085 \\
7200 & 224.0 & 0.811 & 0.1630 & 0.7670 & 0.0507 & 0.0108 & 0.0085 \\
7600 & 231.8 & 0.812 & 0.1564 & 0.7736 & 0.0501 & 0.0116 & 0.0084 \\
8000 & 239.1 & 0.813 & 0.1496 & 0.7803 & 0.0494 & 0.0123 & 0.0083 \\
8400 & 246.0 & 0.815 & 0.1428 & 0.7871 & 0.0488 & 0.0131 & 0.0083 \\
8800 & 252.3 & 0.816 & 0.1358 & 0.7940 & 0.0481 & 0.0139 & 0.0082 \\
9200 & 258.1 & 0.817 & 0.1287 & 0.8010 & 0.0474 & 0.0147 & 0.0081 \\
9600 & 263.5 & 0.819 & 0.1215 & 0.8081 & 0.0467 & 0.0155 & 0.0081 \\
10000 & 268.4 & 0.820 & 0.1142 & 0.8154 & 0.0460 & 0.0164 & 0.0080 \\
10400 & 272.7 & 0.825 & 0.1067 & 0.8228 & 0.0453 & 0.0172 & 0.0079 \\
10800 & 276.6 & 0.830 & 0.0991 & 0.8304 & 0.0446 & 0.0181 & 0.0079 \\
11200 & 280.0 & 0.836 & 0.0913 & 0.8381 & 0.0438 & 0.0190 & 0.0078 \\
\hline \hline
\end{tabular}

a Normalized to $79.9 \mathrm{keV}(6 \%)$ at $1.33 \mathrm{MeV}$.

${ }^{b}$ Normalized to one at $1.33 \mathrm{MeV}$. This efficiency must be multiplied with the discriminator efficiency of the experimental set-up. 


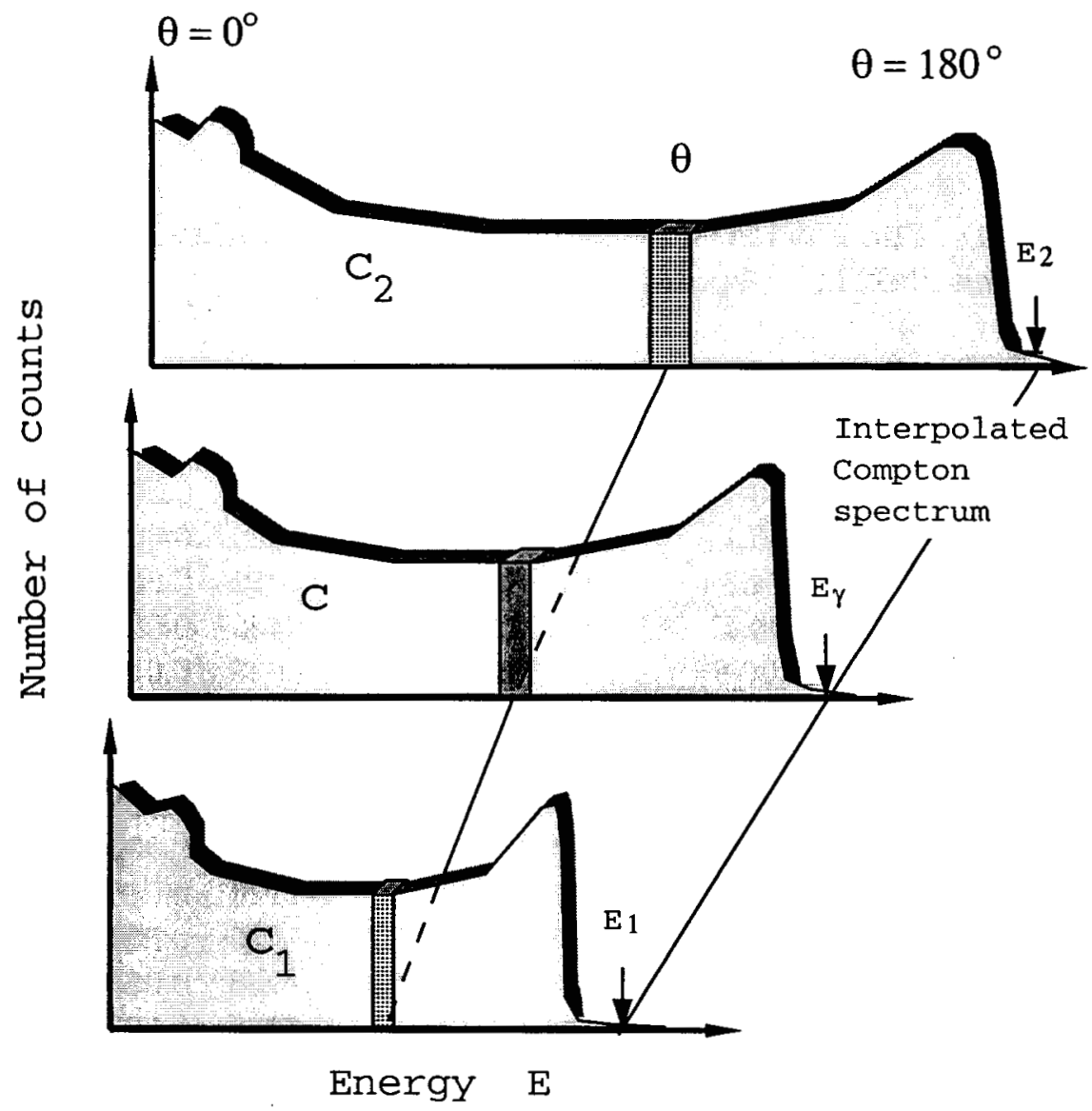

Figure 5.11: Interpolation of Compton background, taken from Ref. [63].

$E_{1}$, and $\mathrm{E}_{2}$ are the corresponding energies deposited in the detector. $c(E), c_{1}\left(E_{1}\right)$, and $c_{2}\left(E_{2}\right)$ are the normalized numbers of counts for the corresponding energy deposited in each spectrum. Assuming that the area $A$ is a linear function of the full energy, i.e., $A=a+b E_{\gamma}$, the coefficients $a$ and $b$ can be obtained using the known $A_{1}$ and $A_{2}$ in Eq. 5.12

$$
\begin{aligned}
a & =A_{1}-\frac{A_{1}-A_{2}}{E_{\gamma_{1}}-E_{\gamma_{2}}} E_{\gamma_{1}} \\
b & =\frac{A_{1}-A_{2}}{E_{\gamma_{1}}-E_{\gamma_{2}}} .
\end{aligned}
$$

The number of counts $c(E)$ for the interpolated energy $E$ is 


$$
c(E)=\left(\frac{d E}{d \theta}\right)_{E_{\gamma}}^{-1} A\left(E_{\gamma}\right)
$$

and if we write $A\left(E_{\gamma}\right)$ explicitly we obtain

$$
c(E)=\left(\frac{d E}{d \theta}\right)_{E_{\gamma}}^{-1}\left[c_{1}\left(E_{1}\right)\left(\frac{d E}{d \theta}\right)_{E_{\gamma_{1}}}+\frac{E_{\gamma}-E_{\gamma_{1}}}{E_{\gamma_{2}}-E_{\gamma_{1}}}\left(c_{2}\left(E_{2}\right)\left(\frac{d E}{d \theta}\right)_{E_{\gamma_{2}}}-c_{1}\left(E_{1}\right)\left(\frac{d E}{d \theta}\right)_{E_{\gamma_{1}}}\right)\right] .
$$

Using Eq. 5.15, one can find the number of counts in the interpolated spectrum. The backscattering peak appears in the lower energy side of the Compton spectrum, and is determined by

$$
E_{b . s c .}=\frac{E_{\gamma}}{1+\frac{2 E_{\gamma}}{m_{e} c^{2}}}
$$

Thus, for very high $\gamma$-ray energies $E_{b . s c}$. becomes $m_{e} c^{2} / 2 \approx 250 \mathrm{keV}$. Finally, the interpolation from zero energy up to the backscattering energy is performed one channel at a time using the same channels in $C_{1}, C$, and $C_{2}$, independent of the angle.

\subsubsection{The Folding Iteration Method}

Once the response matrix is obtained for all of the $\gamma$-ray energies, the continuum $\gamma$-ray spectra of the respective excitation-energy windows are unfolded using the folding iteration method $[63,64]$. In the description of the method that follows, we will consider only one $\gamma$-ray spectrum. The observed or folded spectrum $(f)$ can be written as a product of the unfolded spectrum $(u)$ and the response matrix $(\mathbf{R})$

$$
f=\mathbf{R} u
$$


or in a matrix form

$$
\left(\begin{array}{c}
f_{1} \\
f_{2} \\
\vdots \\
f_{n}
\end{array}\right)=\left(\begin{array}{cccc}
R_{11} & R_{12} & \ldots & R_{1 n} \\
R_{21} & R_{22} & \ldots & R_{2 n} \\
\vdots & \vdots & \ldots & \vdots \\
R_{n 1} & R_{n 2} & \ldots & R_{n n}
\end{array}\right)\left(\begin{array}{c}
u_{1} \\
u_{2} \\
\vdots \\
u_{n}
\end{array}\right)
$$

The index from 1 to $\mathrm{n}$ denotes the channel number in the $\gamma$-ray spectrum. $R_{i j}$ is defined as the detector response in channel $i$ when the detector is hit by $\gamma$ rays with an energy corresponding to channel $j$. Since the contribution to a specific channel from the fullenergy events that are registered in smaller channels is zero, all of the elements below the diagonal of the matrix $\mathbf{R}$ are zero.

The response matrix and the folded spectrum are already known, and the unfolded spectrum remains to be found. The folding iteration method [64] starts with a trial unfolded spectrum, and in each iteration, it improves the unfolded spectrum until the observed (folded) spectrum is obtained. The method is performed in the following steps:

1. As a first trial to determine the unfolded spectrum, the observed spectrum $(r)$ is chosen, $u^{0}=r$.

2. The first folded spectrum is calculated using Eq. 5.17, $f^{0}=\mathbf{R} u^{0}$.

3. The next trial function is obtained by adding the difference spectrum $r-f^{0}$ to the original trial unfolded spectrum, $u^{1}=u^{0}+\left(r-f^{0}\right)$.

4. A new folded spectrum is calculated from $f^{1}=\mathbf{R} u^{1}$; again this is used in the next trial function, $u^{2}=u^{1}+\left(r-f^{1}\right)$, or in general, $u^{i+1}=u^{i}+\left(r-f^{i}\right)$ 
where $i$ is the iteration index. The iteration continues until $f^{i} \approx r$. In this procedure, using a response matrix with the experimental energy resolution gives artificial undershoots on both sides of pronounced peaks in the unfolded spectrum [63, 64]. Therefore, as suggested by Ref. [64], the full-width at half maximum for the response matrix is taken as half of the experimental FWHM, i.e. $\mathrm{FWHM}^{\text {resp }}=0.5 \mathrm{FWHM}^{\text {exp }}$, for the folding iteration method.

The general shape of the unfolded spectrum obtained this way is reliable, but the counts on both side of the prominent peaks fluctuate strongly from channel to channel. This is not surprising because finding the numerical inversion of the response matrix in $u=\mathbf{R}^{-1} f$ is highly unstable. A small variation in $\mathbf{R}$ results in a large variation of $\mathbf{R}^{-\mathbf{1}}$. Therefore, we will use the Compton subtraction method [63] to prevent the oscillations in the unfolded spectrum obtained with the folding iteration method.

\subsubsection{The Compton Subtraction Method}

The Compton subtraction method [63] smoothes and subtracts the Compton background from the observed spectrum. In this procedure, the unfolded spectrum of the folding iteration method is taken as a starting point, and denoted with $u_{0}$.

First, the full-energy, single-escape, double-escape, and annihilation events are folded using the detector response; i.e.,

$$
\begin{aligned}
f_{f}(i) & =p_{f} u_{0}(i) \\
f_{s}(i) & =p_{s}\left(i+i_{511}\right) u_{0}\left(i+i_{511}\right) \\
f_{d}(i) & =p_{d}\left(i+i_{1022}\right) u_{0}\left(i+i_{1022}\right) \\
f_{a}\left(i_{511}\right) & =\sum_{i} p_{a}(i) u_{0}(i) .
\end{aligned}
$$

The $p_{f}, p_{s}, p_{d}$, and $p_{a}$ are taken from Table 5.1. The index $i$ represents the channel for the full-energy peak, and $i_{511}$ and $i_{1022}$ are the channels having energies 511 and $1022 \mathrm{keV}$. The annihilation events are all registered in channel $i_{511}$ since the annihilation $511-\mathrm{keV} \gamma$ rays originate in the detector surroundings. 
Next, each of these folded contributions is smoothed with an appropriate energy resolution. Note that the energy resolution of the unfolded spectrum in the folding iteration method was changed with $\mathrm{FWHM}^{\text {resp }}=0.5 \mathrm{FWHM}^{\exp }$, and thus the spectrum $u_{0}$ has FWHM $=\sqrt{1.0^{2}-0.5^{2}}=0.87 \mathrm{FWHM}^{\text {exp }}$. Therefore, the $f_{f}, f_{s}$, and $f_{d}$ spectra are smoothed with an additional 0.5 FWHM Fxp $^{e x}$ order to obtain the experimental energy resolution $\left(\sqrt{0.87^{2}+0.5^{2}} \approx 1\right)$. The $f_{a}$ is smoothed with a resolution of $1.0 \mathrm{FWHM}^{\text {exp }}$. The spectra smoothing is performed with a Gaussian function by going $6 \sigma$ on each side of the centroid. Thus, channels close to the centroid are weighted higher than channels away from the centroid.

The new $f_{f}, f_{s}, f_{d}$, and $f_{a}$ spectra are then subtracted from the observed spectrum $r$, and the Compton background is obtained

$$
f_{c}(i)=r(i)-\left[f_{f}(i)+f_{s}(i)+f_{d}(i)+f_{a}\left(i_{511}\right)\right]
$$

Note that the term $f_{a}\left(i_{511}\right)$ will be zero unless $i=i_{511}$. The Compton background makes a relatively large contribution to the detector response. Therefore, the extraction of $f_{c}(i)$ with Eq. 5.19 reduces the fluctuations caused by the folding iteration method. The spectrum $f_{c}$ still shows strong oscillations due to statistical fluctuations in the observed spectrum. Thus the spectrum $f_{c}(i)$ can be smoothed with a large energy resolution, e.g 1.0 FWHM $^{\text {exp }}$ because the Compton background is a slowly-varying function of incident $\gamma$-ray energy.

Next, the new unfolded spectrum is obtained by subtracting $f_{c}, f_{s}, f_{d}, f_{a}$ from the observed spectrum $r$, and dividing it by the full-energy probability

$$
u_{f}(i)=\frac{1}{p_{f}(i)}\left[r(i)-f_{c}(i)-f_{s}(i)-f_{d}(i)-f_{a}\left(i_{511}\right)\right]
$$

The new unfolded spectrum has the same statistical fluctuations as the observed spectrum as a result of smoothing the Compton part. As a final step the new unfolded spectrum is 
corrected with the total $\gamma$-ray detection efficiency

$$
U_{f}(i)=\frac{u_{f}(i)}{\epsilon_{t o t}(i)}
$$

where $\epsilon_{t o t}$ is the total $\gamma$-ray efficiency taken from Table 5.1 multiplied with the efficiency due to the discriminator level of the experimental setup. This efficiency depends on, e.g., timing, ADC thresholds, and the absorber material used for the $\gamma$-ray detectors.

An unfolded $\mathrm{E}_{x}$ vs. $\mathrm{E}_{\gamma}$ matrix is obtained for the ${ }^{56} \mathrm{Fe}$ and ${ }^{57} \mathrm{Fe}$ isotopes using the folding iteration method followed by the Compton subtraction method. One weakness of the unfolding method appears when the full-energy peak obtained in Eq. 5.20 is divided by $p_{f}\left(p_{f}<1\right)$. The full-energy peak is a Gaussian peak, and by dividing this peak by $p_{f}$, we divide every point on the Gaussian with the same $p_{f}$. Since the error of each channel is also divided by the same number this causes fluctuations in the data; this problem cannot be easily solved.

\subsection{Extracting Primary $\gamma$-ray Spectra}

Population of each level in the residual nucleus results in a $\gamma$-ray cascade, and we are interested only in the primary or the first $\gamma$ ray in this cascade. Because the timing between the $\gamma$ rays in a cascade cannot be resolved experimentally, these $\gamma$ rays are observed simultaneously; as a result one cannot know which $\gamma$ ray came first in that cascade. The separation of the primary and the rest of the cascade can be achieved by a subtraction method [65]. This method will be applied to the unfolded $E_{x}-E_{\gamma}$ matrix.

The basic idea of extracting the primary $\gamma$ rays is simply illustrated in Fig. 5.12. Assume that the ${ }^{57} \mathrm{Fe}\left({ }^{3} \mathrm{He}, \alpha\right)$ reaction populates the residual ${ }^{56} \mathrm{Fe}$ nucleus at the level $E_{i}$, and this level depopulates by a $\gamma_{1}-\gamma_{2}-\gamma_{3}$ cascade. The $\gamma$-ray spectrum $f_{i}$ gated on the level $E_{i}$ in the unfolded matrix will include all of the transitions in this cascade. Similarly, if the same reaction populates the level $E_{2}$ the same number of times $\left(\sigma_{i}=\right.$ $\sigma_{2}$ ), the corresponding gated spectrum $f_{2}$ includes the $\gamma_{2}-\gamma_{3}$ cascade. The primary $\gamma$-ray 


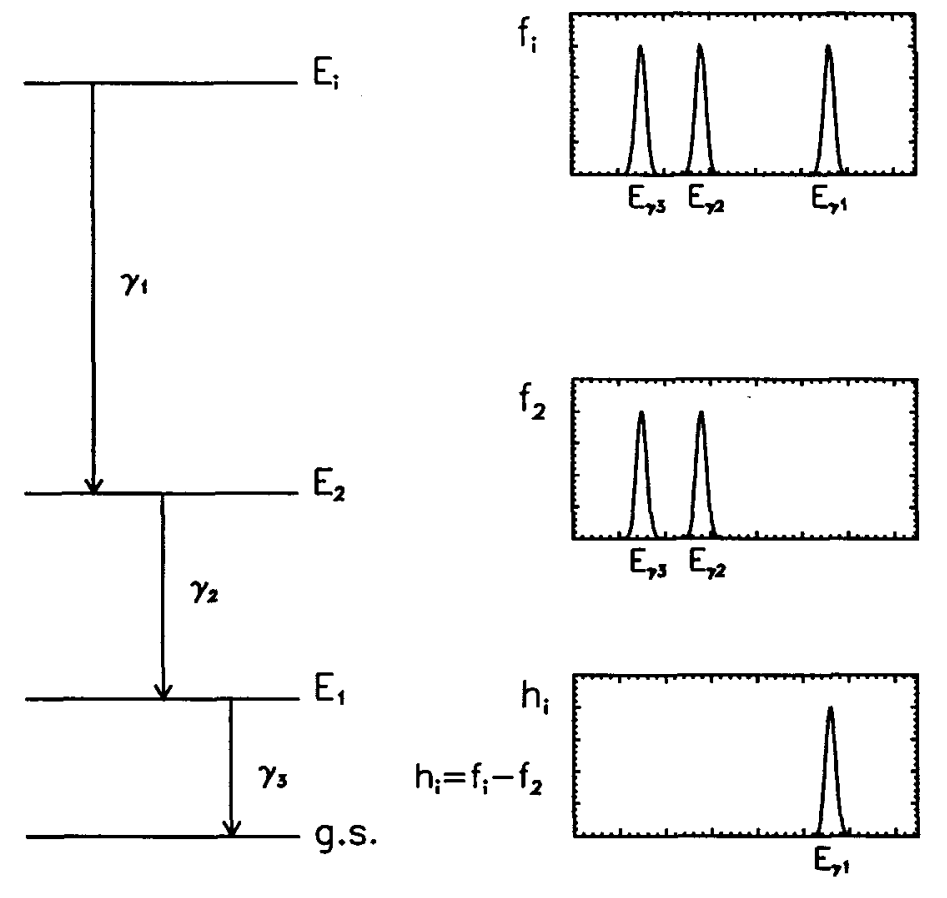

Figure 5.12: Schematic representation of the subtraction method.

spectrum $h_{i}$ for the level $E_{i}$ can be obtained by subtracting the spectrum $f_{2}$ from the spectrum $f_{i}$, as shown in the lower part of Fig. 5.12

$$
h_{i}=f_{i}-f_{2}
$$

Clearly, this spectrum includes only the primary $\gamma_{1}$ rays. In Fig. 5.12, we assumed that the cross sections for populating the levels $E_{i}$ and $E_{2}$ are equal. In reality, they are different. Therefore one must take into account the difference in these cross sections in the subtraction method. We can do this using the singles particle spectrum since the particles are measured in the singles mode as well as in the coincidence mode. The singles particle spectrum is proportional to the reaction yield (note that only those particles emitted at the $45^{\circ}$ forward direction are measured.) One can find the population of the 
level $E_{2}$ relative to the level $E_{i}$ by simply dividing the respective cross sections

$$
n=\frac{\sigma_{i}}{\sigma_{2}}
$$

The spectrum $f_{2}$ has to be multiplied by the normalization coefficient $n$ in order to compensate for the different cross sections

$$
h_{i}=f_{i}-n f_{2}
$$

For example, if the level $E_{2}$ is populated half as many times as is the level $E_{i}$, the spectrum $f_{2}$ is multiplied by two.

Thus far, we assumed only one $\gamma$ transition from the levels $E_{1}, E_{2}$, and $E_{i}$ for simplicity. Of course, an excited state can decay via several different branches, as shown in Fig. 5.13. In that case, one needs to generalize the above procedure for additional cascades. Assume that the level $E_{i}$ decays by $\gamma_{1}, \gamma_{2}, \gamma_{3}, \ldots, \gamma_{j}$ with decay probabilities $w_{1}^{i}, w_{2}^{i}, w_{3}^{i}, \ldots, w_{j}^{i}$, respectively. Then one must subtract the $\gamma$-ray spectra $f_{j}$ for the corresponding levels $\mathrm{E}_{j}$ from the spectrum $f_{i}$ to obtain the primary $\gamma$-ray spectrum for the level $E_{i}$. Before the subtraction, the spectra $f_{j}$ have to be weighted with $w_{j}^{i}$. This can be written as

$$
h_{i}=f_{i}-\left[n_{1}^{i} w_{1}^{i} f_{1}+n_{2}^{i} w_{2}^{i} f_{2}+n_{3}^{i} w_{3}^{i} f_{3}+\ldots+n_{j}^{i} w_{j}^{i} f_{j}\right]
$$

or in general

$$
h_{i}=f_{i}-g_{i},
$$




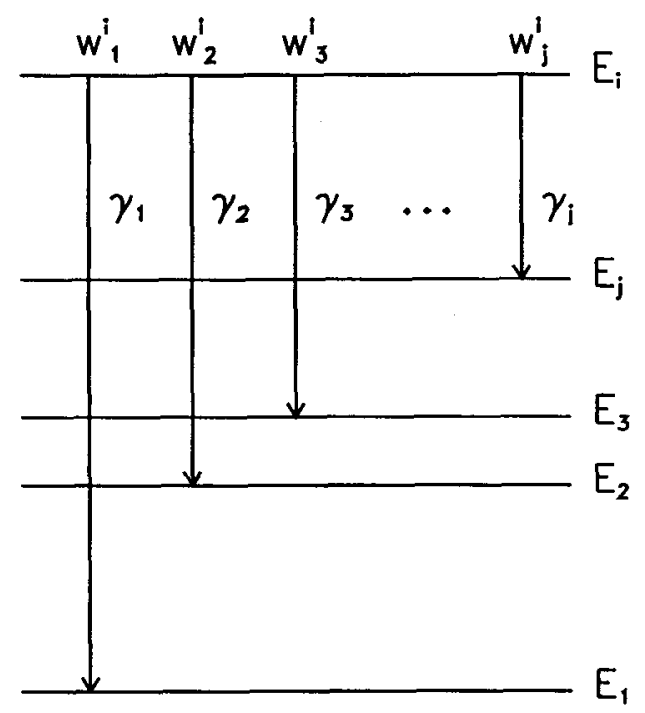

g.s.

Figure 5.13: $\gamma$ rays depopulating the level $\mathbf{E}_{i}$.

where

$$
g_{i}=\sum_{j} n_{j}^{i} w_{j}^{i} f_{j}, \text { and } n_{j}^{i}=\sigma_{i} / \sigma_{j}
$$

The coefficients $w_{j}^{i}$ are closely related to the primary $\gamma$-ray spectrum $h_{i}$. In fact, if the spectrum $h_{i}$ is normalized to one, the resulting spectrum gives the coefficients $w_{j}^{i}$, which will be called the weighting functions hereafter.

Figure 5.14 provides an insight toward how this normalization is performed. The decay scheme of the level $E_{i}$ is shown on the left side of Fig. 5.14. Here the population cross sections are assumed to be equal for simplicity $\left(\sigma_{i}=\sigma_{1}=\sigma_{2}\right)$. In this case, the $E_{i}$ gated spectrum $f_{i}$ is comprised of $\gamma_{1}, \gamma_{2}, \gamma_{3}, \gamma_{4}$. The corresponding peak areas are denoted by $A_{1}, A_{2}, A_{3}, A_{4}$. The sum of $A_{3}$ and $A_{4}$ gives the peak area for the primary $\gamma$ rays. The peak areas for those $\gamma$ rays that belong to the same cascade will be the same 

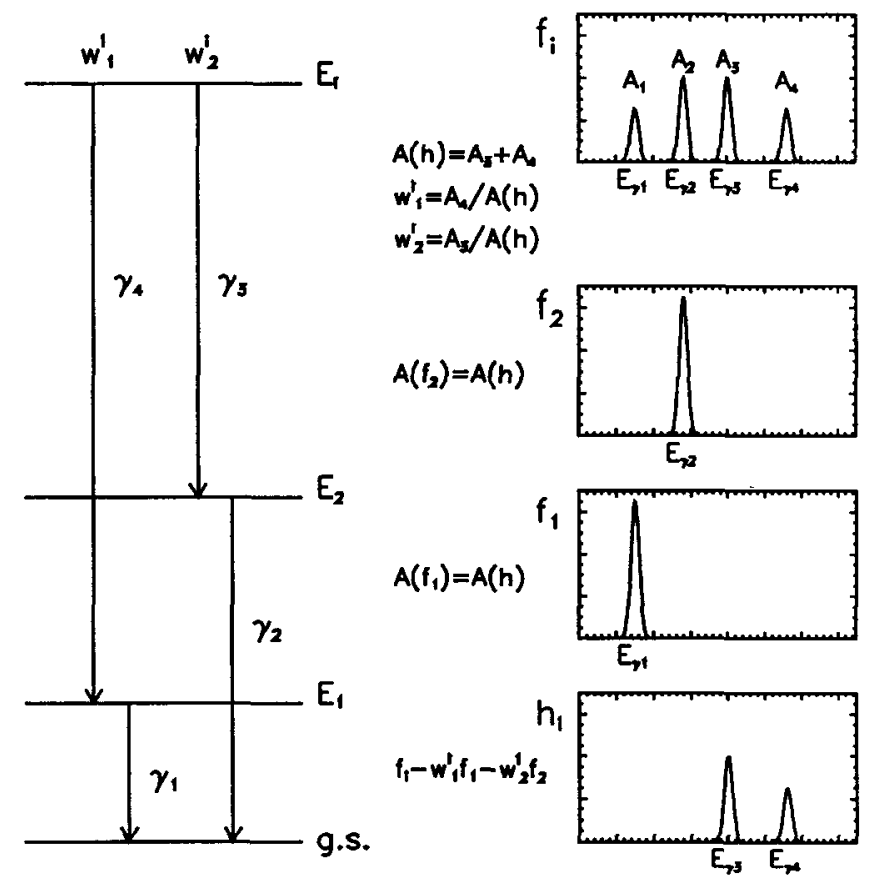

Figure 5.14: Determination of the weighting function.

in the spectrum $f_{i}$, i.e., $A_{1}=A_{4}$ and $A_{2}=A_{3}$. The probability coefficients $w_{1}^{i}$ and $w_{2}^{i}$ are then obtained by normalizing the peak area $A_{3}$ and $A_{4}$, respectively. Because $\sigma_{i}=\sigma_{1}$ $=\sigma_{2}$, the peak area of the spectrum $f_{1}$ and $f_{2}$ is equal to the peak area of the primary $\gamma$ rays $A(h)$. Therefore one multiplies the spectrum $f_{1}$ and $f_{2}$ by the corresponding $w_{1}^{i}$ and $w_{2}^{i}$ in the subtraction method. Then the resulting spectrum gives the primary $\gamma$ rays, as shown in Fig. 5.14. In all of these three examples we chose the $\gamma$ rays well separated for convenience. Of course, in reality the $\gamma$-ray spectrum and the weighting function have a continuum structure due to the large number of levels at higher excitation energy. Therefore the subtraction is performed channel by channel.

Because the $h_{i}$ and the $w_{j}^{i}$ are closely related, one could start with a trial weighting function and calculate the $h_{i}$ until the trial weighting function converges. In fact, this iteration process is proven to converge very fast in Ref. [65]. Furthermore, this method has been tested using different trial weighting functions [65]. It is shown that the shape of the primary $\gamma$-ray spectrum remains almost the same for different weighting functions 
although the area is subject to change [65]. In order to account for an improper choice of weighting function, an area correction is applied to Eq. 5.26

$$
h_{i}=f_{i}-\alpha g_{i}
$$

If the area of the spectrum $h_{i}$ equals the area of the spectrum $f_{i}$ minus the area of the spectrum $g_{i}$, then the coefficient $\alpha$ would be one. The determination of $\alpha$ can be found in Appendix C.

The algorithm followed in the code is the following:

1. start with a trial weighting function, for example, the detector response,

2. calculate $h_{i}$ from Eq. 5.28 ,

3. transform $h_{i}$ to $w_{j}^{i}$,

4. if $w_{j}^{i} \approx w_{j}^{i}(o l d)$, then the $h_{i}$ calculated from Eq. 5.28 with the new $w_{j}^{i}$ give the primary $\gamma$-ray spectrum for the level $E_{i}$. Otherwise, proceed with step 2.

Step 3 needs some explanation. First, note that the unfolded matrix is a twodimensional matrix. The calibration on each dimension $\left(E_{\gamma}\right.$ and $\left.E_{x}\right)$ is different because the experimental gain and shift for the $\mathrm{NaI}(\mathrm{Tl})$ and Si detectors are different. The spectra $h_{i}$ have the $E_{\gamma}$ calibration. However, in the calculation of $g_{i}$ in Eq. 5.27 we consider the $\gamma$-ray energy as being the energy difference between the initial and the final levels, meaning that the $E_{x}$ calibration is used. Therefore, one needs to change the calibration of the spectra $h_{i}$ from the $E_{\gamma}$ calibration to the $E_{x}$ calibration in Step 3. This is done with a subroutine in the code. Finally, the calibrated spectra $h_{i}$ are normalized to one, as explained with Fig. 5.14, in order to obtain the next trial spectra $w_{j}^{i}$.

The subtraction method relies on a number of assumptions. The main assumption is that the $\gamma$-ray decay pattern from any excitation-energy bin is independent of the population mechanisms, i.e., populated directly by the $\left({ }^{3} \mathrm{He}, \alpha\right)$ or $\left({ }^{3} \mathrm{He}^{3} \mathrm{He}^{\prime}\right)$ reactions or 
populated indirectly by a $\gamma$-ray decay that originates in higher excited states following the initial nuclear reaction. This is presumably fulfilled if

1. full thermalization is achieved,

2. the reaction populates approximately the same spins at each excitation interval,

3. there are about equal numbers of positive and negative parity states populated in each interval.

The $\left({ }^{3} \mathrm{He}, \alpha\right)$ reaction is a direct neutron pick-up reaction. The reaction takes place through the single neutron components of the wave function of the target nucleus. The formation of a complete eigenstate is assumed to be a very fast process $\left(\approx 10^{-18}\right)$ compared to the time necessary for photon emission $\left(\approx 10^{-15}\right)$. Then the excited nucleus forgets how it was formed, and the decay process is statistical. Thus the full thermalization is expected to occur. However, this is not necessary as long as the decay pattern of states remains unchanged independent of the way the states were formed. Due to strong singleparticle effects at low excitations, the photon emission is not statistical, but affected by the discrete structure. Therefore, the low-lying levels will not be used in obtaining the level density and the $\gamma$-ray strength function.

The second and third assumptions are investigated in Ref. [66] in detail. Here only the conclusions drawn in Ref. [66] will be mentioned. In order to obtain information about the spin distribution for each excitation energy interval, the intensity of the groundstate band feeding is investigated as a function of excitation energy [66]. This method is described in Refs. $[67,68]$. In each step in the cascade the spin distribution becomes wider, but the average spin remains approximately constant. With an average statistical multiplicity of 3 or less, the side feeding to the ground-state band is expected to reflect the initial spin distribution. It was shown that the intensity of the side feeding to the ground-state band transitions remains approximately the same over all excitation energies [66]. Therefore it is concluded that the spin population is approximately the same over the energy region under consideration [66]. 
The dominance of the E1 dipole transitions from the states populated in the continuum results in successive parity changes in the cascade. As a result, $\gamma$ transitions subtracted from the $\gamma$-ray spectra in the subtraction method originate in states with opposite parity. There is no problem if the population of both parities is approximately equal. In fact, this was found to be the case for low excitation energies [69]. One may expect this to be approximately true over the entire energy range due to damping of the single-particle strength. In addition, even a small portion of M1 transitions would reduce this possible problem [66]. However, one cannot completely justify the arguments of Ref. [66]. Spin and parity effects should be investigated further, and may result in corrections to the subtraction method.

The raw, unfolded, and primary $\gamma$-ray spectra are displayed in Fig. 5.15 for ${ }^{56} \mathrm{Fe}$ (top panels) at 5.0-MeV excitation energy and ${ }^{57} \mathrm{Fe}$ (bottom panels) at 6.2-MeV excitation energy.

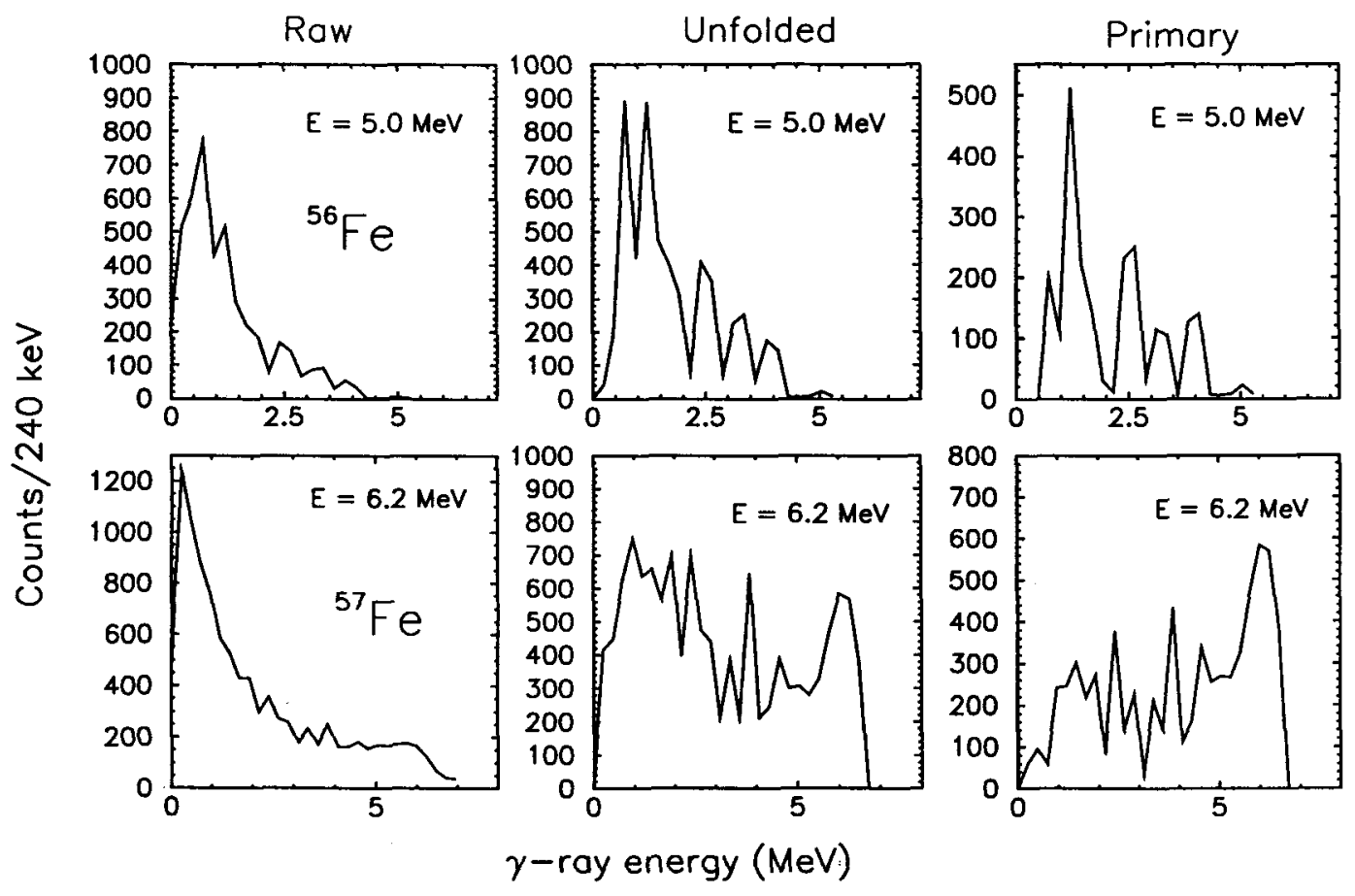

Figure 5.15: Raw (left), unfolded (middle), and primary (right) $\gamma$-ray spectra at $E_{x}=5.0 \mathrm{MeV}$ for ${ }^{56} \mathrm{Fe}$ (top) and at $E_{x}=6.2 \mathrm{MeV}$ for ${ }^{57} \mathrm{Fe}$ (bottom). 
$\prod_{\text {CHAPTER }}$

\section{Experimental Results on ${ }^{56} \mathrm{Fe}$ and ${ }^{57} \mathrm{Fe}$}

\subsection{Level Densities}

The level density and radiative strength function for ${ }^{56} \mathrm{Fe}$ and ${ }^{57} \mathrm{Fe}$ are extracted from the experimental primary $\gamma$-ray spectra assuming the Axel-Brink hypothesis. This method has already been described in Chapter 3. Recall that both the level density and $\gamma$-ray strength function obtained from the $\chi^{2}$ minimization is only one solution among an infinite number of solutions that reproduces the primary $\gamma$-ray matrix. The most probable solution is found by normalization to known data [70]. The details of this procedure are given here.

It has been proven in Appendix A that all solutions are related to each other by the transformation

$$
\begin{aligned}
\tilde{\rho}\left(E_{i}-E_{\gamma}\right) & =\rho\left(E_{i}-E_{\gamma}\right) A \exp \left(\alpha\left(E_{i}-E_{\gamma}\right)\right) \\
\tilde{T}\left(E_{\gamma}\right) & =T\left(E_{\gamma}\right) B \exp \left(\alpha E_{\gamma}\right)
\end{aligned}
$$

where $A, B$, and $\alpha$ are free parameters, $T$ and $\rho$ are one of the solutions obtained from the least $\chi^{2}$ method, and $\tilde{T}$ and $\tilde{\rho}$ are the other solutions. In order to find the most physical level density and $\gamma$-ray transmission coefficient, say $\tilde{\rho}$ and $\tilde{T}$, one needs to determine appropriate parameters $A, B$, and $\alpha$.

We use two anchor points for normalization of the level density: the known dis- 
crete levels at low excitation energy and the level density at the neutron binding energy determined from the neutron-resonance data. The parameter $\alpha$ determines the slope of the level density $\rho$ and the $\gamma$-ray transmission coefficient $T$. The parameters $A$ and $B$ determine the magnitude of $\rho$ and $T$, respectively. Thus one needs to determine both the slope (the parameter $\alpha$ ), and the magnitude (the parameter $A$ ) for the level density and the parameter $B$ for the $\gamma$-ray strength function.

In the top panel of Fig. 6.1, the known discrete levels per excitation-energy bin $(238 \mathrm{keV})$ are shown as a histogram. The excitation-energy region between the arrows are used for the normalization. States below $2 \mathrm{MeV}$ are not included, since statistical assumptions break down in the region close to the nuclear ground state. The level density at the neutron binding energy is shown as an open triangle in the lower panel of Fig. 6.1. Unfortunately the experimental level density cannot be directly compared to the level density at the neutron binding energy $\left(B_{n}\right)$, since the level density is measured up to $E_{x}=B_{n}-1.5 \mathrm{MeV}$. Therefore, we extrapolate the experimental level density to the neutron binding energy using the back-shifted Fermi gas model

$$
\rho(E)=\frac{e^{2 \sqrt{a\left(E-E_{1}\right)}}}{12 \sqrt{2} \sigma a^{1 / 4}\left(E-E_{1}\right)^{5 / 4}}
$$

where the back-shift parameter is given by $E_{1}=C_{1}+\Delta$, and the parameters $a$ and $C_{1}$ are calculated using von Egidy's parameterization [71]

$$
\begin{aligned}
a & =0.21 A^{0.87} \mathrm{MeV}^{-1} \\
C_{1} & =-6.6 A^{-0.32} \mathrm{MeV} .
\end{aligned}
$$

Furthermore, the parameter $\Delta$ is calculated from the mass formula [72], and the spin-cutoff parameter of Gilbert and Cameron [6] is employed 

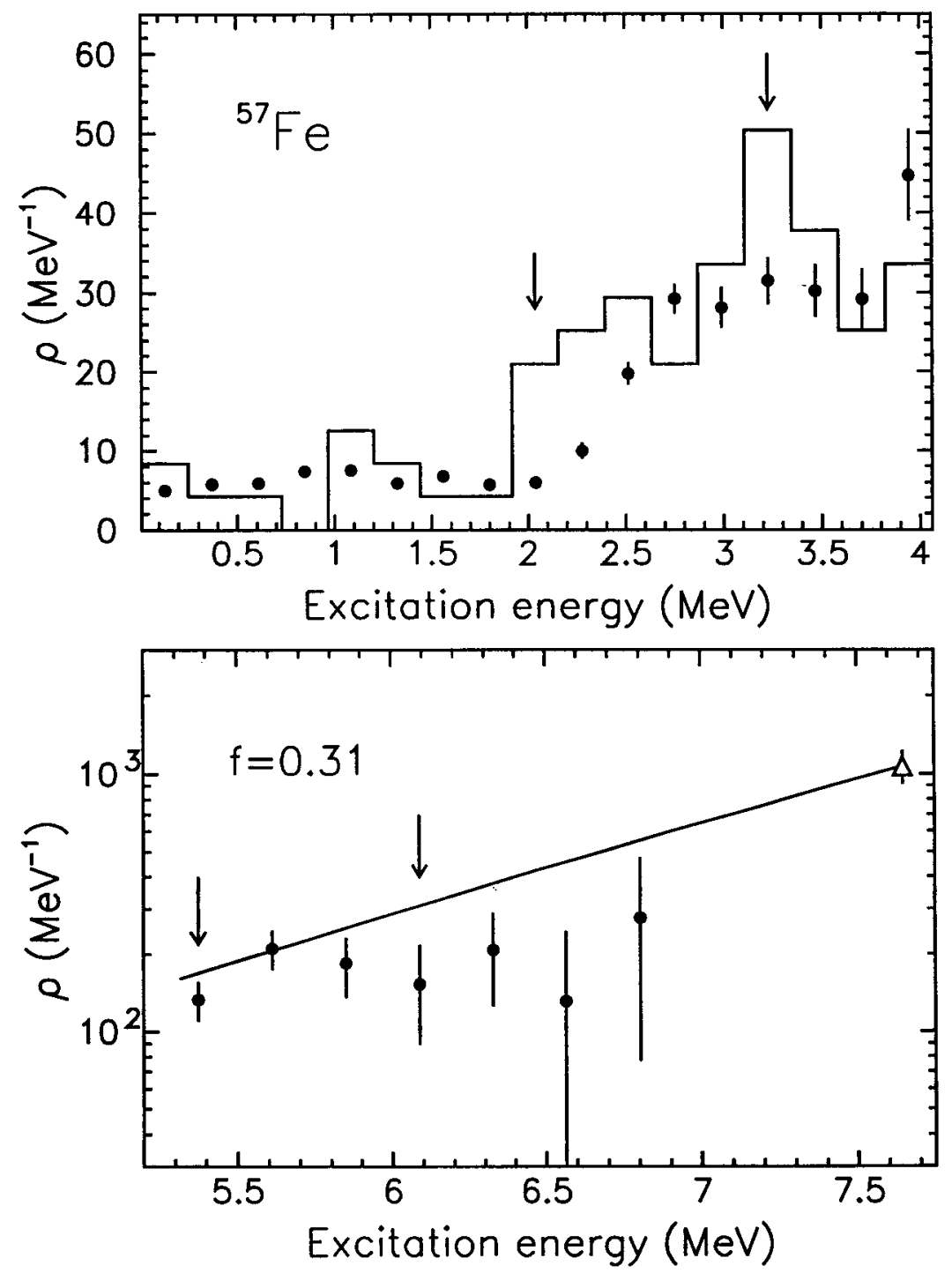

Figure 6.1: Normalization of the ${ }^{57}$ Fe level density. The known discrete levels are shown as a histogram in the top panel. The open triangle in the lower panel gives the level density at the neutron binding energy $B_{n}$. The normalized experimental level density is shown by solid circles. The factor $f$ is obtained by a comparison between the level density calculated from the Fermi gas model and the experimental level density at $B_{n}$. The solid line is obtained by extrapolating the experimental level density to $B_{n}$ using the Fermi gas model. 


$$
\sigma^{2}=0.0888 A^{2 / 3} \sqrt{a\left(E-E_{1}\right)}
$$

In order to extrapolate the experimental level density, first the level density calculated from the Fermi gas model is compared with the level density at the neutron binding energy. Then the Fermi-gas level density is multiplied by the factor (0.31) obtained from this comparison, thus, is forced to match the neutron resonance data. The extrapolated level density in the excitation-energy region between $5.4 \mathrm{MeV}$ and $6.1 \mathrm{MeV}$ is used for the normalization procedure. The data points in both panels in Fig. 6.1 are the normalized experimental level densities. The Fermi-gas extrapolation of the level density is shown by a solid line.

Similarly, known discrete levels in ${ }^{56} \mathrm{Fe}$ are shown as a histogram in Fig. 6.2. Since ${ }^{55} \mathrm{Fe}$ is an unstable nucleus $\left(T_{1 / 2}=2.73 \mathrm{y}\right)$, there is no available neutron-resonance data for the ${ }^{56} \mathrm{Fe}$ isotope. Therefore, the level density in ${ }^{56} \mathrm{Fe}$ is normalized using information from the neighboring ${ }^{57} \mathrm{Fe}$ isotope. The normalization is performed by applying the factor $\mathrm{f}$, obtained by comparing the level density at $B_{n}$ in ${ }^{57} \mathrm{Fe}$ with the one calculated from the Fermi gas model using von Egidy's parameterization, to the von Egidy Fermi-gas level density of ${ }^{56} \mathrm{Fe}$. The data points between the arrows in Fig. 6.2 are used for the normalization.

The normalization parameters $A$ and $\alpha$ for the level density are determined by fitting the ratio $\tilde{\rho} / \rho$ with the transformation function $A \exp \left(\alpha\left(E_{x}-E_{\gamma}\right)\right)$. Once $A$ and $\alpha$ are found from the fit the normalized level density is simply obtained by multiplying $\rho$ by the transformation function $A \exp \left(\alpha\left(E_{x}-E_{\gamma}\right)\right)$.

The normalized level densities for ${ }^{56} \mathrm{Fe}$ (solid circles) and ${ }^{57} \mathrm{Fe}$ (open circles) are shown in Fig. 6.3. The open triangle at $E_{x}=7.646 \mathrm{MeV}$ is the level density at $B_{n}$ for ${ }^{57} \mathrm{Fe}$. The normalized Fermi-gas level density, shown by solid lines, describes the shape of the experimental data quite well in this energy region. The fact that the level density in ${ }^{57} \mathrm{Fe}$ is higher than the one in ${ }^{56} \mathrm{Fe}$ is the result of the unpaired neutron in ${ }^{57} \mathrm{Fe}$. A 

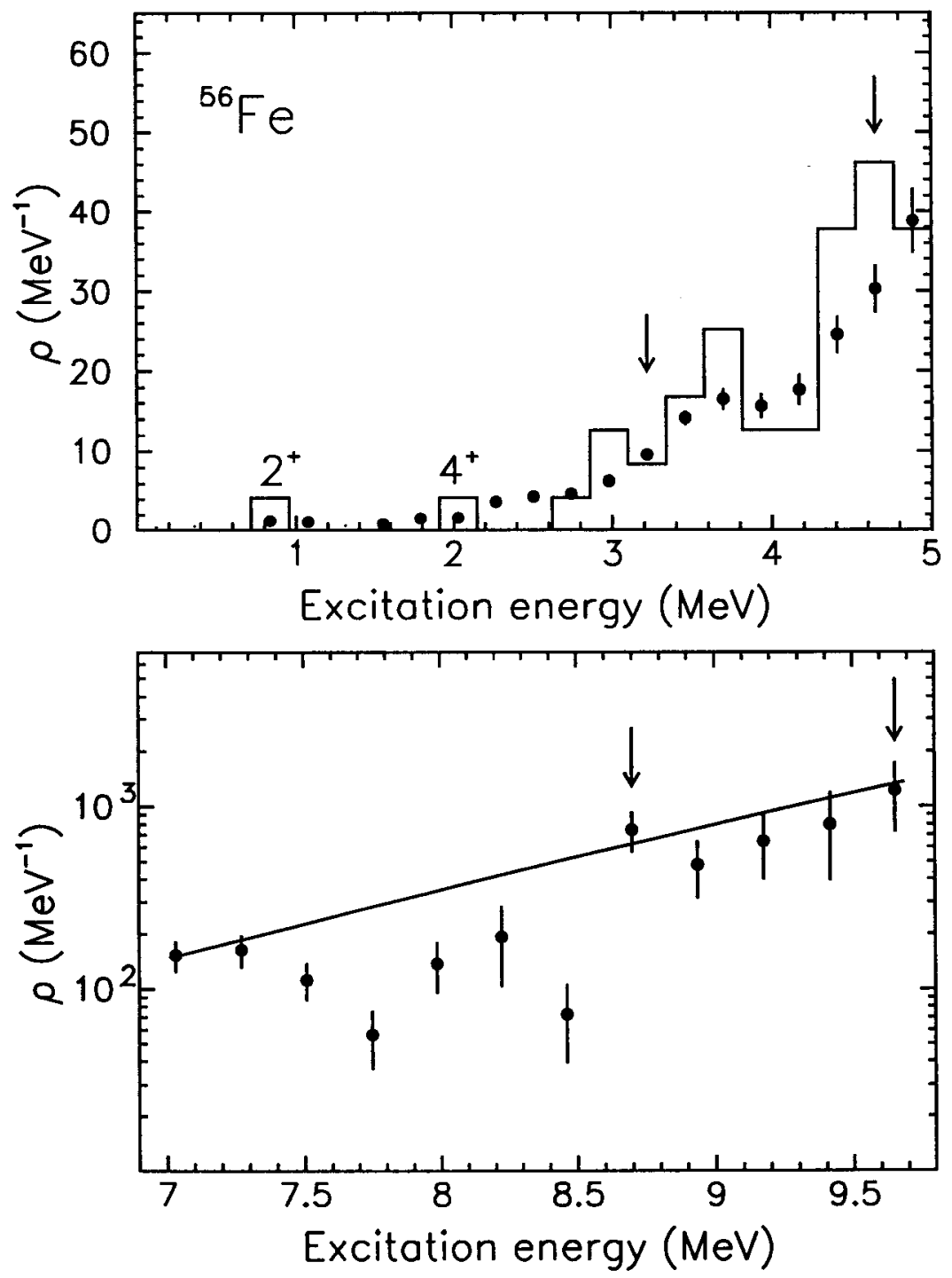

Figure 6.2: Same as Fig. 6.1 in ${ }^{56} \mathrm{Fe}$. The factor $f=0.31$ in the ${ }^{57} \mathrm{Fe}$ case is employed in the normalization of the ${ }^{56} \mathrm{Fe}$ level density. See text for details. 
common feature for both ${ }^{56} \mathrm{Fe}$ and ${ }^{57} \mathrm{Fe}$ in Fig. 6.3 is the step structure in the level density at low $E_{x}$. Discrete-level effects are also pronounced in ${ }^{56} \mathrm{Fe}$ at low excitation energy. For example, the first bump at $847 \mathrm{keV}$ corresponds to the first excited state (see Fig. 6.2 ), and the second excited $4^{+}$state appears at around $2 \mathrm{MeV}$. Between 2- and 3-MeV excitation energies, a rather flat plateau-like behavior is observed, then a step follows this plateau at around $3 \mathrm{MeV}$. This step structure is a signature for the first pair breaking in ${ }^{56} \mathrm{Fe}$, and can be associated with the predicted pairing-gap parameter $\Delta$. The parameter $\Delta$ is calculated using two different approaches, one is taken from Bohr and Mottelson [72], which uses a four-mass indicator, and the other is taken from Dobaczewski et al. [73], which uses a three-mass indicator. Simple arguments made in Ref. [73] show that one can avoid mixing the contributions from single-particle structure and pairing correlations by using the three-mass indicator for odd- $N$ and odd- $Z$ nuclei. Higher-mass indicators mix the pairing and single-particle contributions to odd-even mass staggering. Since only the pairing contribution is of interest, the values calculated from Ref. [73] are adopted in Fig. 6.3. The calculated pairing gap parameters are listed in Table 6.1 for both isotopes.

Table 6.1: The pairing gap parameters calculated from the Dobaczewski et al. [73] and Bohr and Mottelson approaches [72].

\begin{tabular}{c|cc}
\hline \hline & $\Delta_{p}(\mathrm{MeV})-{ }^{57} \mathrm{Fe}$ & $\Delta_{p}+\Delta_{n}(\mathrm{MeV})-{ }^{56} \mathrm{Fe}$ \\
\hline Dobaczewski et al. & 0.82 & 2.27 \\
Bohr and Mottelson & 1.27 & 2.93 \\
\hline
\end{tabular}

The first pair breaking is expected at $\Delta_{p}+\Delta_{n}$ in ${ }^{56} \mathrm{Fe}$. The calculated $\Delta_{p}+\Delta_{n}$ is approximately $0.5 \mathrm{MeV}$ below the energy of the first step at around $3 \mathrm{MeV}$. This is within reasonable agreement with the interpretation of the first pair breaking because, in addition to the pairing energy, an energy on the order of the single-particle energy is required in order to break one pair and excite one of the nucleons into the lowest unoccupied singleparticle state. The step structures associated with further pair breaking become less and less distinct with increasing excitation energy.

The discrete structure at low excitation in ${ }^{57} \mathrm{Fe}$ is not as pronounced as in ${ }^{56} \mathrm{Fe}$. Again this is because of the unpaired neutron in ${ }^{57} \mathrm{Fe}$. It is commonly believed that 


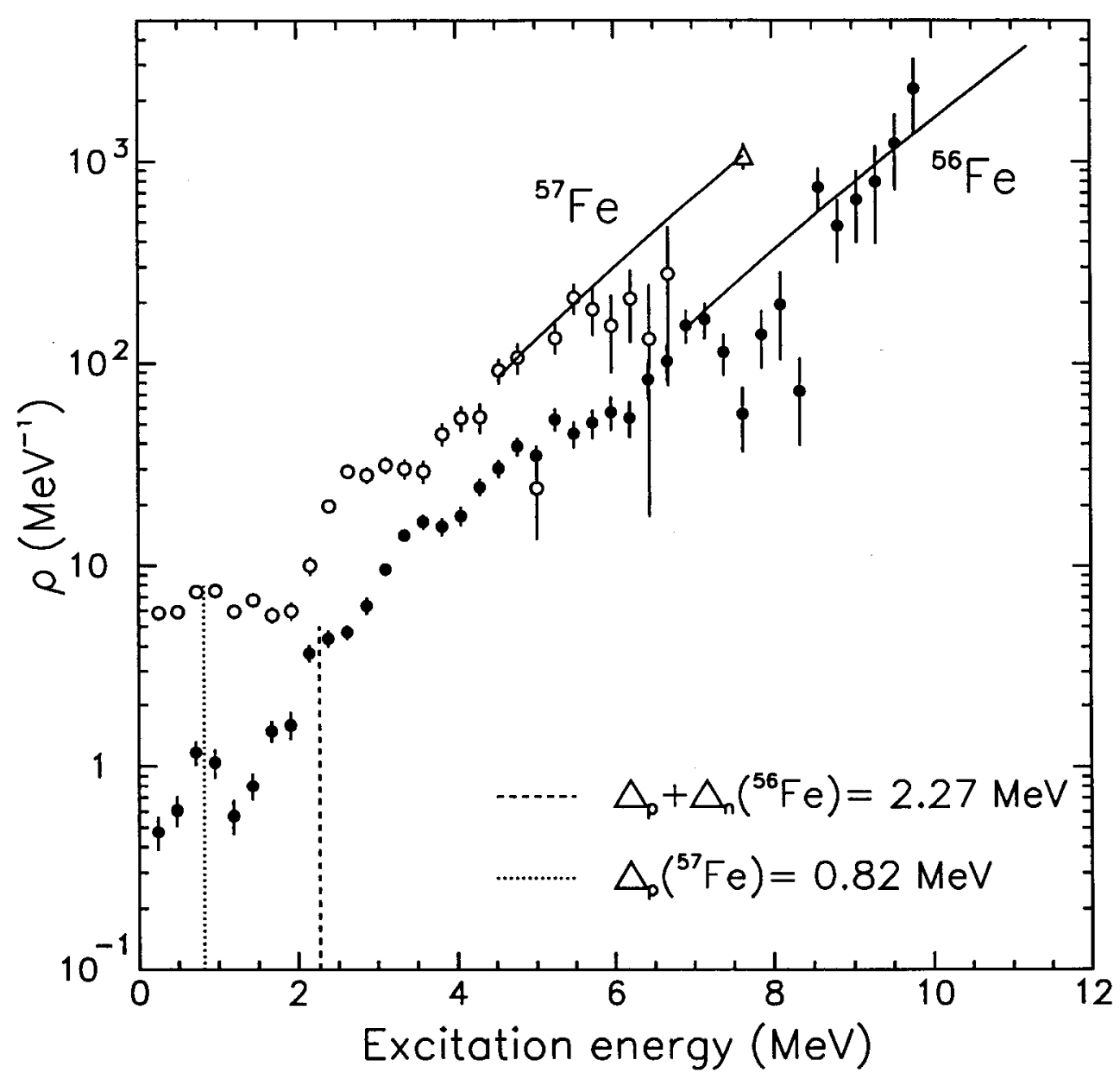

Figure 6.3: Level densities for ${ }^{56} \mathrm{Fe}$ and ${ }^{57} \mathrm{Fe}$.

neighboring odd-odd, odd-even, and even-even isotopes reveal the same level density if a proper shift is applied to the excitation energy [74]. The step structure at around $2 \mathrm{MeV}$ in ${ }^{57} \mathrm{Fe}$ would then correspond to the step observed at around $3 \mathrm{MeV}$ in ${ }^{56} \mathrm{Fe}$, taking into account an energy shift of about $1 \mathrm{MeV}$. Accordingly, the step at $2 \mathrm{MeV}$ is interpreted as the first pair breaking in ${ }^{57} \mathrm{Fe}$.

\subsection{Radiative Strength Functions}

Once the parameter $\alpha$ in Eq. 6.1 is obtained from the level density normalization, the slope of the $\gamma$-ray transmission coefficient is fixed by multiplying $T$ in Eq. 6.1 by the function 


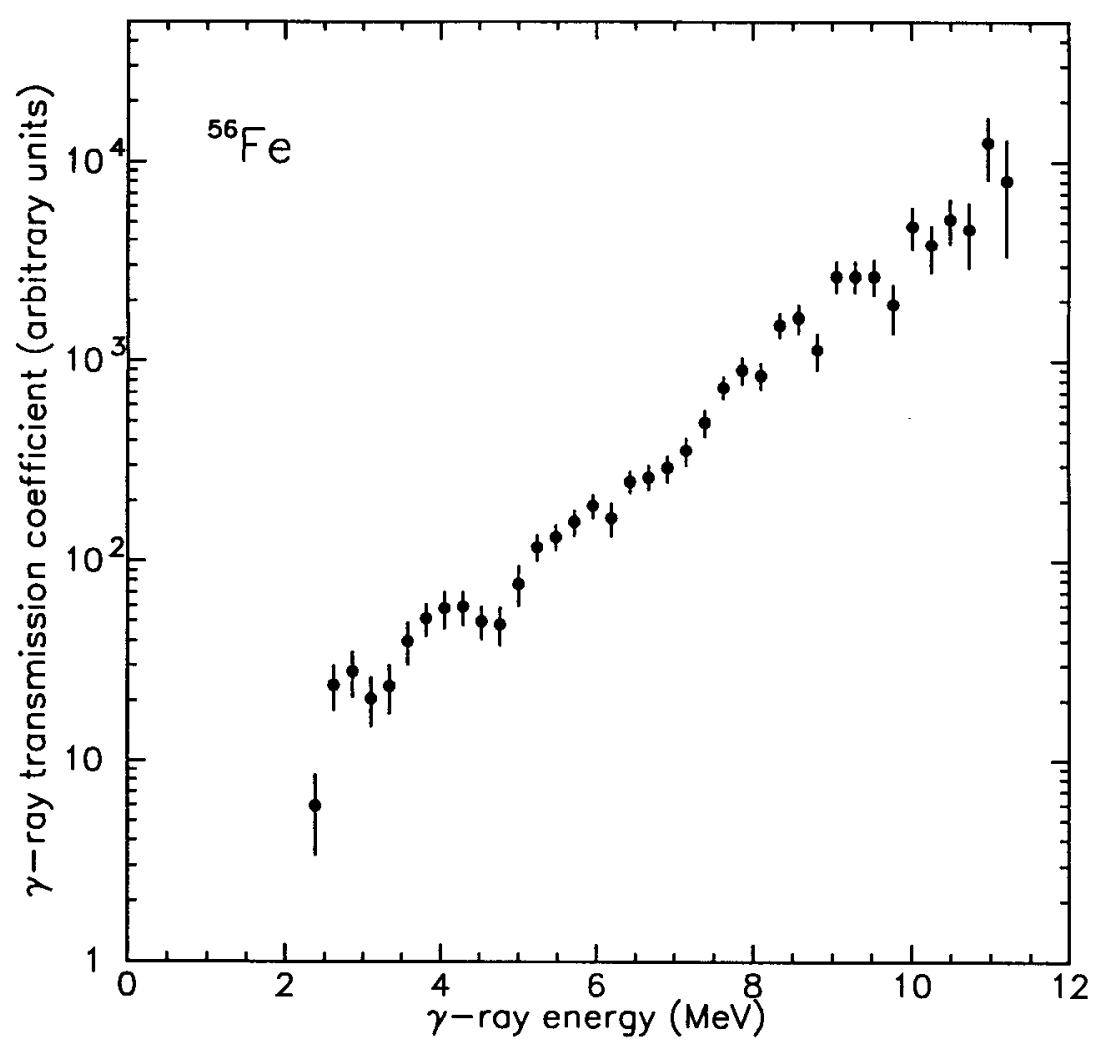

Figure 6.4: $\gamma$-ray transmission coefficient for ${ }^{56} \mathrm{Fe}$.

$\exp \left(\alpha E_{\gamma}\right)$. In Figs. 6.4 and 6.5, the $\gamma$-ray transmission coefficients with corrected slopes are shown for ${ }^{56} \mathrm{Fe}$ and ${ }^{57} \mathrm{Fe}$ isotopes, respectively. The magnitude, i.e., the normalization constant $B$ in Eq. 6.1, is determined using the average total radiative width of neutron resonances. Unfortunately, there is no experimental neutron-capture data for ${ }^{56} \mathrm{Fe}$, and thus no average total radiative width of neutron resonances. Therefore, the parameter $B$ cannot be determined for ${ }^{56} \mathrm{Fe}$. Here we will describe the normalization procedure for the ${ }^{57} \mathrm{Fe}$ isotope.

The $\gamma$-ray transmission coefficients $T_{X L}\left(E_{\gamma}\right)$ for multipole type $X L$ are related to the corresponding strength function by 


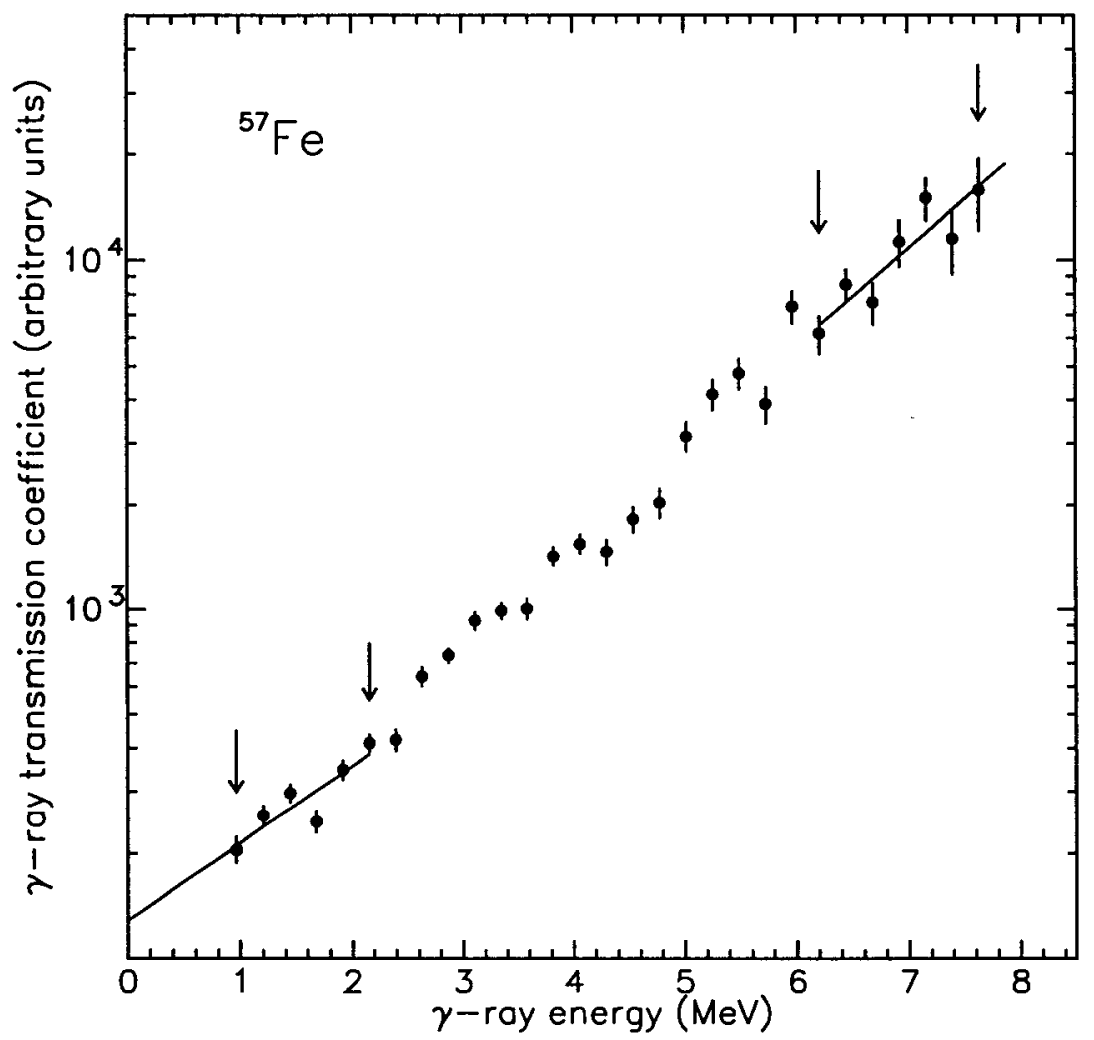

Figure 6.5: $\gamma$-ray transmission coefficient for ${ }^{57} \mathrm{Fe}$. The solid line is the extrapolation of the $\gamma$-ray transmission coefficient $T$ using the data points between the arrows. See text for details.

$$
T_{X L}\left(E_{\gamma}\right)=2 \pi E_{\gamma}^{2 L+1} f_{X L}\left(E_{\gamma}\right)
$$

In general, the main contribution to statistical nuclear decay is from electric dipole $(E 1)$ and magnetic dipole $(M 1)$ transitions. Here, we assume only $E 1$ and $M 1$ dipole contributions to the photon strength. In addition the number of positive and negative parity states is taken as equal 


$$
\rho\left(E_{i}-E_{\gamma}, I_{f}, \pm \Pi_{f}\right)=\frac{1}{2} \rho\left(E_{i}-E_{\gamma}, I_{f}\right)
$$

Combining the $E 1$ and $M 1$ dipole strengths and the experimental $\gamma$-ray transmission coefficient in Eq. 6.5 yields

$$
B T\left(E_{\gamma}\right)=\left[f_{E 1}\left(E_{\gamma}\right)+f_{M 1}\left(E_{\gamma}\right)\right] E_{\gamma}^{3}
$$

where the slope of the experimental $\gamma$-ray transmission coefficient $T$ is already corrected. The parameter $B$ is determined from the average total radiative width of compound states $\left\langle\Gamma_{\gamma}\right\rangle$. The experimental $\left\langle\Gamma_{\gamma}\right\rangle$ with excitation energy $E$, spin $I$, and parity $\Pi$ can be written in terms of $f_{X L}\left(E_{\gamma}\right)[35]$

$$
\left\langle\Gamma_{\gamma}(E, I, \Pi)\right\rangle=\frac{1}{\rho(E, I, \Pi)} \sum_{X L} \sum_{I_{f}, \Pi_{f}} \int_{E_{\gamma}=0}^{E} d E_{\gamma} E_{\gamma}^{2 L+1} f_{X L}\left(E_{\gamma}\right) \rho\left(E-E_{\gamma}, I_{f}, \Pi_{f}\right)
$$

The summations and integration are over all final levels with spin $I_{f}$ and parity $\Pi_{f}$ which are accessible by $\gamma$ radiation with energy $E_{\gamma}$, and multipole type $X L$. The average total radiative width of neutron $s$-wave capture resonances with spins $I_{f} \pm 1 / 2$ can be written by combining the two assumptions given in Eqs. 6.6 and 6.7 in the general expression Eq. 6.8. This gives

$$
\begin{aligned}
\left\langle\Gamma_{\gamma}\left(B_{n}, I_{t} \pm 1 / 2, \Pi_{t}\right)\right\rangle= & \frac{1}{2 \rho\left(B_{n}, I_{t} \pm 1 / 2, \Pi_{t}\right)} \int_{E_{\gamma}=0}^{B_{n}} d E_{\gamma} B T\left(E_{\gamma}\right) \rho\left(B_{n}-E_{\gamma}\right) \\
& \times \sum_{J=-1}^{1} g\left(B_{n}-E_{\gamma} I_{t} \pm 1 / 2+J\right)
\end{aligned}
$$

where $I_{t}$ and $\Pi_{t}$ are the spin and parity of the target nucleus in the $(n, \gamma)$ reaction, and $\rho$ is the experimental level density. Furthermore, the level density is expressed as the product 
of the total level density and a spin distribution factor $g$. The factor $g$ is given by [6]

$$
g(E, I)=\frac{2 I+1}{2 \sigma^{2}} \exp \left[-\frac{(I+1 / 2)^{2}}{2 \sigma^{2}}\right]
$$

and is normalized to $\sum_{I} g \approx 1 . \sigma$ is the spin-cutoff parameter. As mentioned earlier, $\gamma$ rays below $1.5 \mathrm{MeV}$ are removed from the primary $\gamma$-ray matrix. Therefore, the $\gamma$-ray transmission coefficients below $E_{\gamma}<1.5 \mathrm{MeV}$ and the level densities above $E_{\gamma}>B_{n}-1.5$ $\mathrm{MeV}$ are extrapolated using an exponential function. The data points between the arrows in Fig. 6.5 are used for the extrapolation. The contribution from the extrapolation to the total radiative width does not exceed $15 \%$ [53].

All of the parameters in Eq. 6.9 are known except the normalization constant $B$ for ${ }^{57} \mathrm{Fe}$. Thus we can easily find $B$. The parameter $\left\langle\Gamma\left(E_{\gamma}\right)\right\rangle$ for ${ }^{57} \mathrm{Fe}$ is taken from Ref. [42]. Once the normalization constant $B$ is obtained, the sum of the electric and magnetic dipole strengths is determined from Eq. 6.7. Radiative strength functions for ${ }^{56} \mathrm{Fe}$ and ${ }^{57} \mathrm{Fe}$ are shown in Figs. 6.6 and 6.7, respectively. Note that the $\gamma$-ray strength function in ${ }^{56} \mathrm{Fe}$ is given in arbitrary units; the slope is correct, but the magnitude remains undetermined. The radiative strength function (RSF) increases with increasing $\gamma$-ray energy above $E_{\gamma}$ $=4.5 \mathrm{MeV}$ in ${ }^{56} \mathrm{Fe}$, as observed in rare-earth nuclei $[51,52,53]$. The $\mathrm{RSF}$ for ${ }^{57} \mathrm{Fe}$ reveals a rather flat structure above $E_{\gamma}=4 \mathrm{MeV}$. In general, the RSF is expected to decrease with decreasing $\gamma$-ray energies $[51,52,53]$. In ${ }^{56} \mathrm{Fe}$, the radiative strength has a minimum at around $4.5 \mathrm{MeV}$ energy, and increases with decreasing $\gamma$-ray energy. A similar enhancement at low energies is observed in ${ }^{57} \mathrm{Fe}$. We do not believe that this is an artifact of the data reduction since the same behavior was observed in ${ }^{27,28} \mathrm{Si}$ (see below). The RSF might depend, in addition to $E_{\gamma}$, on another parameter of a different origin, such as temperature.

The experimental RSFs are compared with model calculations. Again for the model calculations we assume that the $\gamma$-ray strength is dominated by dipole transitions. For the $E 1$ strength, we use two different descriptions: the Lorentzian giant electric dipole 


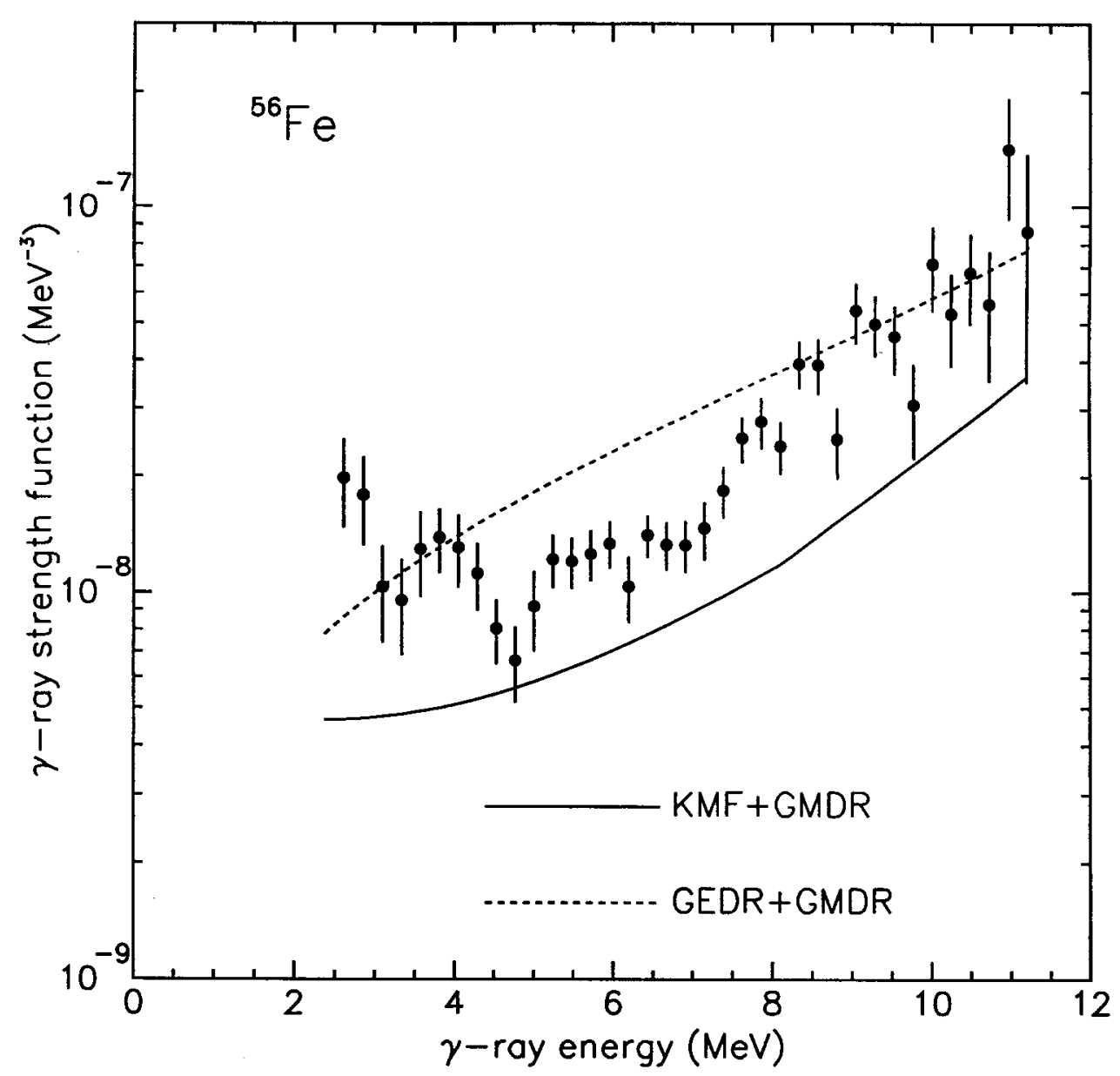

Figure 6.6: Radiative strength function for ${ }^{56} \mathrm{Fe}$. The experimental RSF (data points) is given in arbitrary units. The models KMF+GMDR and GEDR+GMDR are in absolute units, see text for details.

resonance (GEDR) model, and the Kadmenskiř-Markushev-Furman (KMF) model [38]. The first model is based on Brink's hypothesis, which assumes that giant resonances built on the ground state as well as those built on any excited state have the same size and shape, and the GEDR is expressed as

$$
f_{E 1}\left(E_{\gamma}\right)=\frac{1}{3 \pi^{2} \hbar^{2} c^{2}} \frac{\sigma_{E 1} E_{\gamma} \Gamma_{E 1}^{2}}{\left(E_{\gamma}^{2}-E_{E 1}^{2}\right)^{2}+E_{\gamma}^{2} \Gamma_{E 1}^{2}}
$$




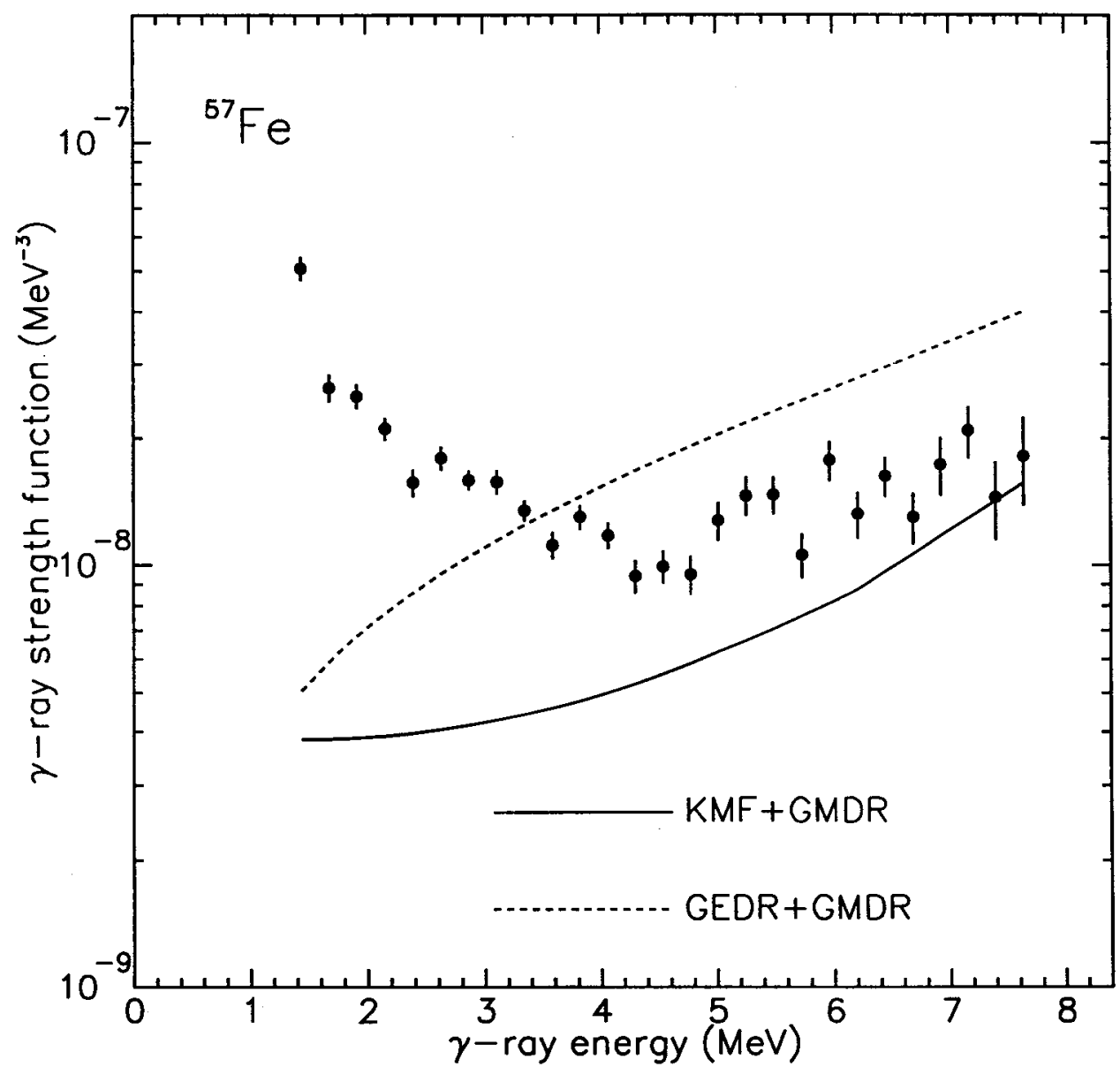

Figure 6.7: Radiative strength function for ${ }^{57} \mathrm{Fe}$.

where $\sigma_{E 1}, \Gamma_{E 1}$, and $E_{E 1}$ are the standard giant electric dipole resonance parameters derived from photoabsorption experiments.

In the KMF model [38], the Lorentzian expression is modified in order to reproduce the nonvanishing tail of the GEDR as $E_{\gamma} \rightarrow 0$ by introducing a temperature dependent width of the GEDR. This model is given by

$$
f_{E 1}\left(E_{\gamma}\right)=\frac{1}{3 \pi^{2} \hbar^{2} c^{2}} \frac{0.7 \sigma_{E 1} \Gamma_{E 1}^{2}\left(E_{\gamma}^{2}+4 \pi^{2} T^{2}\right)}{E_{E 1}\left(E_{\gamma}^{2}-E_{E 1}^{2}\right)^{2}}
$$


where $T=\sqrt{\left(B_{n}-E_{\gamma}-E_{1}\right) / a}$ is the nuclear temperature in the case of thermal neutron capture, $B_{n}$ represents the neutron binding energy, $a$ is the Fermi gas level density parameter, and $E_{1}$ is the back-shift parameter. The energy and temperature dependent width of the GEDR in this model is expressed by

$$
\Gamma_{E 1}\left(E_{\gamma}, T\right)=\frac{\Gamma_{E 1}}{E_{E 1}^{2}}\left(E_{\gamma}^{2}+4 \pi^{2} T^{2}\right)
$$

For the $M 1$ strength, we use a Lorentzian model based on the existence of the $M 1$ giant dipole resonance (GMDR), which is assumed to be related to shell-model spin-flip transitions between $l \pm \frac{1}{2}$ single-particle states [72]

$$
f_{M 1}\left(E_{\gamma}\right)=\frac{1}{3 \pi^{2} \hbar^{2} c^{2}} \frac{\sigma_{M 1} E_{\gamma} \Gamma_{M 1}^{2}}{\left(E_{\gamma}^{2}-E_{M 1}^{2}\right)^{2}+E_{\gamma}^{2} \Gamma_{M 1}^{2}} .
$$

In this expression, the Lorentzian damping width is described as independent of photon energy and temperature.

The GEDR and GMDR parameters are taken from the global parameterization of Ref. [42] for spherical nuclei $(A>50)$. The temperature in Eq. 6.12 is calculated from $T(\mathrm{MeV})=\sqrt{\left(B_{n}-2-E_{\gamma}-E_{1}\right) / a}$. In this expression $B_{n}-2 \mathrm{MeV}$ gives the average excitation energy from which primary $\gamma$ rays are emitted since an excitation-energy region $B_{n}-4 \mathrm{MeV}$ was used in extracting the level density and RSF. The resonance peak cross section $\sigma_{M 1}$ is adjusted in order to produce $f_{E 1} / f_{M 1}=0.0588 A^{0.878}$ at $E_{\gamma}=7 \mathrm{MeV}$.

The calculated $E 1$ and $M 1$ strengths are summed together for comparison with the experimental data. These models using different descriptions for the $E 1$ strength are denoted by GEDR+GMDR and KMF+GMDR in Figs. 6.6 and 6.7. The average contribution of the GMDR strength to the total strength over all the $\gamma$-ray energies is approximately $20 \%$. The slope of the RSF for both ${ }^{56} \mathrm{Fe}$ and ${ }^{57} \mathrm{Fe}$ is reproduced with the $\mathrm{KMF}+\mathrm{GMDR}$ models above $E_{\gamma}=4.5 \mathrm{MeV}$, as well as with the GEDR+GMDR models. Below $E_{\gamma}=4.5 \mathrm{MeV}$, the KMF model tends to follow the data better. Although the KMF 
model predicts the finite value of the RSF as $E_{\gamma} \rightarrow 0$, it does not reproduce the upward bend at low $E_{\gamma}[38]$.

A similar enhancement at low $E_{\gamma}$ is also observed in ${ }^{27,28} \mathrm{Si}[75]$ and ${ }^{96,97} \mathrm{Mo}$ isotopes [76]. For ${ }^{28} \mathrm{Si}$ levels, level lifetimes, and $\gamma$-decay branching ratios are known up to $E_{x}=9.6 \mathrm{MeV}$ from discrete $\gamma$-ray spectroscopy. The $\gamma$-ray transmission coefficient for ${ }^{28} \mathrm{Si}$ was calculated from the known lifetimes, and compared with the experimental data [75]. Surprising agreement between the experimental and calculated values of the $\gamma$-ray transmission coefficient leads us to speculate that the low-energy decay strength in medium-weight and light nuclei is anomalously stronger than the corresponding strength in the heavy nuclei.

The presence of this anomaly in the decay strength can be investigated by extracting the multiplicity of the $\gamma$ rays from different excitation energies. This has not yet been done. If the multiplicity of the $\gamma$ rays is high, this would provide more confidence that $\gamma$ rays with low energies are preferred in the decay scheme, and that the observed effect is not an artifact of the subtraction method.

\subsection{Level Densities and $\gamma$-ray Strength Functions for Rare-Earth Nuclei}

The method to extract the level density and $\gamma$-ray strength function was first applied to rare-earth nuclei. These heavy nuclei are deformed and their level density is relatively higher.

The level densities for ${ }^{148,149} \mathrm{Sm}(Z=62)[52],{ }^{161,162} \mathrm{Dy}(Z=66)[77],{ }^{166,167} \operatorname{Er}(Z$ $=68)[51]$, and ${ }^{171,172} \mathrm{Yb}(Z=70)[77,54]$ isotopes are shown in Fig. 6.8. The experiments with these rare-earth nuclei were performed for a longer period of time, and thus have much better statistics than the present experiment. Therefore, the level density was extracted per $120-\mathrm{keV}$ bin as opposed to $238 \mathrm{-keV}$ bin for the iron isotopes.

The level density in these rare-earth nuclei is higher than the level density in the iron isotopes by a factor of three orders of magnitude. This is not surprising: the mass number of the rare-earth nuclei is about three times the mass of the iron isotopes. Thus, the large number of particles opens more degrees of freedom for the nucleus for a given excitation 


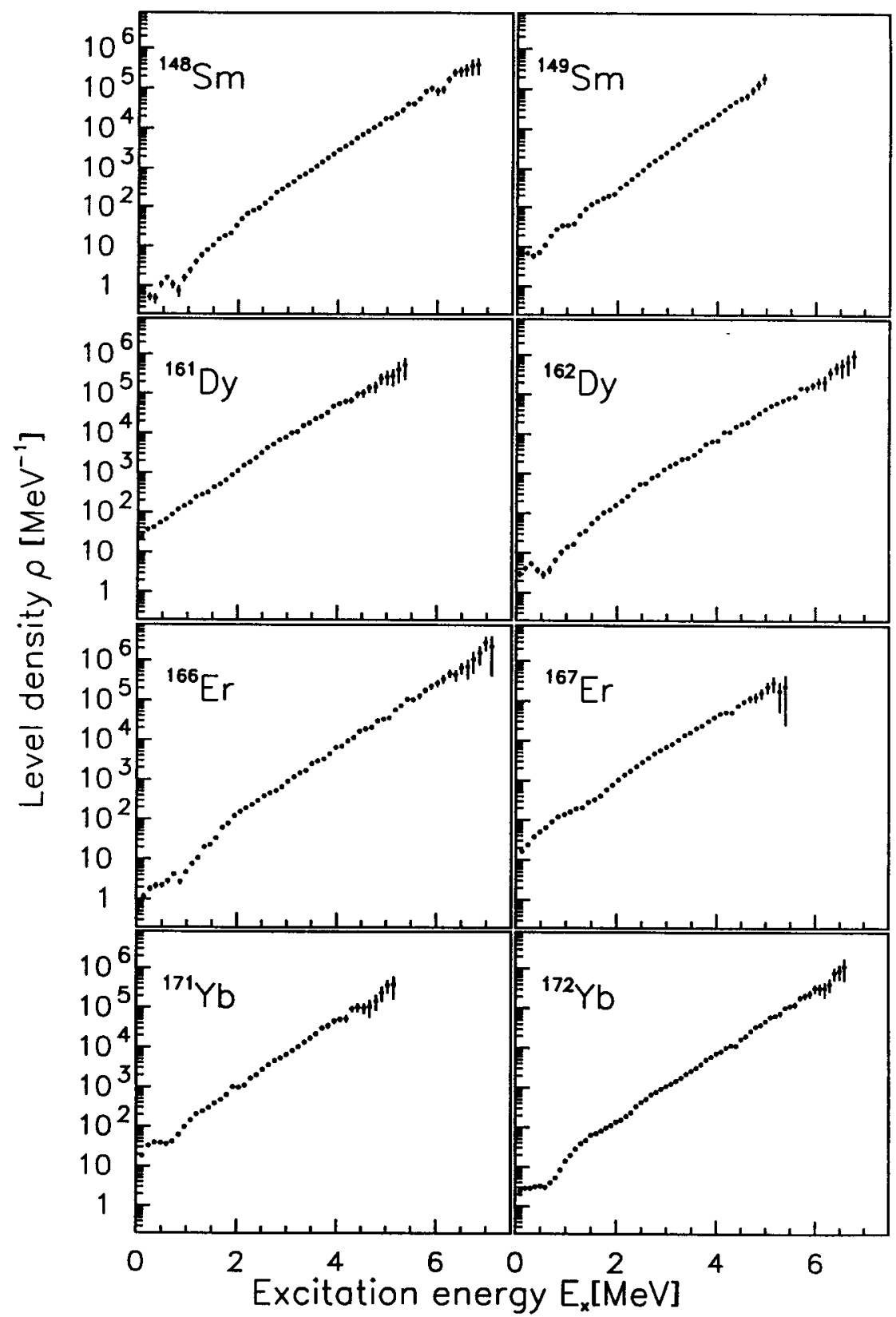

Figure 6.8: Level densities for rare-earth nuclei.

energy. It has been shown from the systematics [72] that the level density parameter $a$ is proportional to the mass number. The level density parameter essentially determines the slope of the level density. Therefore, changing this parameter from $a \approx 7 \mathrm{MeV}^{-1}$ for the mass $A=50-60$ region to $a \approx 20 \mathrm{MeV}^{-1}$ for the mass $A=160-170$ region results 
in a three-orders-of-magnitude increase in the level density. In addition, the level density depends on shell effects and odd or even number of particles.

Similar step-like structures are observed in these heavy nuclei. At low excitation energies, discrete structures are prominent, particularly in the even-even rare-earth nuclei. The density of levels increases more smoothly at higher excitations.

The radiative strength functions for these rare-earth nuclei are shown in Fig. 6.9. A common feature in all of these nuclei is that the radiative strength increases as the $\gamma$-ray energy increases. The enhancement observed at the low $\gamma$-ray energies in light and medium-weight nuclei is not observed in these heavy nuclei. Another common feature in Fig. 6.9 is the bump at low $\gamma$-ray energies. This bump is shifted to higher energies with increasing mass number. In fact, previous work [46] shows that the energy of the bump increases with neutron number in the $N=82-126$ region. This anomalous structure was observed in the $\gamma$-ray spectra of the $(n, \gamma)$ and $(d, p \gamma)$ reactions at low energies $[46,47,48$, $49,50]$. The bump is called the pygmy resonance due to its considerably lower strength compared to the GEDR. The pygmy resonance was first interpreted as the enhancement of the $E 1$ strength function [46]. However, a possible $M 1$ character (scissors mode) connected to orbital $M 1$ strength cannot be ruled out. A two-step cascade $(n, 2 \gamma)$ experiment was performed in order to determine the multipolarity of this resonance in ${ }^{172} \mathrm{Yb}$. In such an experiment, the spin and parity of the initial state is known. The two-step $\gamma$ rays decay into a low-lying state with an opposite parity to that of the initial state. These $\gamma$ rays are recorded in coincidence by requiring that the sum of the energy of the two $\gamma$ rays corresponds to the difference between the neutron binding energy and the energy of the chosen final state. Therefore, the deexcitation by two $\gamma$-ray transitions has to involve one electric- and one magnetic-dipole transition. The intensity and the spectral distribution of these two $\gamma$-ray transitions then provide an answer, after comparison with model calculations, whether the resonance structure at low frequencies is of electric- or magnetic-dipole character. The data analysis of this experiment continues. 


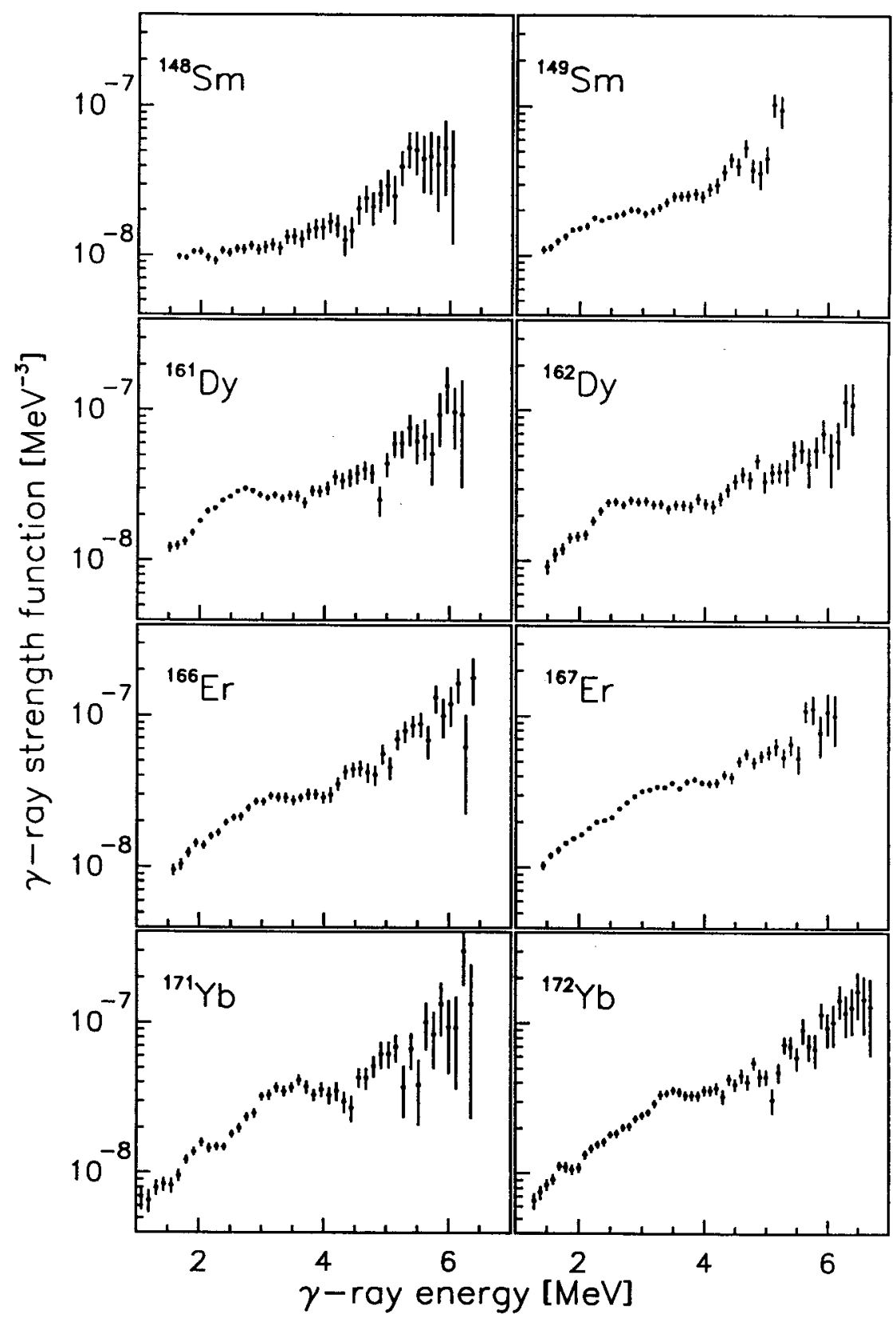

Figure 6.9: $\gamma$-ray strength functions for rare-earth nuclei.

\subsection{Thermodynamic Properties}

The concept of temperature is used to characterize a system in equilibrium. Other features include the energy and the density of states. In general temperatures are measured by comparing the system temperature to temperature standards by bringing the system 
into thermal contact with a thermometer. The thermometer is small and does not perturb the macroscopic system significantly. Such a procedure is not applicable for nuclear systems, which have a very small size. For nuclear systems, information on temperature is conveyed through the emission of particles after the system reaches its equilibrium. An annual review by Morrissey et al. [78] focuses on some methods of nuclear temperature measurements in nuclear reactions. These experimental methods for temperature determination involve examination of the kinetic energy spectra of emitted particles, their relative number, and their excited state population.

Here we do not measure the temperature of the nucleus, but instead measure the multiplicity of states $\Omega(E)$, which is proportional to the experimental level density $\rho(E)$. Then we study how the nucleus behaves if it is connected to a heat reservoir with a temperature $T$ using thermodynamic equations. The relationship between the temperature and the excitation energy of the system can be investigated using either the caloric curves derived within the statistical ensembles or the probability density function.

\subsubsection{Caloric Curves in Microcanonical and Canonical Ensembles}

Depending on the system under study, one can chose among different kinds of statistical ensembles in order to derive thermodynamic quantities. These ensembles are discussed in Chapter 2. The thermodynamic quantities derived within different ensembles give the same results in the thermodynamic limit. On the other hand, the choice of a specific ensemble may change results significantly for small systems. For example, the caloric curves derived within the microcanonical and canonical ensembles coincide for large systems; but the two caloric curves depart from each other for small systems. This fact is illustrated with a simple model in Appendix D.

The microcanonical ensemble is usually considered to be well-suited for atomic nuclei. Since the nuclear force has a very short range and a nucleus normally does not share its excitation energy with its external environment, a nucleus is usually considered as an isolated system. However, some thermodynamic quantities such as temperatures and heat capacities may have large fluctuations and negative values when derived within the micro- 


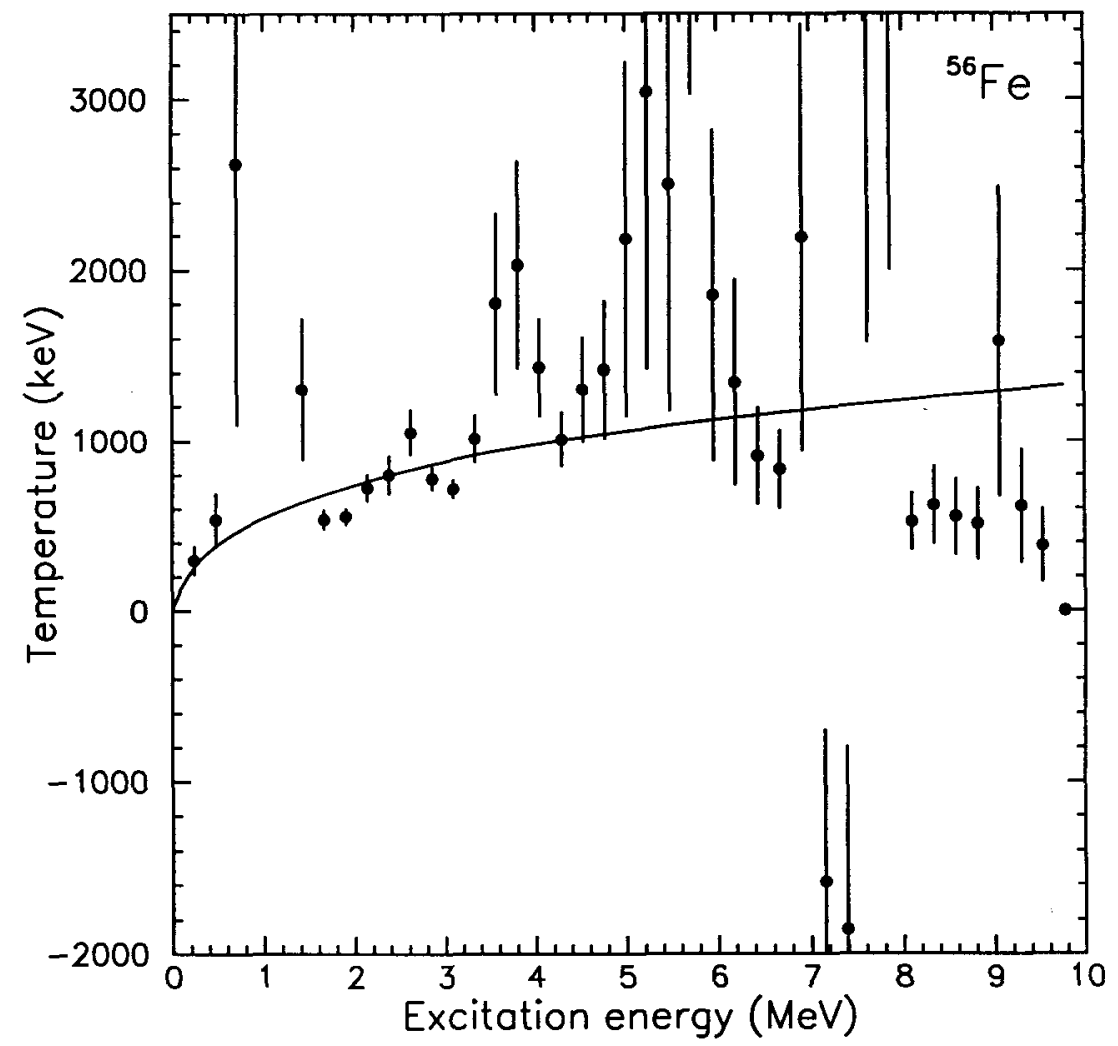

Figure 6.10: Experimental caloric curves for ${ }^{56} \mathrm{Fe}$ derived within the microcanonical (data points) and canonical (solid line) ensemble.

canonical ensemble (e.g. Refs. [51, 52]). This is also true for ${ }^{56,57} \mathrm{Fe}$, as is shown below. On the other hand, the canonical ensemble averages too much over structural changes of the system. Therefore, it is difficult to chose an appropriate ensemble for a small system. Here, we use both the microcanonical and canonical ensembles to derive the caloric curves in ${ }^{56} \mathrm{Fe}$ and ${ }^{57} \mathrm{Fe}$ and compare the results derived within both ensembles.

The caloric curves for ${ }^{56} \mathrm{Fe}$ and ${ }^{57} \mathrm{Fe}$ are shown in Figs. 6.10 and 6.11, respectively. The data points represent the microcanonical caloric curve (MCC), and the solid line represents the canonical caloric curve (CCC). 


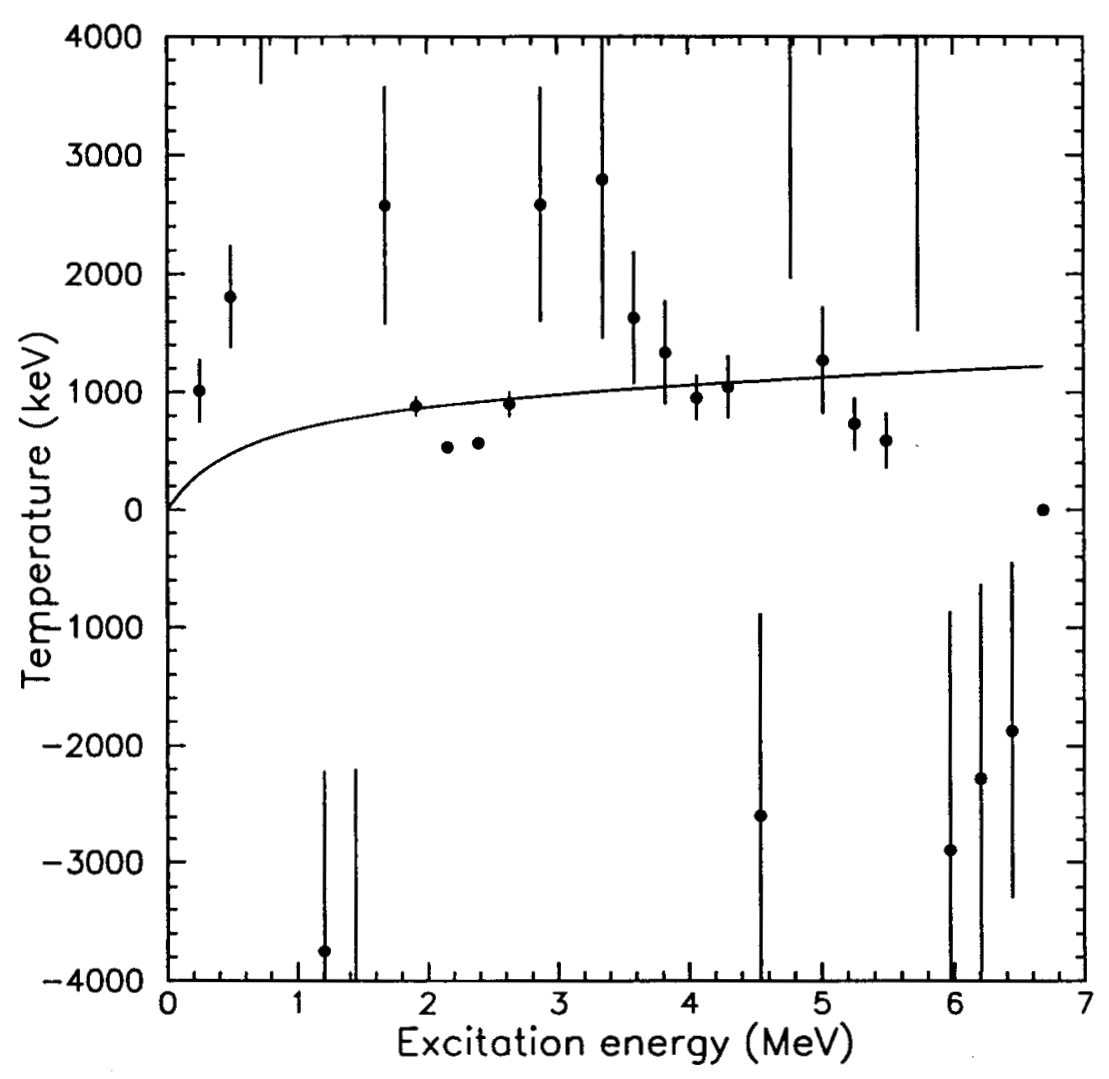

Figure 6.11: Same as in Fig. 6.10 except for ${ }^{57}$ Fe.

The MCC is defined as

$$
T=\left(\frac{\partial S}{\partial E}\right)^{-1}
$$

The entropy is given by $S=\ln \Omega(E)=\ln \rho(E)+S_{0}$, where the Boltzmann constant is set to unity for simplicity. The normalization constant $S_{0}$ can be adjusted to satisfy the third law of thermodynamics: when the temperature $T \rightarrow 0$, the entropy $S \rightarrow 0$. The constant $S_{0}$ is not important in this discussion since it vanishes when taking the derivative of the entropy. Small changes in the entropy give rise to large contributions and negative values in the temperature $T$ due to the differentiation. In order to reduce this sensitivity, the differentiation of $S$ is performed by a least $\chi^{2}$ fit of a quadratic polynomial to five 
adjacent data points at a time. Thus, this procedure smoothes an approximately $1.0-\mathrm{MeV}$ range. Since the particle-energy resolution is around $0.4 \mathrm{MeV}$, the differentiation removes some potential information. The error bars in MCC should not be considered as statistical errors since they reflect the fluctuations caused by the differentiation of the entropy $S$.

The caloric curve in the canonical ensemble is expressed as

$$
E=T^{2} \frac{\partial \ln Z}{\partial T}
$$

where the partition function in the canonical ensemble is given by

$$
Z(T)=\int_{0}^{\infty} \Omega(E) \exp (-E / T) d E
$$

The partition function is simply the Laplace transform of the multiplicity of states $\Omega(E)=$ $\Delta E \rho(E)$, where $\rho(E)$ is the level density of accessible levels at the energy $E$ given in energy bins $\Delta E$. Application of the Laplace transform in Eq. 6.17 smoothes much of the structure contained in the microcanonical level density (see Figs. 6.10 and 6.11). This significant averaging over structural changes in the canonical ensemble is caused by the integration in the calculation of the partition function. This smoothing effect can be quantified for the thermal average excitation energy, which is given by

$$
\langle E(T)\rangle=\frac{1}{Z} \int_{0}^{\infty} E \Omega(E) \exp (-E / T) d E
$$

using the standard deviation $\sigma_{E}=\sqrt{\left\langle E^{2}\right\rangle-\langle E\rangle^{2}}$. The standard deviation gives $\sigma_{E}=5$ $\mathrm{MeV}$ at $\langle E\rangle=6 \mathrm{MeV}$. Thus, the structures observed in the thermodynamic observables in the microcanonical ensemble are not apparent in the canonical ensemble.

We should also note that the integral in the partition function is defined from zero to infinity. However, our experimental level density goes up to around the neutron binding 
energy. Therefore, the level density is extrapolated from about the neutron binding energy up to $150-\mathrm{MeV}$ excitation energy using the Fermi gas model. The $150-\mathrm{MeV}$ upper limit is sufficiently high for the integrand to vanish for small temperatures.

In Figs. 6.10 and 6.11, it is clear that the MCC lies mostly above the CCC (note that the error bars in the MCC reflect the fluctuations of the differentiation.) The statistical errors in the CCC are very small due to the Laplace transform of $\Omega(E)$.

The CCCs for ${ }^{56} \mathrm{Fe}$ and ${ }^{57} \mathrm{Fe}$ are shown in Fig. 6.12 for comparison. The temperature $T(E)$ curve for ${ }^{57} \mathrm{Fe}$ is approximately $10 \%$ higher over all the energies than the temperature in ${ }^{56} \mathrm{Fe}$. We employ the CCCs when deriving the heat capacity in these nuclei.

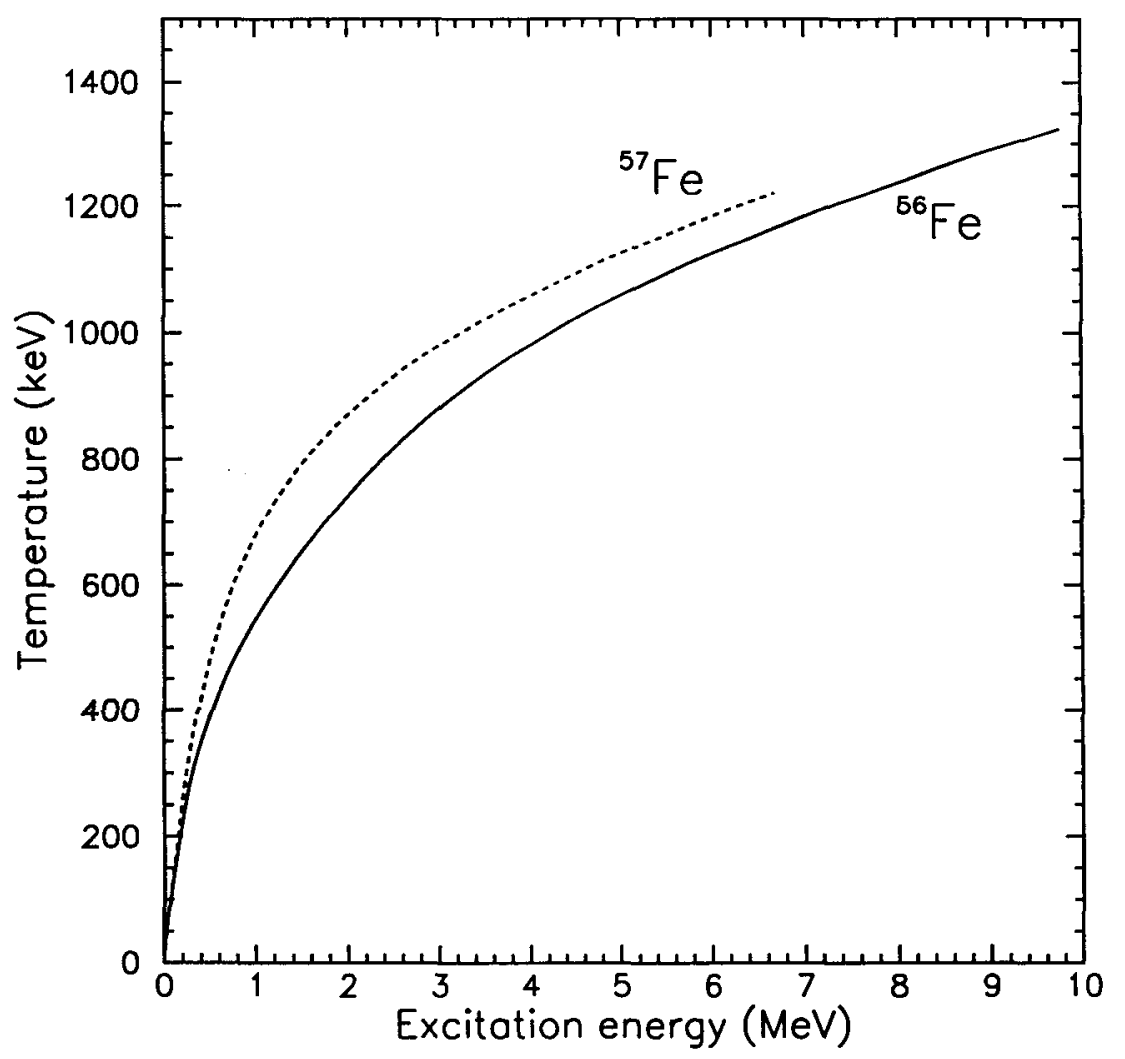

Figure 6.12: Canonical caloric curves for ${ }^{56,57} \mathrm{Fe}$.

The caloric curves for small systems are only approximations because the energy of the system is quite uncertain for a given temperature (see the next section). Furthermore, 
the caloric curves for small systems do not always give the most probable values of the system, but sometimes give the least probable values. This is illustrated in Appendix D first for a simple example, and then for ${ }^{56} \mathrm{Fe}$.

\subsubsection{Probability Density Function}

Another way of studying the energy-temperature relation of a system is the probability density function (PDF), which is the probability of the system having energy $E$ for a given temperature $T$, and is given by

$$
P(E)=\frac{\Omega(E) \exp (-E / T)}{Z(T)}
$$

The multiplicity of states $\Omega(E)$ is an increasing function of energy $E$, and $\exp (-E / T)$ is an exponentially decreasing function of $E$. Thus the product $\Omega(E) \exp (-E / T)$ has a maximum. In the thermodynamic limit, or for large systems, this product is very sharp - similar to a $\delta$ function. However, for small systems the multiplicity of states is much smaller, which makes the probability distribution broader, as shown in Fig. 6.13 for ${ }^{56} \mathrm{Fe}$.

In Fig. 6.13 the PDF for ${ }^{56} \mathrm{Fe}$ is shown for temperatures $T=1 \mathrm{MeV}$ and $T=1.5$ $\mathrm{MeV}$. The solid line separates two regions where the experimental and the extrapolated level density are used in the calculation of the probability $P(E)$. The data region shows the dynamics of the level density, while the extrapolation region does not show the same structure since the Fermi-gas level density is a smooth function of energy. Clearly, the probability distribution is mostly weighted by the experimental data for $T<1 \mathrm{MeV}$. For increasing temperatures, the energy of the system increases; therefore, the PDF depends more and more on the extrapolated level density.

In general caloric curves are constructed by replacing the distribution of energies (temperatures) for a given temperature (energy) by the most probable value. For large systems this procedure gives satisfactory results. However, for small systems the energy of the system has a broad distribution for a given temperature and the probability distribution may have more than one maximum for a given temperature. Therefore the caloric 


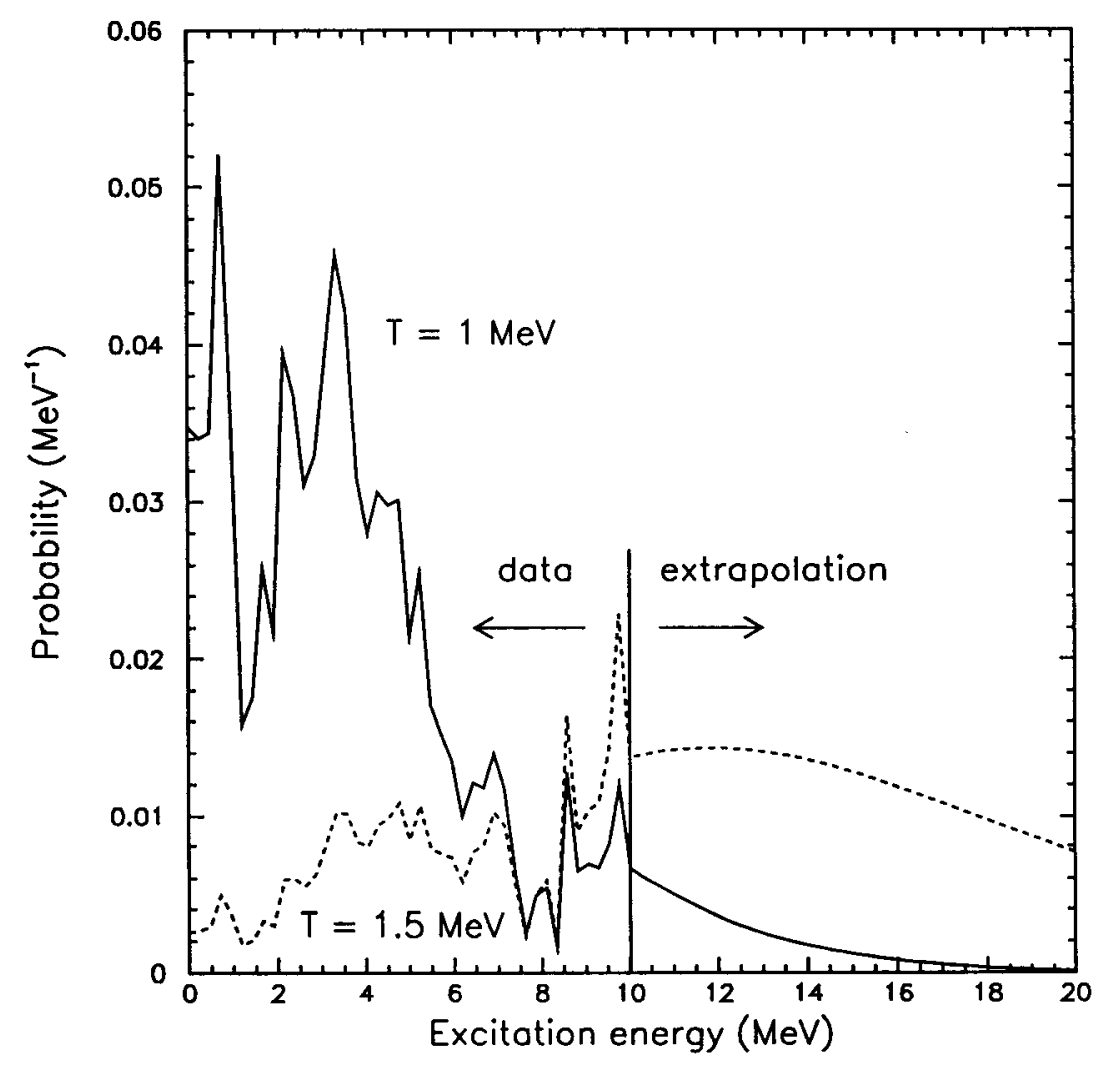

Figure 6.13: Probability density function for ${ }^{56} \mathrm{Fe}$.

curves for small systems are only approximations. The caloric curves for small and large systems are compared in Appendix D using a two-dimensional $P(E, T)$ distribution.

\subsubsection{Heat Capacity}

The nuclear heat capacity is a measure of the degrees of freedom. It is given by

$$
C_{V}=\left(\frac{\partial E}{\partial T}\right)_{V}
$$

where $E$ is the average energy of the system determined by the caloric curves (see Fig. 6.12). Since double differentiation of the entropy in the microcanonical ensemble introduces large fluctuations in the heat capacity, the nuclear heat capacity is derived only 


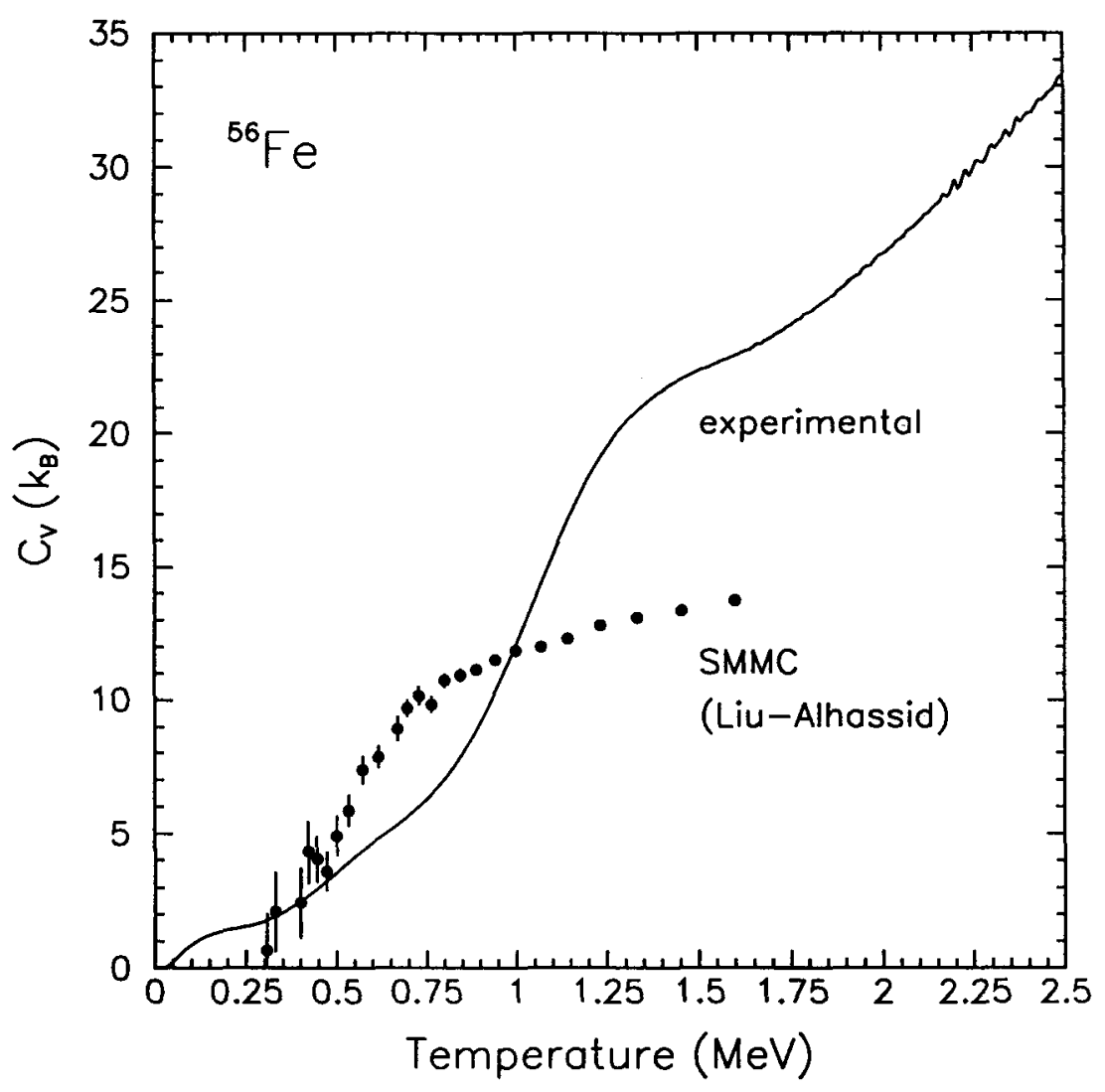

Figure 6.14: Experimental and theoretical heat capacities for ${ }^{56}$ Fe derived within the canonical ensemble.

using the canonical ensemble. The heat capacity in several iron isotopes has been calculated by Liu and Alhassid using the shell model Monte Carlo (SMMC) approach with a complete $\left(p f+0 g_{9 / 2}\right)$ shell [23]. In Figs. 6.14 and 6.15 the theoretical and experimental heat capacities are plotted for ${ }^{56} \mathrm{Fe}$ and for ${ }^{57} \mathrm{Fe}$. However, one cannot directly compare the experimental and theoretical results, since the theoretical calculations were performed using the state density, and the experimental heat capacity is obtained using the level density. The relation between the state and the level density is given by

$$
W(E)=\sum_{J}(2 J+1) \rho(E, J)
$$




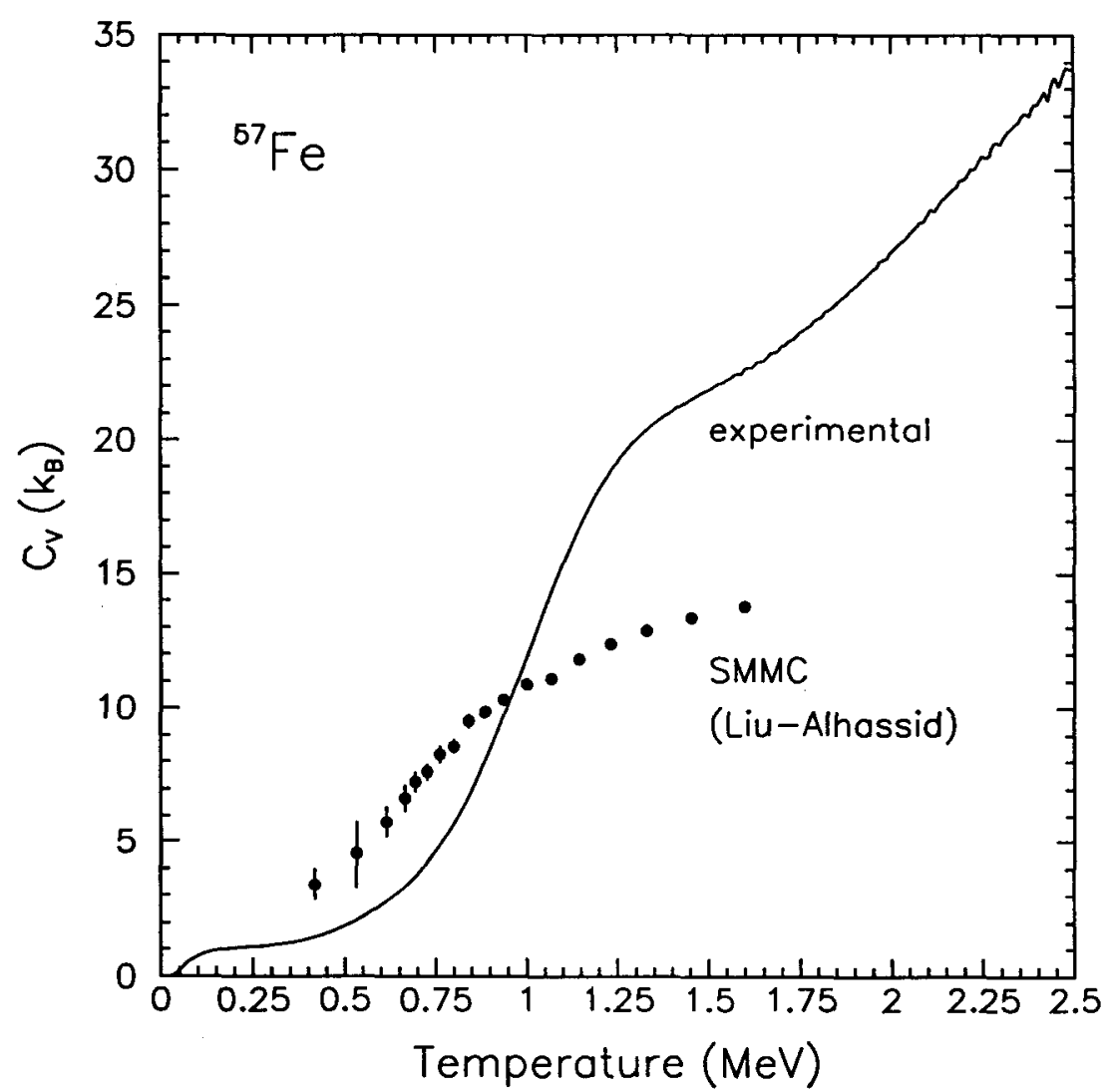

Figure 6.15: Experimental and theoretical heat capacities for ${ }^{57} \mathrm{Fe}$ derived within the canonical ensemble.

where $W(E)$ is the state density, which includes all of the magnetic substates. The theoretical and the experimental heat capacities have local enhancement at different temperatures, $0.7 \mathrm{MeV}$ and $1.3 \mathrm{MeV}$, respectively. The saturation above this enhancement in the model calculations is an artifact of the finite model space. Thus, as the temperature increases, the nucleons cannot be scattered across high energies since there are not enough single-particle levels to excite. Therefore, the heat capacity would eventually turn over and go to zero at high temperatures. This effect is known as the Schottky anomaly in the heat capacity [79]. In the new shell model Monte Carlo calculations, continuum contributions are taken into account and the Schottky anomaly disappears [80].

The difference between the experimental and theoretical heat capacities might be due to the uncertainty in the spin cut-off parameter which is used in the description of 
the density of levels of given angular momentum $J$ at a given energy. The spin cut-off parameter is not known.

The experimental heat capacity for ${ }^{56,57} \mathrm{Fe}$ exhibits an $S$ shape. This has been interpreted as a fingerprint of a second-order phase transition from a phase with strong pair correlations to a phase with weaker pairing correlations [81]. However, it is difficult to draw a quantitative conclusion. The shoulder in the theoretical heat capacity is also interpreted as a pairing transition [23]. Furthermore, the number of $J=0$ pairs were calculated as a function of temperature in SMMC. A rapid suppression of the number of neutron pairs was correlated with the shoulder observed in the heat capacity [23].

In Fig. 6.16 we show the sensitivity of the experimental heat capacity to the uncertainty in the slope of the level density. Since the level density at the neutron binding energy has an uncertainty (the level density for ${ }^{57} \mathrm{Fe}$ at $B_{n}$ is $1070 \pm 160 \mathrm{MeV}^{-1}$ ), we take the upper and lower uncertainty limit in calculating the experimental level density. Furthermore changing the lower excitation energy region in the normalization procedure affects the slope of the level density. By considering the uncertainties at $B_{n}$ and low excitationenergy region, we determine the level density with a maximum lower and higher slope. In Fig. 6.16 the heat capacities calculated using the extremal slopes of the lower and higher level density are shown in ${ }^{57} \mathrm{Fe}$. The change in the slope of the level density does not affect the heat capacity at low $(T<0.4 \mathrm{MeV})$ and at high $(T>2 \mathrm{MeV})$ temperatures. In between these temperatures the maximum change in the $S$ shape of the heat capacity does not exceed $5 \%$. 


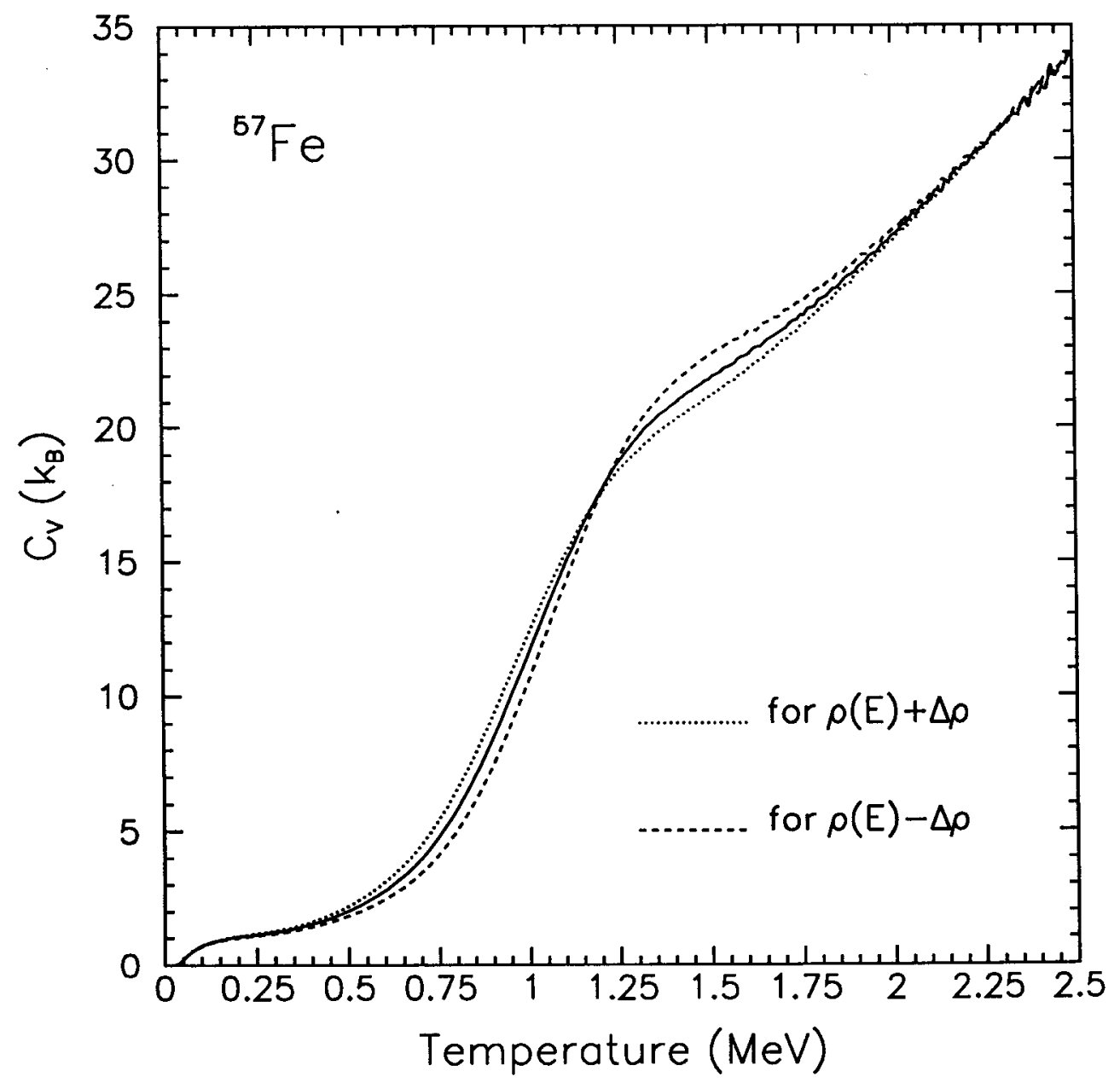

Figure 6.16: Sensitivity of the experimental canonical heat capacity in ${ }^{57} \mathrm{Fe}$ to the slope of the experimental level density. 


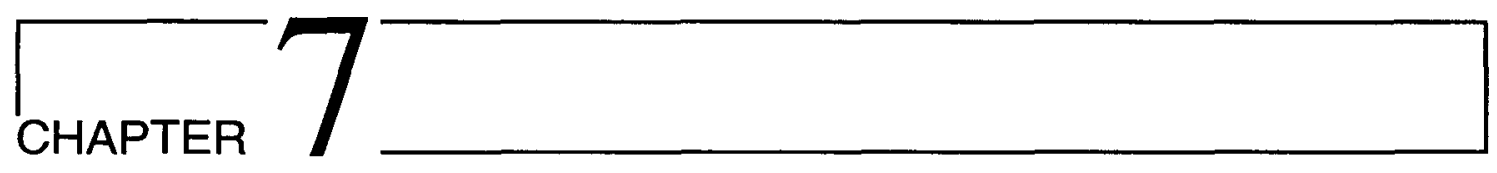

\section{Summary and Outlook}

\subsection{Summary and Conclusions}

Nuclear level densities and radiative strength functions play a crucial role for applied nuclear reaction model calculations. Level densities for many nuclei are known at low excitation energies from counting discrete levels, and at the neutron binding energy $B_{n}$ from counting neutron resonances. Between those energies the level density is usually obtained by interpolating experimental data using phenomenological models such as the Fermi-gas model.

A method developed by the Oslo Cyclotron group fills the gap between low and high excitation energies by extracting the level density from zero to close to the neutron binding energy. This extraction method relies on the primary $\gamma$-ray spectra, and also provides a simultaneous determination of the radiative strength function (RSF). The method has been applied to study several rare-earth nuclei, which are deformed and have high level densities. The method works well in the heavy-mass region.

The primary motivation of this dissertation was to apply and test the method in a lighter mass region where the level density is relatively low. We studied ${ }^{56} \mathrm{Fe}$ and ${ }^{57} \mathrm{Fe}$. The iron isotopes are of particular interest since they are the seed nuclei for the synthesis of the heavy elements by the $s$ and $r$ processes. In addition the heat capacity of several iron isotopes was recently calculated by Liu and Alhassid within the shell model Monte Carlo method; a signature of a pairing phase transition in the heat capacity was identified, 
and was correlated with the suppression of the number of spin-zero neutron pairs with increasing temperature.

The experimental primary $\gamma$-ray matrix for ${ }^{56} \mathrm{Fe}$ and ${ }^{57} \mathrm{Fe}$, which gives a primary $\gamma$-ray spectrum for each excitation-energy bin of the residual nucleus, is obtained using light-ion reactions. It is important that a large number of states of the residual nucleus is populated. This is accomplished by the ${ }^{57} \mathrm{Fe}\left({ }^{3} \mathrm{He}, \alpha \gamma\right)$ and ${ }^{57} \mathrm{Fe}\left({ }^{3} \mathrm{He},{ }^{3} \mathrm{He}^{\prime} \gamma\right)$ reactions in a single experiment. The $\left({ }^{3} \mathrm{He}, \alpha \gamma\right)$ reaction is particularly well suited since the wave function of the neutron hole state left in the target nucleus is fragmented over a large number of single-particle states. The $\gamma$ rays and particles were detected with the CACTUS detector array, which consists of $28 \mathrm{NaI}(\mathrm{Tl})$ detectors and $8 \mathrm{Si}(\mathrm{Li})$ particle telescopes. From recorded particle- $\gamma$ coincidences, the total $\gamma$-ray cascade spectrum is constructed with respect to the initial excitation energy of the residual nucleus. These spectra are unfolded using a Compton subtraction method. The primary $\gamma$-ray spectra are then obtained by applying a subtraction method, which subtracts the second- and higher-order $\gamma$ rays from the total $\gamma$-ray cascade spectrum of the corresponding excited state.

The experimental primary $\gamma$-ray matrix is the starting point to extract the level density and the radiative strength function. This matrix is factored according to the Axel-Brink hypothesis as a product of the level density $\rho$ at the final energy and the $\gamma$ ray transmission coefficient $T$. Neither $\rho$ nor $T$ is known a priori. There are an infinite number of solutions for this factorization. One of the solutions is found by applying a least $\chi^{2}$ fit to the primary $\gamma$-ray data through an iterative procedure. All of the solutions are related to each other by the multiplication of an exponential function to any arbitrary solution. Using this relation, the solution obtained from the $\chi^{2}$ fit is normalized to the known discrete states at low excitation energies, and to the neutron resonance data at the neutron binding energy. The radiative strength function normalization is performed using the average total radiative width of neutron resonances.

One of the most interesting findings of the present work is the step structure above the pairing gap in the level density curves in ${ }^{56,57} \mathrm{Fe}$. This step structure is interpreted as nucleon pair breaking. The energy that corresponds to the first pair breaking sheds light 
on the pairing gap and the single-particle level spacing. Breaking more and more nucleon Cooper pairs results in a large increase in the entropy. The step structures smooth out with increasing excitation energy.

Another common feature for ${ }^{56,57} \mathrm{Fe}$ is that the radiative strengths in both isotopes increase anomalously at low $\gamma$-ray energies. This behavior was not observed in heavy nuclei. The experimental strength functions are compared with giant dipole resonance models. However, the low-energy effect in the present work cannot be explained by the current phenomenological models. Similar enhancements at low $\gamma$-ray energies are also observed in ${ }^{27,28} \mathrm{Si}$ and ${ }^{96,97} \mathrm{Mo}$ isotopes in similar $\left({ }^{3} \mathrm{He}, \alpha \gamma\right)$ and $\left({ }^{3} \mathrm{He},{ }^{3} \mathrm{He}^{\prime} \gamma\right)$ reactions. The calculated radiative strength for ${ }^{28} \mathrm{Si}$ using the known level lifetime data for levels up to $10 \mathrm{MeV}$ agrees surprisingly well with the data from the Oslo Cyclotron Laboratory. Therefore we believe that this effect is real and is not an artifact of the data reduction, and that the low-energy strength is relatively stronger in light- and medium-mass nuclei than the corresponding strength in heavy nuclei. The origin of this enhancement remains unknown.

The nuclear level density is closely related to the thermodynamics of the nucleus. The level density extracted over a wide range of excitation energies provides information on the thermodynamics of ${ }^{56,57} \mathrm{Fe}$. We considered the energy-temperature relation in these isotopes using two different methods: the caloric curves and the probability density functions. We obtain the caloric curves in the microcanonical and canonical ensembles and show that the caloric curves for small systems are different. Using the probability density functions we show that the caloric curves cannot always describe a small system with the most probable energy for a given temperature, but sometimes describe the system with the locally least probable value.

The heat capacities for ${ }^{56,57} \mathrm{Fe}$ are obtained within the canonical ensemble. The $S$ shape of the heat capacity can be interpreted as a fingerprint of a second-order phase transition in these nuclei. The experimental heat capacities are also compared with theoretical heat capacities obtained in Shell Model Monte Carlo calculations. However, a direct comparison cannot be made since the experimental and theoretical heat capacities 
are derived using the level density and state density, respectively. The difference in the experimental and the theoretical values may be due to the uncertainty in the spin cutoff parameter.

\subsection{Outlook}

We would next like to investigate the presence of the anomaly in the low-energy decay strength by extracting the multiplicity of $\gamma$ rays from different excitation energies. If the multiplicity of the $\gamma$ rays is high, this will provide more confidence that $\gamma$ rays with low energies are preferred, and that this effect is not an artifact of the subtraction method.

The extraction method is based on the Axel-Brink hypothesis which uses an energyindependent width of the giant dipole resonance. The radiative strength functions are the subject of recent investigations. The latest phenomenological models treat the giant dipole resonance with an energy and temperature dependent width. More experimental data are needed to verify whether or not this description is correct. In that case the extraction method should be modified with an improved model of the radiative strength function. Improved understanding of the low-energy radiative strength function requires additional measurements in light- and medium-weight nuclei. 


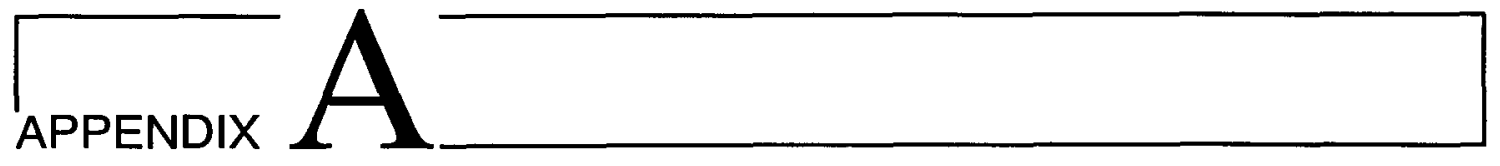

\section{Transformation Functions}

The experimental normalized primary $\gamma$-ray matrix $P\left(E_{i}, E_{\gamma}\right)$ provides the $\gamma$-ray decay probability distribution per excitation-energy bin. In Chapter 3 , the $P\left(E_{i}, E_{\gamma}\right)$ matrix is written as a product of the $\gamma$-ray transmission coefficient $T$ and the level density $\rho$ at the final excitation energy according to the Axel-Brink hypothesis

$$
P\left(E_{i}, E_{\gamma}\right) \propto T\left(E_{\gamma}\right) \rho\left(E_{i}-E_{\gamma}\right)
$$

or more explicitly after normalizing the right-hand side of Eq. A.1

$$
P\left(E_{i}, E_{\gamma}\right)=\frac{T\left(E_{\gamma}\right) \rho\left(E_{i}-E_{\gamma}\right)}{\sum_{E_{\gamma}^{\prime}=E_{\gamma}^{\text {min }}}^{E_{i}} T\left(E_{\gamma}^{\prime}\right) \rho\left(E_{i}-E_{\gamma}^{\prime}\right)}
$$

There are an infinite number of solutions to Eq. A.2. Here we will show that we can construct all of the solutions that describe the experimental $P\left(E_{i}, E_{\gamma}\right)$ matrix equally well from one arbitrary solution.

Consider $\rho\left(E_{i}-E_{\gamma}\right)$ and $T\left(E_{\gamma}\right)$ together as one solution. If $\tilde{\rho}\left(E_{i}-E_{\gamma}\right)$ and $\tilde{T}\left(E_{\gamma}\right)$ together are another solution given by 


$$
\tilde{\rho}\left(E_{i}-E_{\gamma}\right)=\rho\left(E_{i}-E_{\gamma}\right) g\left(E_{i}-E_{\gamma}\right)
$$

$$
\tilde{T}\left(E_{\gamma}\right)=T\left(E_{\gamma}\right) f\left(E_{\gamma}\right)
$$

then the new $\tilde{\rho}\left(E_{i}-E_{\gamma}\right)$ and $\tilde{T}\left(E_{\gamma}\right)$ should also satisfy Eq. A.2, i.e.,

$$
P\left(E_{i}, E_{\gamma}\right)=\frac{\tilde{T}\left(E_{\gamma}\right) \tilde{\rho}\left(E_{i}-E_{\gamma}\right)}{\sum_{E_{\gamma}^{\prime}=E_{\gamma}^{\min }}^{E_{1}} \tilde{T}\left(E_{\gamma}^{\prime}\right) \tilde{\rho}\left(E_{i}-E_{\gamma}^{\prime}\right)}
$$

Thus,

$$
\frac{T\left(E_{\gamma}\right) \rho\left(E_{i}-E_{\gamma}\right)}{\sum_{E_{\gamma}^{\prime}=E_{\gamma}^{\min }}^{E_{i}} T\left(E_{\gamma}^{\prime}\right) \rho\left(E_{i}-E_{\gamma}^{\prime}\right)}=\frac{\tilde{T}\left(E_{\gamma}\right) \tilde{\rho}\left(E_{i}-E_{\gamma}\right)}{\sum_{E_{\gamma}^{\prime}=E_{\gamma}^{\min }}^{E_{i}} \tilde{T}\left(E_{\gamma}^{\prime}\right) \tilde{\rho}\left(E_{i}-E_{\gamma}^{\prime}\right)}
$$

Substituting $\tilde{T}\left(E_{\gamma}\right)$ and $\tilde{\rho}\left(E_{i}-E_{\gamma}\right)$ in the last equation gives

$$
f\left(E_{\gamma}\right) g\left(E_{i}-E_{\gamma}\right) \sum_{E_{\gamma}^{\prime}=E_{\gamma}^{\min }}^{E_{i}} T\left(E_{\gamma}^{\prime}\right) \rho\left(E_{i}-E_{\gamma}^{\prime}\right)=\sum_{E_{\gamma}^{\prime}=E_{\gamma}^{\min }}^{E_{i}} T\left(E_{\gamma}^{\prime}\right) f\left(E_{\gamma}^{\prime}\right) \rho\left(E_{i}-E_{\gamma}^{\prime}\right) g\left(E_{i}-E_{\gamma}^{\prime}\right)
$$

Equation A.7 clearly shows that the product $f\left(E_{\gamma}\right) g\left(E_{i}-E_{\gamma}\right)$ is independent of $E_{\gamma}$, and depends only on $E_{i}$

$$
f\left(E_{\gamma}\right) g\left(E_{i}-E_{\gamma}\right)=h\left(E_{i}\right)
$$

The condition must hold for $E_{i}=E_{\gamma}$. If $g(0)=A$, then 


$$
A f\left(E_{\gamma}\right)=h\left(E_{\gamma}\right)
$$

Substituting this into Eq A.8 yields

$$
f\left(E_{\gamma}\right) g\left(E_{i}-E_{\gamma}\right)=A f\left(E_{i}\right)
$$

The condition must also hold for $E_{\gamma}=0$, with $f(0)=B$, then Eq. A.10 becomes

$$
B g\left(E_{i}\right)=A f\left(E_{i}\right)
$$

Inserting this in Eq. A.10, one gets

$$
g\left(E_{\gamma}\right) g\left(E_{i}-E_{\gamma}\right)=A g\left(E_{i}\right)
$$

The function $g$ can be expanded in a Taylor series in the limit of $E_{\gamma} \rightarrow 0$. By ignoring second and higher terms in the Taylor expansion

$$
\left(A+g^{\prime}(0) E_{\gamma}\right)\left(g\left(E_{i}\right)-g^{\prime}\left(E_{i}\right) E_{\gamma}\right)=A g\left(E_{i}\right)
$$

Again neglecting second-order terms in $E_{\gamma}$ gives

$$
A g^{\prime}\left(E_{i}\right)=g^{\prime}(0) g\left(E_{i}\right)
$$

The approximation in the limit of $E_{\gamma} \rightarrow 0$ is valid, because $g$ is a function of only one 
variable, and we let $g$ depend on $E_{i}$ instead of $E_{\gamma}$. We define $g^{\prime}(0) / A \equiv \alpha$, and write the differential equation

$$
\int_{0}^{E_{i}} \frac{d g\left(E_{i}\right)}{g\left(E_{i}\right)}=\alpha \int_{0}^{E_{i}} d E_{i} .
$$

Thus the function $g$ has an exponential form

$$
g\left(E_{i}\right)=A e^{\alpha E_{i}}
$$

By substituting $g$ into Eq. A.11, the other transformation function is obtained as

$$
f\left(E_{i}\right)=B e^{\alpha E_{i}}
$$




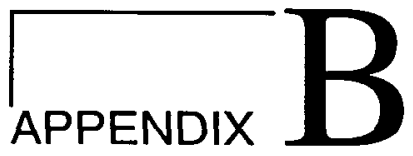

\section{$\chi^{2}$ Minimization}

In Chapter 3, the normalized experimental primary $\gamma$-ray matrix is factorized as a product of the $\gamma$-ray transmission coefficient $T$ and the level density $\rho$ at the final excitation energy as

$$
P\left(E_{i}, E_{\gamma}\right) \propto T\left(E_{\gamma}\right) \rho\left(E_{i}-E_{\gamma}\right)
$$

or more explicitly by normalizing the theoretical description of the $P\left(E_{i}, E_{\gamma}\right)$ matrix

$$
P\left(E_{i}, E_{\gamma}\right)=\frac{T\left(E_{\gamma}\right) \rho\left(E_{i}-E_{\gamma}\right)}{\sum_{E_{\gamma}^{\prime}=E_{\gamma}^{m i n}}^{E_{i n}} T\left(E_{\gamma}^{\prime}\right) \rho\left(E_{i}-E_{\gamma}^{\prime}\right)}
$$

Due to the structure of this equation, there are an infinite number of different solutions. In order to find one of the solutions, we apply a least $\chi^{2}$ method. In this method the reduced $\chi^{2}$ is minimized for $T\left(E_{\gamma}\right)$ at each $\gamma$-ray energy and $\rho\left(E_{i}-E_{\gamma}\right)$ at each excitation energy. The equations obtained from the $\chi^{2}$ minimization are not a linear system of equations. Therefore, they cannot be solved analytically. However, solving these equations numerically is tedious. Thus, an iteration procedure is employed. The iteration starts with arbitrary values of $T$ and $\rho$, and then calculates new estimates using the values from the 
previous iteration. Here, we derive the formulae by minimizing the $\chi^{2}$ that calculates the new estimates of $T$ and $\rho$. The iteration procedure is implemented in a FORTRAN program, and continues until the $\chi^{2}$ converges.

The reduced $\chi^{2}$ is defined as

$$
\chi^{2}=\frac{1}{N_{\text {free }}} \sum_{E_{i}=E_{i}^{\text {min }}}^{E_{i}^{\max }} \sum_{E_{\gamma}=E_{\gamma}^{\text {min }}}^{E_{i}}\left(\frac{P\left(E_{i}, E_{\gamma}\right)-P_{e x p}\left(E_{i}, E_{\gamma}\right)}{\triangle P_{e x p}\left(E_{i}, E_{\gamma}\right)}\right)^{2}
$$

If we substitute $P\left(E_{i}, E_{\gamma}\right)$ into Eq. B.3

$$
\chi^{2}=\frac{1}{N_{\text {free }}} \sum_{E_{i}=E_{i}^{\min }}^{E_{i}^{\max }} \sum_{E_{\gamma}=E_{\gamma}^{\min }}^{E_{i}}\left(\frac{\frac{T\left(E_{\gamma}\right) \rho\left(E_{i}-E_{\gamma}\right)}{\sum_{E_{\gamma}^{\prime}=E_{\gamma}^{\min } T\left(E_{\gamma}^{\prime}\right) \rho\left(E_{i}-E_{\gamma}^{\prime}\right)}-P_{\exp }\left(E_{i}, E_{\gamma}\right)}}{\triangle P_{\exp }\left(E_{i}, E_{\gamma}\right)}\right)^{2}
$$

$\chi^{2}$ is minimized with respect to $T\left(E_{\gamma}\right)$

$$
\frac{\partial}{\partial T\left(E_{\gamma}\right)} \chi^{2}=0
$$

or more explicitly

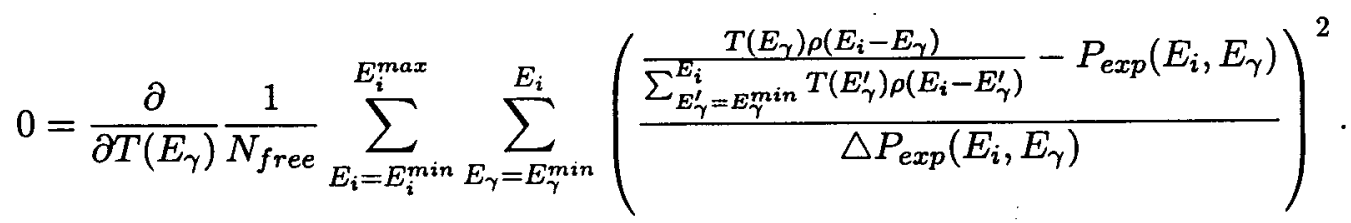

$N_{\text {free }}$ is a constant, and therefore, can be taken out of the expression. Taking the square of the parenthesis gives 


$$
\begin{aligned}
& 0=\frac{\partial}{\partial T\left(E_{\gamma}\right)}\left\{\sum_{E_{i}=E_{i}^{\min }}^{E_{i}^{\max }} \sum_{E_{\gamma}=E_{\gamma}^{\min }}^{E_{i}}\left[\frac{T\left(E_{\gamma}\right) \rho\left(E_{i}-E_{\gamma}\right)}{\triangle P_{e x p}\left(E_{i}, E_{\gamma}\right) \sum_{E_{\gamma}^{\prime}=E_{\gamma}^{\min }}^{E_{i}} T\left(E_{\gamma}^{\prime}\right) \rho\left(E_{i}-E_{\gamma}^{\prime}\right)}\right]^{2}\right. \\
& -2 \sum_{E_{i}=E_{i}^{\min }}^{E_{i}^{\max }} \sum_{E_{\gamma}=E_{\gamma}^{\min }}^{E_{i}} \frac{T\left(E_{\gamma}\right) \rho\left(E_{i}-E_{\gamma}\right) P_{e x p}\left(E_{i}, E_{\gamma}\right)}{\left[\triangle P_{e x p}\left(E_{i}, E_{\gamma}\right)\right]^{2} \sum_{E_{\gamma}^{\prime}=E_{\gamma}^{\min }}^{E_{i}} T\left(E_{\gamma}^{\prime}\right) \rho\left(E_{i}-E_{\gamma}^{\prime}\right)} \\
& \left.+\sum_{E_{i}=E_{i}^{\min }}^{E_{i}^{\max }} \sum_{E_{\gamma}=E_{\gamma}^{\min }}^{E_{i}}\left[\frac{P_{\exp }\left(E_{i}, E_{\gamma}\right)}{\triangle P_{e x p}\left(E_{i}, E_{\gamma}\right)}\right]^{2}\right\} \text {. }
\end{aligned}
$$

Since the last term of Eq. B.7 is independent of $T$, its derivative is zero. Ordering the other sums yields

$$
\begin{aligned}
& 0=\frac{\partial}{\partial T\left(E_{\gamma}\right)}\left\{\sum_{E_{i}=E_{i}^{\min }}^{E_{i}^{\max }} \frac{1}{\left[\sum_{E_{\gamma}^{\prime}=E_{\gamma}^{m i n}}^{E_{i}} T\left(E_{\gamma}^{\prime}\right) \rho\left(E_{i}-E_{\gamma}^{\prime}\right)\right]^{2}} \sum_{E_{\gamma}=E_{\gamma}^{\text {min }}}^{E_{i}}\left[\frac{T\left(E_{\gamma}\right) \rho\left(E_{i}-E_{\gamma}\right)}{\Delta P_{e x p}\left(E_{i}, E_{\gamma}\right)}\right]^{2}\right. \\
& \left.-2 \sum_{E_{i}=E_{i}^{\min }}^{E_{i}^{\max }} \frac{1}{\sum_{E_{\gamma}^{\prime}=E_{\gamma}^{\min }}^{E_{i}} T\left(E_{\gamma}^{\prime}\right) \rho\left(E_{i}-E_{\gamma}^{\prime}\right)} \sum_{E_{\gamma}=E_{\gamma}^{\min }}^{E_{i}} \frac{T\left(E_{\gamma}\right) \rho\left(E_{i}-E_{\gamma}\right) P_{\exp }\left(E_{i}, E_{\gamma}\right)}{\left[\triangle P_{\exp }\left(E_{i}, E_{\gamma}\right)\right]^{2}}\right\} .
\end{aligned}
$$

One can define

$$
\begin{aligned}
& a\left(E_{i}\right)=\sum_{E_{\gamma}=E_{\gamma}^{\min }}^{E_{i}}\left[\frac{T\left(E_{\gamma}\right) \rho\left(E_{i}-E_{\gamma}\right)}{\triangle P_{\exp }\left(E_{i}, E_{\gamma}\right)}\right]^{2} \\
& b\left(E_{i}\right)=\sum_{E_{\gamma}=E_{\gamma}^{\min }}^{E_{i}} \frac{T\left(E_{\gamma}\right) \rho\left(E_{i}-E_{\gamma}\right) P_{e x p}\left(E_{i}, E_{\gamma}\right)}{\left[\triangle P_{\exp }\left(E_{i}, E_{\gamma}\right)\right]^{2}} \\
& s\left(E_{i}\right)=\sum_{E_{\gamma}=E_{\gamma}^{\min }}^{E_{i}} T\left(E_{\gamma}\right) \rho\left(E_{i}-E_{\gamma}\right) .
\end{aligned}
$$

Inserting the last three equations into Eq. B. 8 gives 


$$
0=\frac{\partial}{\partial T\left(E_{\gamma}\right)} \sum_{E_{i}=E_{i}^{\min }}^{E_{i}^{\max }}\left\{\frac{a\left(E_{i}\right)}{\left[s\left(E_{i}\right)\right]^{2}}-2 \frac{b\left(E_{i}\right)}{s\left(E_{i}\right)}\right\}
$$

or writing out the derivatives,

$$
0=\sum_{E_{i}=E_{i}^{\min }}^{E_{i}^{\max }}\left\{\frac{-2 a\left(E_{i}\right)}{\left[s\left(E_{i}\right)\right]^{3}} \frac{\partial s\left(E_{i}\right)}{\partial T\left(E_{\gamma}\right)}+\frac{1}{\left[s\left(E_{i}\right)\right]^{2}} \frac{\partial a\left(E_{i}\right)}{\partial T\left(E_{\gamma}\right)}+\frac{2 b\left(E_{i}\right)}{\left[s\left(E_{i}\right)\right]^{2}} \frac{\partial s\left(E_{i}\right)}{\partial T\left(E_{\gamma}\right)}-\frac{2}{s\left(E_{i}\right)} \frac{\partial b\left(E_{i}\right)}{\partial T\left(E_{\gamma}\right)}\right\}
$$

The derivatives of the functions $a, b$, and $s$ give

$$
\begin{aligned}
\frac{\partial a\left(E_{i}\right)}{\partial T\left(E_{\gamma}\right)} & =2 T\left(E_{\gamma}\right)\left[\frac{\rho\left(E_{i}-E_{\gamma}\right)}{\triangle P_{\text {exp }}\left(E_{i}, E_{\gamma}\right)}\right]^{2} \Theta\left(E_{i}-E_{\gamma}\right) \Theta\left(E_{\gamma}-E_{\gamma}^{\text {min }}\right) \\
\frac{\partial b\left(E_{i}\right)}{\partial T\left(E_{\gamma}\right)} & =\frac{\rho\left(E_{i}-E_{\gamma}\right) P_{e x p}\left(E_{i}, E_{\gamma}\right)}{\left[\triangle P_{\text {exp }}\left(E_{i}, E_{\gamma}\right)\right]^{2}} \Theta\left(E_{i}-E_{\gamma}\right) \Theta\left(E_{\gamma}-E_{\gamma}^{\text {min }}\right) \\
\frac{\partial s\left(E_{i}\right)}{\partial T\left(E_{\gamma}\right)} & =\rho\left(E_{i}-E_{\gamma}\right) \Theta\left(E_{i}-E_{\gamma}\right) \Theta\left(E_{\gamma}-E_{\gamma}^{\text {min }}\right),
\end{aligned}
$$

where $\Theta$ is the Heaviside function. Inserting the derivatives in Eq. B.11 yields

$$
\begin{aligned}
0= & \Theta\left(E_{\gamma}-E_{\gamma}^{\min }\right) \sum_{E_{i}=E_{i}^{\min }}^{E_{i}^{\max }}\left\{\frac{-2 a\left(E_{i}\right) \rho\left(E_{i}-E_{\gamma}\right)}{\left[s\left(E_{i}\right)\right]^{3}}+\frac{2 T\left(E_{\gamma}\right)}{\left[s\left(E_{i}\right)\right]^{2}}\left[\frac{\rho\left(E_{i}-E_{\gamma}\right)}{\triangle P_{\text {exp }}\left(E_{i}, E_{\gamma}\right)}\right]^{2}\right. \\
& \left.+\frac{2 b\left(E_{i}\right) \rho\left(E_{i}-E_{\gamma}\right)}{\left[s\left(E_{i}\right)\right]^{2}}-\frac{2 \rho\left(E_{i}-E_{\gamma}\right) P_{\text {exp }}\left(E_{i}, E_{\gamma}\right)}{s\left(E_{i}\right)\left[\triangle P_{\text {exp }}\left(E_{i}, E_{\gamma}\right)\right]^{2}}\right\} \Theta\left(E_{i}-E_{\gamma}\right)
\end{aligned}
$$

or 


$$
\begin{aligned}
0= & -2 \sum_{E_{i}=E_{i}^{\min }}^{E_{i}^{\max }} \rho\left(E_{i}-E_{\gamma}\right)\left\{\frac{a\left(E_{i}\right)}{\left[s\left(E_{i}\right)\right]^{3}}-\frac{b\left(E_{i}\right)}{\left[s\left(E_{i}\right)\right]^{2}}+\frac{P_{\text {exp }}\left(E_{i}, E_{\gamma}\right)}{s\left(E_{i}\right)\left[\triangle P_{\exp }\left(E_{i}, E_{\gamma}\right)\right]^{2}}\right\} \Theta\left(E_{i}-E_{\gamma}\right) \\
& +2 T\left(E_{\gamma}\right) \sum_{E_{i}=E_{i}^{\text {min }}}^{E_{i}^{\max }}\left[\rho\left(E_{i}-E_{\gamma}\right)\right]^{2} \frac{1}{\left[s\left(E_{i}\right) \triangle P_{\text {exp }}\left(E_{i}, E_{\gamma}\right)\right]^{2}} \Theta\left(E_{i}-E_{\gamma}\right) .
\end{aligned}
$$

Now we can define

$$
\begin{aligned}
& \varphi\left(E_{i}, E_{\gamma}\right)=\frac{a\left(E_{i}\right)}{\left[s\left(E_{i}\right)\right]^{3}}-\frac{b\left(E_{i}\right)}{\left[s\left(E_{i}\right)\right]^{2}}+\frac{P_{\text {exp }}\left(E_{i}, E_{\gamma}\right)}{s\left(E_{i}\right)\left[\triangle P_{\text {exp }}\left(E_{i}, E_{\gamma}\right)\right]^{2}} \\
& \psi\left(E_{i}, E_{\gamma}\right)=\frac{1}{\left[s\left(E_{i}\right) \triangle P_{e x p}\left(E_{i}, E_{\gamma}\right)\right]^{2}} .
\end{aligned}
$$

Inserting $\varphi$ and $\psi$ in Eq. B.14 gives

$$
T\left(E_{\gamma}\right)=\frac{\sum_{E_{i}=E_{i}^{\min }}^{E_{\max }} \rho\left(E_{i}-E_{\gamma}\right) \varphi\left(E_{i}, E_{\gamma}\right) \Theta\left(E_{i}-E_{\gamma}\right)}{\sum_{E_{i}=E_{i}^{\min }}^{E_{\text {max }}}\left[\rho\left(E_{i}-E_{\gamma}\right)\right]^{2} \psi\left(E_{i}, E_{\gamma}\right) \Theta\left(E_{i}-E_{\gamma}\right)}
$$

The Heaviside function inside the sums requires that $E_{i} \geq E_{\gamma}$; therefore Eq. B.16 can be rewritten as

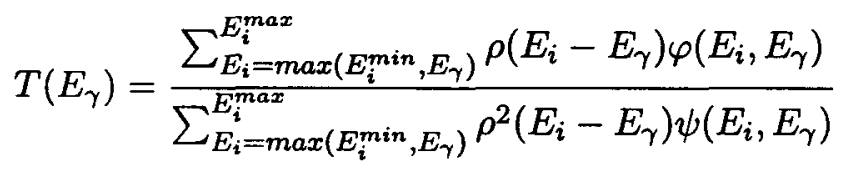

where $\max \left(E_{i}^{\min }, E_{\gamma}\right)$ means use whichever is larger $E_{i}^{\text {min }}$ or $E_{\gamma}$.

Next $\chi^{2}$ as defined in Eq. B.4 is minimized with respect to $\rho\left(E_{i}-E_{\gamma}\right)$ as follows

$$
\frac{\partial}{\partial \rho\left(E_{i}-E_{\gamma}\right)} \chi^{2}=0
$$


or B.10 can be rewritten in terms of $\rho\left(E_{i}-E_{\gamma}\right)$

$$
0=\frac{\partial}{\partial \rho\left(E_{i}-E_{\gamma}\right)} \sum_{E_{i}=E_{i}^{\text {min }}}^{E_{i}^{\text {max }}}\left\{\frac{a\left(E_{i}\right)}{\left[s\left(E_{i}\right)\right]^{2}}-2 \frac{b\left(E_{i}\right)}{s\left(E_{i}\right)}\right\}
$$

For simplicity we call $E_{i}-E_{\gamma}=E_{f}$ in the following equations. Writing out the derivatives explicitly yields

$$
0=\sum_{E_{i}=E_{i}^{\min }}^{E_{i}^{\max }}\left\{\frac{-2 a\left(E_{i}\right)}{\left[s\left(E_{i}\right)\right]^{3}} \frac{\partial s\left(E_{i}\right)}{\partial \rho\left(E_{f}\right)}+\frac{1}{\left[s\left(E_{i}\right)\right]^{2}} \frac{\partial a\left(E_{i}\right)}{\partial \rho\left(E_{f}\right)}+\frac{2 b\left(E_{i}\right)}{\left[s\left(E_{i}\right)\right]^{2}} \frac{\partial s\left(E_{i}\right)}{\partial \rho\left(E_{f}\right)}-\frac{2}{s\left(E_{i}\right)} \frac{\partial b\left(E_{i}\right)}{\partial \rho\left(E_{f}\right)}\right\}
$$

The derivatives of the functions $a, b$, and $s$ with respect to $\rho\left(E_{f}\right)$ give

$$
\begin{aligned}
& \frac{\partial a\left(E_{i}\right)}{\partial \rho\left(E_{f}\right)}=2 \rho\left(E_{f}\right)\left[\frac{T\left(E_{\gamma}\right)}{\Delta P_{e x p}\left(E_{i}, E_{i}-E_{f}\right)}\right]^{2} \Theta\left(E_{i}-E_{f}-E_{\gamma}^{\min }\right) \Theta\left(E_{f}\right) \\
& \frac{\partial b\left(E_{i}\right)}{\partial \rho\left(E_{f}\right)}=\frac{T\left(E_{i}-E_{f}\right) P_{e x p}\left(E_{i}, E_{i}-E_{f}\right)}{\left[\triangle P_{e x p}\left(E_{i}, E_{i}-E_{f}\right)\right]^{2}} \Theta\left(E_{i}-E_{f}-E_{\gamma}^{\min }\right) \Theta\left(E_{f}\right) \\
& \frac{\partial s\left(E_{i}\right)}{\partial \rho\left(E_{f}\right)}=T\left(E_{i}-E_{f}\right) \Theta\left(E_{i}-E_{f}-E_{\gamma}^{\text {min }}\right) \Theta\left(E_{f}\right)
\end{aligned}
$$

Inserting the derivatives in Eq. B.20 yields

$$
\begin{aligned}
0= & \Theta\left(E_{f}\right) \sum_{E_{i}=E_{i}^{\min }}^{E_{i}^{\max }}\left\{\frac{-2 a\left(E_{i}\right) T\left(E_{i}-E_{f}\right)}{\left[s\left(E_{i}\right)\right]^{3}}+\frac{2 \rho\left(E_{f}\right)}{\left[s\left(E_{i}\right)\right]^{2}}\left[\frac{T\left(E_{i}-E_{f}\right)}{\triangle P_{e x p}\left(E_{i}, E_{i}-E_{f}\right)}\right]^{2}\right. \\
& \left.+\frac{2 b\left(E_{i}\right) T\left(E_{i}-E_{f}\right)}{\left[s\left(E_{i}\right)\right]^{2}}-\frac{2 T\left(E_{i}-E_{f}\right) P_{e x p}\left(E_{i}, E_{i}-E_{f}\right)}{s\left(E_{i}\right)\left[\triangle P_{\exp }\left(E_{i}, E_{i}-E_{f}\right)\right]^{2}}\right\} \Theta\left(E_{i}-E_{f}-E_{\gamma}^{\min }\right),
\end{aligned}
$$


or

$$
\begin{aligned}
0= & -2 \sum_{E_{i}=E_{i}^{\min }}^{E_{i}^{\max }} T\left(E_{i}-E_{f}\right)\left\{\frac{a\left(E_{i}\right)}{\left[s\left(E_{i}\right)\right]^{3}}-\frac{b\left(E_{i}\right)}{\left[s\left(E_{i}\right)\right]^{2}}+\frac{P_{\text {exp }}\left(E_{i}, E_{i}-E_{f}\right)}{s\left(E_{i}\right)\left[\triangle P_{e x p}\left(E_{i}, E_{i}-E_{f}\right)\right]^{2}}\right\} \\
& \Theta\left(E_{i}-E_{f}-E_{\gamma}^{\text {min }}\right) \\
& +2 \rho\left(E_{f}\right) \sum_{E_{i}=E_{i}^{\text {min }}}^{E_{i}^{\max }}\left[T\left(E_{i}-E_{f}\right)\right]^{2} \frac{1}{\left[s\left(E_{i}\right) \triangle P_{\text {exp }}\left(E_{i}, E_{i}-E_{f}\right)\right]^{2}} \Theta\left(E_{i}-E_{f}-E_{\gamma}^{\text {min }}\right) .
\end{aligned}
$$

Inserting the definitions for $\varphi$ and $\psi$ in Eq. B.23 gives

$$
\rho\left(E_{f}\right)=\frac{\sum_{E_{i}=E_{i}^{\min }}^{E_{\text {max }}^{\max }} T\left(E_{i}-E_{f}\right) \varphi\left(E_{i}, E_{i}-E_{f}\right) \Theta\left(E_{i}-E_{f}-E_{\gamma}^{\text {min }}\right)}{\sum_{E_{i}=E_{i}^{\min }}^{E_{\text {max }}}\left[T\left(E_{i}-E_{f}\right)\right]^{2} \psi\left(E_{i}, E_{i}-E_{f}\right) \Theta\left(E_{i}-E_{f}-E_{\gamma}^{\min }\right)}
$$

The Heaviside function inside the sums requires that $E_{i} \geq E_{f}+E_{\gamma}^{m i n}$; therefore Eq. B.24 can be rewritten as

$$
\rho\left(E_{f}\right)=\frac{\sum_{E_{i}=\max \left(E_{i}^{\min }, E_{f}+E_{\gamma}^{\min }\right)}^{E_{\max }} T\left(E_{i}-E_{f}\right) \varphi\left(E_{i}, E_{i}-E_{f}\right)}{\sum_{E_{i}=\max \left(E_{i}^{\min }, E_{f}+E_{\gamma}^{\min }\right)}^{E^{\max }} T^{2}\left(E_{i}-E_{f}\right) \psi\left(E_{i}, E_{i}-E_{f}\right)}
$$




$\prod_{\text {APPENDIX }}$

\section{Area Correction for the Primary $\gamma$-ray}

\section{Spectrum}

The primary $\gamma$-ray spectrum $h_{i}$ for an excitation-energy bin $E_{i}$ is obtained from

$$
h_{i}=f_{i}-g_{i}
$$

where $f_{i}$ is the $\gamma$-ray spectrum for the bin $E_{i}$. The spectrum $g_{i}$ is a sum over weighted $\gamma$-ray spectra of all the excitation-energy bins to which the first $\gamma$-ray decays, below the bin $E_{i}$, and is given by

$$
g_{i}=\sum_{j} n_{j}^{i} w_{j}^{i} f_{j}
$$

where $n_{j}^{i}$ accounts for the different cross sections for populating the respective levels, and $w_{j}^{i}$ is the probability of emitting a $\gamma$ ray from the level $E_{i}$ to the level $E_{j}$. The weighting functions are obtained using an iteration procedure described in Chapter 5.

Ideally the area of the primary $\gamma$-ray spectrum should be equal to the area of the observed spectrum minus the area of the spectrum $g_{i}$, as given in Eq. C.1. However, the 
two areas sometimes may not be equal as a result of an improper choice of the weighting function $w$. In that case, we apply an area correction to the spectrum $g_{i}$

$$
A\left(h_{i}\right)=A\left(f_{i}\right)-\alpha A\left(g_{i}\right)
$$

The area $A\left(h_{i}\right)$ can be written as

$$
A\left(h_{i}\right)=A\left(f_{i}\right) / M_{i}
$$

where $M_{i}$ is the $\gamma$-ray multiplicity for the excitation energy $E_{i}$, and can be approximately calculated as

$$
M_{i}=\frac{E_{i}-E_{e}}{\bar{E}_{\gamma}}
$$

where $E_{\mathrm{e}}$ is the excitation energy that the statistical cascade enters along the yrast, and $\bar{E}_{\gamma}$ is the centroid of the average $\gamma$-ray energy in the unfolded $\gamma$-ray spectrum.

By combining Eq. C.3 and C.4 one obtains the correction factor $\alpha$

$$
\alpha=\left(1-\frac{1}{M_{i}}\right) \frac{A\left(f_{i}\right)}{A\left(g_{i}\right)}
$$

which will be used to multiply the subtracted spectrum

$$
h_{i}=f_{i}-\alpha g_{i}
$$




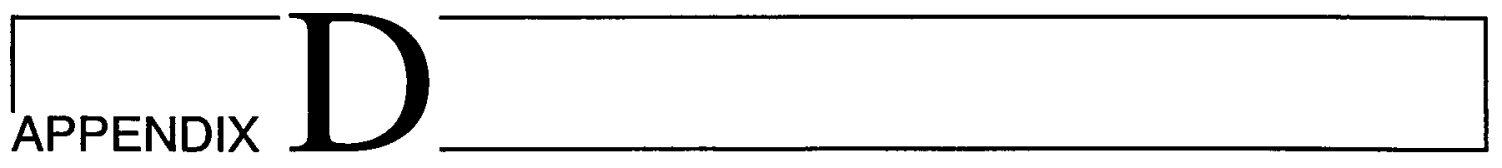

\section{Caloric Curves Derived from Probability}

\section{Density Function}

This appendix is devoted to an idea developed by A. Schiller [82], which may provide insight into the investigation of the thermodynamics of small systems. The examples and arguments given here aid in the interpretation of the experimental results given in Chapter 6 . Thermodynamics deals with large systems, and is very powerful in describing these systems. In a similar manner, thermodynamic quantities have been employed to study small systems. However, in this case one cannot make the same approximations as considered in the thermodynamic limit. Here some misconceptions in the use of thermodynamic quantities for small systems will be addressed.

Caloric curves in thermodynamics are given by

$$
\frac{\partial S}{\partial E}=\frac{1}{T}
$$

in the microcanonical ensemble and by

$$
E=T^{2} \frac{\partial \ln Z}{\partial T}
$$


in the canonical ensemble. These two descriptions give the same result if the system under study is large. However, for small systems such as atomic nuclei, these formulae yield different results. Here, it will be shown that both approaches fail to describe the most probable state of the system. Consider the probability density function $P(E, T)$, which is the probability of a system having energy $E$ for a given temperature $T$ and is given by

$$
P(E, T)=\frac{\Omega(E) \exp (-E / T)}{Z(T)}
$$

$\Omega(E)$ is the multiplicity of states with energy $E$ and $Z(T)$ is the canonical partition function given by

$$
Z(T)=\int_{0}^{\infty} \Omega(E) \exp (-E / T) d E
$$

For convenience, we use the logarithm of $P(E, T)$. The most probable energy of the system at a given $T$ satisfies the condition

$$
\frac{\partial \ln P(E, T)}{\partial E}=0
$$

which, after simple manipulations, yields

$$
\frac{\partial S}{\partial E}=\frac{1}{T}
$$

where $S(E)=\ln \Omega(E)$ is the entropy. This is known as the caloric curve in the microcanonical ensemble. Although we would like to have the most probable value of the system, this expression does not always give the most probable value, but sometimes gives 
the locally least probable value of the system. This is shown below for a simple system. Similarly, the necessary condition for the most probable temperature $T$ for a given $E$ is

$$
\frac{\partial \ln P(E, T)}{\partial T}=0
$$

which yields

$$
E=T^{2} \frac{\partial \ln Z}{\partial T}
$$

This is the caloric curve in the canonical ensemble. Next it will be shown that these two caloric curves give different results for small systems by applying these expressions to a simple system. If we assume the multiplicity of states $\Omega(E)$ is proportional to $E^{n}$, then the caloric curve in the microcanonical ensemble (Eq. D.6) yields

$$
E=n T
$$

However, the canonical ensemble (Eq. D.8) gives a different result

$$
E=(n+1) T
$$

It is clear that using either Eq. D.9 or Eq. D.10 does not make a difference in the thermodynamic limit, i.e., when $n \gg 1$. However, the difference between the two equations can be considerable for small systems. An example of this is shown in Fig. D.1 for the case $n=1$.

The contour lines in Fig. D.1 indicate the probability distribution $P(E, T)$ as a 


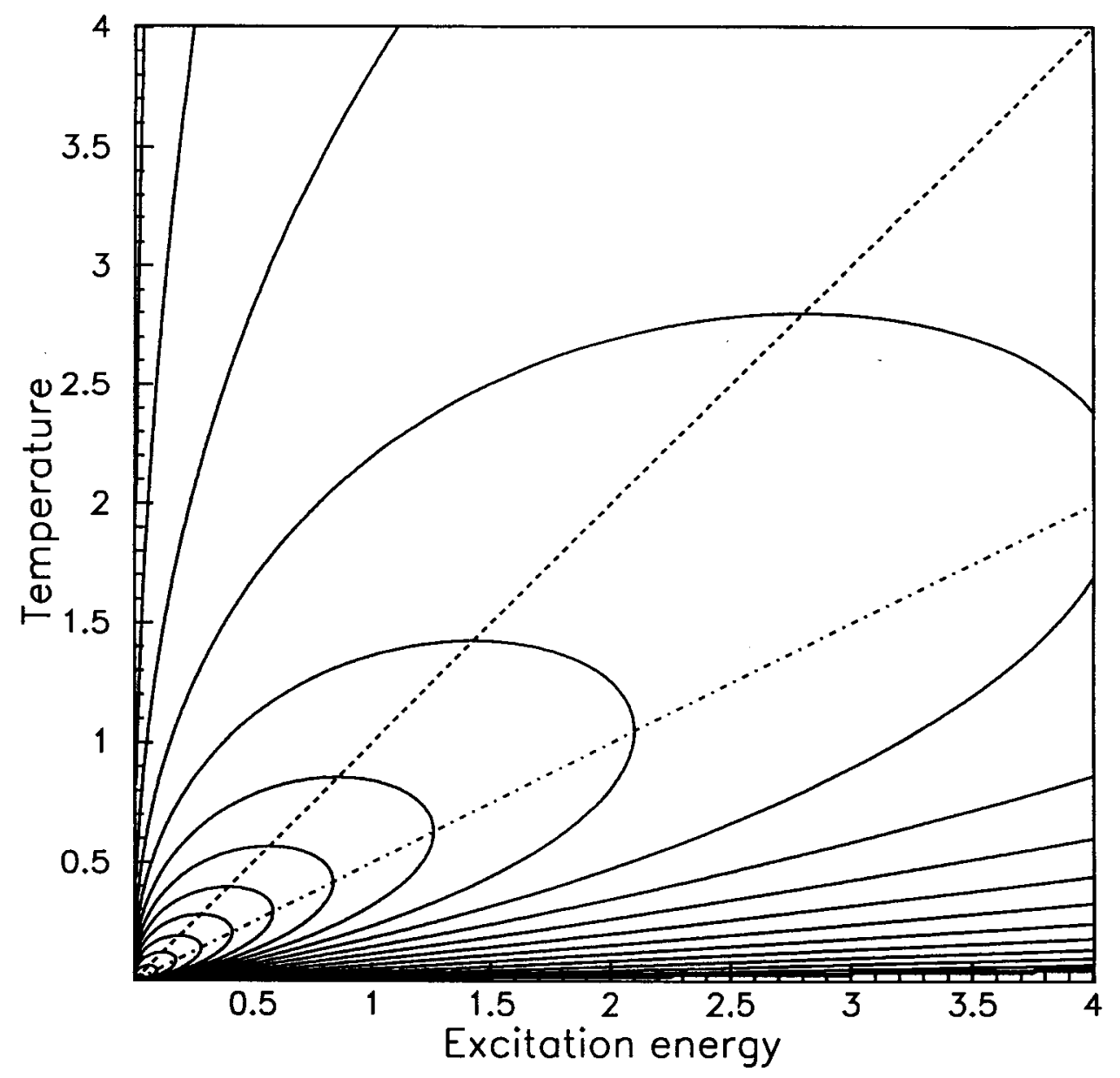

Figure D.1: Probability density function and caloric curves in microcanonical (dashed line) and canonical (dashed-dotted line) ensembles for small systems, in the case of $n=1$.

surface. This can be imagined as the three-dimensional probability distribution $P(E, T)$ projected onto the temperature-energy plane. Thus, each contour line represents equal probability. The caloric curves are shown with dashed and dashed-dotted lines for the microcanonical and canonical ensembles, respectively. The microcanonical caloric curve (MCC) follows the points on the contour lines where the derivative of the distribution $P(E, T)$ with respect to the energy $E$ for a constant temperature is zero, which gives the horizontal tangent (see Eq. D.5). Similarly, the canonical caloric curve (CCC) follows the 


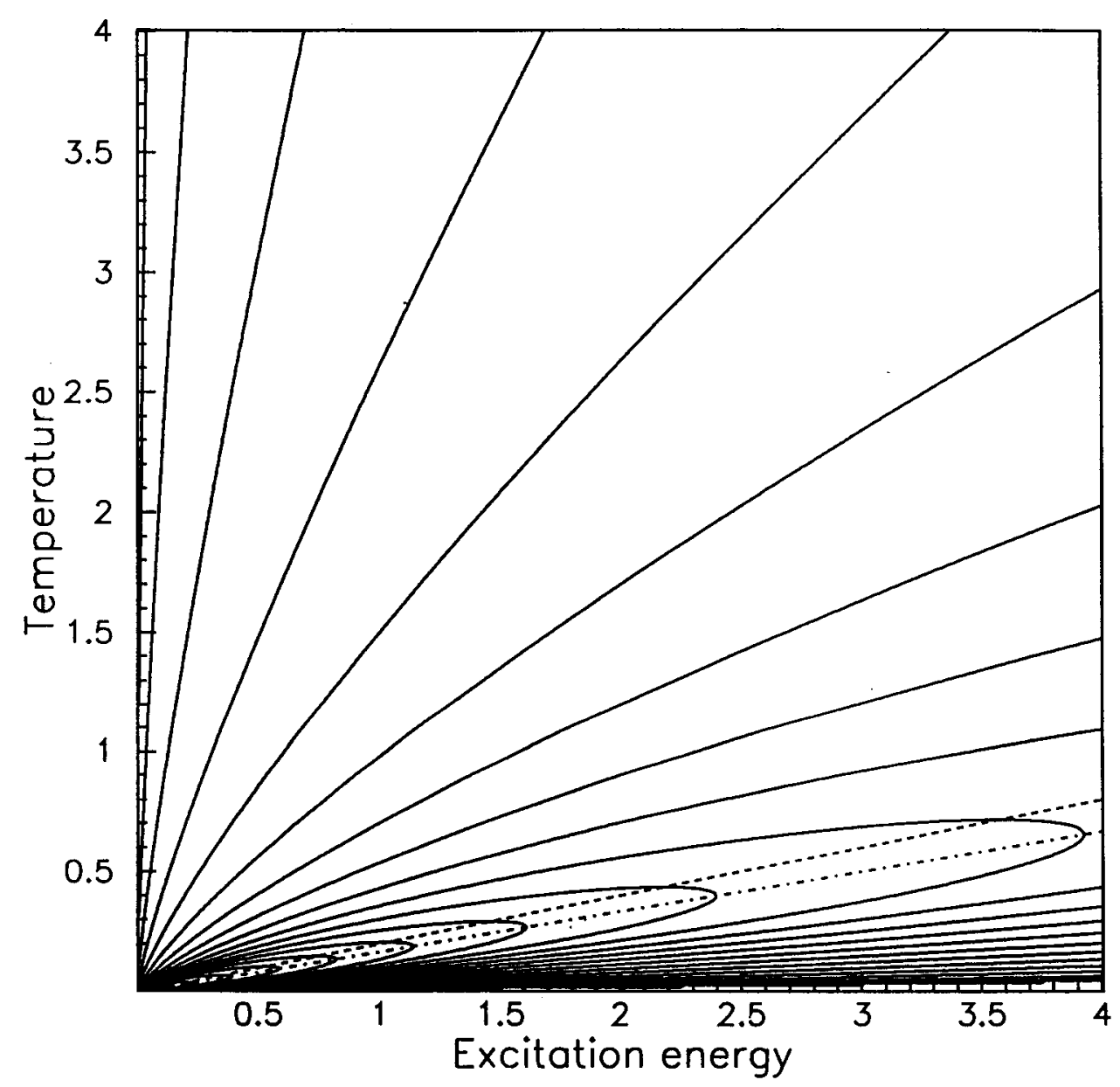

Figure D.2: The same as in Fig. D.1 for the case of $n=5$.

points on the contour lines where the derivative of the distribution for a constant energy is zero, which gives the vertical tangent (see Eq. D.7). It is obvious that the MCC and CCC do not agree in the case of $n=1$, and that the MCC lies above the CCC.

For a larger system with $n=5$, the distribution $P(E, T)$ becomes sharper, and as a result both ensembles give more similar curves, as shown in Fig. D.2. A sharper distribution means that the energy of the system is more certain for a given temperature.

In both of these examples a smooth function for the multiplicity of states is considered. However, this is not the case in nuclei where, for example, step structures due to the breaking of nucleon Cooper pairs are observed in ${ }^{56,57} \mathrm{Fe}$. In the simple model above, 

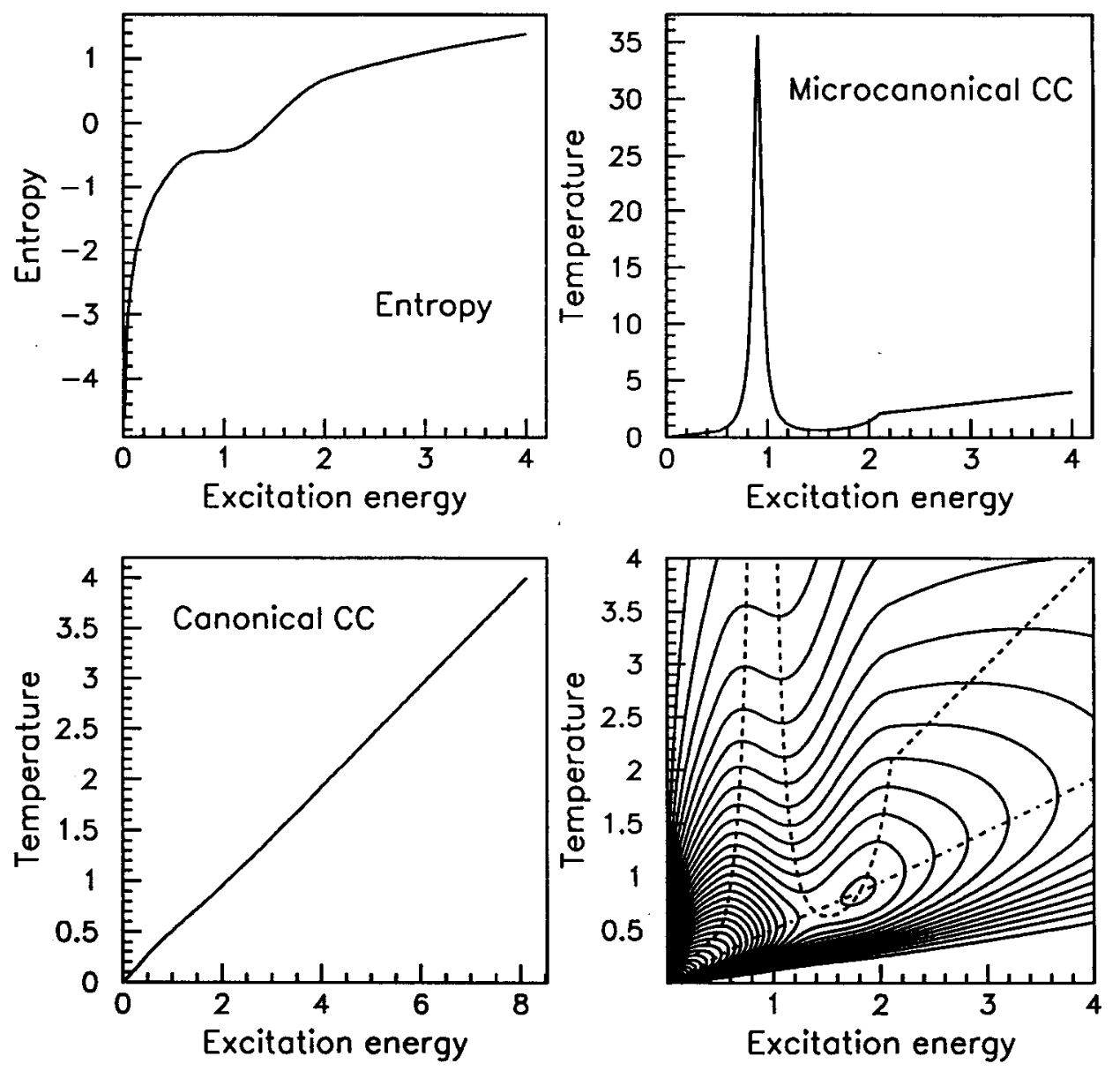

Figure D.3: Effect of entropy fluctuations on the caloric curves.

if a small dip is introduced in the entropy (see the top left panel in Fig. D.3), this will result in large fluctuations in the MCC due to the derivative in Eq. D.6 (the top right panel), while the CCC averages over the structures due to the Laplace transformation of the $\Omega(E)$ in Eq. D.4 (the lower left panel). The probability distribution $P(E, T)$, and the two caloric curves are plotted in the lower right panel in Fig. D.3. The dip introduced in the entropy results in two maxima in the $P(E, T)$. The MCC usually lies above the CCC. The two caloric curves cross each other at two points, the first one is a saddle point and the second one is a local maximum of $P(E, T)$. Notice that the MCC not only follows the most probable values but also the locally least probable values of the system. The CCC 


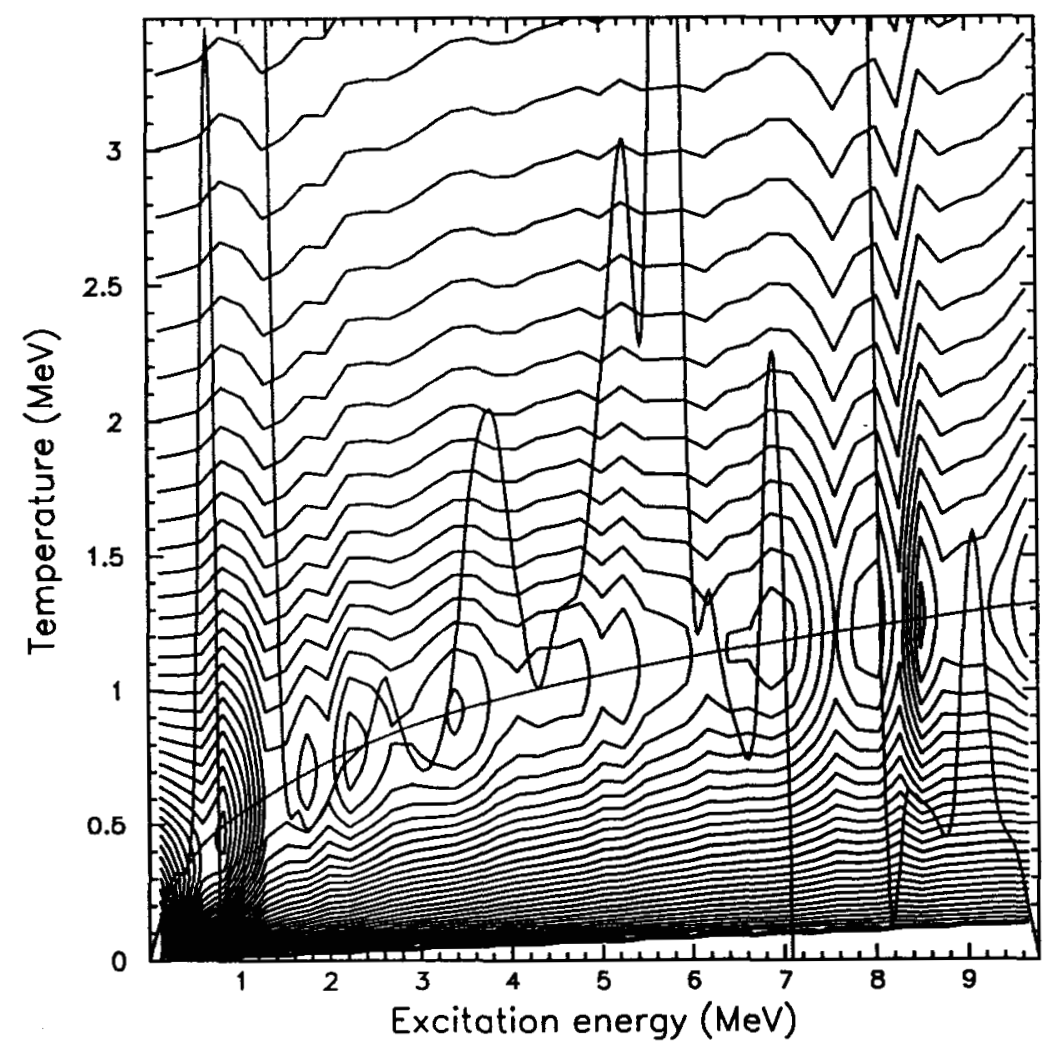

Figure D.4: Probability density function $P(E, T)$ for ${ }^{56} \mathrm{Fe}$.

gives the most probable $T$ for the corresponding $E$. However, the CCC may sometimes describe the system by the least probable values. For example, when the CCC crosses the saddle point (the region where the MCC lies below the CCC (see Fig. D.3)), it describes the system with the least probable $E$ for the corresponding $T$. If there are more dips in the entropy, the distribution $P(E, T)$ exhibits more maxima. Our experimental $P(E, T)$ for ${ }^{56} \mathrm{Fe}$ is an example of such a distribution, as shown in Fig. D.4.

Based on these arguments, it is difficult to construct a caloric curve for a small system. In general caloric curves are constructed by taking the most probable value or the mean value of the distribution of energies (temperatures) for a given temperature (energy). This procedure gives satisfactory results in the thermodynamic limit since the distributions are very sharp. However, for small systems the probability distribution 
$P(E, T)$ may be very broad and may exhibit many maxima. Therefore the traditional methods to construct caloric curves may not be appropriate for small systems. An attempt to describe the system with one specific value of the distribution may result in losing the dynamical information about the system. Therefore, new methods for constructing caloric curves are needed to describe small systems. 


\section{REFERENCES}

[1] P. E. Garrett, H. Lehmann, J. Jolie, C. A. McGrath, M. Yeh, W. Younes, and S. W. Yates, Phys. Rev. C 64, 024316 (2001).

[2] W. Dilg, W. Schantl, H. Vonach, and M. Uhl, Nucl. Phys. A 217, 269 (1973).

[3] J. R. Huizenga, H. K. Vonach, A. A. Katsanos, A. J. Gorski, and C. J. Stephan, Phys. Rev. 182, 1149 (1969).

[4] C. C. Lu, L. C. Vaz, and J. R. Huizenga, Nucl. Phys. A 190, 229 (1972).

[5] H. A. Bethe, Phys. Rev. 50, 332 (1936).

[6] A. Gilbert and A. G. W. Cameron, Can. J. Phys. 43, 1446 (1965).

[7] A. V. Ignatyuk, G. N. Smirenkin, and A. S. Tishin, Yad. Fiz. 21, 485 (1975), [Sov. J. Nucl. Phys. 21, 255 (1975)].

[8] S. K. Kataria, V. S. Ramamurthy, and S. S. Kapoor, Phys. Rev. C 18, 549 (1978).

[9] H. Vonach, M. Uhl, B. Strohmaier, B. W. Smith, E. G. Bilpuch, and G. E. Mitchell, Phys. Rev. C 38, 2541 (1988).

[10] P. Decowski, W. Grochulski, A. Marcinkowski, K. Siwek, and Z. Wilhelmi, Nucl. Phys. A 110, 129 (1968).

[11] A. V. Ignatyuk and Y. N. Shubin, Yad. Fiz. 8, 1135 (1968), [Sov. J. Nucl. Phys. 8, $660(1969)]$.

[12] J. R. Grover, Phys. Rev. 157, 832 (1967).

[13] M. Hillman and J. R. Grover, Phys. Rev. 185, 1303 (1969). 
[14] J. B. French and K. F. Ratcliff, Phys. Rev. C 3, 94 (1971).

[15] R. U. Haq and S. S. M. Wong, Phys. Lett. 93B, 357 (1980).

[16] N. Cerf, Phys. Rev. C 49, 852 (1994).

[17] B. K. Agrawal, T. Sil, J. N. De, and S. K. Samaddar, Phys. Rev. C 62, 044307 $(2000)$.

[18] N. D. Dang, Z. Phys. A335, 253 (1990).

[19] J. L. Egido, L. M. Robledo, and V. Martin, Phys. Rev. Lett. 85, 26 (2000).

[20] A. P. Zuker, Phys. Rev. C 64, 021303(R) (2001).

[21] H. Nakada and Y. Alhassid, Phys. Rev. Lett. 79, 2939 (1997).

[22] H. Nakada and Y. Alhassid, Phys. Lett. B 436, 231 (1998).

[23] S. Liu and Y. Alhassid, Phys. Rev. Lett. 87, 22501 (2001).

[24] V. F. Weisskopf, Phys. Rev. 83, 1073 (1951).

[25] J. M. Blatt and V. F. Weisskopf, Theoretical Nuclear Physics (Wiley, New York, 1952).

[26] B. L. Berman and S. C. Fultz, Rev. Mod. Phys. 47, 713 (1975).

[27] S. S. Dietrich and B. L. Berman, At. Data Nucl. Data Tables 38, 199 (1988).

[28] J. Kopecky, M. Uhl, and R. E. Chrien, Phys. Rev. C 47, 312 (1993).

[29] F. Bečvár, P. Cejnar, R. E. Chrien, and J. Kopecky, Phys. Rev. C 46, 1276 (1992).

[30] F. Bečvár̆, P. Cejnar, J. Honzátko, K. Konečný, I. Tomandl, and R. E. Chrien, Phys. Rev. C 52, 1278 (1995).

[31] S. T. Boneva, V. A. Khitrov, A. M. Sukhovoj, and A. V. Vojnov, Nucl. Phys. A 589, 293 (1995). 
[32] R. E. Chrien, Neutron Radiative Capture (Pergamon Press, New York, 1984).

[33] P. Axel, Phys. Rev. 126, 671 (1962).

[34] D. M. Brink, Ph.D. thesis, Oxford University (1955).

[35] J. Kopecky and M. Uhl, Phys. Rev. C 41, 1941 (1990).

[36] Y. P. Popov, Fiz. Elem. Chastits At. Yadra 13, 1165 (1982), [Sov. J. Part. Nucl. 13, $483(1982)]$.

[37] D. F. Zaretskij and V. K. Sirotkin, Yad. Fiz. 27, 1534 (1978), [Sov. J. Nucl. Phys. 27, 808 (1978)].

[38] S. G. Kadmenskiŭ, V. P. Markushev, and V. I. Furman, Yad. Fiz. 37, 277 (1983), [Sov. J. Nucl. Phys. 37, 165 (1983)].

[39] V. K. Sirotkin, Yad. Fiz. 43, 570 (1986), [Sov. J. Nucl. Phys. 43, 362 (1986)].

[40] J. Kopecky and R. E. Chrien, Nucl. Phys. A 468, 285 (1987).

[41] A. Richter, Prog. Part. Nucl. Phys. 34, 261 (1995).

[42] Handbook for calculations of nuclear reaction data, Tech. Rep. IAEA-TECDOC-1024, IAEA, Vienna (1998).

[43] N. Pietralla, P. von Brentano, R. -D. Herzberg, U. Kneissl, N. Lo Iudice, H. Maser, H. H. Pitz, and A. Zilges, Phys. Rev. C 58, 184 (1998).

[44] J. Enders, H. Kaiser, P. von Neumann-Cosel, C. Rangacharyulu, and A. Richter, Phys. Rev. C 59, R1851 (1999).

[45] D. Bohle, A. Richter, W. Steffen, A. E. L. Dieperink, N. Lo Iudice, F. Palumbo, and O. Scholten, Phys. Lett. B 137, 27 (1984).

[46] M. Igashira, H. Kitazawa, M. Shimizu, H. Komano, and N. Yamamuro, Nucl. Phys. A 457, 301 (1986). 
[47] I. Bergqvist and N. Starfelt, Nucl. Phys. 39, 353 (1962).

[48] G. A. Bartholomew, I. Bergqvist, E. D. Earle, and A. J. Ferguson, Can. J. Phys. 48, 687 (1970).

[49] R. F. Barrett, K. H. Bray, B. J. Allen, and M. J. Kenny, Nucl. Phys. A 278, 204 (1977).

[50] S. Joly, D. M. Drake, and L. Nilsson, Phys. Rev. C 20, 2072 (1979).

[51] E. Melby, M. Guttormsen, J. Rekstad, A. Schiller, S. Siem, and A. Voinov, Phys. Rev. C 63, 044309 (2001).

[52] S. Siem, M. Guttormsen, K. Ingeberg, E. Melby, J. Rekstad, A. Schiller, and A. Voinov, Phys. Rev. C 65, 044318 (2002).

[53] A. Voinov, M. Guttormsen, E. Melby, J. Rekstad, A. Schiller, and S. Siem, Phys. Rev. C 63, 044313 (2001).

[54] A. Schiller, L. Bergholt, M. Guttormsen, E. Melby, J. Rekstad, and S. Siem, Nucl. Instrum. Methods Phys. Res. A 447, 498 (2000).

[55] T. S. Tveter, L. Bergholt, M. Guttormsen, and J. Rekstad, Nucl. Phys. A 581, 220 (1995).

[56] S. Rezazadeh, Master's thesis, University of Oslo (2000).

[57] J. Kantele, Handbook of Nuclear Spectrometry (Academic Press, London, 1995).

[58] A. Schiller, L. Bergholt, M. Guttormsen, E. Melby, S. Messelt, E. A. Olsen, J. Rekstad, S. Rezazadeh, S. Siem, T. S. Tveter, P. H. Vreim, and J. Wikne, Recent Upgrades and Performance of the CACTUS-Detector Array, Tech. Rep. 98-02, Department of Physics, University of Oslo (1998), p. 57.

[59] M. Guttormsen, MAMA Matrix Manipulation Program, Tech. Rep. 94-05, Department of Physics, University of Oslo (1994). 
[60] A. G. Blair and H. E. Wegner, Phys. Rev. 127, 1233 (1962).

[61] F. Ingebretsen, Section for Nuclear Physics and Energy Physics Annual Report 1996, Tech. Rep. 97-08, Department of Physics, University of Oslo (1997), p. 14.

[62] R. Brun, O. Couet, C. Vandoni, and P. Zanarini, PAW - Physics Analysis Workstation, CERN Program Library entry Q121, CERN, Geneva (1989).

[63] M. Guttormsen, T. S. Tveter, L. Bergholt, F. Ingebretsen, and J. Rekstad, Nucl. Instrum. Methods Phys. Res. A 374, 371 (1996).

[64] J. F. Mollenauer, Phys. Rev. 127, 867 (1962).

[65] M. Guttormsen, T. Ramsøy, and J. Rekstad, Nucl. Instrum. Methods Phys. Res. A 255, 518 (1987).

[66] L. Henden, L. Bergholt, M. Guttormsen, J. Rekstad, and T. S. Tveter, Nucl. Phys. A 589, 249 (1995).

[67] T. S. Tveter, M. Guttormsen, J. Rekstad, J. Kownacki, and T. F. Thorsteinsen, Nucl. Phys. A 516, 1 (1990).

[68] M. Guttormsen, L. Bergholt, F. Ingebretsen, G. Løvhøiden, S. Messelt, J. Rekstad, T. S. Tveter, H. Helstrup, and T. F. Thorsteinsen, Nucl. Phys. A 573, 130 (1994).

[69] E. Andersen, H. Helstrup, G. Lovhoiden, T. F. Thorsteinsen, M. Guttormsen, S. Messelt, T. S. Tveter, M. A. Hofstee, J. M. Schippers, and S. Y. Vanderwerf, Nucl. Phys. A 550, 235 (1992).

[70] R. Firestone and V. S. Shirley, Table of Isotopes, vol. 1 (Wiley, New York, 1996).

[71] T. von Egidy, H. H. Schmidt, and A. N. Behkami, Nucl. Phys. A 481, 189 (1988).

[72] A. Bohr and B. R. Mottelson, Nuclear Structure, vol. 1 (World Scientific, Singapore, 1998). 
[73] J. Dobaczewski, P. Magierski, W. Nazarewicz, W. Satuła, and Z. Szymański, Phys. Rev. C 63, 24308 (2001).

[74] T. D. Newton, Can. J. Phys. 34, 804 (1956).

[75] M. Guttormsen, E. Melby, J. Rekstad, A. Schiller, S. Siem, T. Lönnroth, and A. Voinov (2002), nucl-ex/0203013.

[76] A. Schiller et al., (unpublished).

[77] M. Guttormsen, A. Bjerve, M. Hjorth-Jensen, E. Melby, J. Rekstad, A. Schiller, S. Siem, and A. Belić, Phys. Rev. C 62, 024306 (2000).

[78] D. J. Morrissey, W. Benenson, and W. A. Friedman, Annu. Rev. Nucl. Part. Sci. 44, 27 (1994).

[79] C. Kittel and H. Kroemer, Thermal Physics (Freeman, New York, 1980), p.62.

[80] Y. Alhassid, private communication.

[81] A. Schiller, A. Bjerve, M. Guttormsen, M. Hjorth-Jensen, F. Ingebretsen, E. Melby, S. Messelt, J. Rekstad, S. Siem, and S. W. Ødegård, Phys. Rev. C 63, 021306R (2001).

[82] A. Schiller, private communication. 